\title{
Applications of the Gauge/Gravity Duality
}

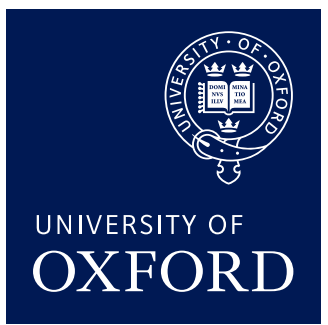

\author{
Jonas Probst \\ St John's College \\ University of Oxford
}

A thesis submitted for the degree of

Doctor of Philosophy

Trinity 2017 


\title{
Applications of the Gauge/Gravity Duality
}

\author{
Jonas Probst \\ St John's College \\ University of Oxford \\ A thesis submitted for the degree of \\ Doctor of Philosophy \\ Trinity 2017
}

\begin{abstract}
This thesis investigates applications of the gauge/gravity duality to strongly coupled quantum field theories. After a review of the duality and of correlators and transport in quantum systems, we present our results on second-order non-conformal hydrodynamics. We derive new Kubo formulae for five second-order transport coefficients in non-conformal relativistic fluids. We then apply these Kubo formulae to a class of non-conformal holographic fluids at infinite coupling. We find strong evidence that the Haack-Yarom identity, known to relate second-order coefficients in conformal holographic fluids at infinite coupling, continues to hold in holographic fluids without conformal symmetry: Within our class of models, we prove that it still holds when leading non-conformal corrections are taken into account, and we show numerically that it is also obeyed beyond leading order. This provides further evidence that the identity may be a universal feature of strongly coupled fluids. Next, we present our results on magnetic spin impurities in strongly correlated systems. We build a holographic two-impurity Kondo model, identifying the inter-impurity interaction as double-trace deformation. Our numerical results for the phase diagram suggest a quantum phase transition between a trivial phase with uncorrelated spins and no Kondo screening, and a non-trivial phase with anti-ferromagnetic correlations and simultaneous Kondo screening. Computing the spectrum in the single-impurity case, we observe Fano resonances, which at low temperatures we identify with the Kondo resonance.
\end{abstract}


Für Muptó 


\section{Acknowledgements}

I would like to express my gratitude to my supervisor Andrei Starinets for his continuous support, reassurance, and inspiring trust throughout my DPhil. I am also very grateful to Andy O'Bannon for his invaluable guidance and advice over the past four years. I would like to thank Ioannis Papadimitriou, Johanna Erdmenger, Carlos Hoyos, Jackson Wu, Christoph Uhlemann, Ronnie Rodgers, and especially Philipp Kleinert for many illuminating discussions and fruitful collaboration. I am thankful to St John's College, the Clarendon Fund, and the European Research Council for the financial support of my doctoral work. Finally, I would like to thank my friends and my family for their support and care, without which this thesis would not have been possible. 


\section{Statement of Originality}

This thesis is based on research and contains no material that has already been accepted, or is concurrently being submitted, for any degree or diploma or certificate or other qualification in this university or elsewhere. To the best of my knowledge and belief this thesis contains no material previously published or written by another person, except where due reference is made in the text.

Chapter 4 is based on P. Kleinert and J. Probst, Second-Order Hydrodynamics and Universality in Non-Conformal Holographic Fluids, JHEP 12 (2016) 091, [1610.01081]. Chapter 5 is based on A. O'Bannon, I. Papadimitriou and J. Probst, A Holographic Two-Impurity Kondo Model, JHEP 01 (2016) 103, [1510.08123], on J. Erdmenger, C. Hoyos, A. O'Bannon, I. Papadimitriou, J. Probst and J. M. S. Wu, Holographic Kondo and Fano Resonances, Phys. Rev. D96 (2017) 021901, [1611.09368], and on J. Erdmenger, C. Hoyos, A. O'Bannon, I. Papadimitriou, J. Probst and J. M. S. Wu, Two-point Functions in a Holographic Kondo Model, JHEP 03 (2017) 039, [1612.02005]. 


\section{Contents}

1 Introduction 1

2 The Gauge/Gravity Duality 5

2.1 String theory and branes . . . . . . . . . . . . . . 5

2.1.1 Open and closed strings ................. 5

2.1.2 Low-energy effective actions . . . . . . . . . . . . . 6

2.1.3 Open/closed string duality . . . . . . . . . . . . . . . 8

2.2 The AdS/CFT correspondence . . . . . . . . . . . . . . . . 9

2.2.1 The near-horizon limit of D3-branes . . . . . . . . . 9

2.2.2 Top-down and bottom-up models . . . . . . . . . . 11

2.2.3 The field/operator correspondence and the GKPW formula . . . 12

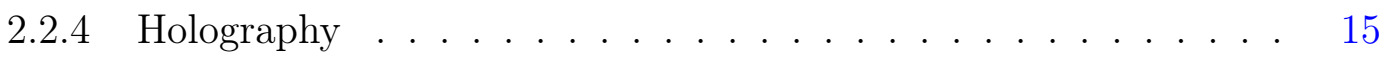

2.2 .5 Thermodynamics ...................... 16

2.2.6 Correlators . . . . . . . . . . . . . . . . 16

2.2.7 Other entries in the holographic dictionary . . . . . . . . . . 17

3 Correlators and Transport 18

3.1 Linear response . . . . . . . . . . . . . . . . . . . . . . 19

3.1.1 Classical example: damped harmonic oscillator . . . . . . . . 19

3.1.2 Time-dependent perturbations in quantum mechanics . . . . . 20

3.1 .3 Kubo formulae . . . . . . . . . . . . . . . . . 22

3.2 Kubo formula for shear viscosity . . . . . . . . . . . . . . . . . . 22

3.2.1 First-order hydrodynamics . . . . . . . . . . . . 22

3.2.2 Metric perturbation .................. 24 
3.2.3 Hydrodynamic transport from holography . . . . . . . . . 25

3.3 Electrical conductivity . . . . . . . . . . . . . . 25

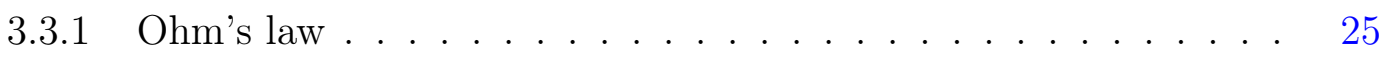

3.3.2 Minimal coupling . . . . . . . . . . . . . . . 25

3.3 .3 London equation . . . . . . . . . . . . . . . . 26

3.3.4 Holographic superconductors . . . . . . . . . . . . . . . . . . 28

3.4 Spectral function and dissipation . . . . . . . . . . . . . . . . . 29

3.4 .1 Classical dissipation . . . . . . . . . . . . . . . . 29

3.4 .2 Quantum dissipation . . . . . . . . . . . . . . 30

3.4.3 Fano resonances . . . . . . . . . . . . . . . . . . 31

4 Second-Order Hydrodynamics and Universality in Non-Conformal Holographic Fluids $\quad 34$

4.1 Introduction and summary . . . . . . . . . . . . . . 34

4.2 New Kubo formulae for non-conformal second-order hydrodynamics . . 38

4.2.1 Sourced fluid stress tensor and Kubo formulae . . . . . . . . . . 38

4.2.2 Holographic calculation . . . . . . . . . . . . . . . . 42

4.3 A class of non-conformal holographic models . . . . . . . . . . . . . 44

4.3.1 Background equations of motion . . . . . . . . . . . 44

4.3.2 Equations for metric perturbations . . . . . . . . . . . . 45

4.4 Solving Einstein's equations . . . . . . . . . . . . . . . 48

4.4.1 Local analysis of background solutions . . . . . . . . . . 49

4.4.2 Solutions for metric perturbations . . . . . . . . . . 50

4.5 Analytical results for second-order transport . . . . . . . . . . . . . 54

4.5.1 Formulae for transport coefficients . . . . . . . . . . . . . . 54

4.5.2 Proof that $H=0$ to leading order away from conformality . . . 57

4.6 Numerical results for second-order transport . . . . . . . . . . . . . 59

4.6.1 Leading non-conformal correction to second-order coefficients . . 59

4.6.2 Two simple families of holographic RG flows . . . . . . . . . . . 60

4.6.3 Second-order coefficients along examples of RG flows . . . . . . 66

4.7 Summary and outlook . . . . . . . . . . . . . . 68 
5 A Holographic Kondo Model $\quad 72$

5.1 Introduction and summary . . . . . . . . . . . . . . . . 72

5.2 The single- and two-impurity Kondo models . . . . . . . . . . . 76

5.2.1 The single-impurity Kondo model . . . . . . . . . . 76

5.2 .2 The two-impurity Kondo model . . . . . . . . . . . . 80

5.3 A holographic two-impurity model . . . . . . . . . . . . . . 85

5.4 Kondo and RKKY couplings from boundary conditions . . . . . . . . 88

5.4.1 Holographic renormalisation . . . . . . . . . . . . . 92

5.4.2 Double-trace Kondo and RKKY couplings . . . . . . . . . . . . 93

5.4 .3 RG transformations . . . . . . . . . . . . . . . . . . . . 96

5.5 The two-impurity phase diagram . . . . . . . . . . . . . 97

5.5.1 Properties of non-trivial solutions . . . . . . . . . . . . 98

5.5.2 Group-theory results . . . . . . . . . . . . . . . . . . . . . 99

5.5 .3 Numerical results . . . . . . . . . . . . . . . . . . . . . 102

5.6 Holographic Kondo and Fano resonances . . . . . . . . . . . . . . . . 110

5.7 Summary and outlook . . . . . . . . . . . . . . 115

6 Conclusion $\quad 119$

$\begin{array}{ll}\text { A Some Properties of Causal Correlators } & 121\end{array}$

B A Class of Non-Conformal Holographic Fluids 123

B.1 Second-order constitutive relations . . . . . . . . . . . . . . . . 123

B.2 Sub-leading modes of metric perturbations . . . . . . . . . . . . . . . 124

B.3 Leading backreaction of the scalar on AdS black branes . . . . . . . . 125

B.4 Numerical construction of RG-flow geometries . . . . . . . . . . . . 129

$\begin{array}{ll}\text { Bibliography } & 134\end{array}$ 


\section{Chapter 1}

\section{Introduction}

Twenty years ago, an intriguing link emerged between two very different kinds of theories. On the one side of this link are quantum gauge theories, akin to the ones describing the electroweak and strong forces which govern nature on the microscopic scales of particle physics. On the other side of this link are theories of gravity, the force which governs nature on the macroscopic scales of cosmology and black holes. At the time, string theory was found to contain extended objects that are described by gauge fields in one limit and by gravity in another limit. Crucially, the limit in which the gravity description becomes appropriate corresponds to the limit in which the gauge theory becomes strongly coupled. The gauge/gravity duality [1-4] asserts that these two descriptions are in fact equivalent for all values of the gauge coupling, albeit typically only one side of the duality is tractable. At weak gauge coupling, the gauge theory is under perturbative control, whereas the dual gravitational degrees of freedom couple to the whole tower of string excitations and can no longer be treated classically. Conversely, at strong gauge coupling, the gauge degrees of freedom defy a perturbative treatment, but they rearrange themselves into a dual description in terms of classical gravity. Notably, the gauge/gravity duality realises the holographic principle $[5,6]$ : it equates a theory of gravity in $d+1$ dimensions with a quantum theory without gravity in $d$ dimensions. More specifically, the best-understood realisations of the duality relate gravity on $(d+1)$-dimensional anti-de Sitter $(\mathrm{AdS})$ spacetime to a conformal field theory $(\mathrm{CFT})$ in $d$ dimensions. Indeed, the gauge/gravity duality, or AdS/CFT correspondence, was originally formulated between the conformal, maximally 
supersymmetric $\mathcal{N}=4$ Yang-Mills theory in 4 dimensions with gauge group $S U(N)$ and type IIB string theory on $A d S_{5} \times S^{5}$, which in the strong-coupling limit of the gauge theory effectively reduces to classical gravity on $A d S_{5}$ [1].

For practical purposes, the most useful feature of the correspondence is that it maps the perturbatively inaccessible strong-coupling regime of a quantum field theory (QFT) to a weakly interacting gravity theory. Conventional calculations in QFTs rely on a perturbative expansion in a small coupling constant. This perturbative approach successfully describes electroweak interactions, asymptotically free quantum chromodynamics (QCD) at high energies, and most condensed matter systems. It fails, however, at strong coupling and is therefore unsuitable for answering many open questions in strongly interacting quantum systems like QCD at low and intermediate energies, including the quark-gluon plasma, or strange metals in condensed matter systems. A powerful alternative is provided by lattice gauge theory, which allows for the numerical evaluation of the quantum path integral also at strong coupling. However, as it requires a Euclidean formulation in which the action provides a valid probability measure, lattice gauge theory is ill-suited for systems at finite density or for computing real-time correlation functions. Gauge/gravity duality by contrast drastically simplifies such calculations at strong coupling. For instance, it reduces the calculation of real-time correlation functions to solving linear wave equations in classical gravity.

These simplifications, however, come at a price: QFTs with a holographic gravity dual are very particular and in fact rather different from realistic theories such as QCD. Firstly, they are typically supersymmetric and conformal. This property becomes less distinctive at finite temperature, though, where both symmetries are broken. Secondly, in order to suppress quantum effects on the dual gravity side one has to take the semiclassical matrix large- $N$ limit of the gauge group, typically $S U(N)$ or similar. Crucially, however, this limit still allows for non-trivial strongly coupled dynamics in the gauge theory. Lastly and most restrictively, holographic QFTs with a tractable gravity dual must have a sparse, gapped operator spectrum at strong coupling. This is necessary to ensure that they can be described by just a few fields in the dual gravity theory. Due to these restrictions, holographic gauge theories with a gravity dual can at best serve 
as useful toy models for strongly coupled real-world systems.

Applications of the gauge/gravity duality have nonetheless been valuable for at least three reasons. One, many low-energy properties of physical systems are insensitive to the details of the UV degrees of freedom. Such universal properties only depend on the IR physics and are thus common to different theories whose renormalisation-group (RG) flows pass close by the same IR fixed point. If we are able to identify universal properties that are shared by a large class of holographic theories, we may hope that these properties are generic features of strong coupling which also apply to realistic, non-holographic theories. The most famous example is the ratio of shear viscosity $\eta$ over entropy density $s$ which, in units of $\hbar / k_{\mathrm{B}}$, takes the value $1 / 4 \pi$ in a large class of holographic systems [7]. This value is vastly different from the one found in weakly coupled theories and, most importantly, is very close to the experimental value observed in the quark-gluon plasma [8]. Chapter 4 of this thesis will investigate a similar, potentially universal relation among second-order hydrodynamic transport coefficients. Two, in many cases there simply are no other tools available that would allow for calculations in strongly interacting systems. Moreover, the dual gravitational perspective offers a geometric language for strongly coupled processes which could not be adequately described with concepts borrowed from weakly interacting quasi-particles [9]. This motivated our work in chapter 5 which, with an eye towards strange metals, investigates magnetic impurities coupled to strongly interacting charge carriers. Three, the fresh perspective offered by the gauge/gravity duality has often inspired results in field theory or gravity that are valid independently of a holographic description. Such results include the discovery of new transport coefficients in hydrodynamics $[10,11]$ and loopholes in no-hair theorems for black holes [12]. Our work on second-order hydrodynamics in chapter 4 led us to the derivation of new Kubo formulae, which are valid for any uncharged relativistic fluid in $(3+1)$ dimensions, irrespectively of whether the fluid admits a holographic gravity dual. Similarly, seeking to build a holographic model with two interacting spin impurities in chapter 5 , we found that the limit of large spin degeneracy suppresses ferromagnetic spin-spin correlations compared to anti-ferromagnetic ones, a result which also holds independently of a holographic description. 
This thesis investigates some applications of the gauge/gravity duality to strongly coupled quantum systems, and is structured as follows. Chapters 2 and 3 review relevant background material. Chapter 2 motivates the origin of the gauge/gravity duality within string theory and summarises the relevant entries in the holographic dictionary, which translates between the gauge and gravity sides. Chapter 3 explains how transport properties and excitation spectra of quantum theories can be extracted from real-time correlation functions. Chapter 4 is based on ref. [13] and contains our work on second-order non-conformal hydrodynamics. We first derive five new Kubo formulae that express second-order transport coefficients in terms of three-point correlators of the stress tensor. We then apply these Kubo formulae to a large class of non-conformal holographic fluids at infinite coupling. We find strong evidence that the Haack-Yarom identity [14], which relates second-order coefficients in conformal holographic fluids at infinite coupling, continues to hold for holographic fluids without conformal symmetry. Within our class of models, we prove that the identity is still obeyed when taking into account leading non-conformal corrections, and show numerically that the identity continues to hold further away from conformal symmetry. Chapter 5 is based on refs. [15-17] and presents our work on a holographic Kondo model. This model [18] describes a magnetic impurity coupled to strongly correlated charge carriers, which are modelled by a holographic CFT. We extend the model by adding a second impurity and including an inter-impurity interaction. We obtain numerical evidence for a quantum phase transition in the two-impurity phase diagram. Computing correlation functions in the holographic single-impurity Kondo model, we observe Fano resonances in the corresponding spectrum and identify the Kondo resonance. We conclude with a summary of our results and suggestions for future research in chapter 6 .

Throughout this thesis we work in units with $c=\hbar=k_{\mathrm{B}}=1$ and use a mostly-plus convention for the metric, denoting the Minkowski metric by $\eta_{\mu \nu}=\operatorname{diag}(-1,1, \ldots, 1)$. We write spacetime points and momenta in flat space as $x^{\mu}=(t, \underline{x})$ and $k^{\mu}=(\omega, \underline{k})$ with $k x \equiv k^{\mu} x^{\nu} \eta_{\mu \nu}=-\omega t+\underline{k} \cdot \underline{x}$. We distinguish a function $f(x)$ from its Fourier transform $f(k)$ only by the argument, $f(k)=\int \mathrm{d} t \mathrm{~d} \underline{x} e^{-i k x} f(x)$. The line element of the $n$-dimensional unit sphere $S^{n}$ is denoted by $\mathrm{d} \Omega_{n}^{2}$. 


\section{Chapter 2}

\section{The Gauge/Gravity Duality}

In this chapter we introduce the gauge/gravity duality, the main topic of this thesis. In section 2.1 we motivate the duality by reviewing its origin in string theory. Section 2.2 describes the main entries in the holographic dictionary which we will use in chapters $4-$ 5 to compute properties of strongly coupled gauge theories from their AdS gravity dual.

\subsection{String theory and branes}

\subsubsection{Open and closed strings}

A propagating string [19-24] swipes out a two-dimensional worldsheet in the ambient $D$-dimensional target space, analogous to the one-dimensional worldline of a point particle. The area of the worldsheet, parametrised by time and space coordinates $\sigma^{\alpha}=(\tau, \sigma)$, is given by the Nambu-Goto action

$$
S=-T \int \mathrm{d}^{2} \sigma \sqrt{-\operatorname{det}\left(\partial_{\alpha} X^{\mu} \partial_{\beta} X^{\nu} \eta_{\mu \nu}\right)}
$$

where $X^{\mu}(\sigma)$ denotes the string's position in the flat target space and $T=1 /\left(2 \pi \alpha^{\prime}\right)$ is the string tension with string length $l_{s}=\sqrt{\alpha^{\prime}}$. Classically, the Nambu-Goto action is equivalent to the Polyakov action

$$
S=-\frac{T}{2} \int \mathrm{d}^{2} \sigma \sqrt{-\gamma} \gamma^{\alpha \beta} \partial_{\alpha} X^{\mu} \partial_{\beta} X^{\nu} \eta_{\mu \nu}
$$

with dynamical worldsheet metric $\gamma_{\alpha \beta}$. The Polyakov action is invariant under diffeomorphisms and under Weyl transformations of $\gamma_{\alpha \beta}$. In other words, the dynamics of a string are governed by a covariant two-dimensional conformal field theory $[21,25]$. 
The redundant two-dimensional metric $\gamma_{\alpha \beta}$ has three independent components. We can fix two of them by a diffeomorphism and the third one by a local Weyl transformation. Conformal symmetry is therefore crucial in ensuring that the Polyakov action contains no more degrees of freedom than the Nambu-Goto action and really describes a string [23].

Strings are either closed, satisfying boundary conditions periodic in $\sigma$, or open. The endpoints of an open string can satisfy Neumann boundary conditions, fixing their derivative $\partial_{\sigma} X^{\mu}=0$, or Dirichlet boundary conditions, fixing their position $X^{\mu}=$ const. If the endpoint of an open string satisfies Dirichlet boundary conditions along $(D-p-1)$ target-space directions, $I=p+1, \ldots, D-1$, then its movement is restricted to a $(p+1)$ dimensional hyperplane called $\mathrm{D}$ (irichlet)-brane or D $p$-brane.

Quantising the Polyakov action gives rise to an infinite tower of states whose mass is set by the inverse string length $1 / l_{s}$. The spectrum of the bosonic string described by (2.2) contains a tachyon with negative mass, indicating the instability of the trivial vacuum. The tachyon disappears if we add fermionic superpartners for the bosonic coordinates $X^{\mu}$ and hereby extend the worldsheet symmetry to a superconformal symmetry. There are five different consistent ways to implement supersymmetry [22]. A common feature of all five is the requirement of a target space with $D=10$ dimensions in order to remove negative-norm states and to maintain global Poincaré and local Weyl symmetry [23]. The bosonic massless excitations of the closed superstring consist of the scalar dilaton $\Phi$, the graviton $G_{\mu \nu}$, and form fields $C_{q}$ (with $q=1,3$ and $q=0,2,4$ for the type IIA and type IIB superstring respectively, see subsection 2.1.2). The massless excitations of an open string whose endpoints lie on a $\mathrm{D} p$-brane contain scalar fields $\phi^{I}$, $I=p+1, \ldots, D-1$, which describe transverse fluctuations of the brane, and a photon $A_{a}, a=0, \ldots, p$. The Dirichlet boundary conditions on a D-brane preserve half of the supercharges of the open superstring [25].

\subsubsection{Low-energy effective actions}

The massless bosonic states of string theory can form large coherent excitations that behave like classical fields, much like a classical electromagnetic field emerges from 
coherent photon excitations. These classical background fields appear as local couplings for a probe string that moves in such a non-trivial background. For instance, a probe string moving on a curved target-space metric $G_{\mu \nu}$, formed by coherent graviton excitations, is described by the natural generalisation of the Polyakov action (2.2):

$$
S=-\frac{T}{2} \int \mathrm{d}^{2} \sigma \sqrt{-\gamma} \gamma^{\alpha \beta} \partial_{\alpha} X^{\mu} \partial_{\beta} X^{\nu} G_{\mu \nu}(x)
$$

The requirement that the theory remain conformal at the quantum level means that the renormalisation process must not introduce a scale dependence for worldsheet couplings like $G_{\mu \nu}$. In other words, their beta functions, which one can compute perturbatively in the string coupling $\alpha^{\prime}$, must vanish. The resulting conditions serve as equations of motion for the classical background fields and, to leading order in $\alpha^{\prime}$, include Einstein's equations for the target-space metric $G_{\mu \nu} \cdot{ }^{1}$ The dilaton $\Phi$ couples to the Euler density of the string worldsheet so that its value at infinity sets the string coupling $g_{s} \equiv$ $\exp \left(\Phi_{X \rightarrow \infty}\right)$, which controls the perturbative expansion in worldsheet topologies, i.e. the loop expansion in the target space.

For the type IIA and IIB superstring, supersymmetry uniquely extends the equations for the bosonic fields to the equations of type IIA and IIB supergravity respectively $[22,24]$. One can consistently set all fields except the dilaton, the graviton, and a single $(q-1)$-form $C_{q-1}$ to zero [4]. The relevant equations of motion in the Einstein frame $g_{\mu \nu} \equiv e^{-\Phi / 2} G_{\mu \nu}$ follow from the target-space action

$$
S=\frac{1}{2 \kappa_{10}^{2}} \int \mathrm{d}^{10} x \sqrt{-g}\left(R-\frac{1}{2}(\partial \Phi)^{2}-\frac{b_{q}}{2} e^{(5-q) \Phi / 2}\left|F_{q}\right|^{2}\right),
$$

where $b_{q}=1 / 2$ if $q=5$ and $b_{q}=1$ otherwise, and $F_{q}=\mathrm{d} C_{q-1}$. The spectra of type IIA and IIB contain $q=2,4$ and $q=1,3,5$ respectively. For $q=5$ we furthermore need to impose the self-duality condition $F_{5}=* F_{5}[22,24]$. One can fix the value of the gravitational coupling $\kappa_{10}$ by equating the tree-level amplitude for closed-string graviton scattering with the corresponding result from the low-energy effective action (in the string frame) [23]. Omitting factors of 2 and $\pi$ (indicated in this section by the

\footnotetext{
${ }^{1}$ Note that all background equations of motion, including Einstein's equations, receive corrections order by order in the string coupling $\alpha^{\prime}$.
} 
symbol $\sim$ ) and denoting the Planck length by $l_{p}$ one finds

$$
\kappa_{10}^{2}=16 \pi l_{p}^{8} \sim g_{s}^{2} l_{s}^{8}
$$

The equations of motion satisfied by the open-string background fields $A_{a}$ and $\phi^{I}$ follow from the Dirac-Born-Infeld (DBI) action [26, 27]

$$
S=-T_{p} \int \mathrm{d}^{p+1} \xi \sqrt{-\operatorname{det}\left(\Gamma_{a b}+2 \pi \alpha^{\prime} F_{a b}\right)}, \quad \Gamma_{a b}=\partial_{a} X^{\mu} \partial_{b} X^{\nu} \eta_{\mu \nu}
$$

on the worldvolume of the $\mathrm{D} p$-brane with induced metric $\Gamma_{a b}$ and $U(1)$ field strength $F=\mathrm{d} A$. One can parametrise the worldvolume by the target-space coordinates parallel to the brane, $\xi^{a}=X^{a}$. The brane's transverse position is then described by the scalar fields, $X^{I}=2 \pi \alpha^{\prime} \phi^{I}[23]$. To leading order in $\alpha^{\prime}$, the DBI action reduces to the Maxwell action plus free scalars $\phi^{I}$. For $N$ coincident $\mathrm{D} p$-branes, there are $N^{2}$ ways to attach the endpoints of an open string with zero length. The gauge fields and scalars thus become $N \times N$ matrices and the $U(1)$ Maxwell action is extended to the $U(N)$ Yang-Mills action, with the gauge field and scalars in the adjoint representation. Supersymmetry extends the Yang-Mills action to the maximally supersymmetric Yang-Mills (SYM) action in $d=p+1$ dimensions [28].

\subsubsection{Open/closed string duality}

Refs. $[29,30]$ realised that $\mathrm{D} p$-branes, described by the open strings ending on them, are in fact dynamical ingredients of type II closed string theory. Consider two parallel $\mathrm{D} p$-branes with an open string stretching between them and let this open string go around in a circle on the branes. By exchanging $\tau$ and $\sigma$ on the worldsheet, we can view this as a closed string propagating from one brane to the other [25,30]. Isolating the leading IR contributions, which come from the graviton and the $C_{p+1}$ boson, reveals that $\mathrm{D} p$-branes have a solitonic tension

$$
T_{p} \sim \frac{1}{g_{s} l_{s}^{p+1}},
$$

and carry a fundamental unit of $C_{p+1}$ charge [30]. Remarkably, the gravitational attraction and the $C_{p+1}$ repulsion between the two D $p$-branes cancels exactly so that the proposed coupling of the branes to the closed-string sector does not compromise the 
branes' stability, as indeed required by supersymmetry. Note that, from relations (2.5) and (2.7), the strength with which $N$ coincident $\mathrm{D} p$-branes couple to the closed-string sector is controlled by [31]

$$
\kappa_{10}^{2} N T_{p} \sim N g_{s}
$$

in units of $l_{s}$. The description of $\mathrm{D} p$-branes in terms of open strings in a flat closedstring background is hence only valid if $N g_{s} \ll 1$.

Type II closed string theory also contains a seemingly very different class of solutions which are charged under $C_{p+1}$, namely black $p$-brane solutions to the relevant part (2.4) of the supergravity action:

$$
\begin{gathered}
g_{\mu \nu} \mathrm{d} x^{\mu} \mathrm{d} x^{\nu}=H(r)^{\frac{p-7}{8}}\left(-f(r) \mathrm{d} t^{2}+\mathrm{d} \underline{x}^{2}\right)+H(r)^{\frac{p+1}{8}}\left(\frac{\mathrm{d} r^{2}}{f(r)}+r^{2} \mathrm{~d} \Omega_{8-p}^{2}\right) \\
e^{\Phi(r)}=H(r)^{\frac{3-p}{4}}, \quad H(r)=1+\left(\frac{L}{r}\right)^{7-p}, \quad f(r)=1-\left(\frac{r_{H}}{r}\right)^{7-p}, \\
F_{p+2}=(p-7) \frac{L^{\frac{7-p}{2}} \sqrt{L^{7-p}+r_{H}^{7-p}}}{H(r)^{2} r^{8-p}}\left(1+\delta_{p, 3} *\right) \mathrm{d} r \wedge \mathrm{d} t \wedge \mathrm{d} x_{1} \wedge \ldots \wedge \mathrm{d} x_{p} .
\end{gathered}
$$

A string moving in this background sees the string-frame metric $G_{\mu \nu}=e^{\Phi / 2} g_{\mu \nu}$ and observes $N$ units of $C_{p+1}$ charge. At zero Hawking temperature, $r_{H}=0$, the $p$-brane solution is called extremal and preserves half of the superstring's supercharges [4]. In terms of fields in the Einstein frame, eq. (2.9), $N$ is in the extremal case given by

$$
N \sim \frac{1}{l_{s}^{7-p}} \int_{S^{8-p}} \frac{1}{g_{s}} e^{(3-p) \Phi / 2} * F_{p+2} \sim\left(\frac{L}{l_{s}}\right)^{7-p} \frac{1}{g_{s}} .
$$

Ref. [30] identified extremal $p$-brane solutions as the appropriate description of $N$ coincident $\mathrm{D} p$-branes in the limit $N g_{s} \gg 1$, in which, according to eq. (2.10), the supergravity approximation holds, $L \gg l_{s}$.

\subsection{The AdS/CFT correspondence}

\subsubsection{The near-horizon limit of D3-branes}

We saw in the previous section that $N$ coincident $\mathrm{D} p$-branes admit two different descriptions in type II string theory. For small $N g_{s} \ll 1$, they appear as hypersurfaces in a flat 
closed-string background with open strings ending on them. For large $N g_{s} \gg 1$, they source the curved $p$-brane solutions of type II closed string theory. Branes with $p=3$ dimensions are special in that they source a constant dilaton field, eq. (2.9), so that the string coupling is constant throughout the 3-brane geometry. In both descriptions we will now take the limit of small energies $E$ in units of $l_{s}[1]$. In the open-string picture, the D3-branes are in this limit effectively described by the maximally supersymmetric $U(N)$ Yang-Mills theory in $d=3+1$ dimensions with $\mathcal{N}=4$ supercharges (see subsection 2.1.2). Moreover, the ambient closed-string background decouples from the branes and reduces to free supergravity in ten-dimensional flat space as, from eq. (2.5), $\kappa_{10} E^{4} \sim g_{s}\left(l_{s} E\right)^{4}[4]$. The Yang-Mills coupling $g_{\mathrm{YM}}$ appears as prefactor $1 / g_{\mathrm{YM}}^{2}$ in the worldvolume action and, from eqs. (2.6) and (2.7), is given by

$$
\frac{1}{g_{\mathrm{YM}}^{2}} \sim T_{3} \alpha^{\prime 2} \sim \frac{1}{g_{s}}
$$

Note that SYM in four dimensions is special in that it is a conformal field theory.

In the closed-string picture, excitations in the asymptotic flat region $r \rightarrow \infty$ also reduce to free supergravity in the low-energy limit. However, a small energy $E$ at $r \rightarrow \infty$ gets blue-shifted to a higher energy $E_{r}=H(r)^{1 / 4} E$ at a finite distance $r$. For small $r$ this becomes, from eq. (2.9), $E_{r} \sim(L / r) E$, so that even in the limit $E l_{s} \rightarrow 0$, arbitrarily high energies $E_{r} l_{s}$ can be observed in the near-horizon region $r / L \rightarrow 0$ with geometry $A d S_{5} \times S^{5}$ :

$$
\mathrm{d} s^{2}=\frac{L^{2}}{r^{2}} \mathrm{~d} r^{2}+\frac{r^{2}}{L^{2}}\left(-\mathrm{d} t^{2}+\mathrm{d} \underline{x}^{2}\right)+L^{2} \mathrm{~d} \Omega_{5}^{2} .
$$

Moreover, low-energy excitations cannot overcome the gravitational barrier between the near-horizon region and the asymptotic flat region so that the two decouple [4]. ${ }^{2}$ We thus have $\mathcal{N}=4 \mathrm{SYM}$ plus free gravity in flat space for $N g_{s} \ll 1$, and all excitations of type IIB string theory on $A d S_{5} \times S^{5}$ plus free gravity in flat space for $N g_{s} \gg 1$. Assuming that the variation in $N g_{s}$ and the low-energy limit commute [31], Maldacena conjectured the equivalence of type IIB string theory on $A d S_{5} \times S^{5}$ and

\footnotetext{
${ }^{2}$ One can compute the potential barrier, as for instance seen by a minimally coupled massless scalar, explicitly by bringing the relevant equation of motion into Schrödinger form, e.g. along the lines of ref. [32] or appendix D in ref. [33].
} 
the superconformal $\mathcal{N}=4 \mathrm{SYM}$ gauge theory for all values of $N g_{s}$ [1]. This is the canonical realisation of the AdS/CFT correspondence or gauge/gravity duality.

Let us now examine the limits in which either side of the correspondence becomes tractable. In order to suppress string interactions, i.e. loops in the target space, we need $g_{s} \rightarrow 0$. Keeping $N g_{s}$ fixed, sending $g_{s} \rightarrow 0$ amounts to letting $N \rightarrow \infty$. On the AdS side, this limit suppresses quantum gravity effects as, from eqs. (2.5) and (2.10), $N \sim\left(L / l_{p}\right)^{1 / 4}$. On the CFT side, this corresponds to the limit of infinite colours. In this limit, only planar Feynman diagrams survive, whose perturbative expansion is controlled by the 't Hooft coupling [4,34,35]

$$
\lambda \equiv N g_{\mathrm{YM}}^{2} \sim N g_{s}
$$

where we used relation (2.11). The strong coupling limit $\lambda \rightarrow \infty$ of the large- $N$ CFT is thus captured by the supergravity limit $N g_{s} \sim\left(L / l_{s}\right)^{1 / 4} \rightarrow \infty$ of classical string theory on AdS. We obtain corrections to the infinite coupling limit by including massive string states in AdS, which are suppressed by powers of $l_{s} / L=\sqrt{\alpha^{\prime}} / L$. In the effective target-space description these are taken into account by $\alpha^{\prime}$ corrections to the supergravity action $[36,37]$.

\subsubsection{Top-down and bottom-up models}

Other brane constructions in string theory or M-theory also lead to explicit realisations of the AdS/CFT correspondence for which both the (closed-string) AdS gravity description and the (open-string) gauge theory description are known (see e.g. [4,38]). These so-called top-down constructions tell us that certain large- $N$ gauge theories at strong coupling are adequately described in terms of classical gravity in AdS spacetimes. One may hope that this relation can be turned upside down, i.e. that a sensible classical gravity theory in AdS describes the strong coupling regime of some large- $N$ gauge theory $[9,38]$. This is the idea behind so-called bottom-up models, simple gravity theories in AdS which only retain the minimum bulk matter content required to model relevant features of a dual field theory (see e.g. [39-42]). The obvious disadvantage of bottom-up models is that we do not know the Lagrangian of the hypothetic field theory dual, if it exists at all. The advantage is that bottom-up models are simpler, 
more versatile, and that they allow us to fully focus on relevant features of the strongly coupled field theory dual.

The remainder of this section describes the main entries in the gauge/gravity dictionary, which translates between the two sides of the duality. Though we will not tie the discussion to specific realisations of the AdS/CFT correspondence, we will often draw on the duality between $A d S_{5} \times S^{5}$ and $\mathcal{N}=4 \mathrm{SYM}$ as a guiding example.

\subsubsection{The field/operator correspondence and the GKPW for- mula}

We now describe how we can put the AdS/CFT correspondence to work and extract properties of the strongly coupled CFT from its AdS dual. In order to relate the two sides, recall the open-string description of D3-branes. The stress tensor of the $\mathcal{N}=4$ CFT on the branes couples to perturbations of the ambient metric around flat space, $\eta_{\mu \nu} \rightarrow g_{\mu \nu}=\eta_{\mu \nu}+\delta g_{\mu \nu}$, through the branes' induced metric $\Gamma_{a b}$, eq. (2.6). In the closed-string picture, metric perturbations in the asymptotic flat region of the 3-brane geometry (2.9) should therefore couple to the stress tensor of $\mathcal{N}=4 \mathrm{SYM}$ at strong coupling. However, the only effect a low-energy gravity wave from the far region has on the decoupled near-horizon region $A d S_{5} \times S^{5}$ is to prescribe the value of the gravity wave at the boundary of $\operatorname{AdS}[2,4] .{ }^{3}$ The realisation that boundary values of fields in AdS couple to (are dual to) operators in the CFT led Gubser, Klebanov, Polyakov [2] and Witten [3] to the GKPW formula:

$$
Z_{\text {gravity }}\left[\phi_{0}(x)\right]=\int_{\phi \rightarrow \phi_{0}} \mathcal{D} \phi e^{-S[\phi]}=Z_{\mathrm{CFT}}\left[\phi_{0}(x)\right]=\left\langle e^{\int \mathrm{d}^{d} x O(x) \phi_{0}(x)}\right\rangle_{\mathrm{CFT}} .
$$

Here, $Z_{\text {gravity }}$ denotes the Euclidean partition function of the quantum gravity theory on AdS, subject to the bulk fields $\phi$ assuming the value $\phi_{0}$ at the AdS boundary. $Z_{\mathrm{CFT}}$ is the Euclidean generating functional for operators $O$ in the CFT, coupled to sources $\phi_{0}$.

\footnotetext{
${ }^{3}$ One can verify this explicitly e.g. for the absorption of gravitons $h_{x y}(r) e^{-i \omega t}$ by 3 -branes [43]. The cross section $\sigma$ is proportional to the s-wave absorption probability $P$ [44], given by the ratio of flux $F_{H}$ ingoing at the horizon to the flux $F_{\text {in }}$ incoming from $r \rightarrow \infty, \sigma=|K|^{2} P$. The only effect of $F_{\text {in }}$ and $|K|^{2}$ is to normalise the wave to 1 at the AdS boundary and to express $\sigma$ in units of the $A d S_{5}$ coupling $\kappa_{5}^{2} \equiv \kappa_{10}^{2} / L^{5} \operatorname{Vol}\left(S^{5}\right), \omega \sigma / 2 \kappa_{10}^{2}=-\left(F_{H} / L^{5}\right) / 2 \kappa_{5}^{2}$. From the optical theorem [4], $\omega \sigma / 2 \kappa_{10}^{2}$ equals the imaginary part of the branes' stress-tensor correlator $[45,46]$, in exact agreement with the AdS/CFT prescription [47].
} 
For this reason, one often says that the field theory lives on the boundary of the AdS bulk. In the classical supergravity limit, the GKPW formula states that the classical gravity on-shell action $S$ is the generating functional for connected correlators in the strongly coupled large- $N$ CFT.

The correspondence between fields and operators implies that large gauge transformations in the AdS bulk act as global symmetry transformations in the CFT [3,4]. In particular, the isometries of $A d S_{5}$ and $S^{5}$ correspond to the conformal symmetry $S O(2,4)$ and the R-symmetry $S U(\mathcal{N}=4) \sim S O(6)$ of $\mathcal{N}=4$ SYM respectively. ${ }^{4}$ Indeed, the Kaluza-Klein (KK) compactification of type IIB supergravity on $S^{5}$ contains the $A d S_{5}$ metric, dual to the CFT stress tensor, and an $S U(4)$ gauge field, dual to the R-current. Together with the other lowest KK modes they form $\mathcal{N}=8$ supergravity on $A d S_{5}[49,50]$, which is believed to be a consistent truncation of the full KK tower [4].

To further illustrate the field/operator correspondence consider a minimally coupled scalar field $\phi$ with mass $m$ and a $U(1)$ gauge field $A$ in $A d S_{d+1}$ with metric

$$
\mathrm{d} s^{2}=g_{m n} \mathrm{~d} x^{m} \mathrm{~d} x^{n}=\frac{L^{2}}{z^{2}}\left(\mathrm{~d} z^{2}+\eta_{\mu \nu} \mathrm{d} x^{\mu} \mathrm{d} x^{\nu}\right) .
$$

The fields $\phi$ and $A$ are dual to a scalar operator $O$ and a global $U(1)$ current $J$ respectively. Near the AdS boundary $z=0$, the local Frobenius solutions [51] to the bulk equations of motion,

$$
\begin{array}{r}
\partial_{m}\left(\sqrt{-g} g^{m n} \partial_{n} \phi\right)-\sqrt{-g} m^{2} \phi=0, \\
\partial_{m}\left(\sqrt{-g} f^{m n}\right)=0, \quad f_{m n}=\partial_{m} A_{n}-\partial_{n} A_{m},
\end{array}
$$

take the form

$$
\begin{aligned}
\phi(x, z) & =\phi_{0}(x) z^{d-\Delta_{+}}+\ldots+v(x) z^{\Delta_{+}}+\ldots \\
A_{\mu}(x, z) & =a_{\mu}(x)+\ldots+j_{\mu}(x) z^{d-2}+\ldots
\end{aligned}
$$

where $\Delta_{+}$is the larger root of $\Delta(\Delta-d)=m^{2} L^{2}$ and we chose a gauge $A_{z}=0$. The solutions to the second-order differential equations are each specified by two modes

\footnotetext{
${ }^{4}$ The isometry group $S O(2, d)$ of $A d S_{d+1}$ is most easily identified by embedding $A d S_{d+1}$ as hyperboloid in $\mathbb{R}^{2, d}[3,4,48]$.
} 
$\phi_{0}(x), v(x)$ and $a_{\mu}(x), j_{\mu}(x)$. When we refer to the boundary values of the fields $\phi$ and $A_{\mu}$ we really mean the leading modes $\phi_{0}$ and $a_{\mu}$.

We shall now determine the (mass) dimension of the CFT operator $O$ and confirm that $a_{\mu}$ has the correct dimension $\left[a_{\mu}\right]=1$ to couple to a conserved current $J^{\mu}$ with dimension $\left[J^{\mu}\right]=d-1$. For this purpose let us see how $\phi_{0}$ and $a_{\mu}$ transform under a scale transformation $x^{\mu} \rightarrow \widetilde{x}^{\mu}=\lambda x^{\mu}$. This scaling is implemented by the bulk diffeomorphism $\left(x^{\mu}, z\right) \rightarrow\left(\lambda x^{\mu}, \lambda z\right)$, which leaves the AdS metric (2.15) invariant. ${ }^{5}$ Under this diffeomorphism the scalar $\phi$ and the vector $A_{\mu}$ transform as

$$
\begin{aligned}
\phi(x, z) \rightarrow \widetilde{\phi}(\lambda x, \lambda z) & =\widetilde{\phi}_{0}(\lambda x)(\lambda z)^{d-\Delta_{+}}+\ldots+\widetilde{v}(\lambda x)(\lambda z)^{\Delta_{+}} \ldots \\
& =\phi(x, z) \\
A_{\mu}(x, z) \rightarrow \widetilde{A}_{\mu}(\lambda x, \lambda z) & =\widetilde{a}_{\mu}(\lambda x)+\ldots+\widetilde{j}_{\mu}(\lambda x)(\lambda z)^{d-2} \ldots \\
& =\frac{\partial x^{m}}{\partial \widetilde{x}^{\mu}} A_{m}(x, z)=\frac{1}{\lambda} A_{\mu}(x, z) .
\end{aligned}
$$

This demonstrates that

$$
\left[\phi_{0}\right]=d-\Delta_{+}, \quad[v]=\Delta_{+}, \quad\left[a_{\mu}\right]=1, \quad\left[j_{\mu}\right]=d-1,
$$

and implies that $\phi_{0}$ couples to an operator $O$ with dimension $d-\left(d-\Delta_{+}\right)=\Delta_{+}$. Moreover, an application of the GKPW formula (2.14) shows that the expectation values $\langle O\rangle$ and $\left\langle J^{\mu}\right\rangle$ correspond to the sub-leading modes $v$ and $j^{\mu}$, as suggested by their mass dimensions [52-54].

Note that the roots of $\Delta(\Delta-d)=m^{2} L^{2}$ are only real if

$$
m^{2} L^{2} \geq-\frac{d^{2}}{4}
$$

This is the Breitenlohner-Freedman (BF) bound, below which scalar fields in AdS have negative energies and become unstable $[38,55,56]$.

\footnotetext{
${ }^{5}$ Note that we are ignoring the gravitational backreaction of $\phi$ and $A$ on the AdS metric. The dual statement is that we are ignoring the fact that sourcing the operators $O$ and $J^{\mu}$ introduces an energy scale and breaks conformal invariance. The backreaction, however, has no effect on one- and two-point functions of $O$ and $J^{\mu}$.
} 


\subsubsection{Holography}

Inspired by the fact that the Bekenstein-Hawking entropy of black holes is proportional to their area rather than to their volume, the authors of refs. $[5,6]$ suggested that any theory of quantum gravity in $d+1$ dimensions should admit a holographic description in terms of a non-gravitational theory in $d$ dimensions. Gauge/gravity duality provides the first explicit realisation of this holographic principle and is thus often simply referred to as holography, while field theories with a gravity dual are called holographic. The extra dimension $z$ on the gravity side of the duality is interpreted as an energy scale in the dual field theory. This interpretation is motivated by the fact that UV divergences in the field theory appear on the gravity side as divergences near the AdS boundary $z \rightarrow 0[3,57-59]$. In order to obtain finite results, we have to renormalise the field theory, which involves the following steps on the gravity side: first we need to regulate the theory by introducing a near-boundary cut-off $z=\epsilon$, then remove all divergences in the gravity action by adding counterterms defined on the cut-off surface, and finally remove the cut-off $[53,54,60-63]$. The GKPW formula (2.14) has to be modified in the sense that functional derivatives of the generating functional must be taken with respect to the fields defined on the cut-off surface. ${ }^{6}$ It is convenient to keep in mind that the derivative of the on-shell action with respect to a field on a constant slice $z=\epsilon$ is equal to the field's canonical momentum with respect to $z$, evaluated on the slice $z=\epsilon[64-67]$.

The interpretation of the radial coordinate $z$ as an energy scale offers a geometric picture of RG flows in the dual field theory [68]. Starting from a CFT in the UV, we can induce an RG flow by sourcing a relevant operator. In the gravity picture, this means that we turn on the boundary value for the corresponding bulk field. Close to the boundary, the geometry remains asymptotically AdS, dual to the UV fixed point. Away from the boundary, however, the field's backreaction on the metric will deform the bulk geometry and break scale invariance. Following ref. [68], ref. [69] combined the holographic description of RG flows with gravity energy conditions to prove that the central charge of holographic theories is monotonically decreasing along RG flows.

\footnotetext{
${ }^{6}$ Note that this means that potential contributions from the interior of the bulk (e.g. from a horizon) are discarded.
} 


\subsubsection{Thermodynamics}

If we work in the classical supergravity limit and compactify Euclidean time on circle of radius $\beta$, the GKPW formula (2.14) tells us that the gravity on-shell action equals $\beta$ times the free energy of the field theory [70]. Finite temperature states of the field theory are thus described by finite temperature solutions in gravity where temperature and entropy are the same on both sides. In particular, field theories in flat space are dual to AdS black-brane geometries with a planar horizon [1]. ${ }^{7}$ We can switch between thermodynamic ensembles by adding finite boundary terms to the gravity action, which implement the desired Legendre transformation in the generating functional [71]. ${ }^{8}$

\subsubsection{Correlators}

In order to find solutions to the second-order bulk equations of motion, we need to impose two boundary conditions. The first boundary condition is to pick the dual source which fixes the leading mode $\phi_{0}$ in the near-boundary expansion. In Euclidean signature (and for static solutions in Lorentzian signature) the second boundary condition is simply regularity at the horizon in the interior of the bulk. For time-dependent fields in Lorentzian signature, however, we do not find irregular and regular solutions at the horizon but incoming and outgoing waves. In order to obtain the causal (retarded) response of the system to the source $\phi_{0}$, we have to impose incoming-wave boundary conditions at the horizon [47,73]: the energy that is dissipated into the system by the external source $\phi_{0}$ (see section 3.4) is dual to the energy absorbed by the black brane. A solution to the boundary value problem (BVP) in AdS will determine the sub-leading mode $v$ in terms of the leading mode $\phi_{0}$, in other words, it will determine the one-point function of the dual operator in the presence of the source $\phi_{0}$. We can compute higher-point functions by taking derivatives of $v$ with respect to $\phi_{0}$. If we are only interested in two-point functions, it is sufficient to solve the BVP for linearised bulk equations of motion. These solutions will capture the correct linear dependence

\footnotetext{
${ }^{7}$ The AdS black-brane metric can e.g. be obtained as the near-horizon limit $r_{H}<r \ll L$ of nearextremal $\left(r_{H} \ll L\right)$ black 3-branes $(2.9)$.

${ }^{8}$ If both near-boundary modes of a bulk field are normalisable, which is the case if the sub-leading mode's mass dimension is above the CFT's unitarity bound, then performing the Legendre transformation does not only change the ensemble but also which operator is quantised in the dual theory [72].
} 
of $v$ on the source, $v \sim-G_{R} \phi_{0}$, where $G_{R}$ denotes the retarded two-point function of the dual operator (see section 3.1). This implies that the quasi-normal modes of the black-brane background, i.e. the complex eigenmodes of plane-wave solutions to the linearised equations of motion which satisfy incoming-wave boundary conditions at the horizon and Dirichlet boundary conditions $\phi_{0}=0$ at the boundary, correspond to poles of $G_{R}$ in momentum-space [47,74].

The computation of correlators was central to early checks of the correspondence between $\mathcal{N}=4 \mathrm{SYM}$ and supergravity on $A d S_{5} \times S^{5}$. Many of these checks were based on comparing anomalies, which are fully determined at one-loop and are thus the same at weak and at strong coupling [4]. Both the conformal symmetry and the R-symmetry of $\mathcal{N}=4 \mathrm{SYM}$ are anomalous when the theory is coupled to external sources. The holographic results for the conformal anomaly [60-63], which shows up in the one-point function of the stress-energy tensor's trace, and for the R-symmetry anomaly, which shows up in the non-conservation of the R-symmetry current $J^{\mu}[3]$ and in three-point functions of $J^{\mu}[75,76]$, agree perfectly with the weak-coupling results in the planar limit.

\subsubsection{Other entries in the holographic dictionary}

Other important entries in the holographic dictionary include Wilson loops and probe strings $[57,77,78]$, flavour degrees of freedom and probe branes [79], and chiral symmetry breaking (see e.g. [38,80-82]). As they are not of immediate relevance to this thesis we will not discuss them further. One item we would like to briefly mention though before concluding this chapter is the holographic description of entanglement entropy (EE) [83]. In holographic field theories, the EE between a sub-region $\mathcal{A}$ and its complement is given by the area of the minimal bulk surface with the same boundary as $\mathcal{A}$, divided by $4 G_{N}[84,85]$. Holographic theories therefore allow for a simple computation of EE and can serve as useful toy models in which we can test supposedly general properties of quantum entanglement. This was the approach we followed in ref. [86], where we studied time-dependent systems far from equilibrium in which a variant of the first law of EE, i.e. the proportionality of changes in energy and EE, was still obeyed. 


\section{Chapter 3}

\section{Correlators and Transport}

The thermal equilibrium of a macroscopic system is fully characterised by a thermodynamic potential, which only depends on a few charges. In order to probe the dynamical properties of a system, we need to perturb it out of static equilibrium, e.g. by stirring it with a spoon, by applying an electric field, or by sending in a gravitational wave. At low energies and for small external perturbations, the macroscopic response of a system will still be characterised by only a few effective parameters known as transport coefficients. As we show in section 3.1, the linear response of a system is described by the retarded Green's functions of its underlying microscopic degrees of freedom. In sections 3.2 and 3.3 we use this relation to express the shear viscosity of a fluid and the electrical conductivity of a material in terms of retarded Green's functions. This serves to illustrate the connection between transport coefficients and retarded Green's functions, which is central to our investigation of second-order hydrodynamics in chapter 4 . In section 3.4 we explain that retarded Green's functions not only capture the transport properties of a system, but contain information on the whole excitation spectrum, and we review Fano resonances, observed in the holographic Kondo model in chapter 5.

The relation between transport properties and retarded correlators largely accounts for the practical power of the gauge/gravity duality, which allows for the straightforward computation of retarded Green's functions in strongly coupled holographic theories as explained in section 2.2. Lattice calculations in strongly coupled theories, by contrast, rely on a formulation in Euclidean time and hence do not give us access to causal real-time correlators such as retarded Green's functions. 


\subsection{Linear response}

\subsubsection{Classical example: damped harmonic oscillator}

Let us introduce some basic concepts using the classical example of a damped harmonic oscillator, coupled to an external driving force $F(t)$ :

$$
\ddot{x}(t)+\gamma \dot{x}(t)+\omega_{0}^{2} x(t)=F(t), \quad \gamma>0
$$

The response of the system to the source $F(t)$ will be captured by the Green's function $G_{R}\left(t, t^{\prime}\right):$

$$
x(t)=-\int \mathrm{d} t^{\prime} G_{R}\left(t, t^{\prime}\right) F\left(t^{\prime}\right) .
$$

We can easily solve eq. (3.1) for the Fourier transform $x(\omega)$ :

$$
x(\omega)=\int \mathrm{d} t e^{i \omega t} x(t)=-G_{R}(\omega) F(\omega)
$$

where

$$
\begin{aligned}
G_{R}(\omega) & =-\frac{1}{-\omega^{2}-i \gamma \omega+\omega_{0}^{2}}=\frac{1}{\left(\omega-\omega_{+}\right)\left(\omega-\omega_{-}\right)} \\
\omega_{ \pm} & \equiv-i \frac{\gamma}{2} \pm \omega_{R}, \quad \omega_{R} \equiv \sqrt{\omega_{0}^{2}-\gamma^{2} / 4}
\end{aligned}
$$

The fact that the response (3.3) is local in frequency space is a consequence of translational invariance and implies that $G_{R}\left(t, t^{\prime}\right)=G_{R}\left(t-t^{\prime}\right)$ only depends on $t-t^{\prime}$. The poles $\omega=\omega_{ \pm}$of $G_{R}(\omega)$ are located at the complex eigenfrequencies of the free linear equation of motion. The fact that all poles lie in the lower half of the complex plane ensures that

$$
G_{R}\left(t-t^{\prime}\right)=\int \frac{\mathrm{d} \omega}{2 \pi} e^{-i \omega\left(t-t^{\prime}\right)} G_{R}(\omega)
$$

vanishes for $t-t^{\prime}<0$ (in which case the contour can be closed in the upper half plane): the response of the system is causal. For this reason, one refers to $G_{R}$ as retarded Green's function.

In the neighbourhood of the poles $\omega=\omega_{ \pm}, G_{R}(\omega)$ is dominated by

$$
G_{R}(\omega) \sim \frac{Z_{ \pm}}{\omega-\omega_{ \pm}}
$$


with real residues $Z_{ \pm} \equiv \pm 1 /\left(2 \omega_{R}\right)$. The real part of $G_{R}(\omega)$ becomes

$$
\operatorname{Re} G_{R}(\omega) \sim Z_{ \pm} \frac{\omega \mp \omega_{R}}{\left(\omega \mp \omega_{R}\right)^{2}+\gamma^{2} / 4}
$$

near the poles, while the imaginary part takes the form of a Lorentzian:

$$
\operatorname{Im} G_{R} \sim-\pi Z_{ \pm} \frac{\gamma / 2}{\pi\left[\left(\omega \mp \omega_{R}\right)^{2}+\gamma^{2} / 4\right]}
$$

In the frictionless limit $\gamma \rightarrow 0^{+}$, the simple poles of the real part move to $\pm \omega_{0}$,

$$
\operatorname{Re} G_{R}(\omega) \sim Z_{ \pm} \frac{1}{\omega \mp \omega_{0}}
$$

while the Lorentzian peaks in the imaginary part narrow down to delta peaks:

$$
\operatorname{Im} G_{R} \sim-\pi Z_{ \pm} \delta\left(\omega \mp \omega_{0}\right)
$$

\subsubsection{Time-dependent perturbations in quantum mechanics}

Formally, the (undamped) harmonic oscillator from the previous subsection can be coupled to the external source $F(t)$ by adding the time-dependent perturbation $\delta H(t)=$ $-x(t) F(t)$ to the Hamiltonian. This is analogous to how quantum systems are coupled to external sources (see e.g. $[71,87]$ ). Consider a quantum system with operators $\left\{O^{i}\right\}$ and Hamiltonian $H_{0}$. We are interested in its response to the perturbation

$$
\delta H_{S}(t)=-\int \mathrm{d} \underline{x} O_{S}^{i}(\underline{x}) \phi_{i}(t, \underline{x})
$$

with external (classical) sources $\phi_{i}(x)$ and all operators in the Schrödinger picture (denoted by subscript $S$ ). The response is most easily found by switching to the interaction picture (denoted by subscript $I$ ),

$$
|\psi(t)\rangle_{I}=U_{0}(t)^{\dagger}|\psi(t)\rangle_{S}, \quad O_{I}(t)=U_{0}(t)^{\dagger} O_{S} U_{0}(t)
$$

where

$$
U_{0}(t)=\mathcal{T} \exp \left(-i \int_{0}^{t} \mathrm{~d} t^{\prime} H_{0}\left(t^{\prime}\right)\right)
$$


is the time-evolution operator of the unperturbed system $H_{0}$, and $\mathcal{T}$ denotes timeordering. The Schrödinger equation shows that states in the interaction picture evolve as

$$
|\psi(t)\rangle_{I}=U\left(t, t_{0}\right)\left|\psi\left(t_{0}\right)\right\rangle_{I}, \quad U\left(t, t_{0}\right)=\mathcal{T} \exp \left(-i \int_{t_{0}}^{t} \mathrm{~d} t^{\prime} \delta H_{I}\left(t^{\prime}\right)\right)
$$

that is, their time-evolution is solely governed by the perturbation $\delta H_{I}$. We will from now on drop the subscript $I$. According to eq. (3.15), an initial mixed state with density matrix $\rho_{0} \equiv \rho(t=-\infty)$ evolves in the interaction picture as

$$
\rho(t)=U(t) \rho_{0} U(t)^{\dagger}, \quad U(t) \equiv U(t,-\infty)
$$

This allows us to compute the response of the expectation value $\left\langle O^{i}(t)\right\rangle$ to the perturbation (3.12):

$$
\begin{aligned}
\left\langle O^{i}(x)\right\rangle & =\operatorname{Tr}\left(\rho O^{i}(x)\right)=\operatorname{Tr}\left(\rho_{0} U(t)^{\dagger} O^{i}(x) U(t)\right) \\
& =\operatorname{Tr}\left(\rho_{0} O^{i}(x)\right)+i \int_{-\infty}^{t} \mathrm{~d} t^{\prime} \operatorname{Tr}\left(\rho_{0}\left[\delta H\left(t^{\prime}\right), O^{i}(x)\right]\right)+\mathcal{O}\left(\phi^{2}\right) \\
& =\left\langle O^{i}(x)\right\rangle_{0}-\int \mathrm{d}^{d} x^{\prime} G_{R}^{i j}\left(x-x^{\prime}\right) \phi_{j}\left(x^{\prime}\right)+\mathcal{O}\left(\phi^{2}\right),
\end{aligned}
$$

where we defined the retarded Green's function or retarded correlator as

$$
G_{R}^{i j}\left(x ; x^{\prime}\right) \equiv-i \theta\left(t-t^{\prime}\right)\left\langle\left[O^{i}(x), O^{j}\left(x^{\prime}\right)\right]\right\rangle_{0}
$$

Crucially, the expectation value in (3.18) is computed in the unperturbed system $H_{0}$. As in the classical case, the response of the system is causal, i.e. $G_{R}^{i j}\left(t, \underline{x} ; t^{\prime}, \underline{x}^{\prime}\right)$ vanishes for $t<t^{\prime}$. If the unperturbed system is translationally invariant, then $G_{R}^{i j}\left(x ; x^{\prime}\right)=$ $G_{R}^{i j}\left(x-x^{\prime}\right)$ only depends on $x-x^{\prime}$ and the linear response is local in momentum space:

$$
\delta\left\langle O^{i}(k)\right\rangle=\int \mathrm{d}^{d} x e^{-i k x} \delta\left\langle O^{i}(x)\right\rangle=-G_{R}^{i j}(k) \phi_{j}(k) .
$$

Causality implies that $G_{R}^{i j}(\omega, \underline{k})$ is analytic in the upper half of the complex $\omega$-plane. Of course, there is nothing preventing us from going beyond linear response and considering quadratic and higher contributions in the perturbative series (3.17), as we will in fact do in chapter 4 . 


\subsubsection{Kubo formulae}

In order to complete the analogy with the classical example, we still need to show that the eigenmodes of a quantum system, too, show up as poles of $G_{R}(k)$. To do so, we first need to identify which equations of motion these eigenmodes should correspond to. Because we are ultimately interested in observable low-energy phenomena, we will consider the (classical) equations of motion that are provided by the appropriate effective or phenomenological low-energy description. Such a description will depend on a couple of parameters known as transport coefficients, which ideally can be measured in the laboratory. A simple example to keep in mind is the diffusion of a charge density fluctuation $\rho(x) \equiv \delta\langle n(x)\rangle$, effectively described by the equation $\partial_{t} \rho-D \underline{\nabla}^{2} \rho=0$. It depends on a single transport coefficient, the diffusion constant $D$. The idea is to compute the linear response of the one-point function to an external source within the effective theory, and equate the result with the general expression (3.17). On the one hand, this allows us to derive so-called Kubo formulae which express transport coefficients such as $D$ in terms of retarded Green's functions at low energies $[88,89]$. On the other hand, it serves to demonstrate that the eigenmodes of the effective equations appear as poles of $G_{R}(k)$. Indeed, if the effective equations explicitly contain the operator source, we can solve them directly with a Green's function, just as we did in the case of the classical oscillator, and any eigenmode will automatically appear as a pole of $G_{R}(k)$. This will be the case in sections 3.2 and 3.3. Yet, even if the source does not explicitly show up in the effective equation, as in the case of the diffusion example, it is possible to show that the eigenmodes appear as poles of $G_{R}$ by adiabatically preparing an initial charge fluctuation and letting it evolve freely afterwards $[87,90]$.

\subsection{Kubo formula for shear viscosity}

\subsubsection{First-order hydrodynamics}

Hydrodynamics [90,91], the subject of chapter 4, is the effective theory for low-energy fluctuations around thermal equilibrium. The equilibrium state of a fluid is fully characterised by the expectation values of global charge densities such as energy and mo- 
mentum density, or equivalently by their conjugate variables such as temperature $T$ and fluid velocity $u^{\mu}, u^{\mu} u_{\mu}=-1$ [92]. Hydrodynamics assumes that slowly-varying fluctuations around thermal equilibrium are still fully governed by the conservation equations for charge densities: the relevant degrees of freedom are local charge densities (or their conjugates), representing the expectation values of charge densities in patches of local equilibrium. These fluid patches are small compared to macroscopic scales like temperature, but large compared to all microscopic interaction scales. An uncharged relativistic fluid on a four-dimensional background with metric $g_{(0) \mu \nu}$ can thus be described in terms of a local temperature field $T(x)$ and a local fluid-velocity field $u^{\mu}(x)$. Its dynamics are governed by the conservation of stress-energy:

$$
\nabla_{\mu}\left\langle T^{\mu \nu}(x)\right\rangle=0
$$

Constitutive relations, which express $\left\langle T^{\mu \nu}(x)\right\rangle$ in terms of the fluid variables $T(x)$ and $u^{\mu}(x)$, are constructed as an effective low-energy expansion in derivatives of the fluid variables [90]. To zeroth order $\mathcal{O}\left(\partial^{0}\right)$, one neglects interactions between patches of local equilibrium and $\left\langle T^{\mu \nu}(x)\right\rangle$ can be parametrised as a perfect fluid,

$$
\left\langle T^{\mu \nu}(x)\right\rangle=\epsilon(x) u^{\mu}(x) u^{\nu}(x)+p(x)\left[u^{\mu}(x) u^{\nu}(x)+g_{(0)}^{\mu \nu}(x)\right]+\mathcal{O}(\partial),
$$

with local energy density $\epsilon(x)$ and local pressure $p(x)$, related by the equilibrium equation of state. Dissipation between local equilibrium patches is incorporated by adding a symmetric tensor $\Pi^{\mu \nu}$ that depends on derivatives of the fluid variables and the metric. We may shift the fluid variables by derivatives as long as $\left\langle T^{\mu \nu}(x)\right\rangle$ remains unchanged. We can use this gauge-like freedom to ensure that $u_{\mu}(x)\left\langle T^{\mu \nu}(x)\right\rangle=\epsilon(x) u^{\nu}(x)$ is obeyed to all orders in the gradient expansion, i.e. $u_{\mu} \Pi^{\mu \nu}=0$ (Landau frame) [90,93]. To construct $\Pi^{\mu \nu}$ to first order in derivatives, it is convenient to go to a local rest frame at $x$, where $u^{\mu}(x)=\left(u^{0}(x), \underline{v}(x)\right)=(1, \underline{0})$ and hence $\Pi_{0 \nu}(x)=0$ at $x$ [94]. Translational invariance (i.e. $\Pi_{i j}=0$ if $\underline{v}$ is constant) and rotational invariance (i.e. $\Pi_{i j}=0$ for uniform spatial rotations $\underline{v}=\underline{\Omega} \times \underline{x}$ ) require that [91]

$$
\Pi_{i j}=-\eta\left(\partial_{i} u_{j}+\partial_{j} u_{i}-\frac{2}{3} \delta_{i j} \partial_{k} u^{k}\right)-\zeta \delta_{i j} \partial_{k} u^{k}
$$


on a flat background $g_{(0) \mu \nu}=\eta_{\mu \nu}$. The transport coefficients $\eta$ and $\zeta$ are the shear and bulk viscosity respectively. Generalising to a curved background $g_{(0) \mu \nu}$ and going back to an arbitrary frame by introducing the projector $\Delta^{\mu \nu}(x) \equiv u^{\mu} u^{\nu}+g_{(0)}^{\mu \nu}$, the stress-tensor takes the form [94]

$$
\begin{aligned}
\left\langle T^{\mu \nu}(x)\right\rangle & =\epsilon(x) u^{\mu}(x) u^{\nu}(x)+p(x)\left[u^{\mu}(x) u^{\nu}(x)+g_{(0)}^{\mu \nu}(x)\right]+\Pi^{\mu \nu}(x)+\mathcal{O}\left(\partial^{2}\right), \\
\Pi^{\mu \nu} & =-\eta \Delta^{\mu \rho}\left(\nabla_{\rho} u_{\sigma}+\nabla_{\sigma} u_{\rho}-\frac{2}{3} g_{(0) \rho \sigma} \nabla_{\lambda} u^{\lambda}\right) \Delta^{\sigma \nu}-\zeta \Delta^{\mu \nu} \nabla_{\lambda} u^{\lambda} .
\end{aligned}
$$

\subsubsection{Metric perturbation}

To illustrate how linear response theory, introduced in section 3.1, can be used to compute transport coefficients, we shall derive the Kubo formula for the shear viscosity $\eta$. The source of the stress tensor, namely the external background metric, explicitly enters the hydro equations of motion (3.20) through the covariant derivative. Unlike in the example of the diffusion equation we can therefore compute the response of $\left\langle T^{\mu \nu}\right\rangle$ to an external source directly from the equations of motion. Starting from a fluid in equilibrium in flat space, it is convenient to only turn on the transverse-tensor perturbation $h_{x y}(t, z)$, as such a perturbation couples neither to longitudinal sound fluctuations nor to transverse shear fluctuations of the fluid variables: to linear order in $h_{x y}$, the equations of motion reduce to the unsourced equations $\partial_{\mu}\left\langle T^{\mu \nu}(x)\right\rangle=0$ and the fluid remains at rest [94]. Plugging the perturbed metric and the trivial solution for the fluid variables into the constitutive relation (3.23), we find that the response of the transverse-tensor component, with equilibrium pressure $p$, is given by

$$
\left\langle T^{x y}(x)\right\rangle=\left(-p-\eta \partial_{t}\right) h_{x y}(t, z)
$$

Comparing this with the linear response result, eq. (3.17), we can read off the lowmomentum expansion of the retarded correlator between $T^{x y}$ and $T^{x y}$,

$$
G_{R}^{x y, x y}(\omega, 0,0, q)=p-i \omega \eta+\mathcal{O}\left(\omega^{2}, q^{2}\right)
$$

from which the Kubo formula for shear viscosity follows:

$$
\eta=-\lim _{\omega \rightarrow 0} \frac{1}{\omega} \operatorname{Im} G_{R}^{x y, x y}(\omega, \underline{k}=\underline{0}) .
$$




\subsubsection{Hydrodynamic transport from holography}

Kubo formulae like (3.26) allow us to compute measurable transport coefficients such as $\eta$ from quantum correlators. In particular, we can compute the transport coefficients in theories with a holographic gravity dual by the methods described in section 2.2. Refs. $[43,95,96]$ were the first to study hydrodynamic transport in holographic theories, see ref. [94] for an early review. Chapter 4 will discuss the hydrodynamic properties of holographic theories in greater detail.

\subsection{Electrical conductivity}

\subsubsection{Ohm's law}

Ohm's law can be viewed as another straightforward application of linear response theory. Consider a quantum system with a conserved $U(1)$ current $J^{\mu}(x)$, sourced by an external $U(1)$ gauge field $A_{\mu}(x)$. In a static gauge $A_{t}=0$, turning on the source $A_{i}(t)$ is equivalent to turning on a time-dependent electric field $E_{i}(t)$ :

$$
E^{i}(t)=F^{t i}(t)=-\partial_{t} A_{i}(t) \quad \Longrightarrow \quad E_{i}(\omega)=i \omega A_{i}(\omega)
$$

Plugging this into the linear response result (3.17) yields

$$
\left\langle J^{i}(\omega)\right\rangle=\left\langle J^{i}(\omega)\right\rangle_{0}-\frac{G_{R}^{J^{i} J^{j}}}{i \omega} E_{j}(\omega) .
$$

For this expression to take the form of Ohm's law, $\left\langle J^{i}\right\rangle=\sigma^{i j} E_{j}$, we need to show that the expectation value of the current in the unperturbed state, $\left\langle J^{i}\right\rangle_{0}$, is indeed linear in $E_{j}$

\subsubsection{Minimal coupling}

The current density operator $J^{i}$ is itself linear in $A_{i}$ because the charge carriers are minimally coupled to the gauge field $A_{i}$. To illustrate this statement consider a complex scalar field $\phi$ in flat space with action

$$
S=-\int \mathrm{d}^{d} x\left(\left(D^{\mu} \phi\right)^{\dagger}\left(D_{\mu} \phi\right)+m^{2} \phi^{\dagger} \phi\right)
$$


minimally coupled to $A_{\mu}$ via the covariant derivative $D_{\mu}=\partial_{\mu}-i e A_{\mu}$. Because the action contains a term quadratic in $A_{\mu}$, the conserved electric current

$$
J_{\mu}=-i e\left(\phi^{\dagger} \partial_{\mu} \phi-\left(\partial_{\mu} \phi\right)^{\dagger} \phi\right)-2 e^{2} \phi^{\dagger} \phi A_{\mu}
$$

contains a term linear in $A_{\mu}$. Note that the Hamiltonian, unlike the Lagrangian, contains a perturbation of the form (3.12):

$$
H=\int \mathrm{d} \underline{x}\left(-\left(D^{\mu} \phi\right)^{\dagger}\left(D_{\mu} \phi\right)+m^{2} \phi^{\dagger} \phi\right)+\delta H, \quad \delta H=-\int \mathrm{d} \underline{x} J^{\mu} A_{\mu} .
$$

In the non-relativistic limit,

$$
\phi=\frac{e^{-i m t}}{\sqrt{2 m}} \psi, \quad\left|i \partial_{t} \psi\right| \ll m|\psi|,
$$

in which the minimally coupled Klein-Gordon equation reduces to the minimally coupled Schrödinger equation, the current (3.30) becomes

$$
J^{0}=e \psi^{\dagger} \psi, \quad J^{i}=-\frac{i e}{2 m}\left(\psi^{\dagger} \partial^{i} \psi-\left(\partial^{i} \psi\right)^{\dagger} \psi\right)-\frac{e^{2}}{m} \psi^{\dagger} \psi A^{i} .
$$

In a static equilibrium state we thus find

$$
\left\langle J^{i}\right\rangle_{0}=-e \rho A^{i}
$$

where the charge density $\rho$ is given by $\rho=2 e\left\langle\phi^{\dagger} \phi\right\rangle_{0}$ in the relativistic and $\rho=$ $e\left\langle\psi^{\dagger} \psi\right\rangle_{0} / m$ in the non-relativistic case. While eq. (3.34) holds more generally, the explicit expressions for $\rho$ rely on weakly coupled quasiparticles $[97,98]$. Combined with eq. (3.28) and with $E_{i}=i \omega A_{i}$, eq. (3.34) yields Ohm's law, together with the Kubo formula for the optical or alternating current $(A C)$ conductivity $\sigma^{i j}(\omega)[97,98]$ :

$$
\sigma^{i j}(\omega)=-\frac{1}{i \omega}\left(e \rho \delta^{i j}+G_{R}^{J^{i} J^{j}}(\omega, \underline{0})\right) .
$$

\subsubsection{London equation}

In most systems, the pole in the imaginary part of $\sigma(\omega)$, eq. (3.35), is cancelled by terms in the retarded current-current correlator [98]. If, however, a singular contribution of the form

$$
\sigma(\omega) \sim-\frac{\nu_{s}}{i \omega}
$$


persists, in other words, if

$$
\underline{J}(\omega) \equiv\langle\underline{J}(\omega)\rangle \sim-\nu_{s} \underline{A}(\omega)
$$

then this has two important consequences. First, the pole in the imaginary part of $\sigma(\omega)$ requires, via the Kramers-Kronig relations, that the real part contains a delta peak, $\operatorname{Re} \sigma(\omega) \sim \nu_{s} \pi \delta(\omega)$ : The direct current $(D C)$ conductivity $\sigma(\omega \rightarrow 0)$ is therefore infinite. This happens both in normal conductors without momentum dissipation and in superconductors [99]. Eq. (3.37) is known as London equation and provides a phenomenological description of superconductivity [100,101].

Second, if the source $A_{i}$ is itself dynamical and satisfies Maxwell's equations, as it does in the case of a superconductor, then eq. (3.37) implies that the material expels magnetic fields $\underline{B}$ which effectively acquire a mass [101]:

$$
\nu_{s} \underline{B}=\nu_{s} \underline{\nabla} \times \underline{A}=-\underline{\nabla} \times \underline{J}=-\underline{\nabla} \times\left(\underline{\nabla} \times \underline{B}-\partial_{t} \underline{E}\right)=\left(\underline{\nabla}^{2}-\partial_{t}^{2}\right) \underline{B} .
$$

How the absence of momentum dissipation leads, independently of superconductivity, to a singular conductivity of the form (3.36) can be illustrated in the Drude model [102]. In this model, conduction electrons move at an average velocity $\underline{v}$ which satisfies the phenomenological equation of motion

$$
m \frac{\mathrm{d} \underline{v}}{\mathrm{~d} t}=-m \frac{v}{\tau}+e \underline{E},
$$

where the relaxation time $\tau$ is the average time between collisions. Eq. (3.39) is solved by

$$
\underline{v}(\omega)=\frac{e}{m}\left(\frac{\tau}{1-i \omega \tau}\right) \underline{E}(\omega)
$$

resulting in a current density $\underline{J}(\omega)=e n \underline{v}(\omega)$ and AC conductivity given by

$$
\sigma=\nu \frac{\tau}{1-i \omega \tau}, \quad \nu \equiv \frac{e^{2} n}{m},
$$

where $n$ denotes the density of the conduction electrons. In the limit of vanishing dissipation, $\tau \rightarrow \infty$, the imaginary part of the Drude conductivity acquires a pole of the form (3.36) and the Lorentzian in the real part narrows down to a delta peak:

$$
\operatorname{Im} \sigma(\omega) \stackrel{\tau \rightarrow \infty}{\longrightarrow} \frac{\nu}{\omega}, \quad \operatorname{Re} \sigma(\omega) \stackrel{\tau \rightarrow \infty}{\longrightarrow} \nu \pi \delta(\omega)
$$




\subsubsection{Holographic superconductors}

One of the main motivations to study condensed matter systems using holographic methods is the desire to understand the strongly coupled critical theory which is believed to govern the strange metal phase in high- $T_{c}$ superconductors [9,71, 103-105]. Building on the results from [12], the authors of refs. $[106,107]$ showed that it is indeed possible to realise superconductivity in strongly coupled theories with a gravity dual.

Superconductivity is characterised by the spontaneous breaking of the electromagnetic $U(1)$ symmetry: Below a critical temperature, a charged operator $O$ condenses and the photon $A_{\mu}$ effectively acquires a mass term $\sim \nu A^{\mu} A_{\mu}$. This produces a diamagnetic current of the London form (3.37) [71]. We therefore expect the minimal bulk ingredients for a holographic superconductor to be Einstein-Maxwell gravity coupled to a charged field $\psi$ dual to $O$ [71]. In order to distinguish high and low temperatures $T$, we also need at least one more energy scale to which $T$ can be compared. The simplest way is to source a charge density $J^{t}$ by turning on a chemical potential $\mu$, given by the boundary value of the bulk Maxwell field $A_{t}$. Finally, the microscopic bulk dynamics must be such that $\psi$ vanishes at high $T$ while a non-trivial profile for $\psi$ is thermodynamically preferred below a critical value of $T / \mu$, indicating that the dual operator $O$ acquires an expectation value $\langle O\rangle$. For simplicity, let us focus on s-wave superconductors with a scalar order parameter $\langle O\rangle$ so that $\psi$ is a scalar field. The trivial solution $\psi=0$ for a scalar field becomes unstable if its effective mass $m_{\text {eff }}^{2}$ becomes sufficiently tachyonic. In an $A d S_{d+1}$ space with radius $L$ this means that $m_{\text {eff }}^{2}$ has to violate the BF bound (2.20), i.e. $m_{\mathrm{eff}}^{2} L^{2}<-d^{2} / 4$. The effective mass of the charged scalar $\psi$ is composed of the quadratic term $m^{2}$ from its bulk potential and of a negative contribution from the non-trivial Maxwell field $A_{t}, m_{\text {eff }}^{2}=m^{2}+g^{t t} A_{t} A_{t}$ [103]. As $A_{t}$ becomes bigger, i.e. as $T / \mu$ is lowered, $m_{\text {eff }}^{2}$ becomes smaller. Eventually, below a critical value of $T / \mu$, it violates the BF bound. If this violation persists in a sufficiently large region of the bulk, $\psi$ spontaneously develops a non-trivial profile [71,99,103].

The same condensation mechanism is at work in the holographic Kondo model discussed in chapter 5. There, the Maxwell field is confined to a two-dimensional bulk, however, as it is dual to the charge of a localised impurity rather than to an 
electromagnetic current. Consequently, no electrical conductivity can be associated with it.

In higher dimensions on the other hand, a perturbation by an electromagnetic field $A_{x}(t)$ indeed results in a current of the London form $J_{x} \sim-\nu A_{x}$ below the critical temperature, with $\nu \sim\langle O\rangle[103,106,107]$. If the holographic theory has translational invariance, that is, if the charge carriers' momentum is conserved, then $\nu$ receives another $T$-independent contribution unrelated to superconductivity, as expected from our discussion in the previous subsection. The simplest albeit crude way to break translational symmetry, i.e. to break the dual diffeomorphism invariance of the bulk, is to ignore the backreaction of $A_{\mu}$ and $\psi$ on the bulk metric and work in a fixed AdS black-brane background [71,99]. This effectively allows the charged probe sector dual to $A_{\mu}$ and $\psi$ to dissipate momentum into the uncharged degrees of freedom. The same effect can be seen in probe-brane systems [108].

We should note that the photon in holographic superconductors is not dynamical: the $U(1)$ gauge field in the bulk couples to a global $U(1)$ current in the dual field theory. Technically, we therefore cannot distinguish between holographic superconductors and holographic superfluids [71,99, 103]. However, for the production of a diamagnetic London current (3.37), and in fact for many condensed matter processes, interactions of the photon do not play an important role [71].

\subsection{Spectral function and dissipation}

\subsubsection{Classical dissipation}

In section 3.1 we saw that the eigenfrequencies of the damped harmonic oscillator show up as peaks in $\operatorname{Im} G_{R}(\omega)$. Indeed, we will now see that $\operatorname{Im} G_{R}(\omega)$ generally describes a system's spectrum of energy absorption, i.e. it controls how much energy can be dissipated into the system by a periodic driving force with frequency $\omega$. The work done on a classical system per unit time by an external force $F(t)$ is

$$
\begin{aligned}
\frac{\mathrm{d} W}{\mathrm{~d} t} & =F(t) \dot{x}(t) \\
& =-\int \frac{\mathrm{d} \omega}{2 \pi} e^{-i \omega t} F(\omega) \int \frac{\mathrm{d} \omega^{\prime}}{2 \pi}\left(-i \omega^{\prime}\right) e^{-i \omega^{\prime} t} G_{R}\left(\omega^{\prime}\right) F\left(\omega^{\prime}\right) .
\end{aligned}
$$


Specifying to a periodic force

$$
F(t)=2 F_{0} \cos (\Omega t)
$$

and averaging over a cycle we find

$$
\begin{aligned}
\frac{\Omega}{2 \pi} \int_{0}^{2 \pi / \Omega} \mathrm{d} t \frac{\mathrm{d} W}{\mathrm{~d} t} & =i F_{0}^{2} \Omega\left(G_{R}(\Omega)-G_{R}(-\Omega)\right) \\
& =F_{0}^{2} \Omega\left(-2 \operatorname{Im} G_{R}(\Omega)\right)
\end{aligned}
$$

In the last line, we used that the Green's function $G_{R}(t)$, which relates a real source $F(t)$ to a real response $x(t)$, must itself be real, hence $G_{R}(\omega)^{*}=G_{R}(-\omega)$.

The average energy dissipated by $F$ into the system over a full cycle cannot be negative. The spectral function

$$
\rho(\omega) \equiv-2 \operatorname{Im} G_{R}(\omega)
$$

must therefore satisfy

$$
\omega \rho(\omega) \geq 0
$$

\subsubsection{Quantum dissipation}

We will now show that $\operatorname{Im} G_{R}(k)$ also controls the absorption in quantum systems [71, 87]. For simplicity, we will perturb the system by a single Hermitian operator $O(x)=$ $O(x)^{\dagger}$, coupled to a real source $\phi(x)$. Working in the Schrödinger picture, the energy dissipated into the system by $\phi$ is

$$
\begin{aligned}
\frac{\mathrm{d} W}{\mathrm{~d} t} & =\frac{\mathrm{d}}{\mathrm{d} t} \operatorname{Tr}(\rho H)=\operatorname{Tr}(-i[H, \rho] H+\rho \dot{\delta} \dot{H}) \\
& =-\operatorname{Tr}\left(\rho \int \mathrm{d} \underline{x} O(\underline{x}) \partial_{t} \phi(x)\right) \\
& =-\int \mathrm{d} \underline{x}\langle O(x)\rangle \partial_{t} \phi(x) .
\end{aligned}
$$

Only taking into account the linear response (3.19) of $\langle O\rangle$ to $\phi$, choosing a periodic source

$$
\phi(x)=2 \operatorname{Re}\left(\phi_{0}(\underline{x}) e^{-i \Omega t}\right)
$$


and averaging over a cycle yields

$$
\frac{\Omega}{2 \pi} \int_{0}^{2 \pi / \Omega} \mathrm{d} t \frac{\mathrm{d} W}{\mathrm{~d} t}=i \Omega \int \frac{\mathrm{d} \underline{k}}{(2 \pi)^{d-1}}\left|\phi_{0}(\underline{k})\right|^{2}\left(G_{R}(\Omega, \underline{k})-G_{R}(-\Omega,-\underline{k})\right) .
$$

A Hermitian operator $O(x)$ has a real expectation value $\langle O(x)\rangle$ and, if the Hamiltonian is to be Hermitian, must be coupled to a real source $\phi(x) .^{1}$ The associated Green's function $G_{R}(x)$ must hence be real too, $G_{R}(k)^{*}=G_{R}(-k)$, and the absorbed energy (3.50) is again controlled by the spectral function

$$
\rho(k) \equiv-2 \operatorname{Im} G_{R}(k),
$$

which must satisfy

$$
\omega \rho(\omega, \underline{k}) \geq 0 \text {. }
$$

Other useful properties of causal Green's functions and spectral functions are summarised in appendix A.

In quantum systems with a discrete energy spectrum, the spectral function reduces to a sum of delta functions, much like the spectrum $\rho(w)=-2 \operatorname{Im} G_{R}(\omega)$ of the classical harmonic oscillator in the frictionless, "unitary" limit $\gamma \rightarrow 0^{+}$, eq. (3.11). This can be seen explicitly in the spectral representation of $G_{R}(k)$ in terms of energy eigenstates [71, 87,109]. If a system contains unstable states with a finite lifetime $\tau$ and accordingly with an "uncertain" energy, then these states will show up in the spectrum as smooth poles with width $\sim 1 / \tau$, analogous to the resonances with width $\gamma$ in the spectrum of the damped harmonic oscillator.

\subsubsection{Fano resonances}

We saw in section 3.1 that a low-lying pole of $G_{R}$ with a real residue, eq. (3.7), leads to a symmetric Lorentzian peak in the spectral function $\rho=-2 \operatorname{Im} G_{R}$, eq. (3.9). What

\footnotetext{
${ }^{1}$ There is nothing preventing us from coupling a Hermitian operator $O\left(\operatorname{such}\right.$ as $T^{\mu \nu}$ ) to a convenient complex source (such as $h_{\mu \nu} \sim H_{\mu \nu} e^{i k x}$ ) if we are only interested in computing $G_{R}(k)$. The response $\delta\langle O(x)\rangle$ will not be real in that case, but $G_{R}(k)$ itself is a property of the unperturbed state alone and as such is unaffected by our choice of source.
} 
happens near a pole at $\omega=\omega_{*}, G_{R} \sim Z /\left(\omega-\omega_{*}\right)$, with a complex residue $Z$ ? Defining $\tilde{\omega} \equiv \operatorname{Re} \omega_{*}$ and $\Gamma / 2 \equiv-\operatorname{Im} \omega_{*}$, and introducing the asymmetry parameter

$$
q \equiv-\frac{\operatorname{Re} Z+\sqrt{(\operatorname{Re} Z)^{2}+(\operatorname{Im} Z)^{2}}}{\operatorname{Im} Z}
$$

the spectral function $\rho=-2 \operatorname{Im} G_{R}$ takes the form of a Fano resonance, that is, it can be written as

$$
\rho=-\frac{\operatorname{Im} Z}{q(\Gamma / 2)}\left(\rho_{\text {Fano }}-1\right)
$$

with the Fano spectral function

$$
\begin{aligned}
\rho_{\text {Fano }}(\omega) & \equiv \frac{(\omega-\tilde{\omega}+q \Gamma / 2)^{2}}{(\omega-\tilde{\omega})^{2}+(\Gamma / 2)^{2}} \\
& =1+\left(q^{2}-1\right) \frac{(\Gamma / 2)^{2}}{(\omega-\tilde{\omega})^{2}+(\Gamma / 2)^{2}}+2 q \frac{(\Gamma / 2)(\omega-\tilde{\omega})}{(\omega-\tilde{\omega})^{2}+(\Gamma / 2)^{2}} .
\end{aligned}
$$

The last line reveals that $\rho_{\text {Fano }}$ is the sum of a constant piece (representing a featureless continuum), a Lorentzian resonance, and a mixing term. Indeed, Fano resonances [110] arise when a continuum of energy states couples to a resonance. Incoming scattering states, from the continuum, then have two paths through the system: They can either scatter off the resonant state (resonant scattering) or they can bypass the resonant state (non-resonant scattering) [111]. For generic $q, \rho_{\text {Fano }}(\omega)$ is asymmetric with respect to $\tilde{\omega}$, with a minimum $\rho_{\text {Fano }}=0$ at $\omega=\tilde{\omega}-q(\Gamma / 2)$ and a maximum $\rho_{\text {Fano }}=1+q^{2}$ at $\omega=\tilde{\omega}+\frac{1}{q}(\Gamma / 2)$. In the limit $q \rightarrow \pm \infty$, the minimum disappears and $\rho_{\text {Fano }}(\omega)$ reduces to a symmetric Lorentzian centred around $\omega=\tilde{\omega}$. For $q \rightarrow 0$, the maximum disappears and $\rho_{\text {Fano }}(\omega)$ becomes a symmetric dip or anti-resonance. This suggests that $|q|$ measures the amount of resonant scattering. Indeed, $q^{2}$, as originally introduced by Fano, is proportional to the ratio of probabilities for resonant versus non-resonant scattering [110].

One obtains a simple classical realisation of a Fano resonance by weakly coupling a classical damped harmonic oscillator $x(t)$ (providing a continuous spectrum) to a second undamped oscillator (providing a sharp resonance) [112]:

$$
\begin{aligned}
\ddot{x}(t)+\gamma \dot{x}(t)+\omega_{0}^{2} x(t)+\kappa y(t) & =0, \\
\ddot{y}(t)+\Omega_{0}^{2} y(t)+\kappa x(t) & =0 .
\end{aligned}
$$


Coupling the damped oscillator $x$ to an external driving source, we find that its retarded Green's function is given by

$$
G_{R}(\omega)=\frac{\omega^{2}-\Omega_{0}^{2}}{\left(\omega^{2}+i \gamma \omega-\omega_{0}^{2}\right)\left(\omega^{2}-\Omega_{0}^{2}\right)-\kappa^{2}} .
$$

Note that it vanishes at the eigenfrequency $\omega= \pm \Omega_{0}$ of the sharp resonance as a result of perfect destructive interference between the coupled sharp resonance and the external source [111]. This destructive interference is also responsible for the minimum in $\rho_{\text {Fano. }}$. Constructive interference, by contrast, results in a modified resonant state, corresponding to a pole in $G_{R}(\omega)$ at the slightly shifted position

$$
\omega_{*}=\Omega_{0}+\delta \Omega_{+},
$$

and similarly around $-\Omega_{0}$. To leading order in the coupling $\kappa$ we find

$$
\delta \Omega_{+}=\frac{\kappa^{2}}{2 \Omega_{0}\left(\Omega_{0}^{2}+i \gamma \Omega_{0}-\omega_{0}^{2}\right)} .
$$

Assuming that $\Omega_{0}$ and $\omega_{0}$ are well separated so that the damped oscillator contributes a more or less featureless continuum around $\operatorname{Re} \omega_{*}$, we find that $G_{R}(\omega)$ indeed takes the form $Z /\left(\omega-\omega_{*}\right)$ near the resonant state $\omega=\omega_{*}$, with a complex residue

$$
Z=\frac{\kappa^{2}}{2 \Omega_{0}} \frac{1}{\left(\Omega_{0}^{2}+i \gamma \Omega_{0}-\omega_{0}^{2}\right)^{2}}+\mathcal{O}\left(\kappa^{4}\right) .
$$

Accordingly, the spectrum $\rho=-2 \operatorname{Im} G_{R}$ exhibits an asymmetric Fano resonance.

We also observe Fano resonances in the holographic Kondo model in chapter 5. This model describes a holographic RG flow at a magnetic impurity localised in $(0+1)$ dimensions. At low temperatures, a scalar operator $\mathcal{O}$ condenses, representing the formation of a Kondo cloud of charge carriers bound to the impurity. The spectral function of $\mathcal{O}$ inherits $(0+1)$-dimensional scale invariance from the UV fixed point, which results in a continuous spectrum. However, the Kondo coupling, which triggers the RG flow, introduces a dynamically generated energy scale and produces a resonance. The interference of this resonance with the continuum creates a Fano resonance in $\mathcal{O}$ 's spectral function. 


\section{Chapter 4}

\section{Second-Order Hydrodynamics and Universality in Non-Conformal Holographic Fluids}

\subsection{Introduction and summary}

In section 3.2 we introduced hydrodynamics [90,91] as the low-energy effective theory for slowly varying fluctuations around thermal equilibrium. Considering the simplest case of an uncharged relativistic fluid in $(3+1)$ dimensions we obtained the constitutive relation of the fluid stress tensor to first order in the gradient expansion in eq. (3.23). At second order, the constitutive relation features another fifteen transport coefficients $[10,93]$. Kubo formulae, which tell us which correlators exactly to look at in order to compute a specific transport coefficient [87,90], are known for both first-order coefficients $\eta$ and $\zeta$, for the five second-order coefficients already present in conformal fluids [113], and for six of the ten second-order coefficients only present in non-conformal fluids [114].

In recent years, hydrodynamics has been successfully applied to describe the earlystage evolution of the quark-gluon plasma (QGP) created in heavy-ion collisions at RHIC [115-119]. In fact, it captures the evolution of the QGP from surprisingly early times onwards. However, it is not sufficient to simulate the QGP with first-order viscous hydrodynamics, as this is plagued by superluminal modes which violate causality. To get rid of these modes it is necessary to take into account second-order terms as well [10]. As the temperature of the QGP is not too far from the confinement scale of QCD, the 
QGP falls into the intermediate-coupling regime and its transport coefficients cannot be computed by perturbative methods. Lattice calculations are not well-suited to computations of transport coefficients either: computations of real-time correlators face the formidable problem of analytic continuation whereas indirect methods are plagued with uncertainties [120].

The only currently available tool that allows for the computation of real-time correlators in strongly interacting field theories is gauge/gravity duality $[1-4,47,73]$. In particular, it reduces computing stress-tensor correlators in the hydrodynamic regime to solving classical Einstein's equations on an AdS background in a small-momentum expansion. Applying the appropriate Kubo formulae, one can read off the transport coefficients in the strongly coupled holographic field theory [94-96]. Apart from its relevance for strongly coupled field theories, a hydrodynamic interpretation of solutions to Einstein's equations is interesting in its own right, because it extends the analogy between thermodynamics and black-hole mechanics beyond thermal equilibrium to a more general correspondence between fluids and gravity [11].

At present, the construction of the exact holographic dual of realistic theories such as QCD is beyond reach. The best one can hope for when trying to make connections with experiment is to identify and investigate properties that hold for a large class of holographic models: being insensitive to the microscopic details of the dual field theory, it is possible that such properties are shared by many or even all QFTs in the strong coupling limit, including the ones realised in nature. The most prominent example of such a universal property is the ratio of shear viscosity $\eta$ over entropy density $s$ [43] which is known to obey $\eta / s=1 / 4 \pi$ for any strongly coupled theory with a twoderivative gravity dual and unbroken $\mathrm{SO}(3)$ rotational symmetry [7, 67, 94, 121-126]. Current estimates for the value of $\eta / s$ in the QGP extracted from heavy ion collision data are indeed close to $1 / 4 \pi[127-133]$.

Universality in hydrodynamic transport is most likely to be observed among transport coefficients that can be measured without considering sound perturbations, which would necessarily excite the model-specific matter content [134]. There exists one particular relation between second-order transport coefficients which promises to exhibit 
such universal behaviour:

$$
H \equiv 2 \eta \tau_{\pi}-4 \lambda_{1}-\lambda_{2}=0
$$

Prompted by the observation in ref. [135], Haack and Yarom [14] demonstrated that the combination $H$ vanishes for conformal holographic theories with a two-derivative gravity dual, and with any number of $U(1)$-charges at finite density. It has further been shown that $H$ remains zero when taking into account leading corrections to the infinite coupling limit, both in planar $\mathcal{N}=4[136]$ and in the hypothetical fluid dual to Gauss-Bonnet gravity [137-139]. A simple example of non-conformal holographic transport was studied in ref. [140]: employing the method developed in ref. [141] and working to linear order in $1 / 3-c_{s}^{2}$ with speed of sound $c_{s}$, the authors showed that $H$ also vanishes for the non-conformal Chamblin-Reall background. This background, however, can simply be viewed as the analytic continuation of higher-dimensional AdS compactified on a torus [142]. While the values of the transport coefficients in this special non-conformal model do differ from the conformal values, they are nonetheless completely dictated by the higher-dimensional conformal theory [141]. In particular, the five coefficients that are already present in the conformal case are simply multiplied by a common factor so that relations that hold between coefficients in the conformal case, such as $H=0$, trivially apply to this non-conformal compactification as well. The only holographic model in which non-conformal corrections to $H$ have been taken into account beyond leading order are the compactified D4-branes of ref. [143], where the relation $H=0$ was also found to hold. ${ }^{1}$ Note that neither the Chamblin-Reall background studied in ref. [140] nor the compactified branes of ref. [143] admit an asymptotically AdS region. ${ }^{2}$ Their holographic duals therefore do not have an obvious UV fixed point.

Whether $H=0$ holds more generally in holographic theories without conformal symmetry remains an open question which we want to address in this chapter, based on ref. [13]. To this end we compute the second-order transport coefficients entering

\footnotetext{
${ }^{1}$ Other recent holographic and non-holographic studies of second-order transport in non-conformal relativistic fluids include refs. [144-150].

${ }^{2}$ In both cases the bulk geometry can be viewed as a compactification of AdS and one can essentially borrow the higher dimensional AdS/CFT dictionary [151,152].
} 
$H$ for a large class of non-conformal holographic models from three-point functions of the stress tensor. The precise type of models we consider are holographic RG flows in asymptotically $A d S_{5}$, triggered by an arbitrary scalar operator of dimension $\Delta=3$. We prove analytically that $H$ vanishes for this class of models even when leading nonconformal corrections to the transport coefficients are included. We subsequently study two specific families of $\mathrm{RG}$ flows from that class and show numerically that $H$ vanishes along both.

This chapter is structured as follows: in section 4.2 we derive new Kubo formulae for the five second-order coefficients

$$
\kappa, \quad \eta \tau_{\pi}+\kappa^{*}, \quad \lambda_{1}+\frac{\kappa^{*}}{2}, \quad \lambda_{2}, \quad \lambda_{3}-2 \kappa^{*}
$$

by considering the response of the stress tensor to shear perturbations of the external metric. The coefficients (4.2) are combinations of the five coefficients present in conformal fluids and the non-conformal coefficient $\kappa^{*}$. The combination $H$, eq. (4.1), can be obtained as a linear combination of these coefficients. The Kubo formulae we derive are valid for any uncharged relativistic fluid in four dimensions, with or without conformal symmetry. We end section 4.2 with a brief explanation of how they can be applied specifically to theories with a holographic dual. In section 4.3 we introduce the class of strongly coupled non-conformal models studied in this chapter. These are four-dimensional holographic theories with a UV fixed point, deformed by a relevant scalar operator of dimension $\Delta=3$. The dual description of such $\mathrm{RG}$ flows is provided by Einstein gravity in asymptotically $A d S_{5}$, coupled to a scalar field with mass $m^{2} L^{2}=\Delta(\Delta-4)=-3$ but with an otherwise arbitrary potential. We derive the relevant bulk equations of motion for black-brane backgrounds and for metric perturbations. In section 4.4 we solve Einstein's equations for bulk metric perturbations around a general black-brane background. Section 4.5 contains our analytical results on second-order transport in the class of non-conformal holographic models that we investigate. We present formulae for the five second-order coefficients (4.2) for a given background solution in subsection 4.5.1. The coefficients always satisfy the relation $\tilde{H} \equiv 2 \eta \tau_{\pi}-2\left(\kappa-\kappa^{*}\right)-\lambda_{2}=0$. In subsection 4.5 .2 we prove analytically that $H$ remains zero when leading non-conformal corrections to the transport coefficients are 
taken into account. Section 4.6 contains our numerical results. Treating the bulk scalar field as a small perturbation, we obtain, in subsection 4.6.1, the leading non-conformal corrections to the transport coefficients in the vicinity of the UV fixed point. These leading corrections only depend on the mass term in the scalar potential and therefore apply to all holographic RG flows triggered by a scalar operator of dimension $\Delta=3$. In subsection 4.6 .2 we introduce two specific families of bulk potentials: the first one describes RG flows to a fixed point in the IR, the second one describes flows to a nonconformal IR. We present our numerical results for the five transport coefficients in subsection 4.6.3. Our main result is that the combination $H$, eq. (4.1), vanishes for both families of flows, even if the individual transport coefficients deviate from their conformal values by factors of two and more. We mention how our results are extended to eight second-order coefficients by employing relations which must hold if the local entropy production is to be non-negative under all circumstances. We conclude with a summary of our results and propose future directions of research in section 4.7. We attach technical details of our calculations in appendix B.

\subsection{New Kubo formulae for non-conformal second- order hydrodynamics}

We derive a new set of Kubo formulae (4.21) for five second-order transport coefficients in subsection 4.2.1. These Kubo formulae are valid for any uncharged relativistic fluid in $(3+1)$ dimensions, with or without conformal symmetry, and constitute one of the main results of this chapter. In subsection 4.2 .2 we outline how they can be applied specifically to strongly coupled fluids with a holographic gravity dual.

\subsubsection{Sourced fluid stress tensor and Kubo formulae}

The constitutive relations for an uncharged relativistic fluid are known up to third order in gradients $[10,93,153]$. Up to second order, seventeen independent tensor structures can be constructed from the fluid variables and $g_{(0) \mu \nu}$, each multiplied by a transport coefficient such as shear viscosity (see appendix B.1 for the explicit expressions). These 
transport coefficients are the free input parameters of the effective hydrodynamic description. In order to compute their values we have to match the hydro result for appropriate correlators of $\left\langle T^{\mu \nu}\right\rangle$ with the corresponding result in the underlying microscopic theory.

A suitable quantity to match is the response of $\left\langle T^{\mu \nu}\right\rangle$ to an external metric perturbation around flat space of a fluid in equilibrium. In the equilibrium rest frame, the fluid variables of the perturbed fluid on the background $g_{(0) \mu \nu}(x)=\eta_{\mu \nu}+h_{\mu \nu}(x)$ will take the form

$$
\epsilon(x)=\bar{\epsilon}+\delta \epsilon(x), \quad u^{\mu}(x)=(1, \underline{v})\left(-g_{(0) t t}-2 g_{(0) t i} v^{i}-g_{(0) i j} v^{i} v^{j}\right)^{-1 / 2} .
$$

It is convenient to use $\delta \epsilon(x)$ and $\underline{v}(x)$ as fluid variables as this allows for a second expansion in fluctuations around static global equilibrium sourced by $h_{\rho \sigma}$, in addition to the hydro gradient expansion. Writing the equilibrium stress tensor as

$$
\bar{T}^{\mu \nu} \equiv\left\langle T^{\mu \nu}\right\rangle\left[\delta \epsilon=\underline{v}=h_{\rho \sigma}=0\right],
$$

the off-shell stress tensor of the perturbed fluid assumes the following form to first order $\mathcal{O}(\delta)$ in fluctuations:

$$
\left\langle T^{\mu \nu}\right\rangle\left[\delta \epsilon, \underline{v} ; h_{\rho \sigma}\right]=\bar{T}^{\mu \nu}+\left[\frac{\partial \bar{T}^{\mu \nu}}{\partial \delta \epsilon} \delta \epsilon+\frac{\partial \bar{T}^{\mu \nu}}{\partial v^{i}} v^{i}\right]+\frac{\partial \bar{T}^{\mu \nu}}{\partial h_{\rho \sigma}} h_{\rho \sigma}+\mathcal{O}\left(\delta^{2}\right)
$$

where we defined

$$
\left.\frac{\partial \bar{T}^{\mu \nu}}{\partial \delta \epsilon} \equiv \frac{\partial\left\langle T^{\mu \nu}\right\rangle}{\partial \delta \epsilon}\right|_{\delta \epsilon=\underline{v}=h_{\rho \sigma}=0}
$$

etc. Linearising the conservation eq. (3.20) around equilibrium yields the equations of motion for the fluid variables $\delta \epsilon$ and $\underline{v}$ in the presence of the linear metric perturbation $h_{\rho \sigma}$. Defining

$$
\delta T_{(\delta \epsilon, \underline{v})}^{\mu \nu} \equiv \frac{\partial \bar{T}^{\mu \nu}}{\partial \delta \epsilon} \delta \epsilon+\frac{\partial \bar{T}^{\mu \nu}}{\partial v^{i}} v^{i}, \quad \delta T_{(h)}^{\mu \nu} \equiv \frac{\partial \bar{T}^{\mu \nu}}{\partial h_{\rho \sigma}} h_{\rho \sigma},
$$

these equations read

$$
\partial_{\mu} \delta T_{(\delta \epsilon, \underline{v})}^{\mu \nu}=-\partial_{\mu} \delta T_{(h)}^{\mu \nu}-\delta \Gamma_{\mu \rho}^{\mu} \bar{T}^{\rho \nu}-\delta \Gamma_{\mu \rho}^{\nu} \bar{T}^{\mu \rho}+\mathcal{O}\left(\delta^{2}\right)
$$


As $h_{\rho \sigma}$ sources hydro fluctuations around static equilibrium we impose the boundary condition that $\delta \epsilon=\underline{v}=0$ for $h=0$, i.e. we do not consider the usual free hydro modes that solve eq. (4.8) in the absence of the source terms on the right-hand side. There exists a particularly simple subset of non-trivial metric perturbations in four dimensions $x^{\mu}=(t, x, y, z)$ that do not source any fluctuations of the fluid variables to first order. Explicitly, if we only turn on

$$
\left\{h_{x y}(t, z), h_{t x}(z), h_{t y}(z), h_{x z}(t), h_{y z}(t)\right\}
$$

then the right-hand side of eq. (4.8) vanishes at $\mathcal{O}(h)$, and the hydro equations are solved by $\delta \epsilon(h), \underline{v}(h)=\mathcal{O}\left(h^{2}\right)$. In that case, the on-shell stress tensor takes the following form to second order in the source $\mathcal{O}\left(h^{2}\right)$ :

$$
\begin{aligned}
\left\langle T^{\mu \nu}\right\rangle\left[h_{\rho \sigma}\right]= & \bar{T}^{\mu \nu}+\frac{\partial \bar{T}^{\mu \nu}}{\partial h_{\rho \sigma}} h_{\rho \sigma}+\frac{1}{2} \frac{\partial^{2} \bar{T}^{\mu \nu}}{\partial h_{\rho \sigma} \partial h_{\kappa \lambda}} h_{\rho \sigma} h_{\kappa \lambda} \\
& +\left(\frac{\partial \bar{T}^{\mu \nu}}{\partial \delta \epsilon} \delta \epsilon(h)+\frac{\partial \bar{T}^{\mu \nu}}{\partial v^{i}} v^{i}(h)\right)+\mathcal{O}\left(h^{3}\right) .
\end{aligned}
$$

This expression simplifies even further if we focus on the transverse-tensor component $\left\langle T^{x y}(t, z)\right\rangle$ as it is independent of the scalars $\delta \epsilon, v^{z}$ and the transverse-vector components $v^{x}, v^{y}$ to first order $\mathcal{O}(\delta)$ :

$$
\frac{\partial \bar{T}^{x y}}{\partial \delta \epsilon}=\frac{\partial \bar{T}^{x y}}{\partial v^{i}}=0 .
$$

Using this together with the constitutive relation, the on-shell response of $\left\langle T^{x y}\right\rangle$ to the metric perturbations $(4.9)$ at $\mathcal{O}\left(h^{2}\right)$ is found to be

$$
\begin{aligned}
\left\langle T^{x y}\right\rangle= & {\left[-\bar{p}-\eta \partial_{t}-\frac{\kappa}{2} \partial_{z}^{2}+\left(\eta \tau_{\pi}-\frac{\kappa}{2}+\kappa^{*}\right) \partial_{t}^{2}\right] h_{x y}(t, z) } \\
+ & {\left[\bar{p} h_{x z} h_{y z}+\eta\left(h_{x z} \partial_{t} h_{y z}+\partial_{t} h_{x z} h_{y z}\right)+\left(\lambda_{1}-\eta \tau_{\pi}-\frac{\kappa^{*}}{2}\right) \partial_{t} h_{x z} \partial_{t} h_{y z}\right.} \\
& \left.+\left(\frac{\kappa}{2}-\eta \tau_{\pi}-\kappa^{*}\right)\left(h_{x z} \partial_{t}^{2} h_{y z}+\partial_{t}^{2} h_{x z} h_{y z}\right)\right] \\
+ & {\left[-\bar{p} h_{t x} h_{t y}+\left(\frac{\lambda_{3}}{4}-\frac{\kappa^{*}}{2}\right) \partial_{z} h_{t x} \partial_{z} h_{t y}-\frac{\kappa}{2}\left(h_{t x} \partial_{z}^{2} h_{t y}+\partial_{z}^{2} h_{t x} h_{t y}\right)\right] } \\
+ & {\left[\frac{1}{2} \eta \tau_{\pi}-\frac{\lambda_{2}}{4}+\frac{\kappa^{*}}{2}\right]\left(\partial_{z} h_{t x} \partial_{t} h_{y z}+\partial_{z} h_{t y} \partial_{t} h_{x z}\right)+\mathcal{O}\left(h^{3}, \partial^{3}\right) }
\end{aligned}
$$

where $\bar{p}$ denotes the pressure in global equilibrium. The linear response sourced by the tensor perturbation $h_{x y}$ was derived in ref. [10], the quadratic response sourced by 
the transverse-vector perturbations was computed in ref. [113] for a conformal fluid with $\kappa^{*}=0$. To our knowledge, the response (4.12) of a non-conformal fluid has not appeared in the literature before. Note in particular that $\kappa^{*}$ is the only one of the ten non-conformal second-order coefficient that appears in eq. (4.12). Eq. (4.12) shows that the response of $\left\langle T^{x y}\right\rangle$ to the perturbations (4.9) gives us access to five independent linear combinations of second-order transport coefficients, ${ }^{3}$

$$
\kappa, \quad \eta \tau_{\pi}+\kappa^{*}, \quad \lambda_{1}+\frac{\kappa^{*}}{2}, \quad \lambda_{2}, \quad \lambda_{3}-2 \kappa^{*},
$$

which includes the combination $H=2 \eta \tau_{\pi}-4 \lambda_{1}-\lambda_{2}$. In fact, no additional information is provided by the response to $h_{x y}$, and all five coefficients (4.13) can be obtained by turning on plane-wave excitations for $\left\{h_{x z}(t), h_{y z}(t)\right\},\left\{h_{t x}(z), h_{t y}(z)\right\}$, and $\left\{h_{t y}(z), h_{x z}(t)\right\}$, one after another.

Turning on $\left\{h_{x z}(t), h_{y z}(t)\right\}$ : Perturbing the metric by

$$
\frac{1}{2} h_{\mu \nu} \mathrm{d} x^{\mu} \mathrm{d} x^{\nu}=\epsilon\left(H_{x z}^{(b)} e^{-i q_{0} t} \mathrm{~d} x \mathrm{~d} z+H_{y z}^{(b)} e^{-i p_{0} t} \mathrm{~d} y \mathrm{~d} z\right),
$$

with plane-wave amplitudes $H_{x z}^{(b)}$ and $H_{y z}^{(b)}$, sources the response

$$
\begin{aligned}
\left\langle T^{x y}(x)\right\rangle= & {\left[\bar{p}-i\left(q_{0}+p_{0}\right) \eta-q_{0} p_{0}\left(\lambda_{1}-\eta \tau_{\pi}-\frac{\kappa^{*}}{2}\right)\right.} \\
& \left.-\left(q_{0}^{2}+p_{0}^{2}\right)\left(\frac{\kappa}{2}-\eta \tau_{\pi}-\kappa^{*}\right)\right] \epsilon^{2} H_{x z}^{(b)} H_{y z}^{(b)} e^{-i\left(q_{0}+p_{0}\right) t}+\mathcal{O}\left(\epsilon^{3}, \partial^{3}\right),
\end{aligned}
$$

corresponding to the second and third line in eq. (4.12).

Turning on $\left\{h_{t x}(z), h_{t y}(z)\right\}$ : Perturbing the metric by

$$
\frac{1}{2} h_{\mu \nu} \mathrm{d} x^{\mu} \mathrm{d} x^{\nu}=\epsilon\left(H_{t x}^{(b)} e^{i q_{z} z} \mathrm{~d} t \mathrm{~d} x+H_{t y}^{(b)} e^{i p_{z} z} \mathrm{~d} t \mathrm{~d} y\right),
$$

with plane-wave amplitudes $H_{t x}^{(b)}$ and $H_{t y}^{(b)}$, sources the response

$$
\begin{aligned}
\left\langle T^{x y}(x)\right\rangle= & {\left[-\bar{p}-q_{z} p_{z}\left(\frac{\lambda_{3}}{4}-\frac{\kappa^{*}}{2}\right)+\left(q_{z}^{2}+p_{z}^{2}\right) \frac{\kappa}{2}\right] \epsilon^{2} H_{t x}^{(b)} H_{t y}^{(b)} e^{i\left(q_{z}+p_{z}\right) z} } \\
& +\mathcal{O}\left(\epsilon^{3}, \partial^{3}\right)
\end{aligned}
$$

corresponding to the fourth line in eq. (4.12).

\footnotetext{
${ }^{3}$ Note that if we wanted to extract all fifteen second-order coefficients we would have to turn on metric perturbations in the scalar sound channel which would necessarily source fluctuations of $\delta \epsilon$ and $\underline{v}$.
} 
Turning on $\left\{h_{t y}(z), h_{x z}(t)\right\}$ : Perturbing the metric by

$$
\frac{1}{2} h_{\mu \nu} \mathrm{d} x^{\mu} \mathrm{d} x^{\nu}=\epsilon\left(H_{t y}^{(b)} e^{i p_{z} z} \mathrm{~d} t \mathrm{~d} y+H_{x z}^{(b)} e^{-i q_{0} t} \mathrm{~d} x \mathrm{~d} z\right)
$$

with plane-wave amplitudes $H_{t y}^{(b)}$ and $H_{x z}^{(b)}$, sources the response

$$
\left\langle T^{x y}(x)\right\rangle=q_{0} p_{z}\left(\frac{1}{2} \eta \tau_{\pi}-\frac{\lambda_{2}}{4}+\frac{\kappa^{*}}{2}\right) \epsilon^{2} H_{t y}^{(b)} H_{x z}^{(b)} e^{-i q_{0} t+i p_{z} z}+\mathcal{O}\left(\epsilon^{3}, \partial^{3}\right),
$$

corresponding to the last line in eq. (4.12).

Kubo formulae: Comparing eqs. (4.15), (4.17), (4.19) with the general form of the stress-tensor response written in terms of retarded correlators in momentum space [113],

$$
\begin{aligned}
\left\langle T^{\mu \nu}(x=0)\right\rangle= & G^{\mu \nu}(0)-\frac{1}{2} \int \frac{\mathrm{d}^{4} p}{(2 \pi)^{4}} G^{\mu \nu, \rho \sigma}(p) h_{\rho \sigma}(p) \\
& +\frac{1}{8} \int \frac{\mathrm{d}^{4} q}{(2 \pi)^{4}} \frac{\mathrm{d}^{4} p}{(2 \pi)^{4}} G^{\mu \nu, \rho \sigma, \kappa \lambda}(q, p) h_{\rho \sigma}(q) h_{\kappa \lambda}(p)+\mathcal{O}\left(h^{3}\right),
\end{aligned}
$$

one can read off the low-momentum expansion of the corresponding three-point functions $G^{x y, x z, y z}, G^{x y, t x, t y}, G^{x y, t y, x z}$ and derive the following Kubo formulae: ${ }^{4}$

$$
\begin{aligned}
\kappa & =\left.\partial_{q_{z}}^{2} G^{x y, t x, t y}(q, p)\right|_{q=p=0}, \\
\eta \tau_{\pi}+\kappa^{*} & =\frac{\kappa}{2}+\left.\frac{1}{2} \partial_{q_{0}}^{2} G^{x y, x z, y z}(q, p)\right|_{q=p=0}, \\
\lambda_{1}+\frac{\kappa^{*}}{2} & =\left(\eta \tau_{\pi}+\kappa^{*}\right)-\left.\partial_{q_{0}} \partial_{p_{0}} G^{x y, x z, y z}(q, p)\right|_{q=p=0}, \\
\lambda_{2} & =2\left(\eta \tau_{\pi}+\kappa^{*}\right)-\left.4 \partial_{q_{0}} \partial_{p_{z}} G^{x y, t x, x z}(q, p)\right|_{q=p=0} \\
\lambda_{3}-2 \kappa^{*} & =-\left.4 \partial_{q_{z}} \partial_{p_{z}} G^{x y, t x, t y}(q, p)\right|_{q=p=0} .
\end{aligned}
$$

\subsubsection{Holographic calculation}

We now turn to the specific kind of microscopic theories that we will be studying throughout the remainder of this work: strongly coupled non-conformal QFTs with a holographic dual in asymptotically $A d S_{5}[4,38,105]$. In order to extract their secondorder transport coefficients we need to compute the stress-tensor component $\left\langle T^{x y}\right\rangle$ to

\footnotetext{
${ }^{4}$ Ref. [113] defines the Fourier-transformed three-point functions with the opposite sign for the two momenta. In our convention, the shear viscosity is therefore given by $\eta=\left.i \partial_{q_{0}} G^{x y, x z, y z}(q, p)\right|_{q=p=0}$, as opposed to eq. (21) in ref. [113], $\eta=-\left.i \partial_{q_{0}} G^{x y, x z, y z}(q, p)\right|_{q=p=0}$. We further believe that the factors of 2 in their eqs. (22) and (23) should be absent, in agreement with their eq. (26).
} 
second order $\mathcal{O}\left(\epsilon^{2}\right)$ in the perturbations (4.14), (4.16), (4.18) of the field-theory metric and match the result with the effective hydro results (4.15), (4.17), (4.19) [94,154,155]. Perturbations of the external field-theory metric act as boundary sources for perturbations of the dynamical bulk metric $g_{m n}$ in the dual gravity theory. Their backreaction on the bulk can be computed perturbatively in $\epsilon$. With regard to universality it is encouraging that, even for non-conformal fluids, $H$ can be measured by considering shear perturbations of the fluid. Unlike sound perturbations, shear perturbations only couple to the gravity sector of the dual bulk, which is common to all holographic theories, and not to the model-specific matter content [134].

According to the holographic dictionary $[2,3]$, the field-theory stress tensor $\left\langle T^{x y}\right\rangle$ equals, up to a scaling factor, the quasi-local gravity stress tensor $\mathcal{T}^{\mu \nu}$ of the dual AdS bulk [62] (see appendix C of ref. [13]). The latter measures the response of the on-shell gravity action to changes in the induced AdS boundary metric. Explicitly, variations of the induced AdS boundary metric $\gamma_{\mu \nu}$ lead to the following variation in the (appropriately renormalised) bulk action,

$$
\delta S^{\mathrm{ren}}=-\frac{1}{16 \pi G_{N}} \int \mathrm{d}^{5} x \sqrt{-g} \mathrm{EOM}^{m n} \delta g_{m n}+\frac{1}{2} \int_{\partial A d S_{5}} \mathrm{~d}^{4} x \sqrt{-\gamma} \mathcal{T}^{\mu \nu} \delta \gamma_{\mu \nu},
$$

where $\mathrm{EOM}_{m n}$ denote Einstein's equations in the bulk. Thus, for $(2 / \sqrt{-\gamma})\left(\delta S^{\mathrm{ren}} / \delta \gamma_{x y}\right)$ to yield the correct result for $\mathcal{T}^{x y}$ up to $\mathcal{O}\left(\epsilon^{2}\right)$ included, $\mathrm{EOM}^{x y}$ must be satisfied to order $\mathcal{O}\left(\epsilon^{2}\right)[154] .^{5}$

To summarise, our strategy to compute the transport coefficients will be the following: we turn on the boundary metric perturbations (4.14), (4.16), (4.18), one after another, solve the $x y$-component of Einstein's equations in the bulk to second order in amplitudes $\mathcal{O}\left(\epsilon^{2}\right)$ and momenta $\mathcal{O}\left(\partial^{2}\right)$ of the perturbations, compute the $x y$-component of the field-theory stress tensor according to the holographic dictionary, and finally compare it with the general hydro results (4.15), (4.17), (4.19).

\footnotetext{
${ }^{5}$ Given that the bulk metric will be diagonal to leading order, $g_{m n} \propto \delta_{m n}$, we can ensure that $\mathrm{EOM}^{x y}=\mathcal{O}\left(\epsilon^{3}\right)$ by solving the usual form of Einstein's equations with lower indices, $\mathrm{EOM}_{m n}$, to $\mathcal{O}(\epsilon)$ and $\mathrm{EOM}_{x y}$ to $\mathcal{O}\left(\epsilon^{2}\right)$.
} 


\subsection{A class of non-conformal holographic models}

In this section we introduce the specific class of strongly coupled non-conformal field theories, described by a holographic gravity dual, whose transport properties we are going to study: we will consider holographic RG flows [68,69,80,156-162] which, starting from a four-dimensional CFT in the UV, are triggered by a relevant deformation

$$
\int \mathrm{d}^{4} x \sqrt{-g_{(0)}} \Lambda(x) O(x)
$$

with a scalar operator $O$ of dimension $\Delta=3 .{ }^{6}$ The dual gravity description of such RG flows is provided by five-dimensional Einstein gravity coupled to a scalar field,

$$
S=\frac{1}{16 \pi G_{N}} \int \mathrm{d}^{5} x \sqrt{-g}\left[R-\frac{1}{2}(\partial \phi)^{2}-V(\phi)\right],
$$

with potentials of the form

$$
V(\phi)=\frac{1}{L^{2}}\left[-12-\frac{3}{2} \phi^{2}+\mathcal{O}\left(\phi^{4}\right)\right] .
$$

Solutions to the bulk equations of motion approach $A d S_{5}$ with radius $L$ in the nearboundary region $\phi \rightarrow 0$,

$$
\mathrm{d} s^{2} \rightarrow \frac{L^{2}}{\zeta^{2}}\left(\mathrm{~d} \zeta^{2}+\mathrm{d} x \cdot \mathrm{d} x\right)
$$

which is dual to the UV fixed point. The leading near-boundary mode of the scalar with mass $m^{2} L^{2}=\Delta(\Delta-4)=-3$ is $\phi \sim \Lambda \zeta$, where $\Lambda$ is interpreted as the source of the dual operator $O$.

\subsubsection{Background equations of motion}

Thermal equilibrium states in flat space are holographically described by black-brane solutions to (4.24) that preserve Euclidean symmetry in the spatial directions $[1,70]$. Choosing a convenient gauge for the radial coordinate, they can be written as

$$
\mathrm{d} s^{2}=g_{m n}^{(0)} \mathrm{d} x^{m} \mathrm{~d} x^{n}=e^{2 A(u)}\left[-f(u) \mathrm{d} t^{2}+\mathrm{d} \underline{x}^{2}\right]+\frac{L^{2}}{4 u^{2} f(u)} \mathrm{d} u^{2},
$$

\footnotetext{
${ }^{6}$ We restrict ourselves to operators of dimension $\Delta=3$ because the holographic renormalisation has already been done for this class of holographic RG flows, see appendix $\mathrm{C}$ of ref. [13] for details.
} 
where $f(u)$ has a simple zero at the horizon and the Hawking temperature $T$ and entropy density $s$ are given by

$$
T=\left.\frac{-f^{\prime}(u) e^{A(u)}}{2 \pi L}\right|_{u=1}, \quad s=\left.\frac{e^{3 A(u)}}{4 G_{N}}\right|_{u=1} .
$$

The residual scaling symmetry, inherited from the UV CFT, can be used to set the horizon position to $u=1$, effectively expressing all dimensionful quantities in units of temperature. The equations of motion that follow from the action (4.24) take the following form in the gauge (4.27):

$$
\begin{aligned}
\phi^{\prime \prime}+\left(4 A^{\prime}+\frac{1}{u}\right) \phi^{\prime}+\frac{f^{\prime}}{f} \phi^{\prime}-\frac{L^{2}}{4 u^{2} f}\left(\frac{\mathrm{d} V}{\mathrm{~d} \phi}\right) & =0, \\
A^{\prime \prime}+\frac{1}{u} A^{\prime}+\frac{1}{6}\left(\phi^{\prime}\right)^{2} & =0, \\
f^{\prime \prime}+\left(4 A^{\prime}+\frac{1}{u}\right) f^{\prime} & =0, \\
6 A^{\prime} f^{\prime}+f\left[24\left(A^{\prime}\right)^{2}-\left(\phi^{\prime}\right)^{2}\right]+\frac{L^{2}}{2 u^{2}} V & =0,
\end{aligned}
$$

where primes denote derivatives with respect to $u$. The system is partly redundant in the sense that the constraint $(4.29 \mathrm{~d})$ and its derivative are algebraically given in terms of the other three equations:

$$
\left(\frac{\mathrm{d}}{\mathrm{d} u}+\frac{2}{u}\right)(4.29 \mathrm{~d})=-2 f \frac{\mathrm{d} \phi}{\mathrm{d} u}(4.29 \mathrm{a})+\left(48 f A^{\prime}+6 f^{\prime}\right)(4.29 \mathrm{~b})+6 A^{\prime}(4.29 \mathrm{c}) .
$$

For vanishing scalar, $\phi=0$, eqs. (4.29) are solved by the $A d S_{5}$ black-brane background:

$$
A(u)=\frac{1}{2} \log \left[\frac{(\pi T L)^{2}}{u}\right], \quad f(u)=1-u^{2} .
$$

\subsubsection{Equations for metric perturbations}

We will now present the dual gravity description of the (field-theory) metric perturbations we discussed in section 4.2. Generally, the external metric $g_{(0) \mu \nu}$ of QFTs with a holographic dual prescribes the value of the dual dynamical bulk metric $g_{m n}$ at the AdS boundary [3]. Perturbations $h_{\mu \nu}$ of the field-theory metric source changes in the bulk metric, which in turn encode the response of the QFT stress tensor $\left\langle T^{\mu \nu}\right\rangle[53,62]$. 
Denoting the field-theory directions by $x^{\mu}=(t, x, y, z)$ and maintaining a radial gauge $g_{u \mu}=0$, we will write the perturbed bulk metric as

$$
\mathrm{d} s^{2}=g_{m n} \mathrm{~d} x^{\mu} \mathrm{d} x^{n}=g_{m n}^{(0)} \mathrm{d} x^{m} \mathrm{~d} x^{n}+\epsilon g_{\mu \nu}^{(1)} \mathrm{d} x^{\mu} \mathrm{d} x^{\nu}+\epsilon^{2} g_{\mu \nu}^{(2)} \mathrm{d} x^{\mu} \mathrm{d} x^{\nu}+\mathcal{O}\left(\epsilon^{3}\right)
$$

where $g_{m n}^{(0)}$ is the background metric $(4.27), \epsilon g_{\mu \nu}^{(1)}$ contains the sourced metric perturbations at the boundary, and $\epsilon^{2} g_{\mu \nu}^{(2)}$ describes their $\mathcal{O}\left(\epsilon^{2}\right)$ backreaction on the bulk. The form of $g_{\mu \nu}^{(1)}$ and $g_{\mu \nu}^{(2)}$ varies depending on which of the three metric perturbations (4.14), (4.16) and (4.18) we turn on. However, none of these transverse-vector perturbations source linear fluctuations of the scalar field at $\mathcal{O}(\epsilon)$, and fluctuations of $\phi$ at $\mathcal{O}\left(\epsilon^{2}\right)$ do not affect $g_{x y}^{(2)} \cdot 7$

Turning on $\left\{h_{x z}(t), h_{y z}(t)\right\}$ : The metric perturbation (4.14) corresponds to

$$
\begin{aligned}
\frac{1}{2} g_{\mu \nu}^{(1)} \mathrm{d} x^{\mu} \mathrm{d} x^{\nu}=e^{2 A(u)} & {\left[H_{x z}^{(b)} e^{-i q_{0} t} H^{(1 t)}\left(u, q_{0}\right) \mathrm{d} x \mathrm{~d} z\right.} \\
& \left.+H_{y z}^{(b)} e^{-i p_{0} t} H^{(1 t)}\left(u, p_{0}\right) \mathrm{d} y \mathrm{~d} z\right]
\end{aligned}
$$

where Einstein's equations at order $\mathcal{O}(\epsilon)$ reduce to a frequency-dependent equation for the function $H^{(1 t)}$,

$$
H^{(1 t) \prime \prime}(u, \omega)+\left(\frac{1}{u}+4 A^{\prime}+\frac{f^{\prime}}{f}\right) H^{(1 t) \prime}(u, \omega)+\frac{e^{-2 A} L^{2} \omega^{2}}{4 u^{2} f^{2}} H^{(1 t)}(u, \omega)=0,
$$

normalised to $H^{(1 t)}(u=0)=1$ at the boundary. The $x y$-component of the resulting backreaction at $\mathcal{O}\left(\epsilon^{2}\right)$ is conveniently parametrised as

$$
g_{x y}^{(2)}=e^{2 A(u)} H_{x z}^{(b)} H_{y z}^{(b)} e^{-i p_{0} t-i q_{0} t} H^{(2 t t)}\left(u, q_{0}, p_{0}\right)
$$

so that the $x y$-component of Einstein's equations at $\mathcal{O}\left(\epsilon^{2}\right)$ becomes

$$
\begin{aligned}
& H^{(2 t t) \prime \prime}(u)+\left(\frac{1}{u}+4 A^{\prime}+\frac{f^{\prime}}{f}\right) H^{(2 t t) \prime}(u)+\frac{e^{-2 A} L^{2}\left(q_{0}+p_{0}\right)^{2}}{4 u^{2} f^{2}} H^{(2 t t)}\left(u, q_{0}, p_{0}\right) \\
& =\frac{e^{-2 A} L^{2} q_{0} p_{0}}{4 u^{2} f^{2}} H^{(1 t)}\left(u, q_{0}\right) H^{(1 t)}\left(u, p_{0}\right)+H^{(1 t) \prime}\left(u, q_{0}\right) H^{(1 t) \prime}\left(u, p_{0}\right) .
\end{aligned}
$$

The fluctuation $H^{(2 t t)}$ is not sourced by an explicit deformation of the boundary metric, $H^{(2 t t)}(u=0)=0$, but only by the backreaction of the first-order perturbations $g_{\mu \nu}^{(1)}$.

\footnotetext{
${ }^{7}$ Metric perturbations in the scalar sound channel, on the other hand, would source linear fluctuations of $\phi$. It is for this technical reason that we restrict ourselves to perturbations in the transverse shear channel. The drawback is that this only gives us access to five independent combinations of transport coefficients, eq. (4.2), as explained in section 4.2.1.
} 
Turning on $\left\{h_{t x}(z), h_{t y}(z)\right\}$ : The metric perturbation (4.16) corresponds to

$$
\frac{1}{2} g_{\mu \nu}^{(1)} \mathrm{d} x^{\mu} \mathrm{d} x^{\nu}=e^{2 A(u)}\left[H_{t x}^{(b)} e^{i q_{z} z} H^{(1 z)}\left(u, q_{z}\right) \mathrm{d} t \mathrm{~d} x+H_{t y}^{(b)} e^{i p_{z} z} H^{(1 z)}\left(u, p_{z}\right) \mathrm{d} t \mathrm{~d} y\right]
$$

where Einstein's equations at order $\mathcal{O}(\epsilon)$ reduce to a momentum-dependent equation for the function $H^{(1 z)}$,

$$
H^{(1 z) \prime \prime}(u, \omega)+\left(\frac{1}{u}+4 A^{\prime}\right) H^{(1 z) \prime}(u, \omega)-\frac{e^{-2 A} L^{2} \omega^{2}}{4 u^{2} f} H^{(1 z)}(u, \omega)=0
$$

normalised to $H^{(1 z)}(u=0)=1$ at the boundary. The $x y$-component of the resulting backreaction at $\mathcal{O}\left(\epsilon^{2}\right)$ is conveniently parametrised as

$$
g_{x y}^{(2)}=e^{2 A(u)} H_{t x}^{(b)} H_{t y}^{(b)} e^{i q_{z} z+i p_{z} z} H^{(2 z z)}\left(u, q_{z}, p_{z}\right)
$$

so that the $x y$-component of Einstein's equations at $\mathcal{O}\left(\epsilon^{2}\right)$ becomes

$$
\begin{aligned}
& H^{(2 z z) \prime \prime}(u)+\left(\frac{1}{u}+4 A^{\prime}+\frac{f^{\prime}}{f}\right) H^{(2 z z) \prime}(u)-\frac{e^{-2 A} L^{2}\left(q_{z}+p_{z}\right)^{2}}{4 u^{2} f} H^{(2 z z)}\left(u, q_{z}, p_{z}\right) \\
& =\frac{e^{-2 A} L^{2} q_{z} p_{z}}{4 u^{2} f^{2}} H^{(1 z)}\left(u, q_{z}\right) H^{(1 z)}\left(u, p_{z}\right)-\frac{1}{f} H^{(1 t) \prime}\left(u, q_{z}\right) H^{(1 z) \prime}\left(u, p_{z}\right) .
\end{aligned}
$$

The fluctuation $H^{(2 z z)}$ is not sourced by an explicit deformation of the boundary metric, $H^{(2 z z)}(u=0)=0$.

Turning on $\left\{h_{t y}(z), h_{x z}(t)\right\}$ : The metric perturbation (4.18) corresponds to

$$
\begin{aligned}
& \frac{1}{2} g_{\mu \nu}^{(1)} \mathrm{d} x^{\mu} \mathrm{d} x^{\nu} \\
& =e^{2 A(u)}\left[H_{t y}^{(b)} e^{i p_{z} z} H^{(1 z)}\left(u, p_{z}\right) \mathrm{d} t \mathrm{~d} y+H_{x z}^{(b)} e^{-i q_{0} t} H^{(1 t)}\left(u, q_{0}\right) \mathrm{d} x \mathrm{~d} z\right]
\end{aligned}
$$

where Einstein's equations at order $\mathcal{O}(\epsilon)$ reduce to eqs. (4.34) and (4.38) for $H^{(1 t)}$ and $H^{(1 z)}$ respectively. ${ }^{8}$ The $x y$-component of the resulting backreaction at $\mathcal{O}\left(\epsilon^{2}\right)$ is conveniently parametrised as

$$
g_{x y}^{(2)}=e^{2 A(u)} H_{t y}^{(b)} H_{x z}^{(b)} e^{-i q_{0} t+i p_{z} z} H^{(2 t z)}\left(u, q_{0}, p_{z}\right)
$$

${ }^{8}$ Moreover, $H^{(1 t)}$ and $H^{(1 z)}$ are again normalised to 1 at the boundary and, as we will discuss in section 4.4.2, are subject to the same boundary conditions at the horizon as they are in cases (4.33) and (4.37). Hence $H^{(1 t)}$ and $H^{(1 z)}$ here are indeed the same as in perturbations (4.33) and (4.37). 
so that the $x y$-component of Einstein's equations at $\mathcal{O}\left(\epsilon^{2}\right)$ becomes

$$
\begin{aligned}
& H^{(2 t z) \prime \prime}(u)+\left(\frac{1}{u}+4 A^{\prime}+\frac{f^{\prime}}{f}\right) H^{(2 t z) \prime}(u)-\frac{e^{-2 A} L^{2}\left(-q_{0}^{2}+f p_{z}^{2}\right)}{4 u^{2} f^{2}} H^{(2 t z)}\left(u, q_{0}, p_{z}\right) \\
& =\frac{e^{-2 A} L^{2} q_{0} p_{z}}{4 u^{2} f^{2}} H^{(1 t)}\left(u, q_{0}\right) H^{(1 z)}\left(u, p_{z}\right)
\end{aligned}
$$

The fluctuation $H^{(2 t z)}$ is not sourced by an explicit deformation of the boundary metric, $H^{(2 t z)}(u=0)=0$.

\subsection{Solving Einstein's equations}

In the previous section we introduced a class of non-conformal holographic models and presented the corresponding equations of motion for static black-brane backgrounds (subsection 4.3.1) and for metric fluctuations around these backgrounds (subsection 4.3.2), sourced by perturbations (4.14), (4.16), (4.18) of the field-theory metric. Our next goal is to determine the response of the field-theory stress tensor $\left\langle T^{\mu \nu}\right\rangle$ to these perturbations. To this end, we need to find solutions to the bulk equations of motion that satisfy the appropriate boundary conditions at the AdS boundary and at the horizon. The stress tensor is then encoded in the near-boundary expansion of the bulk metric $[53,163]$.

In this section we want to see how far we can get solving for fluctuations of the bulk metric around an arbitrary background solution without specifying the scalar potential (4.25) beyond the mass term. The results of this section therefore apply to all holographic RG flows triggered by a scalar operator of dimension $\Delta=3$, at any value of the temperature. We begin by writing down the near-horizon and near-boundary expansion of a general background solution in subsection 4.4.1. The former is needed to identify which boundary conditions to impose on metric fluctuations at the horizon while the latter is necessary for the computation of $\left\langle T^{\mu \nu}\right\rangle$. In subsection 4.4.2 we turn to fluctuations of the bulk metric: we impose the appropriate boundary conditions, perform the hydro expansion up to second order in momenta, determine local solutions near horizon and boundary, and try to find global solutions connecting the two. We 
managed to solve for all but five of the hydro metric fluctuations analytically, and we found integral expressions for another four. ${ }^{9}$

We appreciate that this is a rather technical section. For practical purposes, integrals (4.65), which yield the sub-leading boundary modes for four hydro metric fluctuations, make up the central result we will refer to in subsequent sections.

\subsubsection{Local analysis of background solutions}

Near-horizon expansion: The fields $A(u), f(u)$ and $\phi(u)$ satisfy a system of three second-order equations (4.29a)-(4.29c) and one first-order equation (4.29d). The coefficients in the local series solution around the horizon are not constrained by $(4.29 \mathrm{~d})$ thanks to the redundancy (4.30). The general local near-horizon solution therefore contains six integration constants. Demanding that $A$ and $\phi$ be regular at the horizon and that the horizon position be at $u=1$ amounts to three boundary conditions. The near-horizon expansions of $A, f$, and $\phi$ thus depend on three near-horizon modes $\left\{A_{H}, f_{H}, \phi_{H}\right\}$ and assume the form

$$
\begin{aligned}
A(u) & =A_{H}+\sum_{k \geq 1} b_{k}^{A}(1-u)^{k}, \\
f(u) & =(1-u)\left[f_{H}+\sum_{k \geq 1} b_{k}^{f}(1-u)^{k}\right], \\
\phi(u) & =\phi_{H}+\frac{L^{2} V^{\prime}\left(\phi_{H}\right)}{4 f_{H}}(1-u)+\sum_{k \geq 2} b_{k}^{\phi}(1-u)^{k},
\end{aligned}
$$

with series coefficients fully determined by the near-horizon modes and the given potential.

Near-boundary expansion: The general local near-boundary solution to (4.29) contains six integration constants, two of which we fix by demanding that the spacetime be asymptotically $A d S_{5}$,

$$
A(u)=-\frac{1}{2} \log (u)+\mathcal{O}\left(u^{0}\right), \quad f(u)=1+\mathcal{O}(u) .
$$

\footnotetext{
${ }^{9}$ Curiously, it turns out that the one hydro metric fluctuation for which we did not find a solution cancels out in the expression for $\left\langle T^{\mu \nu}\right\rangle$ presented in subsection 4.5.1.
} 
The redundancy (4.30) implies that at each order in $u$, the four equations of motion (4.29) only provide three independent algebraic equations which determine the corresponding series coefficients of $A, f$ and $\phi$. When we reach the order of one of the remaining four integration constants (the sub-leading modes of $A$ and $f$, and the two modes of the scalar $\phi$ ), then the three algebraic equations fail to fix these (free) parameters. Instead, either only two of the algebraic equations are independent at this order, or one of them restricts the form of potentials $V$, eq. (4.25), compatible with the requirement of asymptotically $A d S_{5}$.

One finds that near-boundary solutions take the form

$$
\begin{aligned}
& A(u)=\frac{1}{2} \log \left(\frac{A_{b}}{u}\right)-\frac{\phi_{L}^{2}}{24} u+\sum_{k \geq 2} c_{k}^{A} u^{k}, \\
& f(u)=1+f_{b} u^{2}+u^{2} \sum_{k \geq 1} c_{k}^{f} u^{k}, \\
& \phi(u)=\phi_{L} \sqrt{u}+\phi_{S L} u^{3 / 2}+u^{3 / 2} \sum_{k \geq 1} c_{k}^{\phi} u^{k},
\end{aligned}
$$

with series coefficients fully determined by $V$ and the four boundary modes $A_{b}, f_{b}, \phi_{L}$, $\phi_{S L}$, and that the potential must satisfy ${ }^{10}$

$$
4 ! \frac{\mathrm{d}^{4} V}{\mathrm{~d} \phi^{4}}=-\frac{1}{12 L^{2}}
$$

As the local near-boundary solution cannot depend on more than four free parameters, the three independent equations coming from (4.29) must succeed in fixing the three corresponding series coefficients of $A, f$, and $\phi$ to all orders beyond the fields' subleading modes. In particular, no further constraints on the potential $V(\phi)$ can arise.

\subsubsection{Solutions for metric perturbations}

Boundary conditions: In order to compute the retarded response of the stress tensor, time-dependent perturbations of the bulk metric need to represent incoming waves at the horizon $[47,73]$ while static perturbations are simply required to be regular at

\footnotetext{
${ }^{10}$ Note that superpotentials $W$ which lead to $L^{2} V=-12-(3 / 2) \phi^{2}+\mathcal{O}\left(\phi^{4}\right)$, i.e. $L W=-(3 / 2)-$ $\phi^{2} / 8+\mathcal{O}\left(\phi^{4}\right)$, automatically ensure that condition (4.47) is satisfied. We believe that condition (4.47) was overlooked in ref. [142].
} 
the horizon. Let us define momenta in Fraktur as the dimensionless combination

$$
\mathfrak{w} \equiv \frac{L \omega}{2 f_{H} e^{A_{H}}}=\frac{\omega}{4 \pi T},
$$

where we used expression (4.28) for the temperature. The incoming-wave solution to eq. (4.34) and the regular solution to eq. (4.38) take the form

$$
\begin{aligned}
& H^{(1 t)}(u, \omega)=(1-u)^{-i \mathfrak{w}} K^{(1 t)}(u, \omega) \\
& H^{(1 z)}(u, \omega)=(1-u) K^{(1 z)}(u, \omega)
\end{aligned}
$$

where $K^{(1 \alpha)}, \alpha \in\{t, z\}$, are analytic at the horizon and normalised to 1 at the boundary. The first-order perturbations dictate the form of the second-order fluctuations they source. Investigating eqs. (4.36), (4.40), (4.43) shows that

$$
\begin{aligned}
& H^{(2 t t)}\left(u, q_{0}, p_{0}\right)=(1-u)^{-i \mathfrak{q}_{0}-i \mathfrak{p}_{0}} K^{(2 t t)}\left(u, q_{0}, p_{0}\right), \\
& H^{(2 z z)}\left(u, q_{z}, p_{z}\right)=K^{(2 z z)}\left(u, q_{z}, p_{z}\right) \\
& H^{(2 t z)}\left(u, q_{0}, p_{z}\right)=(1-u)^{-i \mathfrak{q}_{0}} K^{(2 t z)}\left(u, q_{0}, p_{z}\right)
\end{aligned}
$$

where $K^{(2 \beta)}, \beta \in\{t t, z z, t z\}$, are analytic at the horizon and vanish at the boundary.

Hydrodynamic gradient expansion: To match the holographic result for $\left\langle T^{x y}\right\rangle$ with the general hydro form discussed in section 4.2.1, we need to turn on sources that admit an expansion in small gradients/momenta. We therefore expand the metric fluctuations as

$$
\begin{aligned}
& K^{(1 \alpha)}(u, \omega)=K_{0}^{(1 \alpha)}(u)+K_{1}^{(1 \alpha)}(u) \omega+K_{2}^{(1 \alpha)}(u) \omega^{2}+\mathcal{O}\left(\mathfrak{w}^{3}\right), \quad \alpha \in\{t, z\}, \\
& K^{(2 \beta)}(u, q, p)=K_{(0,0)}^{(2 \beta)}(u)+\left[K_{(1,0)}^{(2 \beta)}(u) q+K_{(0,1)}^{(2 \beta)}(u) p\right] \\
& \quad+\left[K_{(2,0)}^{(2 \beta)}(u) q^{2}+K_{(1,1)}^{(2 \beta)}(u) q p+K_{(0,2)}^{(2 \beta)}(u) p^{2}\right]+(\mathcal{O}(\mathfrak{q}, \mathfrak{p}))^{3}, \quad \beta \in\{t t, z z, t z\} .
\end{aligned}
$$

To simplify the discussion we shall use $a$ to label the metric perturbations and $j$ to denote the order in the hydro expansion, i.e. $j \in\{0,1, \ldots\}$ for $a \in\{1 t, 1 z\}$ and $j \in$ $\{(0,0),(1,0),(0,1), \ldots\}$ for $a \in\{2 t t, 2 z z, 2 t z\}$.

Expanding the equations of motion (4.34), (4.38), (4.36), (4.40), (4.43) in momenta reveals that not all of the $K_{j}^{(a)}$ are independent. Firstly, $K_{(1,0)}^{(2 t t)}$ and $K_{(0,1)}^{(2 t t)}$ satisfy the 
same equation and boundary conditions and are thus identical. As a consequence the same holds for $K_{(2,0)}^{(2 t t)}$ and $K_{(0,2)}^{(2 t t)}$. Furthermore, $K_{1}^{(1 z)}$ satisfies a linear and homogeneous equation. The solution that is regular at the horizon and vanishes at the boundary is identically zero. The same is then true for $K_{(1,0)}^{(2 z z)}, K_{(0,1)}^{(2 z z)}, K_{(0,0)}^{(2 t z)}, K_{(1,0)}^{(2 t z)}, K_{(0,1)}^{(2 t z)}, K_{(2,0)}^{(2 t z)}$, and $K_{(0,2)}^{(2 t z)}$ which all vanish identically. Finally, $K_{(2,0)}^{(2 z z)}$ and $K_{(0,2)}^{(2 z z)}$ are also subject to the same equation and boundary conditions and are therefore identical. These identities essentially follow from simple symmetry properties and the fact that the considered boundary metric perturbations (4.14), (4.16), (4.18) do not source all 24 possible bulk fluctuations $K_{j}^{(a)}$.

Local solutions: The $K_{j}^{(a)}$ have been defined to be analytic at the horizon where the local solution thus depends on a single near-horizon mode $Z_{j}^{(a)}$ :

$$
K_{j}^{(a)}(u)=Z_{j}^{(a)}+\sum_{s \geq 1} \lambda_{j, s}^{(a)}(1-u)^{s}
$$

The equations of motion (4.34), (4.38), (4.36), (4.40), (4.43) also show that solutions can be expanded near the boundary as

$$
K_{j}^{(a)}=X_{j}^{(a)}+k_{j, 1}^{(a)} u+Y_{j}^{(a)} u^{2}+\sum_{s \geq 3} k_{j, s}^{(a)} u^{s}+\log u \sum_{s \geq 2} l_{j, s}^{(a)} u^{2},
$$

with leading mode $X_{j}^{(a)}$ and sub-leading mode $Y_{j}^{(a)}$. The boundary conditions discussed around eqs. (4.49) and (4.50) amount to

$$
\begin{array}{lr}
X_{0}^{(1 \alpha)}=1, X_{j \geq 1}^{(1 \alpha)}=0, & \alpha \in\{t, z\}, \\
X_{j}^{(2 \beta)}=0, & \beta \in\{t t, z z, t z\} .
\end{array}
$$

Global solutions: Some of the $K_{j}^{(a)}(u)$ can be solved for analytically. Using the constraint $(4.29 \mathrm{~d})$ on the background, $\partial_{u} K_{0}^{(1 t)}$ and $\partial_{u} K_{(0,0)}^{(2 t t)}$ are found to satisfy homogeneous linear first-order equations. The unique regular solutions that are normalised to 1 and 0 at the boundary respectively are

$$
K_{0}^{(1 t)}=1
$$


This in turn renders the equations satisfied by $K_{(1,0)}^{(2 t t)}$ and $K_{(2,0)}^{(2 t t)}$ homogeneous. The unique regular solutions that vanish at the boundary are identically zero,

$$
\begin{aligned}
& K_{(1,0)}^{(2 t t)}=0, \\
& K_{(2,0)}^{(2 t t)}=0 .
\end{aligned}
$$

Likewise, employing the constraint $(4.29 \mathrm{~d})$ and replacing $A^{\prime}(u)$ using eq. (4.29c) one can successively solve for $K_{0}^{(1 z)}, K_{(0,0)}^{(2 z z)}$, and $K_{1}^{(1 t)}$ :

$$
\begin{aligned}
& K_{0}^{(1 z)}=\frac{f(u)}{1-u}, \quad K_{(0,0)}^{(2 z z)}=1-f(u), \\
& K_{1}^{(1 t)}=-\frac{i}{4 \pi T} \log \left(\frac{f(u)}{1-u}\right) .
\end{aligned}
$$

Finally, comparing the corresponding equations of motion reveals that

$$
K_{(2,0)}^{(2 z z)}=-(1-u) K_{2}^{(1 z)}+K_{(1,1)}^{(2 t z)}
$$

This leaves us with five of the initial 24 functions $K_{j}^{(a)}$ still undetermined:

$$
K_{2}^{(1 z)}, K_{2}^{(1 t)}, K_{(1,1)}^{(2 t t)}, K_{(1,1)}^{(2 z z)}, K_{(1,1)}^{(2 t z)}
$$

We did not manage to solve for $K_{2}^{(1 z)}$, but we can do a little better with the other four. Owing to the residual gauge symmetry at second order $\mathcal{O}\left(\epsilon^{2}\right)$ in metric perturbations, their equations of motion only depend on the functions' derivatives and take the form

$$
\frac{\mathrm{d}}{\mathrm{d} u}\left[u f(u) e^{4 A(u)} \frac{\mathrm{d}}{\mathrm{d} u} K_{j}^{(a)}(u)\right]=u f(u) e^{4 A(u)} \Upsilon_{j}^{(a)}(u),
$$

where

$$
K_{j}^{(a)} \in\left\{K_{2}^{(1 t)}, K_{(1,1)}^{(2 t t)}, K_{(1,1)}^{(2 z z)}, K_{(1,1)}^{(2 t z)}\right\}
$$

The explicit expressions for the $\Upsilon_{j}^{(a)}$, which only depend on the background, are written in eq. (B.4) in appendix B.2. Using that the four $K_{j}^{(a)}$ at hand are zero at the boundary and that the regularity condition (4.52) implies that the square bracket in eq. (4.62) vanishes at the horizon, we can formally integrate eq. (4.62) to

$$
K_{j}^{(a)}(u)=\int_{0}^{u} \mathrm{~d} v \frac{1}{v f(v) e^{4 A(v)}} \int_{1}^{v} \mathrm{~d} w w f(w) e^{4 A(w)} \Upsilon_{j}^{(a)}(w) .
$$


Expanding the solution near the boundary (see appendix B.2 for details) we can read off the corresponding sub-leading modes,

$$
\begin{aligned}
Y_{j}^{(a)}= & \frac{1}{8 \pi^{2} T^{2}}\left(\frac{f_{H}^{2} e^{2 A_{H}}}{4 A_{b}}\right)\left\{ \pm\left(1-\frac{\phi_{L}^{2}}{8}\right)\right. \\
& \left.-\int_{0}^{1} \mathrm{~d} w\left[4 w f(w) e^{4 A(w)} \frac{\Upsilon_{j}^{(a)}(w)}{A_{b} L^{2}} \mp \frac{1}{w^{2}}\left(1-\frac{\phi_{L}^{2}}{12} w\right)\right]\right\},
\end{aligned}
$$

where the upper signs refer to $K_{j}^{(a)} \in\left\{K_{(1,1)}^{(2 t t)}, K_{(1,1)}^{(2 z z)}, K_{(1,1)}^{(2 t z)}\right\}$ and the lower signs to $K_{j}^{(a)}=K_{2}^{(1 t)}$. In the conformal case $\phi=0$, eq. (4.31), the integrals can be performed analytically, resulting in

$$
\left(Y_{2}^{(1 t)}, Y_{(1,1)}^{(2 t t)}, Y_{(1,1)}^{(2 z z)}, Y_{(1,1)}^{(2 t z)}\right) \stackrel{\phi \rightarrow 0}{\longrightarrow}\left(\frac{-5+4 \log 2}{32 \pi^{2} T^{2}}, \frac{1-\log 2}{8 \pi^{2} T^{2}}, 0, \frac{1}{8 \pi^{2} T^{2}}\right)
$$

\subsection{Analytical results for second-order transport}

This section contains our analytical results for second-order transport in the class of non-conformal holographic models introduced in section 4.3. We provide explicit formulae for the five second-order coefficients (4.2) in subsection (4.5.1). They apply to all holographic RG flows triggered by a scalar operator of dimension $\Delta=3$, at any value of the temperature. Notably, we find that the particular combination $\tilde{H} \equiv 2 \eta \tau_{\pi}-2\left(\kappa-\kappa^{*}\right)-\lambda_{2}$ vanishes identically in this class of models. In subsection (4.5.2) we prove that the identity $H=2 \eta \tau_{\pi}-4 \lambda_{1}-\lambda_{2}=0$, which is universally satisfied by infinitely strongly coupled holographic fluids with conformal symmetry [14], still holds when taking into account leading non-conformal corrections to the transport coefficients.

\subsubsection{Formulae for transport coefficients}

To compute the transport coefficients we need to match the effective hydro result for the field-theory stress tensor $\left\langle T^{\mu \nu}\right\rangle$ with the corresponding holographic result. After a suitable renormalisation procedure, the latter can be read off from the near-boundary expansion of the dual bulk metric $[53,163]$. Details on this calculation can be found 
in appendix $\mathrm{C}$ of ref. [13]. Once we apply the solutions for the bulk metric perturbations from the previous section, the result will depend on the near-boundary modes $\left\{A_{b}, f_{b}, \phi_{L}, \phi_{S L}\right\}$ of the background, on the temperature $T$ which sets the unit of momenta, and on the sub-leading modes $\left\{Y_{2}^{(1 z)}, Y_{2}^{(1 t)}, Y_{(1,1)}^{(2 t t)}, Y_{(1,1)}^{(2 z z)}, Y_{(1,1)}^{(2 t z)}\right\}$ of the metric perturbations (4.61) without closed-form solutions. We can somewhat simplify the result by making use of the fact that $f^{\prime}(u)$ satisfies the first-order equation $(4.29 \mathrm{c})$. Imposing the requirement of asymptotically $A d S_{5}$, eq. (4.46), it can be integrated to give

$$
f^{\prime}(u)=2 f_{b} \frac{A_{b}^{2} e^{-4 A(u)}}{u}
$$

Evaluating this at the horizon and using expressions (4.28) for temperature $T$ and entropy density $s$ reveals that

$$
A_{b}^{2}=-\left(\frac{4 \pi G_{N} L}{f_{b}}\right) s T .
$$

Finally, we can re-express the leading- and sub-leading modes $\phi_{L}$ and $\phi_{S L}$ of the scalar in terms of source $\Lambda$ and expectation value $\langle O\rangle$ of the scalar operator, employing the following relations from the holographic renormalisation (see appendix C in ref. [13]):

$$
\Lambda=\frac{\sqrt{A_{b}}}{L} \phi_{L}, \quad\langle O\rangle=\frac{L^{3}}{8 \pi G_{N}}\left(\frac{A_{b}}{L^{2}}\right)^{3 / 2} \phi_{S L}+\mathcal{O}\left(\epsilon^{2}\right) .
$$

We are ready at last to present the holographic result for $\left\langle T^{\mu \nu}\right\rangle$ in units of the field-theory quantities $T, s, \Lambda$, and $\langle O\rangle$. The background stress tensor at $\mathcal{O}\left(\epsilon^{0}\right)$ takes the ideal-fluid form

$$
\bar{T}^{\mu \nu}=\left(\begin{array}{cccc}
\bar{\epsilon} & & & \\
& \bar{p} & & \\
& & \bar{p} & \\
& & & \bar{p}
\end{array}\right)
$$

with energy density

$$
\bar{\epsilon}=\frac{3}{4} s T-\frac{1}{4} \Lambda\langle O\rangle
$$

and pressure $^{11}$

$$
\bar{p}=\frac{1}{4} s T+\frac{1}{4} \Lambda\langle O\rangle
$$

\footnotetext{
${ }^{11}$ In the conformal case $\phi=0$, eq. (4.31), this reduces to $\bar{\epsilon}=3 \bar{p}=\frac{3 \pi^{3} L^{3}}{16 G_{N}} T^{4}$ or $\bar{\epsilon}=3 \bar{p}=\frac{3 \pi^{2}}{8} N^{2} T^{4}$ for $\mathcal{N}=4$ specifically [164].
} 
For each of the perturbations (4.14), (4.16), (4.18), the leading response of the transverse-tensor component $\left\langle T^{x y}\right\rangle$ occurs at order $\mathcal{O}\left(\epsilon^{2}\right)$ and indeed takes the expected hydro form (4.15), (4.17), (4.19). Owing to relation (4.68), the shear viscosity assumes its universal value in units of $s[7,67,94,121-126]$

$$
\eta=\frac{1}{4 \pi} s
$$

while the second-order coefficients are given by the following expressions:

$$
\begin{aligned}
\kappa & =-\frac{2}{f_{b}} Y_{(1,1)}^{(2 t z)} s T \\
\eta \tau_{\pi}+\kappa^{*} & =\frac{1}{f_{b}}\left(\frac{1}{32 \pi^{2} T^{2}}+Y_{2}^{(1 t)}-Y_{(1,1)}^{(2 t z)}\right) s T \\
\lambda_{1}+\frac{\kappa^{*}}{2} & =\frac{1}{f_{b}}\left(\frac{1}{32 \pi^{2} T^{2}}+Y_{2}^{(1 t)}-Y_{(1,1)}^{(2 t z)}+Y_{(1,1)}^{(2 t t)}\right) s T, \\
\lambda_{2} & =\frac{2}{f_{b}}\left(\frac{1}{32 \pi^{2} T^{2}}+Y_{2}^{(1 t)}+Y_{(1,1)}^{(2 t z)}\right) s T, \\
\lambda_{3}-2 \kappa^{*} & =\frac{4}{f_{b}} Y_{(1,1)}^{(2 z z)} s T .
\end{aligned}
$$

The solutions for the $Y_{j}^{(a)}$ that appear in eq. (4.74) are stated in eq. (4.65). The fact that $\left\langle T^{x y}\right\rangle$ assumes the form dictated by hydrodynamics is a non-trivial check on our computations. In particular, the correct result for $\eta$ relies on identities (4.56) and (4.59), while identities (4.55) and (4.58) are essential to ensuring that the secondorder responses (4.15) and (4.17) both reproduce the same pressure as the background stress tensor (4.70) does.

We further note that the dependence of the second-order coefficients (4.74) on $Y_{2}^{(1 z)}$ cancels out as a consequence of relation (4.60). The five a-priori independent combinations (4.74) hence only depend on the four sub-leading modes $Y_{2}^{(1 t)}, Y_{(1,1)}^{(2 t t)}, Y_{(1,1)}^{(2 z z)}, Y_{(1,1)}^{(2 t z)}$ given by eq. (4.65). In particular, there exists one linear combination of second-order coefficients that is independent of the $Y_{j}^{(a)}$ :

$$
\tilde{H} \equiv 2 \eta \tau_{\pi}-2\left(\kappa-\kappa^{*}\right)-\lambda_{2}=0
$$

This identity does not depend on a particular background solution and therefore holds for all holographic RG flows triggered by a scalar operator of dimension $\Delta=3$, at 
any value of the temperature. We would also like to emphasise that it crucially relies on the global solutions (4.55)-(4.60) we found for some of the metric perturbations. Accordingly, identity (4.75) cannot simply follow from Ward identities which rely only on the local near-boundary solutions $[53,60]$. To our knowledge, the only holographic theories in which all transport coefficients entering $\tilde{H}$ have been computed are planar $\mathcal{N}=4$ (at infinite 't Hooft coupling $[10,11,43]$ as well as including leading finite coupling corrections [136, 165-170]) and the non-conformal Chamblin-Reall background [140]. In both cases $\tilde{H}$ vanishes in the infinite coupling limit, but it becomes non-zero when taking into account finite coupling corrections in $\mathcal{N}=4$.

We conclude this subsection by illustrating the usability of eqs. (4.74). They allow for the straightforward computation of the second-order coefficients (4.2) for any given background solution $A(u), f(u), \phi(u)$ of Einstein's equations (4.29) coupled to a scalar with potential $V$, eq. (4.25): simply extract horizon and boundary modes of the background according to eqs. (4.44) and (4.46), perform integrals (4.65) over the background to compute the four $Y_{j}^{(a)}$, and plug the result into eqs. (4.74). For instance, the values (4.66) of the $Y_{j}^{(a)}$ in the conformal case, eq. (4.31), readily reproduce the known results for all second-order transport coefficients in conformal holographic fluids $\left(\kappa^{*}=0\right)[10,11]$,

$$
\left\{\kappa, \eta \tau_{\pi}, \lambda_{1}, \lambda_{2}, \lambda_{3}\right\}=\left(\frac{s}{8 \pi^{2} T}\right)\{2,2-\log 2,1,-2 \log 2,0\},
$$

where for $\mathcal{N}=4$ specifically $s / T=\pi^{2} N^{2} T^{2} / 2$.

\subsubsection{Proof that $H=0$ to leading order away from conformality}

We will now prove that, to leading order in the deviation from conformality, $H=$ $2 \eta \tau_{\pi}-4 \lambda_{1}-\lambda_{2}$ vanishes for any uncharged holographic $C F T_{4}$ deformed by a relevant scalar operator of dimension $\Delta=3$. From eq. (4.74), $H$ takes the form

$$
H=-\frac{4}{f_{b}}\left(\frac{1}{32 \pi^{2} T^{2}}+Y_{2}^{(1 t)}+Y_{(1,1)}^{(2 t t)}\right)
$$

in such theories, where $Y_{2}^{(1 t)}$ and $Y_{(1,1)}^{(2 t t)}$ are given by expression (4.65), combined with eq. (B.4). A priori, $Y_{2}^{(1 t)}$ and $Y_{(1,1)}^{(2 t t)}$ depend on both background fields $A(u), f(u)$ and 
their boundary and horizon modes $\left\{A_{b}, f_{b}\right\}$ and $\left\{A_{H}, f_{H}\right\}$. Replacing $A(u)$ with its analytic solution, eq. (4.67),

$$
A(u)=\frac{1}{4} \log \left(\frac{2 f_{b} A_{b}^{2}}{u f^{\prime}(u)}\right)
$$

however, also cancels the explicit dependence on $A_{b}$ and $A_{H}$ in eq. (4.77) and $H$ becomes a function of $f_{b}$ and $f(u)$ only, though it depends on the latter through a complicated integral over a rational functional of $f(u)$ and its derivatives. Yet, close to the UV fixed point we can expand the integrand in the deviation $\delta f(u)$ from the conformal solution (4.31),

$$
\begin{aligned}
f(u) & =1-u^{2}+\delta f(u), \\
f(u \rightarrow 0) & \sim 1+\left(-1+\delta f_{b}\right) u^{2}, \quad f(u \rightarrow 1) \sim\left(2+\delta f_{H}\right)(1-u) .
\end{aligned}
$$

To linear order in $\delta f$, the result for $H$ is

$$
\begin{aligned}
H= & \frac{1}{2 \pi^{2} T^{2}}\left(-\frac{1}{4 f_{b}}+\frac{1}{4}+\right. \\
& \left.+\int_{0}^{1} \mathrm{~d} w\left[P(u) \delta f^{\prime \prime}(u)+Q(u) \delta f^{\prime}(u)+\left(Q^{\prime}(u)-P^{\prime \prime}(u)\right) \delta f(u)\right]\right),
\end{aligned}
$$

where we defined

$$
\begin{aligned}
& P(u) \equiv \frac{(1+u) \log (1+u)}{8 u^{2}}, \\
& Q(u) \equiv \frac{u\left(1+2 u-3 u^{2}\right)-(1+u)^{2}(2-3 u) \log (1+u)}{8 u^{3}\left(1-u^{2}\right)} .
\end{aligned}
$$

Integrating by parts and inserting the near-boundary and near-horizon behaviour of $\delta f(u)$, eq. (4.79b), one finds that $H$ indeed vanishes to first order in $\delta f$.

This proves that, even when taking into account the leading non-conformal corrections to second-order transport caused by an arbitrary scalar operator of dimension $\Delta=3$, the combination $H=2 \eta \tau_{\pi}-4 \lambda_{1}-\lambda_{2}$ remains zero in strongly coupled holographic fluids. 


\subsection{Numerical results for second-order transport}

This section contains our numerical results for second-order transport in non-conformal holographic fluids. In subsection 4.6.1 we present the leading non-conformal corrections to second-order hydro coefficients. They only depend on the mass term in the scalar potential and are therefore common to all holographic RG flows triggered by a scalar operator of dimension $\Delta=3$. In subsection 4.6 .2 we introduce two specific examples of holographic RG-flow families. We plot and discuss our numerical results for the transport coefficients along these flows in subsection 4.6.3.

\subsubsection{Leading non-conformal correction to second-order coeffi- cients}

All holographic RG flows described by (4.24) and (4.25) share the same UV fixed point, dual to the $A d S_{5}$ black-brane geometry (4.31) with vanishing scalar $\phi=0$. At high temperatures (compared to the scalar source $\Lambda$ ), $\phi$ remains close to zero and can be treated as a small perturbation of the conformal background. Its leading backreaction on the geometry occurs at quadratic order $\mathcal{O}\left(\phi^{2}\right)$ and can be computed analytically as we show in appendix B.3. The result only depends on the quadratic mass term in the bulk potential $V(\phi)$ and is therefore the same for all holographic RG flows triggered by a scalar operator of dimension $\Delta=3$.

Taking the result for the backreaction (eqs. (B.13), (B.14), (B.20), (B.22), (B.23)),

and plugging it into integrals (4.65) to compute the deviation of the $Y_{j}^{(a)}$ from their conformal values (4.66), we obtain the leading non-conformal corrections to the transport coefficients via eq. (4.74). We were not able to perform the required integrals over the backreaction analytically, but they are easily evaluated numerically.

Thanks to identities $\tilde{H}=2 \eta \tau_{\pi}-2\left(\kappa-\kappa^{*}\right)-\lambda_{2}=0$ and $H=2 \eta \tau_{\pi}-4 \lambda_{1}-\lambda_{2}=0$, which we proved to hold when taking into account leading non-conformal corrections in subsection (4.5.2), only three of the five transport coefficients (4.2) are independent. 
The results for $\kappa, \lambda_{2}$, and $\lambda_{1}+\lambda_{3} / 4$ are $^{12}$

$$
\begin{aligned}
\kappa & =2\left(\frac{s}{8 \pi^{2} T}\right)\left(1-4.5979 \cdot 10^{-3}(\Lambda / T)^{2}\right)+\mathcal{O}\left((\Lambda / T)^{4}\right), \\
\lambda_{2} & =-2 \log 2\left(\frac{s}{8 \pi^{2} T}\right)\left(1+4.9253 \cdot 10^{-3}(\Lambda / T)^{2}\right)+\mathcal{O}\left((\Lambda / T)^{4}\right), \\
\lambda_{1}+\lambda_{3} / 4 & =\left(\frac{s}{8 \pi^{2} T}\right)\left(1+2.5506 \cdot 10^{-3}(\Lambda / T)^{2}\right)+\mathcal{O}\left((\Lambda / T)^{4}\right),
\end{aligned}
$$

while the other two combinations of transport coefficients satisfy

$$
\eta \tau_{\pi}+\kappa^{*}=\kappa+\lambda_{2} / 2, \quad \lambda_{1}+\kappa^{*} / 2=\kappa / 2 .
$$

The numerical integration over the backreaction can be done to very high accuracy, but we chose to only display the first five digits in eq. (4.83). By checking the identity $H=0$ and by comparing results obtained when writing cancelling divergences in the integrands in different ways, we could estimate the absolute numerical error to be smaller than $10^{-14}$.

We conclude this subsection by emphasising again that the leading non-conformal corrections (4.83) and (4.84) are common to all holographic RG flows triggered by a scalar operator of dimension $\Delta=3$.

\subsubsection{Two simple families of holographic RG flows}

Potential $V_{(1)}$ : The first family $V_{(1)}$ of potentials that we are going to investigate has recently been introduced in ref. [171]. It derives from a family of quartic superpotentials $W$

$$
L W=-\frac{3}{2}-\frac{\phi^{2}}{8}+\frac{\phi^{4}}{16 \phi_{m}^{2}}
$$

resulting in

$$
\begin{aligned}
V_{(1)} & =8\left[\left(\frac{\partial W}{\partial \phi}\right)^{2}-\frac{2}{3} W^{2}\right] \\
& =\frac{1}{L^{2}}\left[-12-\frac{3}{2} \phi^{2}-\frac{1}{12} \phi^{4}+\frac{6+\phi_{m}^{2}}{12 \phi_{m}^{4}} \phi^{6}-\frac{1}{48 \phi_{m}^{4}} \phi^{8}\right] .
\end{aligned}
$$

\footnotetext{
${ }^{12}$ Note that $\lambda_{3}$, as opposed to $\kappa^{*}$, does not vanish for conformal fluids in general $[113,154]$. It does, however, vanish in conformal holographic theories at strictly infinite coupling [11].
} 
The potential $V_{(1)}$ has a maximum at $\phi=0$ and a minimum at the free parameter $\phi_{m}$. Close to $\phi=\phi_{m}$, the potential takes the form

$$
L^{2} V_{(1)}=-12 \frac{L^{2}}{L_{\mathrm{IR}}^{2}}+\frac{m_{\mathrm{IR}}^{2} L^{2}}{2}\left(\phi-\phi_{m}\right)^{2}+\mathcal{O}\left(\left(\phi-\phi_{m}\right)^{3}\right),
$$

yielding a second asymptotically $A d S_{5}$-region, dual to an IR fixed point, with a smaller AdS radius

$$
L_{\mathrm{IR}} \equiv\left(1+\frac{\phi_{m}^{2}}{24}\right)^{-1} L
$$

and a positive mass

$$
m_{\mathrm{IR}}^{2} L^{2}=12+\frac{\phi_{m}^{2}}{3}
$$

The potential $V_{(1)}$ thus represents a family of RG flows between a UV CFT, deformed by a relevant operator of dimension $\Delta=3$, and an IR CFT, whose number of degrees of freedom is smaller by a factor of $\left(L_{\mathrm{IR}} / L\right)^{3}$ compared to the UV [60,172] and which is deformed by an irrelevant operator of dimension $[2,3]$

$$
\begin{aligned}
\Delta_{\mathrm{IR}} & =2+2 \sqrt{1+\frac{m_{\mathrm{IR}}^{2} L_{\mathrm{IR}}^{2}}{4}} \\
& =4+\frac{48}{24+\phi_{m}^{2}} \in(4,6) .
\end{aligned}
$$

For smaller $\phi_{m}$, the number of degrees of freedom in the IR increases and the operator becomes more irrelevant in the IR. In this sense, the RG flow happens more quickly. In the opposite limit $\phi_{m} \rightarrow \infty$, the potential becomes quartic,

$$
V_{(1)} \stackrel{\phi_{m} \rightarrow \infty}{\longrightarrow} \frac{1}{L^{2}}\left[-12-\frac{3}{2} \phi^{2}-\frac{1}{12} \phi^{4}\right]
$$

the number of degrees of freedom in the IR goes to zero, and the IR operator becomes marginally irrelevant. In this sense, the RG flow happens infinitely slowly.

Potential $V_{(2)}$ : The second family $V_{(2)}$ of potentials that we are going to study is given by

$$
\begin{aligned}
V_{(2)} & =\frac{1}{L^{2}}\left[-12-\left(\frac{3}{2}-\frac{1}{\gamma^{2}}\right) \phi^{2}+\frac{2}{\gamma^{4}}(1-\cosh (\gamma \phi))\right] \\
& =\frac{1}{L^{2}}\left[-12-\frac{3}{2} \phi^{2}-\frac{1}{12} \phi^{4}-\frac{\gamma^{2}}{360} \phi^{6}+\mathcal{O}\left(\phi^{8}\right)\right] .
\end{aligned}
$$



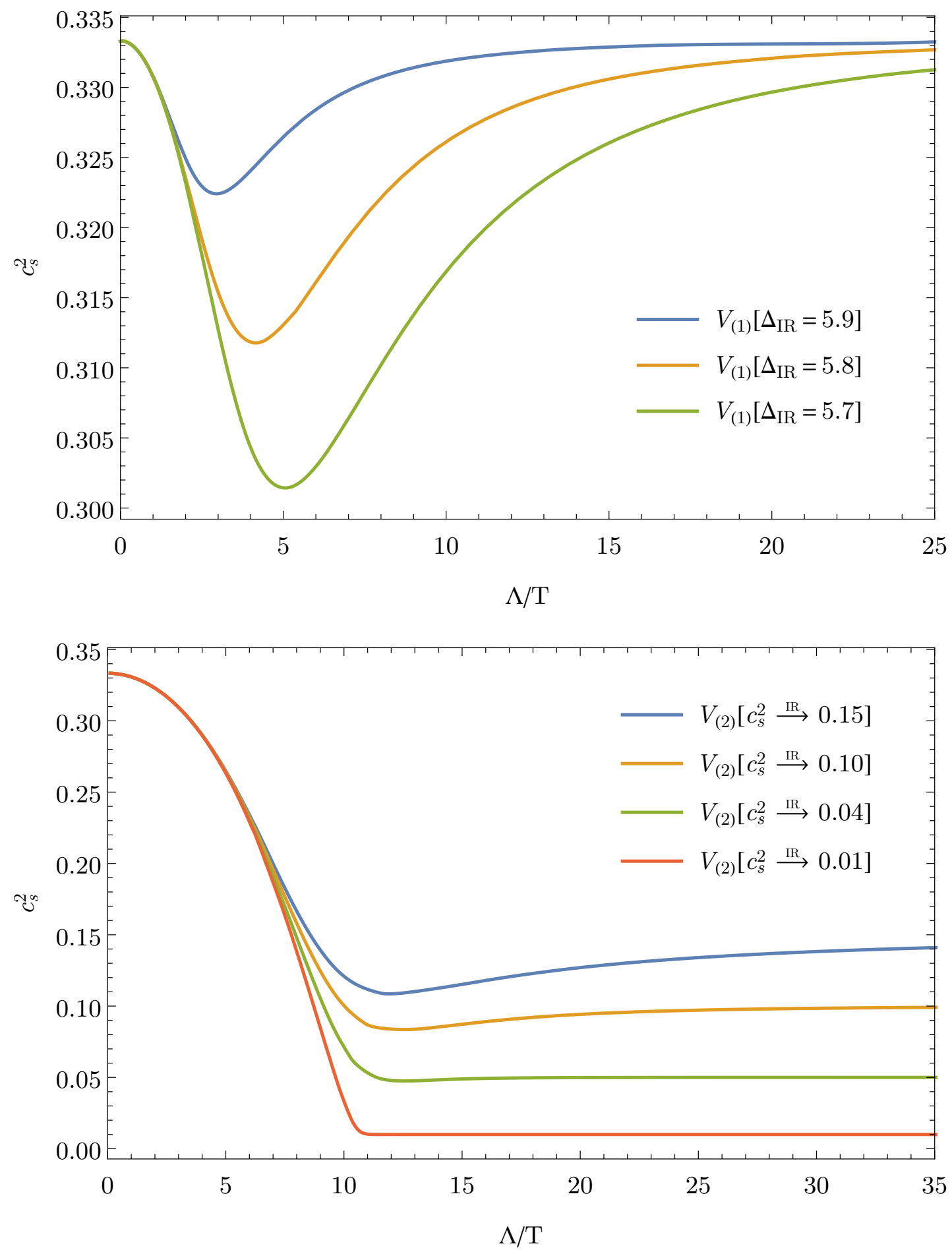

Figure 4.1: The speed of sound squared $c_{s}^{2}$ plotted versus operator source $\Lambda$ over temperature $T$. The upper plot shows our numerical results for potential $V_{(1)}$ for three operator dimensions $\Delta_{\mathrm{IR}}$ at the IR fixed point, eq. (4.90). The lower plot shows our numerical results for potential $V_{(2)}$ for four values of the parameter $\gamma$, which determines the value of $c_{s}^{2}$ in the deep IR via eq. (4.94), $c_{s}^{2} \rightarrow 1 / 3-\gamma^{2} / 2$. Each curve represents a holographic RG flow triggered by a different operator. 
The potential $V_{(2)}$ is monotonically decreasing for any value of the free parameter $\gamma$ and hence represents an RG flow from a UV CFT towards a non-conformal IR.

For large $\phi, V_{(2)}$ asymptotically approaches an exponential potential, $L^{2} V_{(2)} \rightarrow$ $-e^{\gamma \phi} / \gamma^{4}$, for which the finite-temperature solution is given by the analytically known Chamblin-Reall background $[142,173]$. In the deep IR, i.e. for large values $\phi_{H}$ of the scalar at the horizon, the near-horizon region of solutions to our model $V_{(2)}$ is therefore asymptotically described by the Chamblin-Reall background. In particular, temperature $T$ and entropy density $s$ take the following form in the limit of large $\phi_{H}[142]:$

$$
\begin{aligned}
\log (L T) & =\left(\frac{\gamma}{2}-\frac{1}{3 \gamma}\right) \phi_{H}+\left(\text { const in } \phi_{H}\right) \\
\log \left(4 G_{N} s\right) & =-\frac{\phi_{H}}{\gamma}+\left(\text { const in } \phi_{H}\right) .
\end{aligned}
$$

This implies that the speed of sound $c_{s}$ in the deep IR is

$$
c_{s}^{2}=\frac{\mathrm{d} \bar{p}}{\mathrm{~d} \bar{\epsilon}}=\frac{\mathrm{d} \log T}{\mathrm{~d} \log s} \stackrel{T \rightarrow 0}{\longrightarrow} \frac{1}{3}-\frac{\gamma^{2}}{2} .
$$

Importantly, black-brane solutions to $V_{(2)}$ can be stable for arbitrarily small temperatures only if $c_{s}^{2}>0$, i.e. if $|\gamma|<\sqrt{2 / 3} .{ }^{13}$ Note that for $\gamma \rightarrow 0, V_{(2)}$ approaches the same quartic potential as $V_{(1)}$ does in the limit $\phi_{m} \rightarrow \infty$, eq. (4.91):

$$
V_{(2)} \stackrel{\gamma \rightarrow 0}{\longrightarrow} \frac{1}{L^{2}}\left[-12-\frac{3}{2} \phi^{2}-\frac{1}{12} \phi^{4}\right] .
$$

Background solutions: For both families $V_{(1)}$ and $V_{(2)}$ we used the method devised in ref. [142] to construct numerical background solutions. The essential steps are summarised in appendix B.4. Figure 4.1 shows our numerical results for the speed of sound along the two $\mathrm{RG}$ flows for a few representative values of the respective parameters $\Delta_{\mathrm{IR}}$ and $\gamma$.

\footnotetext{
${ }^{13}$ Note that this implies that the $G P P Z$ flow [157] with superpotential $W=-\frac{3}{4}(1+\cosh (\phi / \sqrt{3}))$ does not admit stable black-brane solutions below a certain minimum temperature as its potential approaches $V \rightarrow-(3 / 8) \exp (2 \phi / \sqrt{3})$ for large $\phi$.
} 

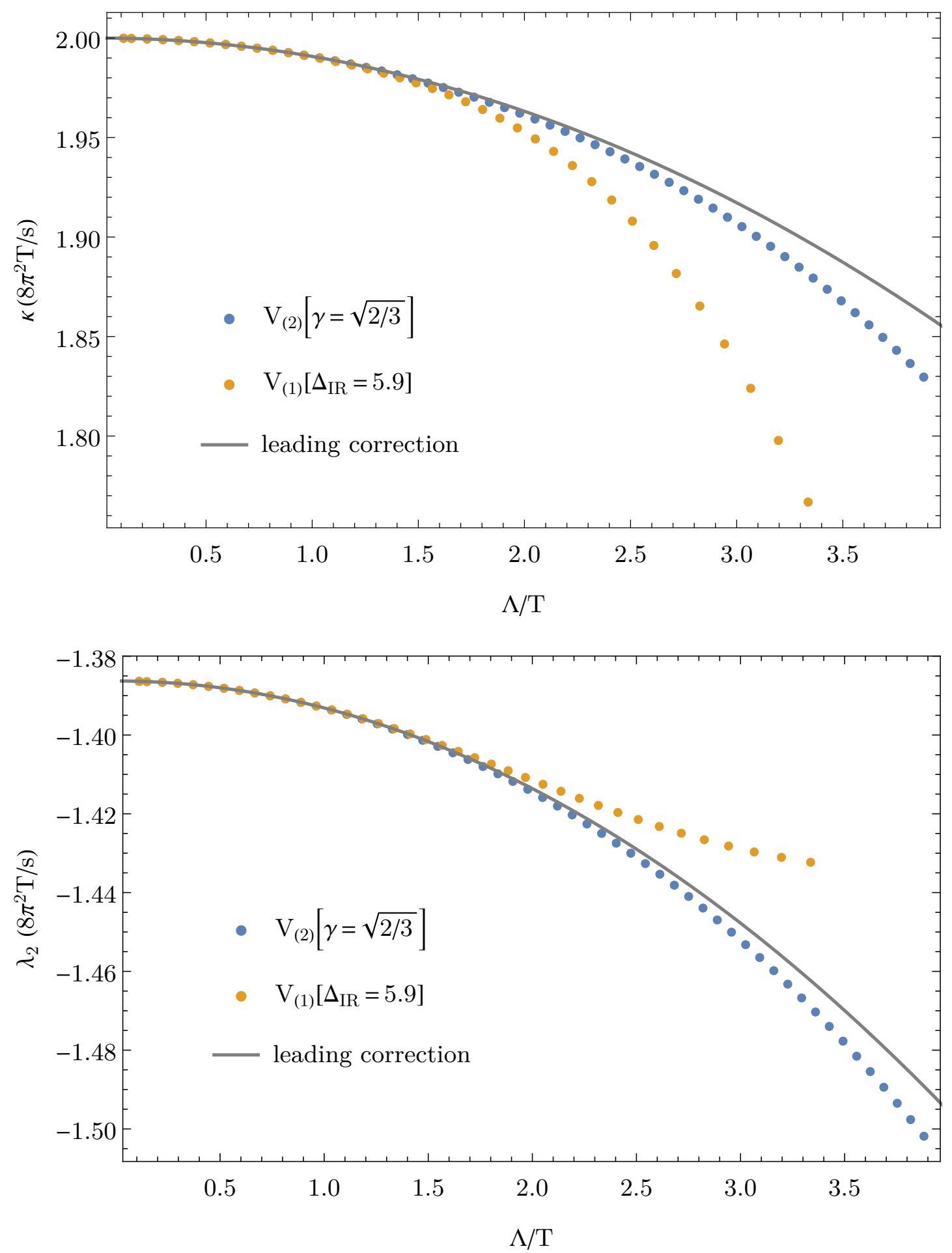

Figure 4.2: The second-order coefficients $\kappa$ and $\lambda_{2}$ in units of $s /\left(8 \pi^{2} T^{2}\right)$ plotted versus $\Lambda / T$. The plots show our numerical results for $V_{(1)}$ and $V_{(2)}$ with $\Delta_{\mathrm{IR}}=5.9$ and $\gamma=\sqrt{2 / 3}$ respectively. For smaller values of $\Delta_{\mathrm{IR}}$ and $\gamma$, the orange and blue curve move closer to each other until they coincide for $\Delta_{\mathrm{IR}} \rightarrow 4$ and $\gamma=0$, see eqs. (4.91) and (4.95). The solid line describes the leading non-conformal corrections (4.83) from subsection 4.6.1. 

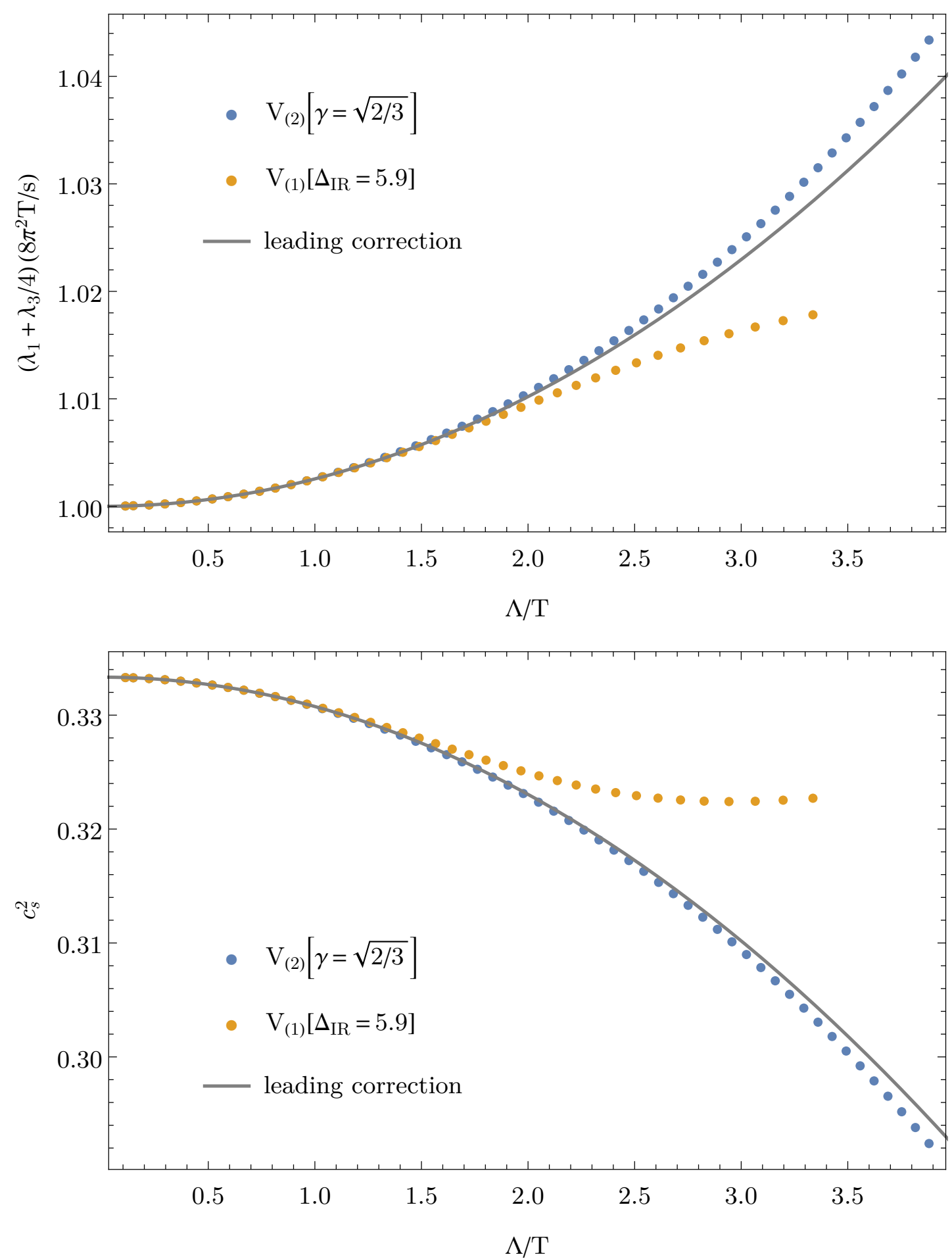

Figure 4.3: The second-order coefficient $\lambda_{1}+\lambda_{3} / 4$ in units of $s /\left(8 \pi^{2} T^{2}\right)$ and the speed of sound squared $c_{s}^{2}$ plotted versus $\Lambda / T$. The plots show our numerical results for $V_{(1)}$ and $V_{(2)}$ with $\Delta_{\mathrm{IR}}=5.9$ and $\gamma=\sqrt{2 / 3}$ respectively. For smaller values of $\Delta_{\mathrm{IR}}$ and $\gamma$, the orange and blue curve move closer to each other until they coincide for $\Delta_{\mathrm{IR}} \rightarrow 4$ and $\gamma=0$, see eqs. (4.91) and (4.95). The solid line describes the leading non-conformal corrections (4.83) from subsection 4.6.1. 


\subsubsection{Second-order coefficients along examples of RG flows}

This section contains our numerical results for the second-order coefficients (4.2) along the two families of holographic RG flows introduced in subsection 4.6.2. For each of the two families $V_{(1)}$ and $V_{(2)}$, we looked at around 20 parameter values covering the range $4.1 \leq \Delta_{\mathrm{IR}} \leq 5.9$ and $0 \leq \gamma \leq \sqrt{2 / 3}$ respectively. For each of these flows we then constructed numerical background solutions at about 40 different temperatures and computed the second-order coefficients from eq. (4.74).

Our main result is that the combination $H=2 \eta \tau_{\pi}-4 \lambda_{1}-\lambda_{2}$ vanishes in all cases considered, even when the individual transport coefficients deviate from their conformal values by factors of two and more. More precisely, the absolute values we obtained for $H$ all lie below our numerical accuracy of order $10^{-5}$. For details on the numerics see the end of appendix B.4. Our result suggests that the identity $H=0$ does not only hold for holographic fluids with conformal symmetry [14,136-139] or close to a fixed point (see ref. [140] and our subsection 4.5.2), but is in fact universally satisfied by all holographic fluids at infinite coupling, with or without conformal symmetry.

Combined with $\tilde{H}=2 \eta \tau_{\pi}-2\left(\kappa-\kappa^{*}\right)-\lambda_{2}=0$, the result $H=0$ implies that only three of the five coefficients (4.2) are independent. In figures 4.2 and 4.3 we plot the second-order coefficients $\kappa, \lambda_{2}, \lambda_{1}+\lambda_{3} / 4$ and the speed of sound squared $c_{s}^{2}$ versus $\Lambda / T$. The plots show our numerical results for $V_{(1)}$ and $V_{(2)}$, each with the largest parameter value considered, i.e. $\Delta_{\mathrm{IR}}=5.9$ and $\gamma=\sqrt{2 / 3}$. Our results for smaller parameter values all lie between these two extreme curves and vary smoothly with $\Delta_{\mathrm{IR}}$ and $\gamma$. The plots confirm that the behaviour of the transport coefficients close to the UV fixed point, i.e. for small $\Lambda / T$, is well described by the leading nonconformal correction discussed in subsection 4.6.1. If we compare figures 4.2 and 4.3 with figure 4.1, the difference in the considered range of $(\Lambda / T)$-values stands out. In particular, while figure 4.1 follows the speed of sound all the way from the UV to the IR region, figures 4.2 and 4.3 only contain results for relatively high $T$ and do not capture the IR properties of $V_{(1)}$ and $V_{(2)}$. This is due to the fact that we did not use the same radial coordinate to compute the transport coefficients that we had used to compute thermodynamic quantities such as $c_{s}^{2}$. The latter were obtained using the 
scalar $\phi$ itself as radial coordinate, as required by the method we employed to construct background solutions [142], see appendix B.4 for details. However, $\phi$ is not a suitable coordinate in the UV, where it becomes small everywhere. In particular, it does not lend itself to a perturbative treatment as in subsection 4.6.1 and it is ill-defined in the conformal limit $\phi \rightarrow 0$. For this reason, we switched to the $u$-coordinate when dealing with metric fluctuations around the background. While $u$ is well-defined in the UV, it becomes problematic in the IR because the region $\phi \in\left(0, \phi_{H}\right)$ is mapped onto the same interval $u \in(0,1)$ for all values of $\phi_{H}$. At low $T$, i.e. for large $\phi_{H}$, the modes in the $u$-coordinate become very large and render the numerics unstable. Nonetheless, we decided to work in the $u$-coordinate as it allowed us to obtain independent results from the perturbative treatment of $\phi$ and to compare every step of our calculations with the conformal case. The drawback is that reliable results for the transport coefficients could only be obtained for relatively small values of $\Lambda / T$. In particular, we cannot observe how the transport coefficients go back to their conformal values in the case of $V_{(1)}$ or begin to approach the values assumed in the Chamblin-Reall background in the case of $V_{(2)}$ (see appendix F of ref. [13]). We leave the numerical investigation of second-order coefficients in the deep IR for future research.

Let us take another look at figure 4.1. It indicates that for $V_{(2)}$ the influence of the IR becomes dominant only if $\Lambda / T \gtrsim 10$. We found that the same is true for $V_{(1)}$ with $\Delta_{\text {IR }} \lesssim 5.2$. In these cases it was therefore possible to obtain reliable numerical results for larger values of $\Lambda / T$ than it was in the case of $V_{(1)}$ with $\Delta_{\text {IR }}$ close to 6 . Figure 4.4 shows the deviations of $c_{s}^{2}, \kappa, \lambda_{2}$, and $\lambda_{1}+\lambda_{3} / 4$ from their conformal values for $V_{(2)}$ with $\gamma=\sqrt{2 / 3}$ and $\gamma=0$, plotted against $\Lambda / T$. Results for $V_{(2)}$ with $0<\gamma<\sqrt{2 / 3}$ lie between these two curves. Results for $V_{(1)}$ with $\Delta_{\mathrm{IR}} \lesssim 5.2$ closely follow the curve for $\left.V_{(2)}\right|_{\gamma=0}$, in agreement with $\left.V_{(1)} \stackrel{\Delta_{\mathrm{IR}} \rightarrow 4}{\longrightarrow} V_{(2)}\right|_{\gamma=0}$ from eqs. (4.91) and (4.95).

Finally, let us briefly mention the constraints from the local entropy current. By demanding that the entropy current's gradient expansion only contains terms that always lead to non-negative entropy production, refs. [93, 174] obtained five relations between second-order coefficients. ${ }^{14}$ We employed these relations in section 6.4 of ref. [13] to

\footnotetext{
${ }^{14}$ The same relations were found in ref. [175] by coupling the fluid to external sources.
} 


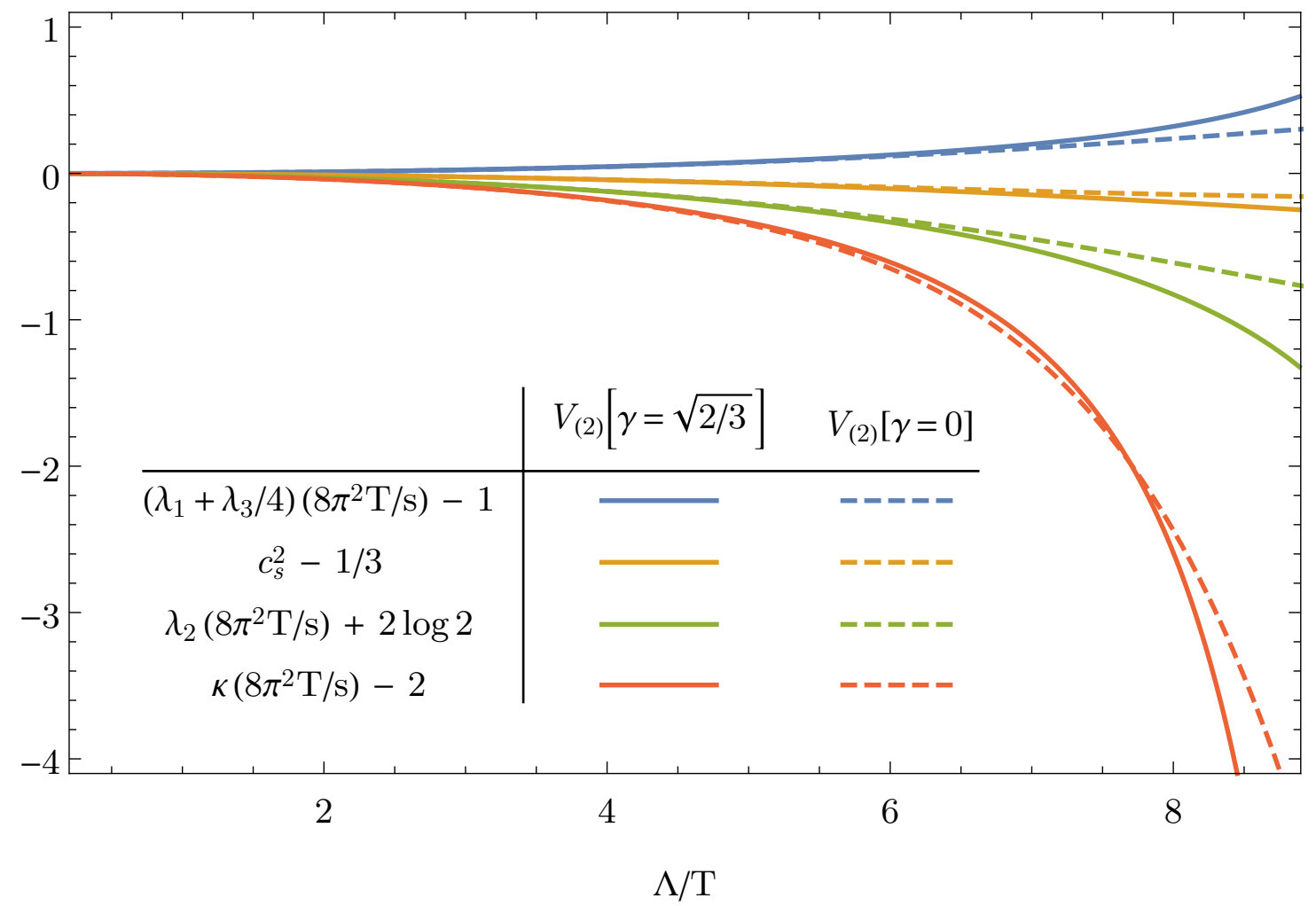

Figure 4.4: The deviation of the speed of sound squared $c_{s}^{2}$ and the second-order coefficients $\lambda_{1}+\lambda_{3} / 4, \lambda_{2}, \kappa$ in units of $s /\left(8 \pi^{2} T\right)$ from their conformal values (4.76), versus $\Lambda / T$. The solid line shows our numerical results for $V_{(2)}$ with $\gamma=\sqrt{2 / 3}$, the dashed line refers to $V_{2}$ with $\gamma=0$. Results for intermediate values of $\gamma$ interpolate smoothly between the two lines.

extend our numerical results to eight second-order coefficients, see figure 5 therein.

\subsection{Summary and outlook}

In this chapter we studied second-order hydrodynamic transport in strongly coupled non-conformal field theories. We derived new Kubo formulae for five second-order transport coefficients valid for uncharged non-conformal fluids in $(3+1)$ dimensions. We applied these Kubo formulae to holographic RG flows triggered by a relevant scalar operator of dimension $\Delta=3$ and found expressions for the five second-order transport coefficients at infinite coupling in terms of the holographically dual gravitational background solution. We showed that the relation

$$
\tilde{H}=2 \eta \tau_{\pi}-2\left(\kappa-\kappa^{*}\right)-\lambda_{2}=0
$$


is satisfied along all such holographic RG flows. We proved that the Haack-Yarom identity $[14,135]$

$$
H=2 \eta \tau_{\pi}-4 \lambda_{1}-\lambda_{2}=0
$$

which is known to hold for conformal holographic fluids at infinite coupling, is also satisfied when leading-order non-conformal corrections are included within the class of considered RG flows. For the two specific classes of RG flows we studied numerically, we found that, all along each flow, the Haack-Yarom identity was also satisfied beyond perturbative non-conformal corrections. This provides further evidence that the identity may be universally satisfied by strongly coupled fluids [136-140,143].

We derived the new set of Kubo formulae for five second-order transport coefficients of non-conformal fluids in section 4.2, eq. (4.21), and showed how these Kubo formulae can be applied to strongly coupled fluids with a gravity dual. In section 4.3, we introduced a specific class of strongly coupled non-conformal field theories that describe an RG flow induced in the UV by a scalar operator of dimension $\Delta=3$. We derived bulk equations of motion both for asymptotically AdS backgrounds of the dual Einstein-scalar models and for the metric fluctuations that are relevant for the Kubo formulae. In section 4.4 we solved the fluctuation equations in a hydrodynamic derivative expansion and derived explicit integral expressions for the sub-leading modes in terms of background data, eq. (4.65). In section 4.5 we presented our analytical results for second-order transport coefficients. We determined the expressions (4.74) for five transport coefficients in terms of the dual background data and proved that a certain linear combination of second-order transport coefficients, $\tilde{H}=2 \eta \tau_{\pi}-2\left(\kappa-\kappa^{*}\right)-\lambda_{2}$, vanishes identically along any RG flow in the class considered. We showed that the Haack-Yarom identity $H=0$, eq. (4.1), is obeyed to leading order in the deviation from conformality for arbitrary holographic RG flows triggered by a relevant scalar operator of dimension $\Delta=3$. In section 4.6 we presented our numerical results for second-order transport coefficients. Firstly, we computed the leading non-conformal corrections to the second-order coefficients. Secondly, we introduced two specific families of $\Delta=3$ operators and numerically found the Haack-Yarom identity to be obeyed along both families of corresponding RG flows. Thirdly, we plotted the independent combinations 
of second-order coefficients along both classes of $\mathrm{RG}$ flows and found them to agree with the perturbative results in the appropriate high-temperature regime.

Our work points to a number of open questions. For technical reasons, we restricted our bulk computations to transverse-vector and transverse-tensor perturbations of the metric. If scalar sound perturbations were included as well and an appropriate set of Kubo formulae were derived, it would be possible to compute all fifteen second-order non-conformal transport coefficients. The major technical obstacle is that these scalar sound perturbations necessarily source fluctuations of the scalar field in the bulk. If, despite this, one managed to compute all fifteen second-order coefficients, one would be able to check the five relations which were derived from the positivity of the local entropy production [93, 174, 175].

In presenting our numerical results in subsection 4.6 .3 we pointed out that our choice of the radial coordinate $u$ made it impossible to numerically access the deep IR of the considered RG flows, despite several other advantages. As we explain in appendix B.4, the IR region would become accessible if one used the scalar $\phi$ as radial coordinate instead. We have precise expectations as to what such a numerical study would reveal. For the first family of potentials the transport coefficients have to return to their conformal values. For the second family of potentials they are expected to approach the values they assume in the Chamblin-Reall background, which are listed in appendix $\mathrm{F}$ of ref. [13].

Whether our proof in subsection 4.5.2 that $H$ vanishes when taking into account leading non-conformal corrections caused by an operator of dimension $\Delta=3$ can be generalised to relevant operators of arbitrary dimension $2<\Delta<4$ is another open question. Since the computation of $H$ does not involve fluctuations of the bulk scalar, a change in the dual operator dimension would require a different set of holographic counterterms but would not affect the relevant bulk metric fluctuations.

What our results entail for the entropy current is another direction for future research. Fluids with simple gravity duals at strong coupling have been conjectured to obey a principle of minimal dissipation $[136,176]$. The observation that the lower bound on the shear viscosity over entropy density ratio is universally satisfied by a large class 
of holographic theories $[7,67,94,121-126]$ is a first hint in this direction, since this ratio appears as coefficient of the leading contribution to the entropy production. At second order in gradients, the entropy current of a conformal fluid contains two terms [93]: the coefficient of the first one vanishes if $2 \lambda_{1}=\kappa$, which is indeed true for conformal holographic fluids at infinite coupling [10], while the coefficient of the second term remains unknown. The relations $\tilde{H}=0$ and $H=0$ found in this work are equivalent to $H=0$ and $2 \lambda_{1}=\kappa-\kappa^{*}$. It was shown within an effective action approach to adiabatic hydrodynamics that these two relations must indeed hold for perfect fluids that do not produce entropy [177]. In fact, the Haack-Yarom identity $H=0$ seems to require either adiabaticity or infinite coupling. Even for conformal fluids, the identity is violated in examples of weakly coupled systems in the kinetic regime [178] and when finite coupling corrections are included in the hypothetical dual of Gauss-Bonnet gravity $[136,138,139] .{ }^{15}$ It would be interesting to explore whether the relations $H=0$ and $2 \lambda_{1}=\kappa-\kappa^{*}$ lead to cancellations in the divergence of the non-conformal entropy current, similar to the conformal case. This would provide further evidence in favour of the principle of minimal dissipation.

\footnotetext{
${ }^{15}$ Consistently, $H=0$ does not follow from the generalised Onsager relations, which were derived from an effective action for hydrodynamics in refs. $[179,180]$ and which should apply to any uncharged conformal fluid.
} 


\section{Chapter 5}

\section{A Holographic Kondo Model}

\section{$5.1 \quad$ Introduction and summary}

We mentioned in section 3.3 that the ill-understood strange metal phase, which is most likely controlled by a strongly coupled critical theory, provides one of the main incentives to apply the gauge/gravity duality to condensed matter systems. Strange metal phases, whose characteristic feature is a resistivity $\rho \propto T$ [181], are observed in cuprate superconductors and in many heavy fermions. The latter are rare-earth or actinide based alloys, many of which exhibit a continuous quantum phase transition from an anti-ferromagnetic (AFM) phase to a Landau Fermi Liquid (LFL) phase ${ }^{1}$ with quasi-particles hundreds of times heavier than those of normal metals, hence the name 'heavy fermions' [181-186]. The quantum critical degrees of freedom appear to be strongly interacting [187] and, when heated up, give rise to a strange metal.

Doniach [188] proposed the Kondo lattice as a theoretical description of heavy fermions. The rare-earth or actinide atoms' $f$-orbital electrons form a lattice of localised spin impurities $S_{i}$, while the other atoms provide conduction electrons that form a LFL. The Kondo lattice Hamiltonian thus includes a LFL kinetic term plus two types of interactions: Kondo interactions $\lambda_{\mathrm{K}} S_{i} \cdot \mathcal{J}$ between the impurities $S_{i}$ and the spin current $\mathcal{J}$ of the LFL (see eq. (5.1)), and Heisenberg interactions $\lambda_{\text {RKKY }} S_{i} \cdot S_{j}$ between neighbouring impurities, called Ruderman-Kittel-Kasuya-Yosida (RKKY) in-

\footnotetext{
${ }^{1}$ A LFL describes weakly interacting fermionic quasi-particles ('dressed electrons') whose excitations are in one-to-one correspondence with and have the same quantum numbers as their free counterparts ('bare electrons'), but whose mass and other couplings assume different, renormalised values [105].
} 
teractions (see eq. (5.10)). A solution to the Kondo lattice Hamiltonian is only known in simplifying limits. The simplest limits just ignore one or the other type of interaction. If the Kondo interactions can be neglected, then AFM RKKY couplings $\left(\lambda_{\text {RKKY }}>0\right)$ trivially lead to an AFM metal. Conversely, suppose the impurities are sufficiently dilute so that the RKKY interactions can be neglected. In that limit, the relevant dynamics is captured by the Kondo coupling of the LFL to a single spin. The corresponding single-impurity Kondo Hamiltonian [189] (see subsection 5.2.1) has been realised experimentally in metals doped with a dilute concentration of magnetic impurities [189-191] and in quantum dots [192-194]. Most famously, in doped metals the single-impurity Kondo model successfully describes the logarithmic rise of the resistivity $\rho$ with decreasing temperature $T$ [189]. The single-impurity Kondo model has been studied using a variety of complementary techniques, such as numerical RG [195-197], integrability [198-205], large- $N$ limits [181, 182, 206-209], and CFT [209-215]. Many of these are reviewed in refs. [216,217]. The solution is most succinctly described as an RG flow. An AFM Kondo coupling $\left(\lambda_{\mathrm{K}}>0\right)$ flows to larger values in the IR and eventually diverges at a dynamically generated scale, the Kondo temperature $T_{\mathrm{K}}$. At low $T$, the impurity is screened by a Kondo cloud [218] of LFL fermions, the fermionic spectral function exhibits a Kondo resonance at the Fermi level, and the fermions acquire an $s$-wave phase shift of $\pi / 2$ [216,217]. The term 'Kondo effect' is used to refer to all of these phenomena. ${ }^{2}$

Clearly, simplifying limits of the Kondo lattice Hamiltonian that ignore either the Kondo or the RKKY interaction cannot describe the quantum critical point, which arises from a competition between the two. As a first step towards the Kondo lattice, the two-impurity Kondo model [206,219-236] (see subsection 5.2.2) provides the simplest example that features the competition between Kondo and RKKY interactions. It has not been solved in full generality, but substantial progress has been made using a combination of methods, including large- $N$ [206, 224, 225] and CFT [227-232]. For example, a quantum phase transition has been found to occur for an $S U(2)$ spin symmetry [221,222]. In that case, a large ferromagnetic (FM) RKKY coupling forces

\footnotetext{
${ }^{2}$ Intuitively, a conduction electron locks with the impurity into a spin singlet, which makes the impurity location impenetrable for other conduction electrons due to Pauli exclusion.
} 
the spins to lock into a spin-1 triplet, which at low $T$ is screened in a multi-stage Kondo effect. For large AFM RKKY coupling, the spins lock into a singlet and decouple from the LFL. The LFL fermions thus acquire a $\pi / 2$ phase shift in the FM phase where the Kondo effect occurs, but no phase shift in the AFM phase. At $T=0$, particle-hole symmetry only allows phase shifts of $\pi / 2$ or 0 so that the phase shift must jump discontinuously at a quantum phase transition [225], which turns out to be second-order [221,222, 227, 235, 236].

Yet many open questions remain about the single- and two-impurity Kondo models, in particular regarding the fate of the Kondo effect in the case of strongly correlated electrons, e.g. in a Luttinger liquid [237-241] or in the Hubbard model [242,243]. These questions are of particular relevance in view of the strongly coupled nature of the strange metal, but cannot be reliably answered with existing techniques. We thus turn to an alternative approach using the gauge/gravity duality or holography [13]. This approach replaces the global $S U(2)$ spin symmetry by gauged $S U(N)$ and effectively replaces the LFL of the original Kondo model by a strongly coupled CFT with a holographic gravity dual, which can serve as a useful toy model for strongly correlated electrons. Various holographic single-impurity Kondo models exist, see e.g. refs. [18,244-257]. In all cases, the $S U(2)$ spin symmetry is replaced by a gauged $S U(N)$ symmetry, followed by the limits of large $N$ and infinite gauge coupling. Magnetic impurities are then described by an $S U(N)$ Wilson line [57, 77, 258-261]. ${ }^{3}$ Various attempts to build holographic lattices of impurities can be found in refs. [244-247, 250,263]. However, these include neither the Kondo nor the RKKY coupling, so it is unclear whether they really describe the strange metal state that arises from heavy fermion quantum criticality. Among holographic single-impurity Kondo models, only the model of ref. [18] includes a Kondo coupling at all. It also describes many essential single-impurity Kondo phenomena such as the appearance of $T_{\mathrm{K}}$ and a phase shift.

In this chapter, based on refs. [15-17], we further the analysis of the latter model in two ways. Firstly, we extend it to a holographic two-impurity Kondo model with RKKY inter-impurity coupling, and study the corresponding phase diagram [15]. Secondly,

\footnotetext{
${ }^{3} \mathrm{~A}$ different approach, using a delta-function source to describe a point-like impurity, is followed in ref. [262].
} 
we consider time-dependent fluctuations in the single-impurity case and compute the corresponding spectral functions [16,17]. The holographic model from ref. [18] is based on the CFT and the large- $N$ approaches to the Kondo model. In the CFT description, the Kondo model consists of chiral fermions $\psi$ in $(1+1)$ dimensions (representing radial electron $s$-waves), coupled to an impurity at the origin. The impurity is in a totally antisymmetric representation of the $S U(N)$ spin symmetry and can be represented in terms of Abrikosov pseudo-fermions $\chi$, which transform under an auxiliary $U(1)$ symmetry. Two impurities introduce two flavours of $\chi$ and enhance the auxiliary symmetry to $U(2)$. At large $N$, the Kondo effect appears as condensation of the operator $\mathcal{O}=\psi^{\dagger} \chi$ in a mean-field phase transition below a critical temperature. Our holographic model gauges the $S U(N)$ spin symmetry and employs the limit of large gauge coupling, thereby replacing the free UV fixed point of the original Kondo model by a strongly coupled CFT with a gravity dual in $A d S_{3}$. The electric current of the chiral $\psi$ is dual to a Chern-Simons gauge field in $A d S_{3}$. The auxiliary current of the $\chi$ and the impurity operator $\mathcal{O}$ are dual to a Yang-Mills (YM) gauge field $a$ and a scalar field $\Phi$ on an $A d S_{2}$ defect at the impurity's location.

We have six main results. One, we find that the RKKY interaction between totally anti-symmetric $S U(N)$ impurity spins can be written as a double-trace deformation at large $N$. This result holds independently of holography. Two, we identify the Kondo and RKKY couplings in our holographic model as boundary conditions on the fields $\Phi$ and $a$ in the dual AdS gravity bulk. This is the first identification of an inter-impurity coupling in holography. Three, we demonstrate that, at large $N$ and for totally antisymmetric $S U(N)$ impurity spins, FM spin-spin correlations are suppressed relative to AFM spin-spin correlations. This result is only based on $S U(N)$ representation theory and is valid independently of a holographic description. Four, a numerical investigation of the phase diagram in our model suggests that a quantum phase transition occurs at a non-trivial AFM value of the RKKY coupling, from a trivial phase with uncorrelated impurities and no Kondo screening to a non-trivial phase with AFM spin-spin correlations and simultaneous Kondo screening. Five, studying correlation functions in the single-impurity case, we observe a Fano resonance in $\mathcal{O}$ 's spectral function, which 
we identify with the Kondo resonance in the Kondo-screened low-temperature phase. Fano resonances occur because a symmetric resonance, in our case produced by the marginally relevant Kondo coupling, couples to a continuous spectrum, in our case provided by the impurity's $(0+1)$-dimensional scale invariance in the UV. Six, we propose that, following the same mechanism, Fano resonances should be a generic feature of RG flows between effectively $(0+1)$-dimensional fixed points.

The remainder of this chapter is organised as follows: In section 5.2 we review relevant aspects of the original single-and two-impurity Kondo model and show that the RKKY interaction takes the form of a double-trace deformation at large $N$. Section 5.3 describes our holographic gravity model. In section 5.4 we identify the Kondo and RKKY couplings as boundary conditions on fields in AdS. Section 5.5 contains our results for the two-impurity phase diagram. Section 5.6 discusses correlation functions in the single-impurity case. We conclude in section 5.7 with a summary of our results and suggestions for future research.

\subsection{The single- and two-impurity Kondo models}

In this section we review the details of the single- and two-impurity Kondo models that we will need for our holographic model.

\subsubsection{The single-impurity Kondo model}

The single-impurity Kondo model describes the interaction of a LFL with a single localised quantum impurity spin [216,217]. It has been realised experimentally in quantum dots [192-194] and in metals doped with a dilute concentration of magnetic impurities [189-191]. In many of these cases, multiple conduction bands or 'channels' couple to the same impurity, and in many cases the impurity has a spin degeneracy greater than two $[181,182,206,207,216,217]$. To describe these cases, the generalised Kondo model contains $k$ fermion channels, each in the fundamental representation of an $S U(N)$ spin symmetry, and an impurity $S^{A}$ in a general representation $\rho_{\mathrm{UV}}$ of $S U(N)$ with dimension $\operatorname{dim}\left(\rho_{\mathrm{UV}}\right)$. The symmetry group that leaves the Hamiltonian density invariant is then $S U(N) \times S U(k) \times U(1)$, with channel symmetry $S U(k)$ and 
electromagnetic symmetry $U(1)$. The single-impurity Kondo problem has been solved for general $N, k$ and $\rho_{\mathrm{UV}}$ using a number of complementary techniques, including numerical RG [195-197], integrability [198-205], large- $N$ techniques [181, 182,206-209], and CFT techniques [210-215]. Many of these are reviewed in refs. [216,217].

The single-impurity Kondo model is spherically symmetric about the impurity so that only $s$-waves couple to the impurity. The CFT approach [210-215] begins by discarding all higher partial waves (in real space), followed by linearising the dispersion relation about the Fermi momentum. The result is a $(1+1)$-dimensional relativistic model on the positive real axis, representing the radial distance to the impurity, with left- and right-moving fermions (in-coming and out-going $s$-waves) interacting with the impurity at the origin. The Fermi velocity plays the role of the speed of light, which we will henceforth set to one. After extending the positive real axis to negative values, reflecting the right-movers about the origin and re-labelling them as left-movers, we obtain the simplest description of the single-impurity Kondo model: left-movers alone, moving on the entire real line, interacting with the impurity at the origin. The resulting $(1+1)$-dimensional Hamiltonian density is (suppressing $S U(k)$ channel indices)

$$
H_{\mathrm{K}}=\frac{1}{2 \pi} \psi_{\alpha}^{\dagger} i \partial_{x} \psi_{\alpha}+\lambda_{\mathrm{K}} \delta(x) S^{A} \mathcal{J}^{A}, \quad \mathcal{J}^{A}=\psi_{\alpha}^{\dagger} T_{\alpha \beta}^{A} \psi_{\beta},
$$

where $\psi_{\alpha}^{\dagger}$ creates a left-moving fermion with spin $\alpha, \lambda_{\mathrm{K}}$ is the classically marginal Kondo coupling, and $T_{\alpha \beta}^{A}$ are the generators of $S U(N)\left(A=1, \ldots, N^{2}-1\right)$, in the fundamental representation. The free left-moving fermions form a chiral CFT, invariant under a single Virasoro algebra.

The beta function for the Kondo coupling is negative to one-loop order in perturbation theory $[216,217]$. Consequently, a FM Kondo coupling, $\lambda_{\mathrm{K}}<0$, is marginally irrelevant in the IR. On the other hand, an AFM Kondo coupling, $\lambda_{\mathrm{K}}>0$, is asymptotically free in the UV, but in the IR appears to diverge at a dynamically generated scale, the Kondo temperature, $T_{\mathrm{K}}$. In the high-temperature regime, $T \gg T_{\mathrm{K}}$, perturbation theory in $\lambda_{\mathrm{K}}$ is thus reliable for calculating observables, including (at one loop) the characteristic $-\ln \left(T / T_{\mathrm{K}}\right)$ contribution to the resistivity [189]. At low temperatures, the renormalisation of AFM $\lambda_{\mathrm{K}}$ to large values is the main obstacle to solving the Kondo problem, but we can reach a basic understanding of its solution as follows. Let 
us assume that, starting from the LFL in the UV, the RG flow takes us all the way to $\lambda_{\mathrm{K}} \rightarrow+\infty$ in the IR. In that case, the ground state must minimise the Kondo interaction $S^{A} \mathcal{J}^{A}$. Concretely, among all the eigenstates of $S^{A}+\mathcal{J}^{A}$ that can be formed by the impurity $\rho_{\mathrm{UV}}$ and the LFL fermions, subject to Pauli exclusion and $S U(k)$ channel symmetry, the ground state will have the minimal eigenvalue of $S^{A} \mathcal{J}^{A}[208,209]$. Let $\rho_{\mathrm{IR}}$ denote the corresponding $S U(N)$ representation of any impurity remaining in the IR, with dimension $\operatorname{dim}\left(\rho_{\mathrm{IR}}\right)$. The $\lambda_{\mathrm{K}} \rightarrow \infty$ fixed point must fall into one of the following three classes, depending on how $\operatorname{dim}\left(\rho_{\mathrm{IR}}\right)$ compares to $\operatorname{dim}\left(\rho_{\mathrm{UV}}\right)$ :

1. Critical Screening: If $\rho_{\mathrm{IR}}$ is a singlet of $S U(N), \operatorname{dim}\left(\rho_{\mathrm{IR}}\right)=0$, then the impurity has been screened completely. This occurs for instance in the original singlechannel $S U(2)$ Kondo model: the Kondo cloud has net spin 1/2, which locks with the impurity spin into the anti-symmetric singlet of $S U(2)$.

2. Underscreening: If $0<\operatorname{dim}\left(\rho_{\mathrm{IR}}\right) \leq \operatorname{dim}\left(\rho_{\mathrm{UV}}\right)$, then the impurity is either partially screened or unscreened. The net impurity spin that remains in the IR interacts with the LFL via a marginally irrelevant FM Kondo coupling [208, 264].

3. Overscreening: If $\operatorname{dim}\left(\rho_{\mathrm{IR}}\right)>\operatorname{dim}\left(\rho_{\mathrm{UV}}\right)$, then the naïve fixed point $\lambda_{\mathrm{K}} \rightarrow \infty$ cannot be the actual IR fixed point, since that would lead to a greater number of impurity degrees of freedom in the IR than in the UV, which is impossible for a physical RG flow [209,213]. In fact, the overscreened impurity interacts with neighbouring LFL fermions via a marginally relevant AFM Kondo coupling, rendering the naïve IR fixed point unstable. The true IR fixed point is at a non-trivial value of $\lambda_{\mathrm{K}}[264,265]$ and gives rise to non-Fermi liquid behaviour.

With critical or underscreening, the excitations about the ground state arrange themselves again into a LFL. However, the LFL fermions in the IR are subject to special boundary conditions: their wave function must vanish at the location of the impurity. Intuitively, the reason is that, due to Pauli exclusion, a LFL fermion can penetrate that location only by destroying the screened impurity in representation $\rho_{\mathrm{IR}}$, whose binding energy is $\propto \lambda_{\mathrm{K}} \rightarrow \infty$ [215]. The vanishing of the wave function is equivalent to an $s$-wave $\pi / 2$ phase shift in the IR relative to the UV. 
Large- $N$ Techniques: Our holographic model will employ a large- $N$ limit $[181,182$, 206-209], in which the Kondo effect appears as symmetry breaking at the impurity's location $[202,206,266,267]$. That description begins by representing the impurity spin $S^{A}$ in terms of Abrikosov pseudo-fermions in the fundamental representation of $S U(N)$ :

$$
S^{A}=\chi_{\alpha}^{\dagger} T_{\alpha \beta}^{A} \chi_{\beta}
$$

Here, $\chi_{\alpha}^{\dagger}$ and $\chi_{\alpha}$ are creation and annihilation operators for an Abrikosov pseudofermion. The $\chi_{\alpha}$ obey fermionic anti-commutation relations, which ensures that $S^{A}$ indeed obeys the $S U(N)$ algebra. The Hilbert space on which $S^{A}$ acts is built by acting on the vacuum with the $\chi_{\alpha}^{\dagger}$. Because the $\chi_{\alpha}^{\dagger}$ anti-commute, the states in the Hilbert space form totally anti-symmetric tensor products of the fundamental representation of $S U(N)$, with the rank of a tensor given by the total number $q$ of Abrikosov pseudofermions in a particular state (so $q$ ranges from zero to $N$ ). To obtain an irreducible representation, we must fix the rank $q$ of the anti-symmetric tensor by imposing a constraint on the states in the Hilbert space:

$$
\chi_{\alpha}^{\dagger} \chi_{\alpha}=q
$$

Note that we would obtain totally symmetric representations of $S U(N)$ if we represented $S^{A}$ via Schwinger bosons rather than Abrikosov pseudo-fermions [208, 268,269].

Using the completeness relation satisfied by the fundamental-representation $S U(N)$ generators,

$$
T_{\alpha \beta}^{A} T_{\gamma \delta}^{A}=\frac{1}{2}\left(\delta_{\alpha \delta} \delta_{\beta \gamma}-\frac{1}{N} \delta_{\alpha \beta} \delta_{\gamma \delta}\right)
$$

and $\chi_{\alpha}$ 's anti-commutation relations, we can re-write the Kondo interaction in eq. (5.1) as, after dropping an unimportant constant $\propto q$,

$$
\lambda_{\mathrm{K}} S^{A} \psi_{\gamma}^{\dagger} T_{\gamma \delta}^{A} \psi_{\delta}=\lambda_{\mathrm{K}}\left(\chi_{\alpha}^{\dagger} T_{\alpha \beta}^{A} \chi_{\beta}\right)\left(\psi_{\gamma}^{\dagger} T_{\gamma \delta}^{A} \psi_{\delta}\right)=-\frac{1}{2} \lambda_{\mathrm{K}}\left(\mathcal{O}^{\dagger} \mathcal{O}+\frac{q}{N}\left(\psi_{\alpha}^{\dagger} \psi_{\alpha}\right)\right)
$$

where we have defined the scalar operator $\mathcal{O} \equiv \psi_{\alpha}^{\dagger} \chi_{\alpha}$, which is a function of time $t$ only, because $\chi_{\alpha}$ cannot propagate away from the impurity's location $x=0$. Clearly, $\mathcal{O}$ is a singlet of the spin $S U(N)$ symmetry, has the same channel $S U(k)$ and electromagnetic $U(1)$ representation as $\psi_{\alpha}^{\dagger}$, and has the same auxiliary $U(1)$ charge as $\chi_{\alpha}$. Classically 
$\psi_{\alpha}$ has dimension $1 / 2$ and $\chi_{\alpha}$ has dimension zero, so $\mathcal{O}$ has dimension $1 / 2$. The Kondo interaction eq. (5.5) is then classically marginal, i.e. $\lambda_{\mathrm{K}}$ is classically dimensionless.

So far our discussion has been valid for any value of $N$, but let us now consider the large- $N$ limit: we take $N \rightarrow \infty$, keeping both $N \lambda_{\mathrm{K}}$ and $q / N$ fixed and of order one. In that limit, the $(q / N) \psi_{\alpha}^{\dagger} \psi_{\alpha}$ term eq. (5.5) is sub-leading and we find that the Kondo interaction is a classically-marginal 'double-trace' interaction, of the form $-\lambda_{\mathrm{K}} \mathcal{O}^{\dagger} \mathcal{O}$. We put 'double-trace' in quotation marks because $\mathcal{O}$ is not the trace of a matrix in the adjoint of $S U(N)$, but a contraction of a field in the anti-fundamental representation of $S U(N), \psi_{\alpha}^{\dagger}$, with a field in the fundamental representation, $\chi_{\alpha}$. In what follows we will drop the quotation marks. The double-trace form of the Kondo interaction will be extremely useful for our holographic model: a double-trace interaction will be realised holographically by a simple linear boundary condition on the complex scalar field dual to $\mathcal{O}$, as we will discuss in section 5.4.

The solution of the large- $N$ saddle point equations reveals a second-order meanfield phase transition at a critical temperature, on the order of but distinct from $T_{\mathrm{K}}$, below which $\mathcal{O}$ acquires a non-zero expectation value $\langle\mathcal{O}\rangle \neq 0[202,206,266,267]$. Intuitively, the condensation of $\mathcal{O}$ represents the formation of a Kondo cloud around $x=$ 0 , screening the impurity spin. Corrections in $1 / N$ will change the phase transition to a smooth cross-over [202], as observed in experimental realisations of the single-impurity Kondo effect [189-194]. In the large- $N$ limit, all equilibrium physics above the critical temperature, where $\langle\mathcal{O}\rangle=0$, reduces to that of the UV chiral CFT. Nevertheless, the large- $N$ limit captures much of the essential single-impurity Kondo physics at low $T$, including low- $T$ scaling exponents and the phase shift [181,182, 206-209].

To summarise: at low $T$ and large $N$, the single-impurity Kondo effect can be described as a $(1+1)$-dimensional chiral CFT, deformed by a marginally-relevant, double-trace coupling to an impurity spin.

\subsubsection{The two-impurity Kondo model}

The two-impurity Kondo model [206,219-236] is the simplest model that features the competition between the Kondo and RKKY interactions and is a natural first step 
towards building a Kondo lattice. It consists of two localised impurity spins $S_{\mathrm{I}}^{A}$ and $S_{\mathrm{II}}^{A}$, both in the same representation $\rho_{\mathrm{UV}}$ of $S U(N)$, separated by a distance $\ell$, and interacting with a LFL via two AFM Kondo couplings of equal strengths. The two impurities are coupled by the Heisenberg-type RKKY interaction $\lambda_{\mathrm{RKKY}} S_{\mathrm{I}}^{A} S_{\mathrm{II}}^{A} \cdot{ }^{4}$ The two-impurity Kondo problem has only been solved for certain values of $N, k$ and $\rho_{\mathrm{UV}}$.

At low energy or equivalently at large distances where $\ell$ is negligible, a CFT description of the two-impurity Kondo model becomes reliable [228,229]. The CFT approach begins with spatial averages over the momentum directions of the LFL fermion wave function, leading to a $(1+1)$-dimensional description, analogous to the $s$-wave reduction in the single-impurity Kondo model [228, 229]. However, now two modes per channel participate in the interactions, namely modes with even and odd parity about the mid-plane between the two impurities, leading to an effective doubling of the number of channels in $(1+1)$ dimensions from $k$ to $K=2 k$. In the IR, the difference in the Kondo couplings of even and odd channels become irrelevant [228, 229]. The CFT description thus involves $K$ channels of $(1+1)$-dimensional left-moving fermions interacting with identical Kondo couplings to two identical impurity spins at the origin.

The original two-impurity Kondo model with $N=2, K=2$, and $\rho_{\mathrm{UV}}$ the fundamental representation of $S U(2)$, has been studied using a combination of numerical RG [220-222, 227, 235, 236] and CFT techniques [227-232]. The results conform to intuition. In the FM RKKY limit, $\lambda_{\mathrm{RKKY}} / T_{\mathrm{K}} \rightarrow-\infty$, the two impurities lock into the triplet of $S U(2)$, in order to minimise the RKKY interaction. Upon lowering $T$, this effective spin-1 impurity is completely screened in a two-stage Kondo effect by the two fermion channels. The IR fixed point is a LFL with a $\pi / 2$ phase shift. In the AFM RKKY limit, $\lambda_{\mathrm{RKKY}} / T_{\mathrm{K}} \rightarrow+\infty$, the two impurities lock into the singlet of $S U(2)$ and effectively disappear from the spectrum. Consequently, no impurity remains that could be screened by the LFL, so the IR fixed point is a LFL with no phase shift. At $T=0$ particle-hole symmetry allows only two values of the phase shift, $\pi / 2$ and zero, so the FM and AFM RKKY limits must be separated by a quantum phase transition

\footnotetext{
${ }^{4}$ Strictly speaking, the term 'RKKY interaction' only refers to a Heisenberg interaction that is induced by Friedel oscillations in the LFL at order $\lambda_{\mathrm{K}}^{2}$ in perturbation theory $[181,219]$. In the large- $N$ limit, however, the RKKY interaction is sub-leading in $N$ and we need to add a Heisenberg interaction by hand [224]. In a (standard) abuse of terminology we will still call it RKKY coupling.
} 
where the phase shift jumps discontinuously [225, 229]. Numerical RG and CFT techniques show that the transition occurs at a non-zero $\mathrm{AFM}$ value $\lambda_{\mathrm{RKKY}} / T_{\mathrm{K}} \approx 2.2$ and is second-order and hence gives rise to a quantum critical point $[221,222,227,235,236]$. However, no change of symmetry occurs at the critical point: the ground state on both sides of the transition is a singlet of $S U(2) .{ }^{5}$

Although we lack a complete solution for general $N, k$ and $\rho_{\mathrm{UV}}$, the results for the original two-impurity Kondo problem suggests the following intuition for the general case. In the limit of infinitely strong FM RKKY coupling, $\lambda_{\mathrm{RKKY}} / T_{\mathrm{K}} \rightarrow-\infty$, or AFM RKKY coupling, $\lambda_{\mathrm{RKKY}} / T_{\mathrm{K}} \rightarrow+\infty$, the ground state should be an eigenstate of $S_{\mathrm{I}}^{A} S_{\mathrm{II}}^{A}$ with maximum or minimum eigenvalue, respectively. We shall denote the corresponding $S U(N)$ representations by $\rho_{\mathrm{FM}}$ and $\rho_{\mathrm{AFM}}$, respectively. For general values of $\lambda_{\mathrm{RKKY}} / T_{\mathrm{K}}$, the ground state will be a superposition of the eigenstates of $S_{\mathrm{I}}^{A} S_{\mathrm{II}}^{A}$ that appear in the tensor product $\rho_{\mathrm{UV}} \otimes \rho_{\mathrm{UV}}$. In the AFM or FM RKKY limits, $\lambda_{\mathrm{RKKY}} / T_{\mathrm{K}} \rightarrow \pm \infty$, the system effectively reduces to a $K$-channel $S U(N)$ Kondo model with a single impurity in a representation $\rho_{\mathrm{AFM}}$ or $\rho_{\mathrm{FM}}$, respectively. In the AFM case, in some special cases $\rho_{\mathrm{UV}}$ is such that the two impurity spins can lock into a singlet. In those cases, no Kondo screening will occur, and the IR fixed point will be a LFL with no phase shift. On the other hand, in the more general case that $\rho_{\mathrm{AFM}}$ is non-trivial the residual impurity spin will be Kondo screened to the extent possible by the $K$ channels. The coexistence of inter-impurity and Kondo screening is thus generic in the AFM limit. The AFM IR fixed point will then be either a non-LFL (overscreening) or a phase-shifted LFL (under- or critical-screening), depending on the values of $N, K$ and $\rho_{\mathrm{AFM}}$. By contrast, in the FM case, $\rho_{\mathrm{FM}}$ is always non-trivial: Kondo screening will occur and the IR fixed point will again be either a non-LFL or a phase-shifted LFL. In the special case that the IR fixed point in the AFM limit does not have a phase shift while that in the FM limit does, the two must be separated by a quantum phase transition [225], while in the more general case that both limits have phase shifts the evolution from one limit to the other may or may not be continuous.

\footnotetext{
${ }^{5}$ Surprisingly, numerical RG techniques reveal that the spin-spin correlator $\left\langle S_{\mathrm{I}}^{A} S_{\mathrm{II}}^{A}\right\rangle$ decreases smoothly and monotonically as $\lambda_{\mathrm{RKKY}} / T_{\mathrm{K}}$ increases from the FM limit, $\lambda_{\mathrm{RKKY}} / T_{\mathrm{K}} \rightarrow-\infty$, where $\left\langle S_{\mathrm{I}}^{A} S_{\mathrm{II}}^{A}\right\rangle=1 / 4$, the triplet value, to the AFM RKKY limit $\lambda_{\mathrm{RKKY}} / T_{\mathrm{K}} \rightarrow+\infty$, where $\left\langle S_{\mathrm{I}}^{A} S_{\mathrm{II}}^{A}\right\rangle=-3 / 4$, the singlet value $[221,222,227,236]$.
} 
Large- $N$ results for the two-impurity Kondo problem with $K=2$ channels appear in refs. [206, 224, 225]. The authors of refs. [224, 225] carefully chose a totally antisymmetric $\rho_{\mathrm{UV}}$ whose Young tableau had exactly $q=N / 2$ boxes to ensure that the two spins can lock into a singlet. Indeed, their large- $N$ saddle-point solution reveals a first-order quantum phase transition between an AFM phase with no phase shift and a FM phase with a $\pi / 2$ phase shift, indicating Kondo screening.

Our holographic model will also contain two totally anti-symmetric $S U(N)$ spin impurities. However, it will be too crude to allow us to identify the exact number of boxes $q$ in the corresponding Young tableau. We will only know that $q \propto N$. As a result, the AFM ground state will typically not be a singlet of $S U(N)$ and hence Kondo screening and a phase shift will occur. The coexistence of Kondo and inter-impurity screening is also widely believed to occur in the Kondo lattice [184, 185, 236].

As in the single-impurity case, the Abrikosov pseudo-fermion representation allows us to write the Kondo couplings of the two impurity spins as double-trace couplings with respect to $S U(N)$. It also allows us to write the RKKY coupling as a double-trace coupling of $S U(N)$, as we will now describe. To our knowledge the following results are novel. We introduce two species of pseudo-fermions, one for each spin:

$$
S_{i}^{A}=\chi_{i \alpha}^{\dagger} T_{\alpha \beta}^{A} \chi_{i \beta}, \quad i=\mathrm{I}, \mathrm{II}
$$

We can then define $\mathcal{O}_{\mathrm{I}} \equiv \psi_{\alpha}^{\dagger} \chi_{\mathrm{I} \alpha}$, which in the large- $N$ limit produces double-trace Kondo couplings of the form $-\lambda_{\mathrm{K}}^{\mathrm{I}} \mathcal{O}_{\mathrm{I}}^{\dagger} \mathcal{O}_{\mathrm{I}}$, and similarly for $\mathcal{O}_{\mathrm{II}}$ and $\lambda_{\mathrm{K}}^{\mathrm{II}}$. In our holographic model, we will always take $\lambda_{\mathrm{K}}^{\mathrm{I}}=\lambda_{\mathrm{K}}^{\mathrm{II}}=\lambda_{\mathrm{K}}$, following the CFT approach, in which the difference $\lambda_{\mathrm{K}}^{\mathrm{I}}-\lambda_{\mathrm{K}}^{\mathrm{II}}$ is irrelevant in the IR [228,229]. Generically, pseudo-fermions introduce an auxiliary $U(1)$ at each impurity site: each $U(1)$ acts by shifting the phase of the pseudo-fermions at that site. However, if the impurities are coincident and the RKKY coupling vanishes, then the auxiliary $U(1) \times U(1)$ is enhanced to $U(2)$, under which $\chi_{\mathrm{I} \alpha}$ and $\chi_{\mathrm{II} \alpha}$ combine into a doublet. The two scalars $\mathcal{O}_{\mathrm{I}}$ and $\mathcal{O}_{\mathrm{II}}$ thus also combine into a doublet of that $U(2), \mathcal{O} \equiv\left(\mathcal{O}_{\mathrm{I}}, \mathcal{O}_{\text {II }}\right)^{\mathrm{T}}$. We use $U(2)$ generators

$$
\tau^{b}=\frac{1}{2}\left(\mathbb{1}, \sigma^{1}, \sigma^{2}, \sigma^{3}\right), \quad b=0, \ldots, 3,
$$


with $\sigma^{1}, \sigma^{2}$, and $\sigma^{3}$ the Pauli matrices. The components of the auxiliary $U(2)$ Noether charges are then

$$
R^{b} \equiv \chi_{i \alpha}^{\dagger} \tau_{i j}^{b} \chi_{j \alpha}
$$

which obey the $(0+1)$-dimensional conservation equation $\partial_{t} R^{b}=0$. The constraint (5.3) on the auxiliary charge in the single-impurity case is generalised in the two-impurity case to constraints on the elements of $R^{b}$ in the Cartan of the auxiliary $U(2)$ : if $S_{\mathrm{I}}^{A}$ and $S_{\mathrm{II}}^{A}$ have $q_{\mathrm{I}}$ and $q_{\mathrm{II}}$ boxes in their Young tableaux, respectively, we must impose

$$
R^{0}=\frac{1}{2}\left(q_{\mathrm{I}}+q_{\mathrm{II}}\right), \quad R^{3}=\frac{1}{2}\left(q_{\mathrm{I}}-q_{\mathrm{II}}\right) .
$$

If the two impurities are identical, $q_{\mathrm{I}}=q_{\mathrm{II}}=q$, we have $R^{0}=q$ and $R^{3}=0$. Using the completeness relation in eq. (5.4) and $\chi_{i \alpha}$ 's anti-commutation relations, the RKKY interaction can be recast as a double-trace interaction with respect to $S U(N)$,

$$
\lambda_{\mathrm{RKKY}} S_{\mathrm{I}}^{A} S_{\mathrm{II}}^{A}=-\frac{1}{2} \lambda_{\mathrm{RKKY}}\left(\left(R^{1}\right)^{2}+\left(R^{2}\right)^{2}-\frac{1}{2}\left(q_{\mathrm{I}}+q_{\mathrm{II}}\right)+\frac{q_{\mathrm{I}} q_{\mathrm{II}}}{N}\right) .
$$

Upon dropping the insignificant constants $\left(q_{\mathrm{I}}+q_{\mathrm{II}}\right) / 2$ and $q_{\mathrm{I}} q_{\mathrm{II}} / N$, we thus find that the RKKY interaction is a classically-relevant double-trace interaction, of the form $-\lambda_{\text {RKKY }}\left(\left(R^{1}\right)^{2}+\left(R^{2}\right)^{2}\right)$. It explicitly breaks the auxiliary $U(2)$ symmetry down to the subgroup that commutes with $\left(R^{1}\right)^{2}+\left(R^{2}\right)^{2}$, namely to the Cartan of $U(2)$. The doubletrace form of the RKKY interaction will be extremely useful for our holographic model: a double-trace interaction will be realised holographically by a boundary condition on the $U(2)$ YM gauge field dual to $R^{b}$, as we will discuss in section 5.4.

To summarise: At large distances compared to $\ell$ and at large $N$, the two-impurity Kondo model reduces to a $(1+1)$-dimensional chiral CFT consisting of $K$ channels of left-moving fermions with one marginally-relevant, double-trace Kondo coupling for each impurity spin and a relevant, double-trace RKKY coupling between the impurity spins. As in the single-impurity case, we expect the Kondo effect to appear as condensation of $\mathcal{O}_{\text {I }}$ and $\mathcal{O}_{\text {II }}$ below some critical temperature. Via large- $N$ factorisation, $\left\langle S_{\mathrm{I}}^{A} S_{\mathrm{II}}^{A}\right\rangle \propto-\left\langle\left(R^{1}\right)^{2}+\left(R^{2}\right)^{2}\right\rangle \propto-\left\langle R^{1}\right\rangle^{2}-\left\langle R^{2}\right\rangle^{2}$, so we expect non-zero spin-spin correlations, $\left\langle S_{\mathrm{I}}^{A} S_{\mathrm{II}}^{A}\right\rangle \neq 0$, to appear as condensation of $R^{1}$ and/or $R^{2}$. Both effects will indeed appear in our holographic model. 


\subsection{A holographic two-impurity model}

In this section we present a holographic two-impurity Kondo model, extending the single-impurity model from ref. [18]. In order to reach a holographic description of the Kondo model, we need to introduce additional degrees of freedom in the adjoint representation of the $S U(N)$ spin symmetry, including in particular $S U(N)$ gauge fields. This introduces an additional coupling besides the Kondo and RKKY couplings, namely the 't Hooft coupling. We then take the 't Hooft large- $N$ limit and the limit of large 't Hooft coupling. We choose the adjoint degrees of freedom such that, in these limits, we obtain a CFT holographically dual to Einstein-Hilbert gravity in $A d S_{3}$. For a specific example of such a construction, see ref. [18]. Effectively, our holographic model replaces the free chiral fermions in the UV with a strongly coupled CFT.

Following ref. [18], we will work with a 'bottom-up' model, built from the minimal ingredients that must be present in any holographic Kondo model, but with enough structure to describe the essential phenomena. Each $S U(N)$-invariant, single-trace, operator with dimension of order $N^{0}$ is holographically dual to a field in the gravity description. The stress-energy tensor of the $(1+1)$-dimensional CFT is dual to the metric in $A d S_{3}$. The channel and electromagnetic $S U(K)_{N} \times U(1)$ currents of the chiral fermions are dual to a $S U(K)_{N} \times U(1)$ Chern-Simons gauge field [270], which in form notation we call $A$. The auxiliary $U(2)$ charges $R^{b}$ at the impurities' location $x=0$ are dual to a $U(2)$ YM gauge field, which in form notation we call $a=a^{b} \tau^{b}$, localised to the $A d S_{2}$ subspace at $x=0$. The complex scalar $\mathcal{O}=\left(\mathcal{O}_{\mathrm{I}}, \mathcal{O}_{\mathrm{II}}\right)^{\mathrm{T}}$ at the impurities' location is bi-fundamental under $S U(K)_{N} \times U(1)$ and the auxiliary $U(2)$, and is dual to a complex scalar field $\Phi=\left(\Phi_{\mathrm{I}}, \Phi_{\mathrm{II}}\right)^{\mathrm{T}}$, localised to the $A d S_{2}$ subspace and bi-fundamental under the $S U(K)_{N} \times U(1)$ Chern-Simons and $U(2)$ YM gauge fields. ${ }^{6}$

\footnotetext{
${ }^{6}$ Our choices of spin and charge for $\mathcal{O}$ indicate unambiguously that our model describes a Kondo rather than an Anderson model, and that the impurity spins are in totally anti-symmetric representations of $S U(N)$. Totally symmetric representations, for example, would involve Schwinger bosons rather than Abrikosov pseudo-fermions [208,268,269], in which case $\mathcal{O}$ would be fermionic. In the Anderson model, the impurity is a bi-linear of two physical $f$ electrons which are charged under the $U(1)$ of electromagnetism, in contrast to the pseudo-fermions which are neutral under that $U(1)$ [181]. The Anderson model would thus require a complex scalar similar to our $\mathcal{O}$, but built from a chiral fermion $\psi$ and the $f$ electron and hence neutral under the electric $U(1)$.
} 
We will work in a probe limit, i.e. when $N \rightarrow \infty$ we keep $K$ fixed and compute all expectation values only to order $N$. In our holographic model, that means the EinsteinHilbert action will scale as $N^{2}$, but the matter action will scale as $N$. The matter fields' contribution to the Einstein equation can then be neglected in the large- $N$ limit and we only need to solve the matter fields' equations of motion in a fixed background metric. To describe a $(1+1)$-dimensional $\mathrm{CFT}$ on the real line with non-zero $T$, we must use the $A d S_{3}$-Schwarzschild, or BTZ, black brane metric,

$$
d s_{\mathrm{BTZ}}^{2}=\frac{1}{z^{2}}\left(\frac{1}{h(z)} \mathrm{d} z^{2}-h(z) \mathrm{d} t^{2}+\mathrm{d} x^{2}\right), \quad h(z)=1-\frac{z^{2}}{z_{H}^{2}},
$$

where $z$ is the holographic radial coordinate, with the boundary at $z=0$ and the horizon at $z=z_{H}$, while $t$ and $x$ are the CFT time and space directions. We have chosen units in which the $A d S_{3}$ radius is unity. The Hawking temperature of the black brane, $T=1 /\left(2 \pi z_{H}\right)$, is equal to the temperature of the dual CFT. The impurity is located at the $x=0$ subspace with induced metric

$$
g_{m n} \mathrm{~d} x^{m} x^{n}=\frac{1}{z^{2}}\left(\frac{1}{h(z)} \mathrm{d} z^{2}-h(z) \mathrm{d} t^{2}\right), \quad m, n=z, t .
$$

For simplicity, we henceforth take $K=1$. In that case, $S U(K)_{N} \times U(1)$ reduces to $U(1)$, so our Chern-Simons gauge field $A$ is Abelian with field strength $F=\mathrm{d} A$. For a discussion of the generalisation to $K>1$ see the end of section 3 in ref. [15].

Following ref. [18] we choose the simplest two-derivative action $S$ quadratic in the fields for our holographic two-impurity Kondo model. The action splits into two terms, one for the Chern-Simons gauge field, $S_{\mathrm{CS}}$, and one for the fields $a$ and $\Phi$ in the $A d S_{2}$ subspace, $S_{A d S_{2}}$,

$$
\begin{gathered}
S=S_{\mathrm{CS}}+S_{A d S_{2}}, \\
S_{\mathrm{CS}}=-\frac{N}{4 \pi} \int_{\mathrm{BTZ}} A \wedge \mathrm{d} A, \\
S_{A d S_{2}}=-N \int_{x=0} \mathrm{~d} z \mathrm{~d} t \sqrt{-g}\left[\frac{1}{2} \operatorname{tr}\left(f^{m n} f_{m n}\right)+\left(D^{m} \Phi\right)^{\dagger}\left(D_{m} \Phi\right)+M^{2} \Phi^{\dagger} \Phi\right],
\end{gathered}
$$

where $f_{m n}$ is the field strength of the $A d S_{2}$ YM field, while $D_{m}$ is the $U(2)$ gaugecovariant derivative, which acts on $f_{m n}$ and $\Phi$ as

$$
D_{m} f^{n p}=\nabla_{m} f^{n p}-i\left[a_{m}, f^{n p}\right], \quad D_{m} \Phi=\left(\partial_{m}+i A_{m}-i a_{m}\right) \Phi .
$$


We will fix the value of $\Phi$ 's mass-squared $M^{2}$ in section 5.4 . We will see in section 5.5 that the action in eq. (5.13) is sufficient to capture the basic physics of the large- $N$ twoimpurity Kondo model and can thus serve as a foundation for further model-building, for example by adding terms higher-order in derivatives or in the fields.

If we define the $U(2)$ gauge current

$$
J_{m}^{b} \equiv-i\left(\Phi^{\dagger} \tau^{b}\left(D_{m} \Phi\right)-\left(D_{m} \Phi\right)^{\dagger} \tau^{b} \Phi\right)
$$

then the equations of motion that follow from the action in eq. (5.13) are

$$
\begin{gathered}
\epsilon^{n \mu \nu} F_{\mu \nu}=-8 \pi \delta(x) \sqrt{-g} g^{n m} J_{m}^{0}, \quad F_{z t}=0, \\
\left(D_{m} f^{m n}\right)^{b}=-g^{n m} J_{m}^{b}, \\
\left(D_{m} D^{m}-M^{2}\right) \Phi=0,
\end{gathered}
$$

where $\mu, \nu=z, t, x$, and we choose $(z, t, x)$ to be a right-handed coordinate chart, $\epsilon^{z t x}=1$. We will work in radial gauge for both gauge fields, $A_{z}=0$ and $a_{z}^{b}=0$.

We recover the single-impurity case from ref. [18] if we only retain the $U(1)$ component $a_{m}^{0}$ of the YM field, and correspondingly only a single component of the scalar doublet. We will discuss correlators in the single-impurity case in section 5.6.

In the two-impurity case, discussed in sections 5.4 and 5.5, we will restrict ourselves to an investigation of the phase diagram. This means that we are only concerned with time-independent solutions, for which the equations (5.16a) for the Chern-Simons field $A$ simplify to

$$
\begin{gathered}
\partial_{x} A_{t}=4 \pi \delta(x) \sqrt{-g} g^{z z} J_{z}^{0}, \\
\partial_{z} A_{x}=4 \pi \delta(x) \sqrt{-g} g^{t t} J_{t}^{0}, \\
\partial_{z} A_{t}=0
\end{gathered}
$$

while the equation (5.16b) for the $U(2)$ YM field $a$ simplifies to $J_{z}^{0}=0$, plus a constraint (first order in derivatives)

$$
\epsilon^{b c d} g^{t t} a_{t}^{c} \partial_{z} a_{t}^{d}=J_{z}^{b}, \quad b, c, d=1,2,3,
$$


and a dynamical equation (second order in derivatives)

$$
\frac{1}{\sqrt{-g}} \partial_{z}\left(\sqrt{-g} g^{z z} g^{t t} \partial_{z} a_{t}^{b}\right)=-g^{t t} J_{t}^{b}
$$

Eqs. (5.17a) and (5.17c) together with $J_{z}^{0}=0$ imply that $A_{t}$ is a constant. Regularity requires $A_{t}=0$ to vanish at $z=z_{H}$, hence $A_{t}=0$ everywhere. The only remaining non-trivial component of the Chern-Simons field is then $A_{x}$, which does not appear in the equations of motion for $a$ and $\Phi$. In particular, eq. (5.16c) simplifies to

$$
\frac{1}{\sqrt{-g}} \partial_{z}\left(\sqrt{-g} g^{z z} \partial_{z} \Phi\right)-\left(M^{2}+g^{t t} a_{t}^{b} a_{t}^{c} \tau^{b} \tau^{c}\right) \Phi=0 .
$$

We can thus solve for $a$ and $\Phi$, and then plug those solutions into eq. (5.17b) to find $A_{x}$. However, we will not present explicit solutions for $A_{x}$ in the following. We will only need to know that non-trivial solutions for $A_{x}$ exist.

\subsection{Kondo and RKKY couplings from boundary con- ditions}

In this section we present the holographic renormalisation [53, 271-273] of our holographic two-impurity Kondo model. This will allow us to identify the Kondo and RKKY couplings in our model and to compute the free energy, which we will use to study the phase diagram of our model in section 5.5.

In our case, holographic renormalisation is non-trivial because our model includes a gauge field $a_{m}^{b}$ in an $A d S_{2}$ subspace. As is well-known (see e.g. [18, 274,275]), a solution of the YM equations in $A d S_{2}$ typically diverges near the boundary, in contrast to YM gauge fields in higher-dimensional AdS spacetimes. Indeed, solving eqs. (5.18) and (5.19) around the $A d S_{2}$ boundary, $z=0$, we find $a_{t}^{b}=Q^{b} / z+\ldots$, where ... denotes terms sub-leading as $z \rightarrow 0$. The constants $Q^{b}$ are the fluxes at the boundary,

$$
\lim _{z \rightarrow 0} \star f^{b}=\lim _{z \rightarrow 0} \sqrt{-g} g^{z z} g^{t t} f_{z t}^{b}=Q^{b}
$$

with $\star$ the Hodge star of $A d S_{2}$. The fluxes $Q^{b}$ determine the expectation values of the conserved $U(2)$ charges $R^{b}$. If our model was top-down, we could in principle derive an exact relation between $Q^{b}$ and $R^{b}$. However, in our bottom-up model we can only 
assume that the relation between the two is monotonic. As discussed below eq. (5.10), two identical impurity spins, each in a totally anti-symmetric representation with $q$ boxes, must obey $R^{0}=q$ and $R^{3}=0$. In our holographic model, we will therefore consider various values of $Q^{0}$, but will always take $Q^{3}=0 .^{7}$

Although the leading solution $Q^{b} / z$ diverges as $z \rightarrow 0$, it is nevertheless normalisable according to the criteria of refs. [72,276], as shown in the appendix of ref. [15]. Even so, the divergence of $Q^{b} / z$ can affect the asymptotics of other fields coupled to $a_{m}^{b}$ such as our charged $\Phi$. In $\Phi$ 's equation of motion (5.20) the coupling to the YM field asymptotically approaches a constant, $\lim _{z \rightarrow 0} g^{t t} a_{t}^{b} a_{t}^{c}=-Q^{b} Q^{c}$, which is the same order in $z$ as $M^{2}$. The YM field thus effectively shifts $\Phi$ 's mass-squared matrix from $M^{2}$ times the $U(2)$ identity matrix to $M^{2}-Q^{b} Q^{c} \tau^{b} \tau^{c}$. The powers of $z$ that appear in $\Phi$ 's asymptotic expansion will thus be determined by $M^{2}-Q^{b} Q^{c} \tau^{b} \tau^{c}$. Those powers determine the dimension of $\Phi$ 's dual operator $\mathcal{O}$ at the UV fixed point. As a result, fixing $M^{2}$ and changing the $Q^{b}$ will change $\mathcal{O}$ 's UV dimension and thus change the UV fixed point. This does not happen in the original two-impurity Kondo model, where $\mathcal{O}$ 's UV dimension is always $1 / 2$, regardless of the choice of $R^{b}$ (or equivalently of $\rho_{\mathrm{UV}}$ ). In other words, this is a special feature of the holographic model, which by process of elimination must be due to the additional, strongly interacting degrees of freedom we introduced. The same effect appeared in the holographic single-impurity Kondo model of ref. [18] and the holographic Bose-Hubbard model of ref. [275].

However, a theory in asymptotically AdS requires a well-defined boundary value problem, which means that we must fix the asymptotics of all fields. We will therefore take an unusual step: when $Q^{b}$ changes we will change $M^{2}$ in order to maintain $\Phi$ 's asymptotics. Specifically, we will demand that $\mathcal{O}$ always has dimension $1 / 2$ in the UV, so that the Kondo couplings in our model are always classically marginal. Something similar was done in the holographic single-impurity Kondo model of ref. [18].

We fix $\Phi$ 's asymptotics as follows. We diagonalise $\Phi$ 's mass matrix,

$$
M^{2}-Q^{b} Q^{c} \tau^{b} \tau^{c}=\mathcal{S}\left(\begin{array}{cc}
M_{-}^{2} & \\
& M_{+}^{2}
\end{array}\right) \mathcal{S}^{\dagger}
$$

\footnotetext{
${ }^{7}$ Our choice $K=1$ then guarantees, based on $S U(N)$ representation theory arguments and Pauli exclusion alone, that overscreening cannot occur in our model [209].
} 
using the unitary matrix

$$
\mathcal{S}=\frac{1}{\sqrt{2}}\left(\begin{array}{cc}
\operatorname{sgn}\left(Q^{0}\right) \frac{Q^{1}-i Q^{2}}{\sqrt{\left(Q^{1}\right)^{2}+\left(Q^{2}\right)^{2}}} & -\operatorname{sgn}\left(Q^{0}\right) \frac{Q^{1}-i Q^{2}}{\sqrt{\left(Q^{1}\right)^{2}+\left(Q^{2}\right)^{2}}} \\
1 & 1
\end{array}\right),
$$

and eigenvalues

$$
M_{\mp}^{2}=M^{2}-\frac{1}{4}\left(\left|Q^{0}\right| \pm \sqrt{\left(Q^{1}\right)^{2}+\left(Q^{2}\right)^{2}}\right)^{2} .
$$

The modes with these eigenvalues are the components $\phi_{-}$and $\phi_{+}$of $\mathcal{S}^{-1} \Phi \equiv\left(\phi_{-}, \phi_{+}\right)^{\mathrm{T}}$, whose asymptotic expansions are thus determined by $M_{-}^{2}$ and $M_{+}^{2}$ respectively.

Our Kondo interactions are of the form $\mathcal{O}_{\text {I }}^{\dagger} \mathcal{O}_{\text {I }}$ and $\mathcal{O}_{\text {II }}^{\dagger} \mathcal{O}_{\text {II }}$, where $\mathcal{O}_{\text {I }}$ and $\mathcal{O}_{\text {II }}$ are dual to $\Phi_{\mathrm{I}}$ and $\Phi_{\mathrm{II}}$, the components of $\Phi=\left(\Phi_{\mathrm{I}}, \Phi_{\mathrm{II}}\right)^{\mathrm{T}}$. To obtain classically marginal Kondo couplings we want both $\mathcal{O}_{\text {I }}$ and $\mathcal{O}_{\text {II }}$ to have dimension $1 / 2$. That requires $\Phi_{\text {I }}$ and $\Phi_{\text {II }}$ to have asymptotic powers of $z$ identical to those of a scalar field that saturates the BF bound (2.20), which in $A d S_{2}$ means leading asymptotic terms $\sqrt{z}$ and $\sqrt{z} \ln (z)$. The components $\Phi_{\mathrm{I}}$ and $\Phi_{\mathrm{II}}$ are linear combinations of $\phi_{-}$and $\phi_{+}$. We guarantee that $\Phi_{\mathrm{I}}$ and $\Phi_{\mathrm{II}}$ each has the asymptotics of a scalar at the BF bound as follows. First, we set $M_{-}^{2}$ to the $A d S_{2}$ BF bound, $M_{-}^{2}=-1 / 4$, which via eq. (5.24) fixes $M^{2}$ in terms of $Q^{0}$, $Q^{1}$, and $Q^{2}$. Second, we choose an ansatz in which $\phi_{+}$vanishes identically. ${ }^{8}$

Setting $\phi_{+}=0$ is only consistent if $\phi_{+}$is not sourced by other fields in its equation of motion, eq. (5.20) multiplied by $\mathcal{S}^{-1}$. That leads to three constraints. First, $a_{t}^{3}=0$, which is indeed a solution of $a_{t}^{3}$ 's equation of motion, eq. (5.19) with $b=3$, when $\phi_{+}=0$. Moreover, $a_{t}^{3}=0$ implies $Q^{3}=0$ as required for two identical impurities. Second, $a_{t}^{1}=\frac{Q^{1}}{Q^{2}} a_{t}^{2}$. However, eq. (5.19) implies that $\frac{Q^{1}}{Q^{2}} a_{t}^{2}$ satisfies $a_{t}^{1}$ 's equation of motion if and only if $Q^{1}=Q^{2}$. We therefore take $Q^{1}=Q^{2}$ and $a_{t}^{1}=a_{t}^{2}$. Third, $\operatorname{Re} \phi_{-} \propto \operatorname{Im} \phi_{-}$, which comes from the $U(2)$ constraint in eq. (5.18). Eqs. (5.19) and (5.20) then imply $\operatorname{Re} \phi_{-}= \pm \operatorname{Im} \phi_{-}$. We will choose $\operatorname{Re} \phi_{-}=\operatorname{Im} \phi_{-}$and define

$$
\phi \equiv \operatorname{Re} \phi_{-}=\operatorname{Im} \phi_{-} .
$$

In summary, our ansatz includes

$$
\mathcal{S}^{-1} \Phi \equiv \phi\left(\begin{array}{c}
1+i \\
0
\end{array}\right), \quad a_{t}^{3}=0, \quad a_{t}^{1}=a_{t}^{2} .
$$

\footnotetext{
${ }^{8}$ We do not set $M_{+}^{2}=-1 / 4$, because then $M_{-}^{2}$ would violate the BF bound, producing an instability.
} 
A non-trivial solution for $\phi$ breaks $U(2)$ down to a single $U(1)$ generated by $\tau^{0}-\tau^{3}$. We will discuss the symmetry breaking in our model in more detail in section 5.5.

For the ansatz in eq. (5.26), a straightforward exercise shows that $\phi=\left|\Phi_{\mathrm{I}}\right|=\left|\Phi_{\mathrm{II}}\right|$. Therefore, our two Kondo couplings will be equal, $\lambda_{\mathrm{I}}=\lambda_{\mathrm{II}}=\lambda_{\mathrm{K}}$, and the strengths of the Kondo screening clouds will be equal, $\left\langle\left|\mathcal{O}_{\mathrm{I}}\right|\right\rangle=\left\langle\left|\mathcal{O}_{\mathrm{II}}\right|\right\rangle$, as desired. The ansatz (5.26) simplifies the equations of motion dramatically. It is convenient to define a rescaled $a_{t}^{1}$,

$$
\mathcal{A}_{t} \equiv \sqrt{2} \operatorname{sgn}\left(Q^{0} Q^{1}\right) a_{t}^{1}
$$

which is holographically dual to

$$
\mathcal{R} \equiv \sqrt{2} \operatorname{sgn}\left(Q^{0} Q^{1}\right) R^{1}
$$

and which has an asymptotic expansion

$$
\mathcal{A}_{t}=\frac{\mathcal{Q}}{z}+\mu+\ldots, \quad \mathcal{Q} \equiv \sqrt{2} \operatorname{sgn}\left(Q^{0} Q^{1}\right) Q^{1}, \quad \mu \equiv \sqrt{2} \operatorname{sgn}\left(Q^{0} Q^{1}\right) \mu^{1} .
$$

We then define

$$
\mathcal{A}_{t}^{ \pm} \equiv \frac{1}{2}\left(a_{t}^{0} \pm \mathcal{A}_{t}\right)
$$

which are dual to $\mathcal{R}^{ \pm} \equiv \frac{1}{2}\left(R^{0} \pm \mathcal{R}\right)$ and which have the asymptotic expansions

$$
\mathcal{A}_{t}^{ \pm}=\frac{\mathcal{Q}^{ \pm}}{z}+\mu^{ \pm}+\ldots, \quad \mathcal{Q}^{ \pm}=\frac{1}{2}\left(Q^{0} \pm \mathcal{Q}\right) .
$$

Inserting $\mathcal{Q}^{+}$into eq. (5.24) we find that our choice $M_{-}^{2}=-1 / 4$ implies $M^{2}=-1 / 4+$ $\left(\mathcal{Q}^{+}\right)^{2}$. The equations of motion, eqs. (5.19) and (5.20), then reduce to

$$
\begin{gathered}
\partial_{z}\left(\sqrt{-g} g^{z z} g^{t t} \partial_{z} \mathcal{A}_{t}^{-}\right)=0, \\
\partial_{z}\left(\sqrt{-g} g^{z z} g^{t t} \partial_{z} \mathcal{A}_{t}^{+}\right)=\sqrt{-g} g^{t t} 2 \mathcal{A}_{t}^{+} \phi^{2}, \\
\partial_{z}\left(\sqrt{-g} g^{z z} \partial_{z} \phi\right)-\sqrt{-g}\left(M^{2}+g^{t t}\left(\mathcal{A}_{t}^{+}\right)^{2}\right) \phi=0 .
\end{gathered}
$$

Clearly $\mathcal{A}_{t}^{-}$decouples from $\mathcal{A}_{t}^{+}$and $\phi$, and is trivial to solve for: $\mathcal{A}_{t}^{-}=\mathcal{Q}^{-} / z+\mu^{-}$. On the other hand, $\mathcal{A}_{t}^{+}$and $\phi$ remain coupled and we have been able to solve their equations of motion (5.32b) and (5.32c) only numerically, as we will discuss in section 5.5. ${ }^{9}$

\footnotetext{
${ }^{9}$ Eqs. (5.32b) and (5.32c) are actually identical in form to the equations of motion in the holographic single-impurity Kondo model of ref. [18], but where ref. [18] had $a_{t}^{0}$ and $\phi$ we have $\mathcal{A}_{t}^{+}$and $\phi$. However, we will see that the boundary conditions on $\mathcal{A}_{t}^{+}$and $\phi$ in our two-impurity model are very different from those in ref. [18] and that they will effectively couple $\mathcal{A}_{t}^{-}$to $\mathcal{A}_{t}^{+}$and $\phi$.
} 


\subsubsection{Holographic renormalisation}

In order to renormalise our model we switch to a Fefferman-Graham gauge [?,271,277] with radial coordinate

$$
r=\ln \left(\frac{1+\sqrt{1-z^{2} / z_{H}^{2}}}{2 z}\right),
$$

in terms of which the $A d S_{2}$ defect (5.12) takes the form

$$
g_{m n} d x^{m} d x^{n}=d r^{2}+\gamma(r) d t^{2}
$$

At the $A d S_{2}$ boundary $r \rightarrow \infty, r$ is related to $z$ as $r=-\ln (z)+\ldots$, and $\gamma(r)$ diverges as $-e^{2 r}$. The asymptotic solutions of the equations of motion (5.32a), (5.32b) and (5.32c) for our choice $M^{2}=-1 / 4+\left(\mathcal{Q}^{+}\right)^{2}$ are

$$
\begin{aligned}
\mathcal{A}_{t}^{-}= & e^{r} \mathcal{Q}^{-}+\mu^{-}+\ldots, \\
\mathcal{A}_{t}^{+}= & e^{r} \mathcal{Q}^{+}-2 \mathcal{Q}^{+}\left(\frac{1}{3} \alpha^{2} r^{3}+\left(\alpha^{2}-\alpha \beta\right) r^{2}+\left(2 \alpha^{2}-2 \alpha \beta+\beta^{2}\right) r\right) \\
& +\mu^{+}+\ldots, \\
& +e^{-r / 2}(-\alpha r+\beta)+\ldots,
\end{aligned}
$$

where $\alpha, \beta, \mathcal{Q}^{-}$, and $\mu^{ \pm}$are integration constants.

We determine the required counterterms as follows. We first produce a regulated action $S_{\text {reg }}(r)$ by integrating only up to a large but finite value of $r$. We then identify the diverging terms by asymptotically solving the radial Hamilton-Jacobi (HJ) equation for the regulated on-shell action $S_{\text {reg }}(r)$ [64,66,273,278], using the covariant ansatz

$$
S_{\mathrm{reg}}=2 N \int \mathrm{d} t \sqrt{-\gamma}\left(F(u, v)-u-\frac{1}{2} \gamma^{-1}\left(\mathcal{A}_{t}^{-}\right)^{2}\right), u \equiv \frac{1}{2} \gamma^{-1}\left(\mathcal{A}_{t}^{+}\right)^{2}, \quad v \equiv \phi^{2}
$$

As mentioned above, we need $M^{2}-\left(\mathcal{Q}^{+}\right)^{2}=-1 / 4$ in order for $\phi$ 's asymptotic behaviour to be well-defined. This implies that $u$ satisfies a constraint asymptotically as $r \rightarrow \infty$,

$$
2 u+M^{2}+\frac{1}{4}=\mathcal{O}\left(r^{3} e^{-r}\right) .
$$

Ref. [279] showed that in this case the solution $F(u, v)$ to the HJ equation takes the form of a Taylor-expansion in the constraint,

$$
F(u, v)=\sum_{k \geq 0} f_{k}(v)\left(u+\frac{M^{2}+1 / 4}{2}\right)^{k} .
$$


While only the $k=0$ term contributes to the divergences of the on-shell action, we must keep all terms up to and including order $k$ to renormalise correlators with $k$ insertions of the operator dual to $\mathcal{A}_{t}^{+}$[17]. We will only consider correlators involving at most one insertion of this operator so that we will only need to determine $f_{0}(v)$ and $f_{1}(v)$. Asymptotically solving the HJ equation then yields the required counterterms:

$$
\begin{aligned}
S_{\mathrm{ct}}=-2 N \int \mathrm{d} t \sqrt{-\gamma}\{ & \phi^{2}\left(\frac{1}{2}-\frac{1}{r}\right)-\frac{1}{2} \gamma^{-1}\left(\mathcal{A}_{t}^{+}\right)^{2}-\frac{1}{2} \gamma^{-1}\left(\mathcal{A}_{t}^{-}\right)^{2} \\
& \left.-\frac{2}{3} \phi^{2}\left(r-\frac{\beta}{\alpha}\right)\left(\frac{1}{2} \gamma^{-1}\left(\mathcal{A}_{t}^{+}\right)^{2}+\frac{M^{2}+1 / 4}{2}\right)\right\} .
\end{aligned}
$$

We refer the reader to refs. [15,17] for many more details on the renormalisation of our model. The explicit dependence on the cutoff $r$ in eq. (5.39) reflects the conformal anomaly induced by the scalar operator at the BF bound $[60,160,280]$. The counterterms also explicitly depend on the ratio $\beta / \alpha$ of $\phi$ 's asymptotic coefficients. We will shortly show that $\beta / \alpha$ is held fixed by $\phi$ 's boundary condition so that this counterterm is well-defined. ${ }^{10}$ We can now define the subtracted and renormalised actions as $S_{\text {sub }}(r) \equiv S_{\text {reg }}+S_{\text {ct }}$ and $S_{\text {ren }} \equiv \lim _{r \rightarrow \infty} S_{\text {sub }}$. The renormalised one-point functions for Dirichlet boundary conditions are then

$$
\begin{aligned}
& \frac{\delta S_{\text {ren }}}{\delta \alpha} \equiv \lim _{r \rightarrow \infty}\left[-\frac{e^{r / 2} r}{\sqrt{-\gamma}} \frac{\delta S_{\text {sub }}}{\delta \phi}\right]=-4 N \beta, \\
& \frac{\delta S_{\text {ren }}}{\delta Q^{0}} \equiv \lim _{r \rightarrow \infty}\left[\frac{e^{2 r}}{\sqrt{-\gamma}} \frac{\delta S_{\text {sub }}}{\delta a_{t}^{0}}\right]=-N \mu^{0}-2 N \mathcal{Q}^{+}\left(2 \alpha^{2}-2 \alpha \beta+\beta^{2}-\frac{1}{3} \frac{\beta^{3}}{\alpha}\right), \\
& \frac{\delta S_{\text {ren }}}{\delta \mathcal{Q}} \equiv \lim _{r \rightarrow \infty}\left[\frac{e^{2 r}}{\sqrt{-\gamma}} \frac{\delta S_{\text {sub }}}{\delta \mathcal{A}_{t}}\right]=-N \mu-2 N \mathcal{Q}^{+}\left(2 \alpha^{2}-2 \alpha \beta+\beta^{2}-\frac{1}{3} \frac{\beta^{3}}{\alpha}\right),
\end{aligned}
$$

where we used $\mathcal{A}_{t}^{ \pm}=\frac{1}{2}\left(a_{t}^{0} \pm \mathcal{A}_{t}\right), \mathcal{Q}^{ \pm}=\frac{1}{2}\left(Q^{0} \pm \mathcal{Q}\right)$, and $\mu^{ \pm}=\frac{1}{2}\left(\mu^{0} \pm \mu\right)$ from eqs. (5.29)-(5.31).

\subsubsection{Double-trace Kondo and RKKY couplings}

As discussed in section 5.2, introducing Abrikosov fermions allows us to write the Kondo and RKKY interactions as double-trace deformations of the Hamiltonian with respect

\footnotetext{
${ }^{10}$ Refs. [281, 282] observed the same in holographic counterterms for irrelevant operators: they depend on sub-leading modes, but are well-defined for boundary conditions dual to multi-trace couplings.
} 
to $S U(N)$ spin, eqs. (5.5) and (5.10). In the large- $N$ limit, they take the form

$$
\begin{gathered}
\mathcal{H}_{\mathrm{K}}=-\frac{1}{2} N \cdot N \lambda_{\mathrm{K}}\left(\frac{\mathcal{O}_{\mathrm{I}}^{\dagger} \mathcal{O}_{\mathrm{I}}}{N^{2}}+\frac{\mathcal{O}_{\mathrm{II}}^{\dagger} \mathcal{O}_{\mathrm{II}}}{N^{2}}\right), \\
\mathcal{H}_{\mathrm{RKKY}}=-\frac{1}{2} N \cdot N \lambda_{\mathrm{RKKY}}\left(\frac{\left(R^{1}\right)^{2}}{N^{2}}+\frac{\left(R^{2}\right)^{2}}{N^{2}}\right),
\end{gathered}
$$

where both impurities have equal Kondo couplings $\lambda_{\mathrm{K}}$. We keep $N \lambda_{\mathrm{K}}$ and $N \lambda_{\mathrm{RKKY}}$ fixed as $N \rightarrow \infty$ so that $\mathcal{H}_{\mathrm{K}}$ and $\mathcal{H}_{\mathrm{RKKY}}$ are $\mathcal{O}(N)$. Our ansatz (5.26) only allows us to describe states with $\mathcal{O}_{\text {I }}=\mathcal{O}_{\text {II }}$ and $R^{1}=R^{2}$. Within that subspace, eq. (5.43) becomes

$$
\mathcal{H}_{\mathrm{K}}=-N \cdot N \lambda_{\mathrm{K}} \frac{\left|\mathcal{O}_{\mathrm{I}}\right|^{2}}{N^{2}}, \quad \mathcal{H}_{\mathrm{RKKY}}=-\frac{1}{2} N \cdot N \lambda_{\mathrm{RKKY}} \frac{\mathcal{R}^{2}}{N^{2}},
$$

recalling from eq. (5.28) that $\mathcal{R}^{2}=2\left(R^{1}\right)^{2}=2\left(R^{2}\right)^{2}$.

Let us start with the marginally relevant Kondo interaction. To leading order in $N$, it simply adds to the generating functional the term

$$
S_{\mathrm{K}}=-N \cdot N \lambda_{K} \int \mathrm{d} t \frac{\left\langle\left|\mathcal{O}_{\mathrm{I}}\right|\right\rangle^{2}}{N^{2}}
$$

thanks to large- $N$ factorisation [283]. Such a deformation is therefore implemented holographically by adding $S_{\mathrm{K}}$ as a finite boundary term to the dual gravity action [283, 284]. Using from eq. (5.40) that $\left\langle\left|\mathcal{O}_{\mathrm{I}}\right|\right\rangle=-4 N \beta$, we can write $S_{\mathrm{K}}$ as

$$
S_{\mathrm{K}}=2 N \int \mathrm{d} t \kappa \beta^{2}, \quad \kappa \equiv-8 N \lambda_{\mathrm{K}}
$$

A variation of the generating functional in the deformed theory gives

$$
\delta\left(S_{\mathrm{ren}}+S_{\mathrm{K}}\right)=\int \mathrm{d} t(-4 N \beta) \delta(\alpha-\kappa \beta),
$$

showing that the expectation value $\left\langle\left|\mathcal{O}_{\mathrm{I}}\right|\right\rangle=-4 N \beta$ remains unchanged, while the source for $\left|\mathcal{O}_{\mathrm{I}}\right|$ changes as $\alpha \longrightarrow \alpha-\kappa \beta$. As a result, states that include the doubletrace Kondo deformation but no single-trace deformation will be described by bulk solutions for $\phi$ satisfying the boundary condition $\alpha=\kappa \beta$ with fixed $\kappa .^{11}$

\footnotetext{
${ }^{11}$ In the holographic single-impurity Kondo model of ref. [18], the finite boundary term involving the scalar field was different from our $S_{\mathrm{K}}$ : it had the same form as our $S_{\mathrm{K}}$, but with $\beta \rightarrow \alpha$ and $\kappa \rightarrow 1 / \kappa$. In that case, the linear combination of $\alpha$ and $\beta$ held fixed in the variational principle would not be $\alpha-\kappa \beta$. Nevertheless, $\alpha-\kappa \beta$ was held fixed in ref. [18]. Those two wrongs made a right, in the following sense: the finite boundary term in ref. [18], when evaluated on $\alpha=\kappa \beta$, actually agrees with our $S_{\mathrm{K}}$, so the solutions for the scalar and the value of the on-shell action of ref. [18] actually agree with those obtained using our $S_{\mathrm{K}}$. Similarly, the one-point function identified as $\langle\mathcal{O}\rangle \propto N \alpha$ in ref. [18] agrees with our $\langle\mathcal{O}\rangle \propto N \beta$ when evaluated on $\alpha=\kappa \beta$.
} 
Let us now turn to the relevant RKKY interaction. The charge $\mathcal{R}$ has conformal dimension zero, and hence its expectation value $\langle\mathcal{R}\rangle$ must be determined by $\mathcal{A}_{t}$ 's leading mode $\mathcal{Q}$ rather than by its sub-leading mode $\mu$. The generating functional for $\mathcal{R}$ must thus be given by the Legendre transform of $S_{\text {ren }}+S_{\mathrm{K}}$,

$$
\hat{S}=\int \mathrm{d} t N \mathcal{Q}\left(\frac{\pi_{\mathcal{A}}}{N}\right)-\left(S_{\text {ren }}+S_{\mathrm{K}}\right), \quad \pi_{\mathcal{A}} \equiv \frac{\delta S_{\text {ren }}}{\delta \mathcal{Q}},
$$

whose variation

$$
\delta \hat{S}=\int \mathrm{d} t N \mathcal{Q} \frac{\delta \pi_{\mathcal{A}}}{N}+\int \mathrm{d} t 4 N \beta \delta(\alpha-\kappa \beta)
$$

reveals the source $\pi_{\mathcal{A}} / N$ and expectation value

$$
\langle\mathcal{R}\rangle=N \mathcal{Q},
$$

To leading order in $N$, the RKKY interaction in eq. (5.44) thus amounts to adding to $\hat{S}$ the finite boundary term

$$
S_{\mathrm{RKKY}}=-N \int \mathrm{d} t \frac{1}{2} \lambda \mathcal{Q}^{2}, \quad \lambda \equiv N \lambda_{\mathrm{RKKY}}
$$

A variation of the generating functional in the deformed theory gives

$$
\delta\left(\hat{S}+S_{\mathrm{RKKY}}\right)=\int \mathrm{d} t N \mathcal{Q} \delta\left(\frac{\pi_{\mathcal{A}}}{N}-\lambda \mathcal{Q}\right)+\int \mathrm{d} t 4 N \beta \delta(\alpha-\kappa \beta)
$$

indicating that $\langle\mathcal{R}\rangle=N \mathcal{Q}$ remains unchanged, while the source for $\mathcal{R}$ changes as $\pi_{\mathcal{A}} / N \longrightarrow \pi_{\mathcal{A}} / N-\lambda \mathcal{Q}$. As a result, states that include the double-trace RKKY deformation but no single-trace deformation will be described by bulk solutions for $\mathcal{A}_{t}$ satisfying the boundary condition $\pi_{\mathcal{A}} / N-\lambda \mathcal{Q}=0$ with fixed $\lambda,{ }^{12}$ that is, from eq. (5.42),

$$
\lambda=-\frac{1}{\mathcal{Q}}\left[\mu+2 \mathcal{Q}^{+}\left(2 \alpha^{2}-2 \alpha \beta+\beta^{2}-\frac{1}{3} \frac{\beta^{3}}{\alpha}\right)\right] .
$$

We have thus determined the boundary terms in the action and the boundary conditions on $\phi$ and $\mathcal{A}_{t}$ that implement the double-trace Kondo and RKKY couplings in our holographic model. As for the third field in our ansatz, $a_{t}^{0}$, we fix its leading mode $Q^{0}$, which in the dual field theory fixes the representation of the impurities, as mentioned below eq. (5.21).

\footnotetext{
${ }^{12}$ We show in the appendix of ref. [15] that both Dirichlet and Neumann, and hence also mixed boundary conditions lead to normalisable solutions for a gauge field in $A d S_{2}$.
} 


\subsubsection{RG transformations}

We will now check that the holographic Kondo and RKKY couplings identified in the previous subsection behave correctly under RG transformations, and give a precise definition of the Kondo temperature $T_{\mathrm{K}}$. In holography, $\mathrm{RG}$ transformations can be implemented in the bulk by so-called Penrose-Brown-Henneaux (PBH) diffeomorphisms [285,286], which in our case reduce to translations of the radial coordinate (5.33),

$$
r \longrightarrow r+\ln (L)
$$

where $L$ is an arbitrary renormalisation length scale. ${ }^{13}$ This changes the induced asymptotic metric: $-e^{2 r} d t^{2} \rightarrow-e^{2 r} L^{-2} d t^{2}$. Sending $L \rightarrow 0$ thus amounts to 'zooming in' on the UV of the field theory, while sending $L \rightarrow \infty$ amounts to 'zooming out' to the IR.

The scalar $\phi(r)$ and $\mathcal{A}_{t}^{ \pm}$in radial gauge are both invariant under (5.54), implying

$$
\begin{aligned}
\alpha & \rightarrow L^{1 / 2} \alpha, \quad \beta \rightarrow L^{1 / 2}(\beta+\alpha \ln (L)), \\
\mathcal{Q}^{-} & \rightarrow L^{-1} \mathcal{Q}^{-}, \quad \mathcal{Q}^{+} \rightarrow L^{-1} \mathcal{Q}^{+}, \quad \mu^{-} \rightarrow \mu^{-} \\
\mu^{+} & \rightarrow \mu^{+}+\frac{2}{3} \mathcal{Q}^{+}\left[\left((\ln (L))^{2}-3 \ln (L)+6\right) \alpha^{2}-3(2-\ln (L)) \alpha \beta+3 \beta^{2}\right] \ln (L) .
\end{aligned}
$$

Using the identifications of our holographic Kondo and RKKY couplings from the previous subsection, $\kappa=\alpha / \beta$ and $\lambda$ in eq. (5.53), eqs. (5.55) give us the RG transformations

$$
\begin{gathered}
\kappa=\frac{\alpha}{\beta} \quad \longrightarrow \quad \kappa(L) \equiv \frac{\kappa}{1+\kappa \ln (L)}, \\
\lambda=\frac{-\mu-2 \mathcal{Q}^{+}\left(2 \alpha^{2}-2 \alpha \beta+\beta^{2}-\frac{1}{3} \frac{\beta^{3}}{\alpha}\right)}{\mathcal{Q}} \longrightarrow L \lambda .
\end{gathered}
$$

Note that the inhomogeneous RG transformations of $\mu=\mu^{+}-\mu^{-}$and $\beta$ conspire in precisely the right way to produce a homogeneous RG transformation for $\lambda$. Eq. (5.56a) confirms that $\kappa$ is marginally relevant or irrelevant if in the UV $\kappa<0$ (AFM Kondo coupling) or $\kappa>0$ (FM Kondo coupling), respectively. ${ }^{14}$ Eq. (5.56b) confirms that $\lambda$ is a relevant coupling of dimension one. The beta function for $\kappa, \beta_{\kappa}=-L \partial_{L} \kappa(L)=\kappa^{2}(L)$,

\footnotetext{
${ }^{13}$ The argument of the logarithm in eq. (5.54) is made dimensionless by the unit $A d S_{3}$ radius.

${ }^{14}$ In contrast, if we had started with a Neumann boundary condition for $\phi$ instead of Dirichlet, then $\kappa$ 's RG transformation would be $\kappa \longrightarrow(1+\kappa \ln (L)) / \kappa$, which is always marginally relevant since $\kappa$ grows in the IR, $L \rightarrow \infty$, for both $\kappa<0$ and $\kappa>0$ in the UV.
} 
shows that an AFM Kondo coupling is asymptotically free and diverges in the IR at a dynamically generated scale: Fixing the value of $\kappa$ at a fixed but arbitrary length scale $L^{\prime}$, we find from eq. (5.56a) that

$$
\kappa(L)=\frac{\kappa\left(L^{\prime}\right)}{1+\kappa\left(L^{\prime}\right) \ln \left(L / L^{\prime}\right)}
$$

diverges at the length scale $L^{\prime} e^{-1 / \kappa\left(L^{\prime}\right)}$, which is a physical quantity, being invariant under re-scalings of $L^{\prime}$. We then define the Kondo temperature as

$$
T_{\mathrm{K}} \equiv \frac{1}{2 \pi} \frac{1}{L^{\prime}} e^{1 / \kappa\left(L^{\prime}\right)},
$$

where the factor of $1 /(2 \pi)$ will be convenient in section 5.5 .

\subsection{The two-impurity phase diagram}

In this section we determine the phase diagram of our two-impurity model in the plane of $\lambda /\left(2 \pi T_{\mathrm{K}}\right)$ versus $T / T_{\mathrm{K}}$, with our holographic RKKY coupling $\lambda$ defined in eq. (5.51) and the Kondo temperature $T_{\mathrm{K}}$ defined in eq. (5.58). To do so we will solve the equations of motion (5.32), subject to the boundary conditions discussed in subsection 5.4.2.

We know one exact solution which exists for all values of $Q^{0}, \lambda /\left(2 \pi T_{\mathrm{K}}\right)$, and $T / T_{\mathrm{K}}$, namely the trivial solution $a_{t}^{0}=\mathcal{A}_{t}^{+}+\mathcal{A}_{t}^{-}=Q^{0} / z+\mu^{0}$ with $\phi(z)=0$ and $\mathcal{A}_{t}(z)=$ $\mathcal{A}_{t}^{+}(z)-\mathcal{A}_{t}^{-}(z)=0$. It is dual to a trivial state with $\left\langle\left|\mathcal{O}_{\mathrm{I}}\right|\right\rangle=\left\langle\left|\mathcal{O}_{\mathrm{II}}\right|\right\rangle=0$ and $\left\langle R^{1}\right\rangle=$ $\left\langle R^{2}\right\rangle=0$, where via eq. (5.10) the latter implies $\left\langle S_{\mathrm{I}}^{A} S_{\mathrm{II}}^{A}\right\rangle=0$, so the two impurity spins are neither Kondo screened nor correlated with one another. To describe non-trivial states with non-zero $\left\langle\left|\mathcal{O}_{\mathrm{I}}\right|\right\rangle=\left\langle\left|\mathcal{O}_{\text {II }}\right|\right\rangle$ and/or non-zero $\left\langle R^{1}\right\rangle=\left\langle R^{2}\right\rangle$, we must construct non-trivial solutions for $a_{t}^{0}, \mathcal{A}_{t}$, and $\phi$. Moreover, to determine whether a non-trivial state has lower free energy than the trivial state we must determine whether the nontrivial solutions have smaller on-shell Euclidean action than the trivial solution.

The equations of motion are second order, so we need two boundary conditions for each field, $a_{t}^{0}, \mathcal{A}_{t}$, and $\phi$. As summarised at the end of subsection 5.4.2, fixing the impurities' representation and the Kondo and RKKY couplings in the UV gives us three conditions at the $A d S_{2}$ boundary. We impose the remaining three conditions at the horizon $z=z_{H}$. First, we demand regularity of the Abelian one-form $a_{t}^{0}$ at the 
horizon, which requires $a_{t}^{0}\left(z_{H}\right)=0$. Second, we require regularity of $\phi$ at the horizon. In a near-horizon expansion of $\phi$, the leading modes are $\ln \left(z-z_{H}\right)$ and a constant. Regularity requires the $\ln \left(z-z_{H}\right)$ term to vanish, which in turn implies $\mathcal{A}_{t}^{+}\left(z_{H}\right)=0$. That implies, via the definitions in eq. (5.30), $\mathcal{A}_{t}^{-}\left(z_{H}\right)=0$ and hence $\mathcal{A}_{t}\left(z_{H}\right)=0$.

We have only been able to construct non-trivial solutions numerically. However, in subsections 5.5.1 and 5.5.2 we will constrain their properties as much as we can without numerics. We then resort to numerics in subsection 5.5.3, where we discuss our main numerical results, including the phase diagram of our model.

\subsubsection{Properties of non-trivial solutions}

In the trivial solution, $\phi=0$ and hence $J_{t}^{0}=0$. In that case, $a_{t}^{0}$ 's equation of motion, eq. (5.19) with $b=0$, requires the flux of $a_{t}^{0}$ to be constant in $z$. In other words, in the absence of charged sources Gauss's law requires the flux to be a constant, fixed by the flux $Q^{0}$ at the $A d S_{2}$ boundary. Translating to the dual field theory, the impurities are unscreened in the trivial state. By contrast, a non-trivial solution has $\phi \neq 0$ and $J_{t}^{0} \neq 0$, so that $\phi$ removes flux from $a_{t}^{0}$ as $z$ increases, representing the screening of the impurities in the IR in a non-trivial state.

For our model we can prove that $\phi=0$ if and only if $a_{t}^{1}=a_{t}^{2}=0$, and conversely that $\phi \neq 0$ if and only if $a_{t}^{1}=a_{t}^{2} \neq 0$, as follows. First suppose $\phi=0$. In that case, the equations of motion of all the gauge fields can be solved exactly, in particular $\mathcal{A}_{t}=\mathcal{Q} / z+\mu$. The condition $\mathcal{A}_{t}\left(z_{H}\right)=0$ implies $\mu=-\mathcal{Q} / z_{H}$. However, when $\phi=0$ the boundary condition (5.53) for the RKKY coupling reduces to $\mu=-\lambda \mathcal{Q}$. Clearly, in the generic case $\lambda \neq 1 / z_{H}$, the only consistent solution has $\mu=\mathcal{Q}=0$ and hence $\mathcal{A}_{t}=0$. Eqs. (5.26) and (5.27) then imply $a_{t}^{1}=a_{t}^{2}=0$. Conversely, suppose $\phi \neq 0$. In that case, the solution for $\mathcal{A}_{t}^{+}$is not $\mathcal{Q}^{+} / z+\mu^{+}$and so $\mu^{+} \neq-\mathcal{Q}^{+} / z_{H}$. Using $\mathcal{A}_{t}^{-}=$ $\mathcal{Q}^{-} / z+\mu^{-}$with $\mu^{-}=-\mathcal{Q}^{-} / z_{H}$ and $\mathcal{A}_{t}^{-}=\mathcal{A}_{t}^{+}-\mathcal{A}_{t}$, we find $\mu^{+}-\mu=-\left(\mathcal{Q}^{+}-\mathcal{Q}\right) / z_{H}$. As a result, $\mu^{+} \neq-\mathcal{Q}^{+} / z_{H}$ implies $\mu \neq-\mathcal{Q} / z_{H}$. That condition forbids $\mathcal{A}_{t}=0$, which has $\mu=\mathcal{Q}=0$ and hence trivially has $\mu=-\mathcal{Q} / z_{H}$. We thus learn that $\phi \neq 0$ implies $\mathcal{A}_{t} \neq 0$. This completes our proof. Translating to the field theory, we have learned that, for our model and within our ansatz, if either of $\mathcal{O}_{\text {I }}=\mathcal{O}_{\text {II }}$ or $R^{1}=R^{2}$ has vanishing 
expectation value then so does the other, while if either acquires a non-zero expectation value then so must the other. In other words, the absence of Kondo screening is always accompanied by the absence of correlations between the two impurity spins and vice versa, while Kondo screening is always accompanied by non-zero correlations between the two impurity spins and vice versa.

Our model thus admits two possible phases, distinguished by their symmetries. The RKKY interaction explicitly breaks the auxiliary $U(2)$ down to $U(1)^{0} \times U(1)^{3}$, where $U(1)^{0}$ and $U(1)^{3}$ are generated by $\tau^{0}$ and $\tau^{3}$, respectively. The trivial solution preserves $U(1)^{0} \times U(1)^{3}$ and the Chern-Simons $U(1)$, dual to the electromagnetic $U(1)$, while a non-trivial solution breaks $U(1)^{0} \times U(1)^{3} \times U(1)$ to a subgroup. Specifically, both $\phi \neq 0$ and $\mathcal{A}_{t} \neq 0$ break $U(1)^{3}$ completely, and $\phi \neq 0$ breaks $U(1)^{0} \times U(1)$ to the diagonal. In field theory terms, the two possible phases in our system are the trivial phase, where $\left\langle\left|\mathcal{O}_{\mathrm{I}}\right|\right\rangle=\left\langle\left|\mathcal{O}_{\mathrm{II}}\right|\right\rangle=0$ and $\left\langle R^{1}\right\rangle=\left\langle R^{2}\right\rangle=0$ and so $U(1)^{0} \times U(1)^{3} \times U(1)$ is preserved, and the non-trivial phase, where $\left\langle\left|\mathcal{O}_{\mathrm{I}}\right|\right\rangle=\left\langle\left|\mathcal{O}_{\mathrm{II}}\right|\right\rangle \neq 0$ and $\left\langle R^{1}\right\rangle=\left\langle R^{2}\right\rangle \neq 0$ spontaneously break $U(1)^{3}$ completely and break $U(1)^{0} \times U(1)$ to the diagonal. ${ }^{15}$

We can constrain the possible phases in our model even further, as follows. In the probe limit, we compute only the leading $\mathcal{O}(N)$ contributions to $\left\langle\left|\mathcal{O}_{\mathrm{I}}\right|\right\rangle=\left\langle\left|\mathcal{O}_{\mathrm{II}}\right|\right\rangle$ and $\left\langle R^{1}\right\rangle=\left\langle R^{2}\right\rangle$. In this limit the spin-spin correlator is thus, via eqs. (5.10) and (5.50),

$$
\left\langle S_{\mathrm{I}}^{A} S_{\mathrm{II}}^{A}\right\rangle=-\frac{1}{2} N^{2} \mathcal{Q}^{2}+\mathcal{O}(N)
$$

where $\mathcal{Q}$ is $\mathcal{O}\left(N^{0}\right)$. Eq. (5.59) shows that we will have access only to the order $N^{2}$ contribution of AFM spin-spin correlations, $\left\langle S_{\mathrm{I}}^{A} S_{\mathrm{II}}^{A}\right\rangle<0$.

\subsubsection{Group-theory results}

In fact we can show, using group theory alone, that at large $N$ the leading contribution to $\left\langle S_{\mathrm{I}}^{A} S_{\mathrm{II}}^{A}\right\rangle$ in FM eigenstates is always order $N$ and in AFM eigenstates is order $N^{2}$,

\footnotetext{
${ }^{15}$ These two phases are also distinguished by their phase shifts. As in the holographic singleimpurity Kondo model of ref. [18], in our model the phase shift that accompanies Kondo screening appears holographically as a Wilson line of the Chern-Simons field in the $x$ direction: if we compactify the $x$ direction then the phase shift is $\propto \oint_{x} A$. Eq. (5.17b) shows that if $\mathcal{A}_{t}^{+} \neq 0, \phi \neq 0$ then $A_{x} \neq 0$, while if $\mathcal{A}_{t}^{+}=\phi=0$ then $A_{x}=0$. Translating to the field theory, we find that a phase shift occurs in non-trivial states but not in the trivial state. In fact, the Chern-Simons field's only role is to implement the phase shift and it will play no further role in the remainder of this section.
} 
and that the vast majority of eigenstates are AFM. To our knowledge, the following results have never before appeared in the literature about the Kondo effect.

The tensor product of two identical anti-symmetric representations $\rho_{\mathrm{UV}}$, each with a Young tableau consisting of a single column with $q$ boxes, is

$$
\rho_{\mathrm{UV}} \otimes \rho_{\mathrm{UV}}=\sum_{p=0}^{p_{\max }} \rho_{p}
$$

where the irreducible representation $\rho_{p}$ has a Young tableau with two columns, the first with $(q+p)$ boxes and the second with $(q-p)$ boxes, and where

$$
p_{\max }= \begin{cases}q, & q \leq N / 2 \\ N-q, & q>N / 2 .\end{cases}
$$

For a given representation $\rho_{p}$, we can express $\left\langle S_{\mathrm{I}}^{A} S_{\mathrm{II}}^{A}\right\rangle$ in terms of the quadratic Casimir of that representation, $C\left(\rho_{p}\right)$ :

$$
\begin{aligned}
\left.\left\langle S_{\mathrm{I}}^{A} S_{\mathrm{II}}^{A}\right\rangle\right|_{\rho_{p}} & =\left.\frac{1}{2}\left(\left(S_{\mathrm{I}}+S_{\mathrm{II}}\right)^{A}\left(S_{\mathrm{I}}+S_{\mathrm{II}}\right)^{A}-S_{\mathrm{I}}^{A} S_{\mathrm{I}}^{A}-S_{\mathrm{II}}^{A} S_{\mathrm{II}}^{A}\right)\right|_{\rho_{p}} \\
& =\frac{1}{2} C\left(\rho_{p}\right)-\left.\frac{1}{2}\left(S_{\mathrm{I}}^{A} S_{\mathrm{I}}^{A}+S_{\mathrm{II}}^{A} S_{\mathrm{II}}^{A}\right)\right|_{\rho_{p}} .
\end{aligned}
$$

Using [287]

$$
C\left(\rho_{p}\right)=N(N+2) \frac{q}{N}\left(1-\frac{q}{N}\right)-p(p+1),
$$

as well as, for any of the $\rho_{p}$,

$$
\left\langle S_{\mathrm{I}}^{A} S_{\mathrm{I}}^{A}\right\rangle=\frac{1}{2}(N+1) \chi_{\mathrm{I} \alpha}^{\dagger} \chi_{\mathrm{I} \alpha}\left(1-\frac{\chi_{\mathrm{I} \alpha}^{\dagger} \chi_{\mathrm{I} \alpha}}{N}\right)=\frac{1}{2} N(N+1) \frac{q}{N}\left(1-\frac{q}{N}\right),
$$

we find for our identical impurity spins, $S_{\mathrm{I}}^{A} S_{\mathrm{I}}^{A}=S_{\mathrm{II}}^{A} S_{\mathrm{II}}^{A}$ :

$$
\left.\left\langle S_{\mathrm{I}}^{A} S_{\mathrm{II}}^{A}\right\rangle\right|_{\rho_{p}}=\frac{1}{2} N \frac{q}{N}\left(1-\frac{q}{N}\right)-\frac{1}{2} p(p+1) .
$$

Clearly, $\left.\left\langle S_{\mathrm{I}}^{A} S_{\mathrm{II}}^{A}\right\rangle\right|_{\rho_{p}}$ decreases monotonically as $p$ increases. As a result, the FM ground state, which maximizes $\left\langle S_{\mathrm{I}}^{A} S_{\mathrm{II}}^{A}\right\rangle$, has $p=0$, while the AFM ground state, which minimises $\left\langle S_{\mathrm{I}}^{A} S_{\mathrm{II}}^{A}\right\rangle$, has $p=p_{\max }$. In fact, in the large- $N$ limit with $q / N$ of order one, eq. (5.65) shows that for any FM representation the leading contribution to $\left\langle S_{\mathrm{I}}^{A} S_{\mathrm{II}}^{A}\right\rangle$ is order $N$. Moreover, $\left\langle S_{\mathrm{I}}^{A} S_{\mathrm{II}}^{A}\right\rangle>0$ only for $p$ up to a critical value,

$$
p_{\text {crit }}=\frac{1}{2}\left(\sqrt{1+4 N \frac{q}{N}\left(1-\frac{q}{N}\right)}-1\right),
$$


which scales as $\sqrt{N}$ when $N \rightarrow \infty$ with $q / N$ of order one. The total number of representations in eq. (5.60) scales as $N$ as $N \rightarrow \infty\left(p_{\max }\right.$ scales as $\left.N\right)$, so only a small fraction of representations of order $\sqrt{N} / N=1 / \sqrt{N}$ are FM. For an AFM ground state, using $p_{\max }$ from eq. (5.61), we find

$$
\left.\left\langle S_{\mathrm{I}}^{A} S_{\mathrm{II}}^{A}\right\rangle\right|_{\rho_{p_{\max }}}= \begin{cases}-\frac{1}{2} N(N+1)\left(\frac{q}{N}\right)^{2}, & q \leq N / 2, \\ -\frac{1}{2} N(N+1)\left(1-\frac{q}{N}\right)^{2}, & q>N / 2\end{cases}
$$

which clearly scales as $N^{2}$ as $N \rightarrow \infty$ with $q / N$ of order one. Moreover, inserting $p=P p_{\max }$ with $0 \leq P \leq 1$ into eq. (5.65) reveals $\left\langle S_{\mathrm{I}}^{A} S_{\mathrm{II}}^{A}\right\rangle$ with $\left\langle S_{\mathrm{I}}^{A} S_{\mathrm{II}}^{A}\right\rangle<0$ scales linearly in $N$ only for the small fraction of eigenstates for which $P$ is order $1 / \sqrt{N}$. In other words, for the vast majority of AFM eigenstates $\left\langle S_{\mathrm{I}}^{A} S_{\mathrm{II}}^{A}\right\rangle$ scales as $N^{2}$.

We have thus shown that, in the large- $N$ limit with $q / N$ order one, the leading contribution to $\left\langle S_{\mathrm{I}}^{A} S_{\mathrm{II}}^{A}\right\rangle$ is order $N$ in FM eigenstates and order $N^{2}$ in the vast majority of AFM eigenstates, and that the latter vastly outnumber the former.

In our holographic model, according to eq. (5.59) we will only be able to distinguish between superpositions of AFM eigenstates with $\left\langle S_{\mathrm{I}}^{A} S_{\mathrm{II}}^{A}\right\rangle \neq 0$ of order $N^{2}$ and uncorrelated spins, $\left\langle S_{\mathrm{I}}^{A} S_{\mathrm{II}}^{A}\right\rangle=0$. We thus expect to find non-trivial solutions only for AFM RKKY coupling $\lambda / T_{\mathrm{K}}>0$ and only the trivial solution for FM RKKY coupling $\lambda / T_{\mathrm{K}}<0 .{ }^{16}$ Therefore, only one quantum phase transition is possible in our model: a transition from the trivial phase with uncorrelated spins and no Kondo screening to the non-trivial phase with AFM spin-spin correlations of order $N^{2}$ and Kondo screening. Although our numerical results will not extend down to exactly $T=0$, we will find highly suggestive evidence for such a quantum phase transition in our model.

In contrast, as reviewed in subsection 5.2.2 the original large- $N$ two-impurity Kondo model of refs. [224,225] exhibits a quantum phase transition from a FM ground state with Kondo screening to an AFM ground state without Kondo screening. The difference in the phase diagram can be traced back to two crucial differences in the model. First,

\footnotetext{
${ }^{16}$ The appearance of non-trivial solutions for only one sign of a double-trace coupling is in fact generic in large- $N$ field theory and in holography [72,283,284]. Adding a double-trace coupling shifts the quantum effective potential and generically will change the ground state only for one sign of the double-trace coupling constant, much the way a mass term added to a scalar field theory with quartic interaction will trigger scalar condensation only for negative mass-squared.
} 
refs. [224,225] employ a vector-like large- $N$ limit, which allows access to $\mathcal{O}(N)$ FM spinspin correlations. We instead employ a matrix-like large- $N$ limit, which only allows access to the $\mathcal{O}\left(N^{2}\right)$ AFM spin-spin correlations. Second, refs. [224, 225] considered the very special case of two impurities in totally anti-symmetric representations with exactly $q=N / 2$ boxes. In that special case, the two impurities lock into a singlet in the AFM limit and no Kondo screening occurs. Our bottom-up model, however, is too crude to allow fine-tuning to that special case: generically, even at strong AFM RKKY coupling our ground state will not be a singlet and Kondo screening will occur.

\subsubsection{Numerical results}

We now turn to the numerical solution of the equations of motion (5.32) and to the numerical evaluation of the Euclidean on-shell action. We first re-scale all quantities by appropriate powers of $z_{H}=1 /(2 \pi T)$ to obtain dimensionless coordinates and fields,

$$
\left(z / z_{H}, t / z_{H}\right) \rightarrow(z, t), \quad z_{H} \mathcal{A}_{t}^{ \pm} \rightarrow \mathcal{A}_{t}^{ \pm}, \quad \phi \rightarrow \phi .
$$

After these re-scalings, the $A d S_{3}$ boundary is at $z=0$ while the horizon is at $z=1$. The dimensionless $\phi$ now has the asymptotic expansion

$$
\phi=\sqrt{z}\left(\alpha_{T} \ln (z)+\beta_{T}\right)+\ldots
$$

where $\alpha_{T}$ and $\beta_{T}$ are related to the original $\alpha$ and $\beta$ in eq. (5.35) as

$$
\alpha_{T} \equiv \sqrt{z_{H}} \alpha, \quad \beta_{T} \equiv \sqrt{z_{H}}\left(\beta+\alpha \ln \left(z_{H}\right)\right)
$$

We then define $\kappa_{T} \equiv \alpha_{T} / \beta_{T}$, which is related to $\kappa=\alpha / \beta$ as

$$
\kappa_{T} \equiv \frac{\alpha_{T}}{\beta_{T}}=\frac{\kappa}{1+\kappa \ln \left(z_{H}\right)}
$$

Comparing eq. (5.71) to eq. (5.56a) reveals that $\kappa_{T}$ is $\kappa(L)$ evaluated at the length scale $L=z_{H}=1 /(2 \pi T)$. Using our definition (5.58) of $T_{\mathrm{K}}$, we can write $\kappa_{T}$ as

$$
\kappa_{T}=\frac{1}{\ln \left(T_{\mathrm{K}} / T\right)},
$$


whose simple form justifies the choice of the $1 /(2 \pi)$ factor in eq. (5.58). The dimensionless $\mathcal{A}_{t}^{+}$has an asymptotic expansion

$$
\begin{gathered}
\mathcal{A}_{t}^{+}=\frac{\mathcal{Q}^{+}}{z}+\mathcal{Q}^{+}\left[c_{T}^{(3)}(\ln (z))^{3}+c_{T}^{(2)}(\ln (z))^{2}+c_{T}^{(1)} \ln (z)\right]+\mu_{T}^{+}+\ldots, \\
\mu_{T}^{+} \equiv z_{H} \mu^{+}+\mathcal{Q}^{+}\left[c_{T}^{(3)}\left(\ln \left(z_{H}\right)\right)^{3}-c_{T}^{(2)}\left(\ln \left(z_{H}\right)\right)^{2}+c_{T}^{(1)} \ln \left(z_{H}\right)\right], \\
c_{T}^{(3)} \equiv \frac{2}{3} \alpha_{T}^{2}, \quad c_{T}^{(2)} \equiv-2 \alpha_{T}^{2}+2 \alpha_{T} \beta_{T}, \quad c_{T}^{(1)} \equiv 4 \alpha_{T}^{2}-4 \alpha_{T} \beta_{T}+2 \beta_{T}^{2} .
\end{gathered}
$$

After the re-scalings in eq. (5.68), the boundary condition $\lambda=\pi_{\mathcal{A}} /(N \mathcal{Q})$ becomes

$$
\begin{gathered}
\frac{\lambda}{2 \pi T}=\frac{1}{2 \pi T} \frac{\pi_{\mathcal{A}}}{N \mathcal{Q}}=-\frac{1}{\mathcal{Q}}\left[\mu_{T}+2 \mathcal{Q}^{+}\left(2 \alpha_{T}^{2}-2 \alpha_{T} \beta_{T}+\beta_{T}^{2}-\frac{1}{3} \frac{\beta_{T}^{3}}{\alpha_{T}}\right)\right] \\
\mu_{T} \equiv \mu_{T}^{+}-z_{H} \mu^{-}
\end{gathered}
$$

As mentioned below eq. (5.32), $\mathcal{A}_{t}^{-}$decouples from $\mathcal{A}_{t}^{+}$and $\phi$, and can be solved for exactly: the solution obeying the boundary condition $\mathcal{A}_{t}^{-}(z=1)=0$ is

$$
\mathcal{A}_{t}^{-}=\frac{\mathcal{Q}^{-}}{z}+\mu^{-}, \quad \mu^{-}=-\frac{\mathcal{Q}^{-}}{z_{H}} .
$$

We have been able to obtain only numerical solutions for $\mathcal{A}_{t}^{+}$and $\phi$, using the following numerical shooting procedure. First, we fix $Q^{0}$, and then choose a target value for $\mathcal{Q}^{+}$. We then know $\mathcal{Q}^{-}=Q^{0}-\mathcal{Q}^{+}$and $\mathcal{Q}=2 \mathcal{Q}^{+}-Q^{0}$ from eq. (5.31). Choosing $\mathcal{Q}^{+}$also fixes $M^{2}=-1 / 4+\left(\mathcal{Q}^{+}\right)^{2}$, so that $\phi$ 's equation of motion is fixed. We then demand that the $\ln (z-1)$ term in $\phi$ 's near-horizon expansion vanish and that $\mathcal{A}_{t}^{+}(z=1)=0$. Two free parameters then remain at the horizon, $\phi(z=1) \equiv \phi_{H}$ and $\partial_{z} \mathcal{A}_{t}^{+}(z=1)$. We fix $\phi_{H}$ and dial through $\partial_{z} \mathcal{A}_{t}^{+}(z=1)$ values, for each value obtaining numerical solutions for $\mathcal{A}_{t}^{+}$and $\phi$, but retaining only those solutions with the target value of $\mathcal{Q}^{+}$. We then extract $\alpha_{T}$ and $\beta_{T}$ from the asymptotics of $\phi$ 's numerical solution, which gives us $\kappa_{T}=\alpha_{T} / \beta_{T}$ and, via eq. (5.70), $\left\langle\left|\mathcal{O}_{\mathrm{I}}\right|\right\rangle=\left\langle\left|\mathcal{O}_{\text {II }}\right|\right\rangle=-4 N \beta$. We also extract $\mu_{T}$ from the solution for $\mathcal{A}_{t}=\mathcal{A}_{t}^{+}-\mathcal{A}_{t}^{-}$using eqs. (5.73)-(5.75), which gives us $\lambda /(2 \pi T)$ via eq. (5.74a). Using $T / T_{\mathrm{K}}=e^{-1 / \kappa_{T}}$ from eq. (5.72) we then also find $\frac{T}{T_{\mathrm{K}}} \frac{\lambda}{2 \pi T}=\frac{\lambda}{2 \pi T_{\mathrm{K}}}$. We thus obtain all the one-point functions for a given point $\left(\lambda /\left(2 \pi T_{\mathrm{K}}\right), T / T_{\mathrm{K}}\right)$ in the phase diagram. We then change $\phi_{H}$ and repeat the process of dialing through $\partial_{z} \mathcal{A}_{t}^{+}(z=1)$ values to obtain the same target value of $\mathcal{Q}^{+}$, but 
now obtaining new values of $T / T_{\mathrm{K}}$ and $\lambda /\left(2 \pi T_{\mathrm{K}}\right)$. In this way, we generate the phase diagram in the $\left(\lambda /\left(2 \pi T_{\mathrm{K}}\right), T / T_{\mathrm{K}}\right)$ plane by moving along curves of constant $\mathcal{Q}^{+}$, or equivalently of constant $\mathcal{Q}=2 \mathcal{Q}^{+}-Q^{0}$, which via eqs. (5.50) and (5.59) means curves of constant $\left\langle R^{1}\right\rangle=\left\langle R^{2}\right\rangle$ and $\left\langle S_{\mathrm{I}}^{A} S_{\mathrm{II}}^{A}\right\rangle$.

We also numerically compute the renormalised free energy $\mathcal{F}$ of each non-trivial solution. If we Wick-rotate to Euclidean signature and then compactify our dimensionless Euclidean time direction into a circle of circumference $2 \pi$, then $\mathcal{F}$ is $T$ times the renormalised Euclidean on-shell action. Because all of our solutions are static, $\mathcal{F}$ is simply $2 \pi T$ times an integral over $z$ in the Euclidean on-shell action, which we performed numerically, plus the boundary terms described in subsection 5.4.2. The Chern-Simons gauge field's contribution to $\mathcal{F}$ vanishes.

The trivial solution, $a_{t}^{0}=Q^{0}\left(\frac{1}{z}-1\right)$ with all other fields vanishing, exists everywhere in the $\left(\lambda /\left(2 \pi T_{\mathrm{K}}\right), T / T_{\mathrm{K}}\right)$ plane and has $\mathcal{F}=-\left(Q^{0}\right)^{2} / 2$. If we define $\Delta \mathcal{F}$ as $-\left(Q^{0}\right)^{2} / 2$ minus the value of $\mathcal{F}$ for a non-trivial solution, then $\Delta \mathcal{F}>0$ means the non-trivial solution is thermodynamically preferred over the trivial solution, and vice-versa for $\Delta \mathcal{F}<0$. However, our numerical results were not always sufficiently accurate to determine the sign of $\Delta \mathcal{F}$. After a large number of iterations of our numerical shooting, the change in our results for $\alpha_{T}, \beta_{T}$, and $\mu_{T}$ between iterations stabilized to roughly $10^{-7}$. Assuming convergence to the actual values, we thus took $10^{-7}$ as the uncertainty in our numerical results for $\alpha_{T}, \beta_{T}$, and $\mu_{T}$. Numerically, we found that obtaining $\lambda /\left(2 \pi T_{\mathrm{K}}\right)$ of order one required $\mathcal{Q}^{+} \geq 10^{-3}$. These two bounds together imply, via eq. (5.74a), an uncertainty in our results for $\lambda /\left(2 \pi T_{\mathrm{K}}\right)$ of roughly $10^{-4}$. The on-shell action includes a boundary term $\propto \lambda /\left(2 \pi T_{\mathrm{K}}\right)$, eq. (5.51), so our numerical results for $\Delta \mathcal{F}$ were also accurate up to a threshold of only $10^{-4}$. In some cases, our numerical result for $|\Delta \mathcal{F}|$ was less than $10^{-4}$, so that we could not determine the sign of $\Delta \mathcal{F}$ and hence not conclude if the non-trivial solution was preferred over the trivial solution.

The equations of motion (5.32) and the bulk integral over $z$ in $\Delta \mathcal{F}$ are invariant under independent sign flips of $\mathcal{A}_{t}^{-}, \mathcal{A}_{t}^{+}$, and $\phi$. The boundary terms in $\Delta \mathcal{F}$, from the Kondo and RKKY double-trace deformations, are invariant under two of these $\mathbb{Z}_{2}$ symmetries. First, taking $\phi \rightarrow-\phi$ with $\mathcal{A}_{t}^{-}, \mathcal{A}_{t}^{+}$unchanged will take $\phi_{H} \rightarrow-\phi_{H}$, 
$\alpha_{T} \rightarrow-\alpha_{T}, \beta_{T} \rightarrow-\beta_{T}$. However, $\kappa_{T} \equiv \alpha_{T} / \beta_{T}$ and $\lambda /(2 \pi T)$ in eq. (5.74a) will be invariant, and hence the boundary terms in $\Delta \mathcal{F}$ will be invariant. Without loss of generality we thus restricted to $\phi_{H}>0$. Second, taking $\mathcal{A}_{t}^{-} \rightarrow-\mathcal{A}_{t}^{-}, \mathcal{A}_{t}^{+} \rightarrow-\mathcal{A}_{t}^{+}$ with $\phi$ unchanged sends $\mu_{T} \rightarrow-\mu_{T}, Q^{0} \rightarrow-Q^{0}, \mathcal{Q} \rightarrow-\mathcal{Q}$ with $\alpha_{T}, \beta_{T}$ unchanged. Again, $\kappa_{T}$ and $\lambda /(2 \pi T)$ will be unchanged, and hence the boundary terms in $\Delta \mathcal{F}$ will be unchanged. Without loss of generality we thus restricted to $Q^{0}<0$.

Our main result is fig. 5.1, the phase diagrams of our model in the $\left(\lambda /\left(2 \pi T_{\mathrm{K}}\right), T / T_{\mathrm{K}}\right)$ plane, for $Q^{0}=-1,-1.2$, and -1.4 . In fig. 5.1, each black dot represents a non-trivial numerical solution. As anticipated in subsection 5.5.1, every non-trivial solution had both $\phi \neq 0$ and $a_{t}^{1}=a_{t}^{2} \neq 0$. As anticipated in subsection 5.5.2 we found non-trivial solutions only for AFM RKKY coupling $\lambda /\left(2 \pi T_{\mathrm{K}}\right)>0$. In fact, we found non-trivial solutions only inside the regions bounded by the dotted lines in each of figs 5.1 (a.), (b.), (c.), and (d.). In each case, the dotted diagonal line is $T / T_{\mathrm{K}}=\lambda /\left(2 \pi T_{\mathrm{K}}\right)$, while the horizontal dotted line was determined by a linearised stability analysis, as follows.

As mentioned before, in our model a fluctuation of $\phi$ about the trivial solution obeys the same equation of motion and boundary conditions as in the holographic single-impurity model of ref. [18], but with $\mathcal{A}_{t}^{+}$replacing $a_{t}^{0}$. A central result of ref. [18] was that the fluctuation of $\phi$ became unstable below a critical $T$ that depended on $Q^{0}$. Moreover, that critical $T$ decreased as $\left|Q^{0}\right|$ increased, intuitively because an impurity spin in a larger-dimensional representation is more difficult to screen. The linearised analysis thus guaranteed that a phase transition must occur. The same result applies in our model, but with $\mathcal{Q}^{+}$replacing $Q^{0}$. However, because fluctuations of $\phi$ and $\mathcal{A}_{t}^{+}$decouple at the linearised order, the instability of $\phi$ is independent of $\lambda /\left(2 \pi T_{\mathrm{K}}\right)$, so it tells us nothing about the values of $\lambda /\left(2 \pi T_{\mathrm{K}}\right)$ where the trivial phase will become unstable. Given $\mathcal{Q}^{+}=\frac{1}{2}\left(Q^{0}+\mathcal{Q}\right)$, the minimal value of $\left|\mathcal{Q}^{+}\right|$is $\left|Q^{0} / 2\right|$, which determines the maximal critical $T / T_{\mathrm{K}}$ above which the trivial phase must be stable. In each of fig. 5.1 the horizontal dotted lines denote that maximal critical $T / T_{\mathrm{K}}$. As we increase $\left|Q^{0}\right|$, going from fig. 5.1 (b.) to fig. 5.1 (d.), the horizontal dotted line moves to smaller $T / T_{\mathrm{K}}$, as expected. Fig. 5.1 shows that for sufficiently large $\lambda /\left(2 \pi T_{\mathrm{K}}\right)$, non-trivial solutions appear already at the maximal critical $T / T_{\mathrm{K}}$. In contrast, for smaller $\lambda /\left(2 \pi T_{\mathrm{K}}\right)$, and 


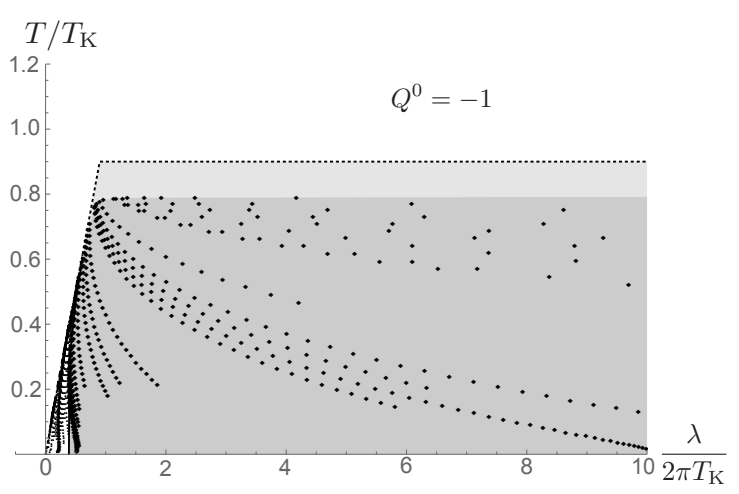

(a.)

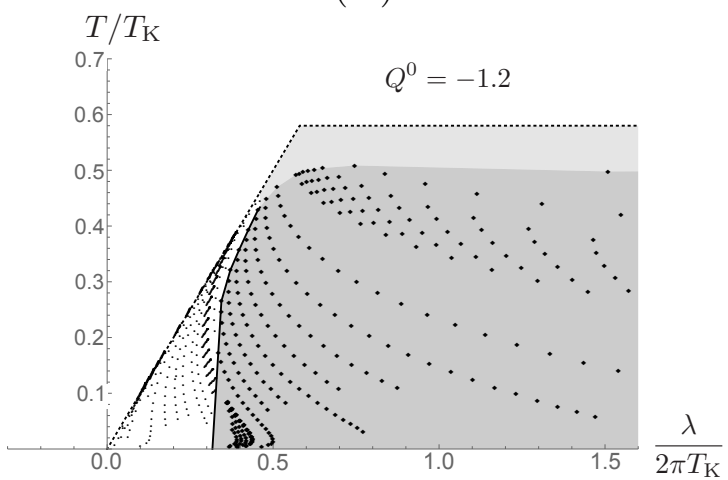

(c.)

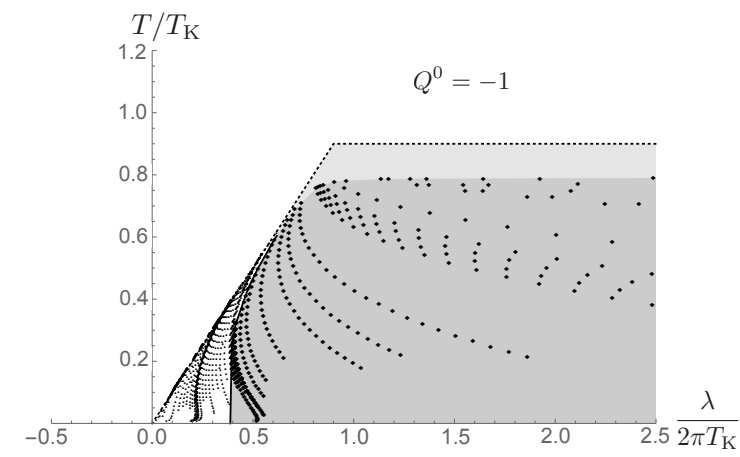

(b.)

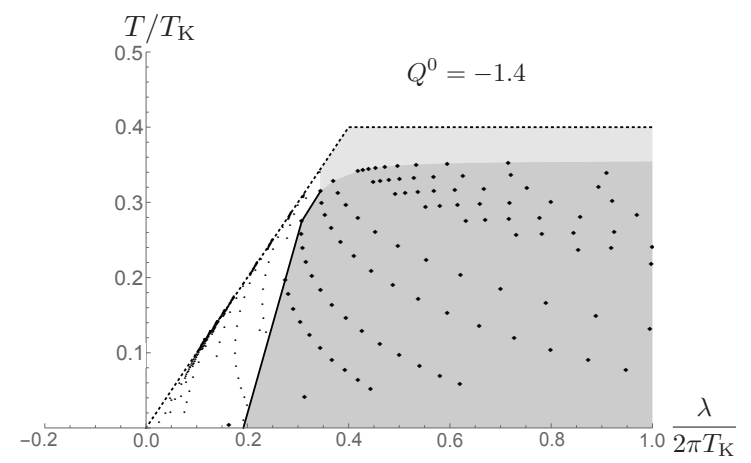

(d.)

Figure 5.1: Phase diagrams of our model for (a.) $Q^{0}=-1$ with $\lambda /\left(2 \pi T_{\mathrm{K}}\right) \in(-0.5,10)$, (b.) $Q^{0}=-1$ again but zooming in on $\lambda /\left(2 \pi T_{\mathrm{K}}\right) \in(-0.5,2.5)$, (c.) $Q^{0}=-1.2$ with $\lambda /\left(2 \pi T_{\mathrm{K}}\right) \in(-0.3,1.6)$, and (d.) $Q^{0}=-1.4$ with $\lambda /\left(2 \pi T_{\mathrm{K}}\right) \in(-0.2,1.0)$. Each black dot represents a non-trivial numerical solution, which we found only inside the regions bounded by the dotted lines. In the light grey region, within our numerical accuracy we could not determine whether the non-trivial solutions were thermodynamically preferred. In the dark grey region, the non-trivial solutions were thermodynamically preferred, while in the white region they were not. In each phase diagram, a solid black line denotes the boundary between the dark grey and white regions.

specifically along the diagonal dotted line, non-trivial solutions only appear at $T / T_{\mathrm{K}}$ below the maximal critical value.

In each phase diagram in fig. 5.1, we have divided the region bounded by the dotted lines into three sub-regions, coded by shading: light grey, dark grey, and white, with a solid black line separating the dark grey and white regions. In the light grey regions, we found non-trivial solutions, but $|\Delta \mathcal{F}|$ was smaller than our numerical accuracy threshold of $10^{-4}$, hence we could not conclude whether the non-trivial solutions were thermodynamically preferred over the trivial solution. In the dark grey region, the 
non-trivial solutions had $|\Delta \mathcal{F}|>10^{-4}$ and $\Delta \mathcal{F}>0$, so the non-trivial solutions were thermodynamically preferred over the trivial solution. In the white region, $|\Delta \mathcal{F}|>10^{-4}$ but $\Delta \mathcal{F}<0$, so the trivial solution was thermodynamically preferred.

The solid black line separating the dark grey and white regions is an interpolation between thermodynamically preferred solutions: to the left of that line, towards smaller $\lambda /\left(2 \pi T_{\mathrm{K}}\right)$, the next nearest numerical solution that we obtained was not thermodynamically preferred. The actual boundary between thermodynamically favoured and dis-favoured solutions is thus either at the solid black line, or somewhere between the solid black line and the first black dots to its left.

In any of the phase diagrams in fig. 5.1, imagine moving down along a vertical line in the phase diagram. Our results demonstrate that for sufficiently large AFM $\lambda /\left(2 \pi T_{\mathrm{K}}\right)$ a phase transition will occur, from the trivial state with no Kondo screening and zero spin-spin correlations to the non-trivial state with Kondo screening and non-zero AFM spin-spin correlations of order $N^{2}$. As discussed in subsection 5.5.1, in our model and with our ansatz, these are the only two possible states.

The order of these phase transitions depends on $\lambda /\left(2 \pi T_{\mathrm{K}}\right)$. For example, in any of the phase diagrams in fig. 5.1, suppose we move down along a vertical line such that we hit the diagonal dotted line. In that case, as we reduce $T / T_{\mathrm{K}}$ non-trivial solutions appear at the diagonal dotted line, but are not thermodynamically preferred. The nontrivial solutions become thermodynamically preferred only at the critical $T / T_{\mathrm{K}}$ where our vertical line hits the dark grey region. As a result, as $T / T_{\mathrm{K}}$ drops below the critical $T / T_{\mathrm{K}}$ all one-point functions will jump from zero to non-zero values, indicating a firstorder transition. Suppose we then increase $\lambda /\left(2 \pi T_{\mathrm{K}}\right)$ and repeat the process, such that now as we reduce $T / T_{\mathrm{K}}$ we hit the horizontal dotted line. In that case, although we cannot say for certain due to our limited numerical accuracy, our numerical results are consistent with the non-trivial solutions being thermodynamically preferred as soon as they appear. In those cases, we expect the one-point functions to increase smoothly from zero, indicating a continuous transition.

Our results for the one-point functions are consistent with such an interpretation. Fig. 5.2 shows our numerical results for $\frac{\kappa}{4 N} \frac{\left\langle\left|\mathcal{O}_{\mathrm{I}}\right|\right\rangle}{\sqrt{T_{\mathrm{K}}}}=-\alpha_{T} \sqrt{2 \pi T / T_{\mathrm{K}}}$ as a function of $T / T_{\mathrm{K}}$ 


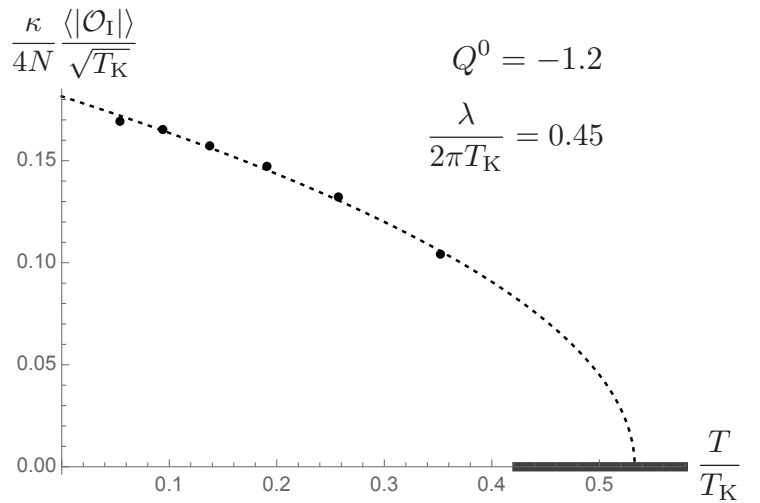

(a.)

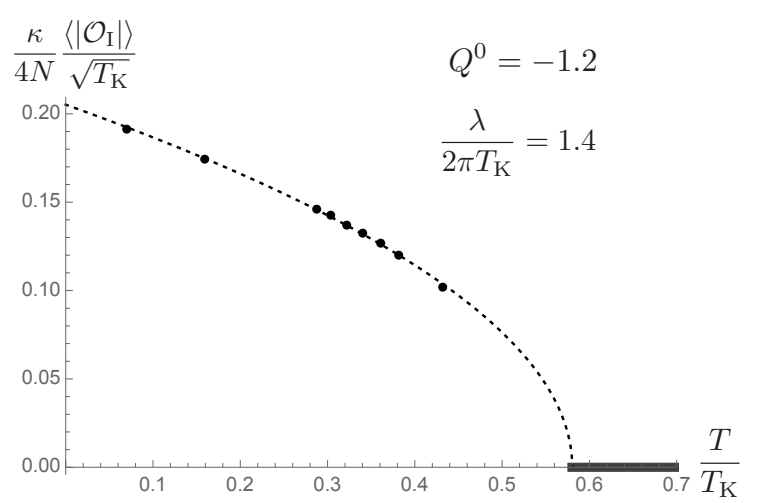

(b.)

Figure 5.2: Our numerical results for $\frac{\kappa}{4 N} \frac{\left\langle\left|\mathcal{O}_{\mathrm{I}}\right|\right\rangle}{\sqrt{T_{\mathrm{K}}}}=-\alpha_{T} \sqrt{2 \pi T / T_{\mathrm{K}}}$ as a function of $T / T_{\mathrm{K}}$ for $Q^{0}=-1.2$, with (a.) $\lambda /\left(2 \pi T_{\mathrm{K}}\right)=0.45$, and (b.) $\lambda /\left(2 \pi T_{\mathrm{K}}\right)=1.4$. In each figure, the black dots are our numerical data, the dotted line is a numerical fit to a secondorder mean-field transition with critical exponent $1 / 2$, and the heavy grey line segment at $\left\langle\left|\mathcal{O}_{\mathrm{I}}\right|\right\rangle=0$ represents the trivial solution where thermodynamically preferred. As we decrease $T / T_{\mathrm{K}}$ a phase transition occurs approximately where the heavy grey line segment ends. Our results suggest a first-order transition in (a.) and a second-order mean-field transition in (b.), consistent with our expectations from fig. 5.1 (c.).

for $Q^{0}=-1.2$, with $\lambda /\left(2 \pi T_{\mathrm{K}}\right)=0.45$ in fig. 5.2 (a.) and $\lambda /\left(2 \pi T_{\mathrm{K}}\right)=1.4$ in fig. 5.2 (b.). In each figure, each black dot represents a non-trivial numerical solution, the dotted curve is a numerical fit to our data in the form of a second-order mean-field transition with critical exponent $1 / 2$, and the heavy grey line segment at $\left\langle\left|\mathcal{O}_{\mathrm{I}}\right|\right\rangle=0$ represents the trivial solution for $T / T_{\mathrm{K}}$ values where the non-trivial solution is preferred. In other words, in fig. 5.2 (a.) the heavy grey line segment extends to the value of $T / T_{\mathrm{K}}$ of the solid black line at $\lambda /\left(2 \pi T_{\mathrm{K}}\right)=0.45$ in fig. 5.1 (c.), while in fig. 5.2 (b.) the heavy grey line segment extends down to the $T / T_{\mathrm{K}}$ value of the horizontal dotted line in fig. 5.1 (c.). For $\lambda /\left(2 \pi T_{\mathrm{K}}\right)=0.45$, the phase diagram in fig. 5.1 (c.) suggests a first-order transition, and indeed fig. 5.2 (a.) suggests that the transition cannot be continuous: the fit to our data suggests that $\left\langle\left|\mathcal{O}_{\mathrm{I}}\right|\right\rangle$ jumps from $\left\langle\left|\mathcal{O}_{\mathrm{I}}\right|\right\rangle=0$ to $\left\langle\left|\mathcal{O}_{\mathrm{I}}\right|\right\rangle \neq 0$ when the transition occurs. On the other hand, for $\lambda /\left(2 \pi T_{\mathrm{K}}\right)=1.4$, the phase diagram in fig. 5.1 (c.) suggests a continuous transition as indeed implied by fig. 5.2 (b.): the fit to our data suggests that $\left\langle\left|\mathcal{O}_{\mathrm{I}}\right|\right\rangle$ rises smoothly from zero starting at the transition with second-order mean-field exponent. 


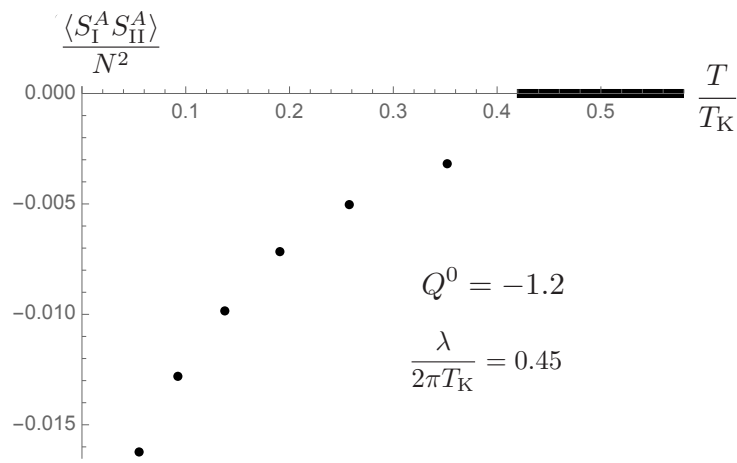

(a.)

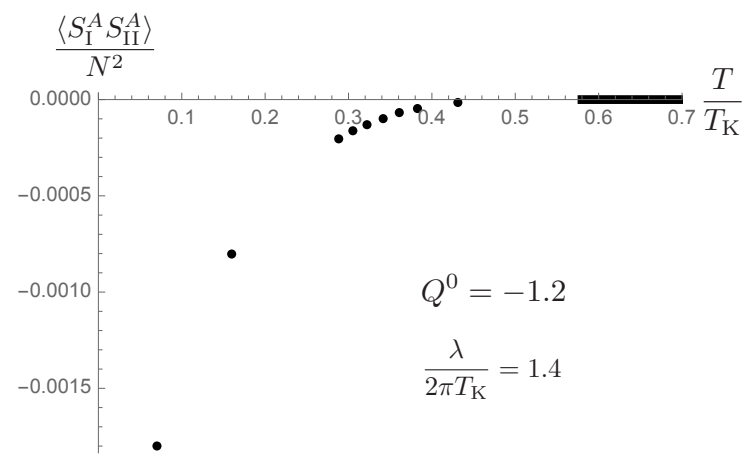

(b.)

Figure 5.3: Our numerical results for $\left\langle S_{\mathrm{I}}^{A} S_{\mathrm{II}}^{A}\right\rangle / N^{2}=-\mathcal{Q}^{2} / 2$ as a function of $T / T_{\mathrm{K}}$ for $Q^{0}=-1.2$, with (a.) $\lambda /\left(2 \pi T_{\mathrm{K}}\right)=0.45$ and (b.) $\lambda /\left(2 \pi T_{\mathrm{K}}\right)=1.4$. In each figure, the black dots are our numerical data and the heavy grey line segment at $\left\langle S_{\mathrm{I}}^{A} S_{\mathrm{II}}^{A}\right\rangle / N^{2}=0$ represents the trivial solution where thermodynamically preferred. As we decrease $T / T_{\mathrm{K}}$ a phase transition occurs approximately where the heavy grey line segment ends. Our results suggest a first-order transition in (a.) and a continuous transition in (b.), consistent with our expectations from fig. 5.1 (c.).

Fig. 5.3 shows our numerical results for the order $N^{2}$ contribution to the spinspin correlator (5.59), $\left\langle S_{\mathrm{I}}^{A} S_{\mathrm{II}}^{A}\right\rangle / N^{2}=-\mathcal{Q}^{2} / 2$, as a function of $T / T_{\mathrm{K}}$ for $Q^{0}=-1.2$, with $\lambda /\left(2 \pi T_{\mathrm{K}}\right)=0.45$ in fig. 5.3 (a.) and $\lambda /\left(2 \pi T_{\mathrm{K}}\right)=1.4$ in fig. 5.3 (b.). As in fig. 5.2, the black dots in fig. 5.3 represent non-trivial numerical solutions and the heavy grey line represents the trivial solution with $\left\langle S_{\mathrm{I}}^{A} S_{\mathrm{II}}^{A}\right\rangle / N^{2}=0$ for $T / T_{\mathrm{K}}$ values where the trivial solution is thermodynamically preferred. Our numerical results in fig. 5.3 (a.) for $\lambda /\left(2 \pi T_{\mathrm{K}}\right)=0.45$ suggest that $\left\langle S_{\mathrm{I}}^{A} S_{\mathrm{II}}^{A}\right\rangle / N^{2}$ jumps from $\left\langle S_{\mathrm{I}}^{A} S_{\mathrm{II}}^{A}\right\rangle / N^{2}=0$ to $\left\langle S_{\mathrm{I}}^{A} S_{\mathrm{II}}^{A}\right\rangle / N^{2} \neq 0$ when the transition occurs, consistent with a first-order transition, while fig. 5.3 (b.) for $\lambda /\left(2 \pi T_{\mathrm{K}}\right)=1.4$ suggests that $\left\langle S_{\mathrm{I}}^{A} S_{\mathrm{II}}^{A}\right\rangle / N^{2}$ rises smoothly from zero through the transition, consistent with a continuous transition. These results conform to our expectations from the corresponding phase diagram, fig. 5.1 (c.).

Most importantly, the phase diagrams in fig. 5.1 strongly suggest that in our model a quantum phase transition occurs as a function of increasing $\lambda /\left(2 \pi T_{\mathrm{K}}\right)$, from the trivial state to the non-trivial state. Moreover, fig. 5.1 suggests that such putative quantum phase transitions occur at non-zero AFM values of $\lambda /\left(2 \pi T_{\mathrm{K}}\right)$. For example, when $Q^{0}=-1$, fig. 5.1 (b.) suggests that a transition may occur approximately where the solid black line hits the horizontal axis, $\lambda /\left(2 \pi T_{\mathrm{K}}\right) \approx 0.4$. The putative quantum 
phase transitions in our model also appear to be first-order: as we increase $\lambda /\left(2 \pi T_{\mathrm{K}}\right)$ through the critical value, all one-point functions and the phase shift will jump from zero (white region) to non-zero values (dark grey region). Similarly, the quantum phase transition in the original large- $N$ two-impurity Kondo model of refs. [224,225] occurred at a non-zero AFM value of $\lambda_{\mathrm{RKKY}} / T_{\mathrm{K}}$ and was first-order.

However, we were unable to obtain non-trivial numerical solutions at exactly $T=0$. In general, as $T / T_{\mathrm{K}}$ decreases our numerical solutions for $\phi$ tend to grow, apparently without bound. Such growth is typical for scalar fields in the probe limit when the scalar potential includes only a mass term, see for example ref. [288]. Most likely, obtaining reliable numerical solutions at $T=0$ will require leaving the probe limit, i.e. including the back-reaction of the matter fields on the metric. For the holographic single-impurity Kondo model of ref. [18] this was studied in ref. [289]. We leave the analogous study for our model and the fate of our model at $T=0$ for future research.

\subsection{Holographic Kondo and Fano resonances}

In this section we briefly summarise our results from refs. $[16,17]$ on correlators in the holographic single-impurity Kondo model from ref. [18].

As mentioned in section 5.3, we recover the single-impurity from the two-impurity model if we only retain the $U(1)$ component of the YM field, and correspondingly only a single component of the scalar doublet. More precisely, we obtain the singleimpurity action from the two-impurity action $(5.13 \mathrm{c})$ if we let $\left(\Phi_{\mathrm{I}}, \Phi_{\mathrm{II}}\right)^{\mathrm{T}} \rightarrow(\Phi, \Phi)^{\mathrm{T}}$, $a_{m}^{a} \tau^{a} \rightarrow a_{m} \mathbb{1}, \operatorname{tr}\left(f^{m n} f_{m n}\right) \rightarrow f^{m n} f_{m n}$ with $f=\mathrm{d} a$, and divide the action by a factor of two.

We continue to work in a radial gauge, $a_{z}=0$. For static background solutions, conservation of the $U(1)$ current requires that the phase of the complex scalar $\Phi$ be a constant, which we can set to zero with a residual $U(1)$ gauge transformation. The background equations of motion for $\phi(z) \equiv|\Phi(z)|$ and $a_{t}(z)$ are then the same as eqs. (5.32b)-(5.32c) with $\mathcal{A}_{t}^{+} \rightarrow a_{t}(z)$. As before, we fix the leading mode $Q$ in $a_{t}$ 's near-boundary expansion, $a_{t}(z)=Q / z+\ldots$, to fix the size of the dual impurity. Next recall that the operator $\mathcal{O}$ dual to $\Phi$ must have dimension $1 / 2$ so that the Kondo 
interaction $-\frac{1}{2} \lambda_{\mathrm{K}} \mathcal{O}^{\dagger} \mathcal{O}$ is classically marginal. The near-boundary expansions of $\Phi$ and $\Phi^{\dagger}$ must therefore take the form

$$
\Phi=\alpha \sqrt{z} \log z+\beta+\ldots, \quad \Phi^{\dagger}=\alpha^{\dagger} \sqrt{z} \log z+\beta^{\dagger}+\ldots
$$

which requires choosing $M^{2}$ such that $\Phi^{\prime} s$ effective mass is $M^{2}-Q^{2}=-1 / 4$, as discussed in section 5.4. In this section, $\alpha$ and $\beta$ denote the complex modes of the complex field $\Phi$, unlike in the previous sections, where they referred to the real modes of the real field $\phi$. Accordingly, in the single-impurity case the variation of the undeformed renormalised on-shell action gives [17]

$$
\delta S_{\text {ren }}=-N \int \mathrm{d} t\left(\beta^{\dagger} \delta \alpha+\beta \delta \alpha^{\dagger}\right) \quad \Longrightarrow \quad\langle\mathcal{O}\rangle=-N \beta^{\dagger}, \quad\left\langle\mathcal{O}^{\dagger}\right\rangle=-N \beta,
$$

analogous to the two-impurity result (5.40), which had $\delta \alpha=\delta \alpha^{\dagger}$ and $\beta=\beta^{\dagger}$. Likewise, the Kondo double-trace deformation becomes $S_{\mathrm{K}}=N \kappa \int \mathrm{d} t \beta^{\dagger} \beta$ with holographic Kondo coupling $\kappa$, analogous to eq. (5.46). The Kondo interaction $S_{\mathrm{K}}$ leaves $\langle\mathcal{O}\rangle$ and $\left\langle\mathcal{O}^{\dagger}\right\rangle$ invariant, but it changes the sources of $\mathcal{O}$ and $\mathcal{O}^{\dagger}$ to $\alpha-\kappa \beta$ and $\alpha^{\dagger}-\kappa \beta^{\dagger}$ respectively. States that include the Kondo double-trace deformation but no single-trace deformation are thus described by bulk solutions that satisfy the boundary conditions $\kappa=\alpha / \beta=\alpha^{\dagger} / \beta^{\dagger}$. The dependence of $\langle\mathcal{O}\rangle,\left\langle\mathcal{O}^{\dagger}\right\rangle$ on variations of the sources $\alpha-\kappa \beta$, $\alpha^{\dagger}-\kappa \beta^{\dagger}$ is captured by the retarded correlators, as explained in chapter 3:

$$
\begin{gathered}
\delta\langle\mathcal{O}(\omega)\rangle=-G_{\mathcal{O O}}(\omega) \delta(\alpha-\kappa \beta)-G_{\mathcal{O O}^{\dagger}}(\omega) \delta\left(\alpha^{\dagger}-\kappa \beta^{\dagger}\right) \\
\delta\left\langle\mathcal{O}^{\dagger}(\omega)\right\rangle=-G_{\mathcal{O}^{\dagger} \mathcal{O}}(\omega) \delta(\alpha-\kappa \beta)-G_{\mathcal{O}^{\dagger} \mathcal{O}^{\dagger}}(\omega) \delta\left(\alpha^{\dagger}-\kappa \beta^{\dagger}\right) .
\end{gathered}
$$

In order to compute these correlators we need to consider fluctuations $\delta \Phi(t), \delta \Phi^{\dagger}(t)$ of $\Phi, \Phi^{\dagger}$. Linearising the equations of motion around a static background solution $\phi(z)$, $a_{t}(z)$ and Fourier transforming via $\partial_{t} \rightarrow-i \omega$, we find that $\delta \Phi(\omega), \delta \Phi^{\dagger}(\omega)$ obey

$$
\begin{gathered}
\partial_{z}\left(\sqrt{-g} g^{z z} \partial_{z} \delta \Phi\right)+\left[\frac{\left(\omega+a_{t}\right)^{2}}{h}-\frac{M^{2}}{z^{2}}\right] \delta \Phi=-\frac{\left(\omega+2 a_{t}\right)}{h} \phi \delta a_{t}, \\
\partial_{z}\left(\sqrt{-g} g^{z z} \partial_{z} \delta \Phi^{\dagger}\right)+\left[\frac{\left(-\omega+a_{t}\right)^{2}}{h}-\frac{M^{2}}{z^{2}}\right] \delta \Phi^{\dagger}=-\frac{\left(-\omega+2 a_{t}\right)}{h} \phi \delta a_{t} .
\end{gathered}
$$

We will not need the equation of motion satisfied by a fluctuation $\delta a_{t}$ of the gauge field in the following discussion. In order to obtain the retarded response of the system we impose incoming-wave boundary conditions on $\delta \Phi, \delta \Phi^{\dagger}$ at the horizon $z=z_{H}[47,73]$. 
Single-impurity phase diagram: The phase diagram of the holographic singleimpurity model, studied in ref. [18], depends on a single dimensionless parameter, $T / T_{\mathrm{K}}$, which is related to the holographic Kondo coupling via eq. (5.72). At high temperatures $T / T_{\mathrm{K}}$, only the trivial solution $a_{t}(z)=Q\left(1 / z-1 / z_{H}\right), \phi(z)=0$ exists, dual to a trivial state without Kondo screening, $\langle\mathcal{O}\rangle=0$. The trivial solution becomes unstable at a critical temperature $T_{c} / T_{\mathrm{K}}$, and non-trivial solutions with $\phi(z) \neq 0$ are thermodynamically preferred below $T_{c} / T_{\mathrm{K}}$ : $\langle O\rangle$ condenses in a second-order mean-field transition to a low-temperature phase with Kondo screening and a phase shift.

Correlators in the unscreened phase: The equations of motion (5.79) for $\delta \Phi$ and $\delta \Phi^{\dagger}$ decouple in the unscreened phase $\phi=0, a_{t}=Q\left(1 / z-1 / z_{H}\right)$, and can in fact be solved exactly in terms of hypergeometric functions. ${ }^{17}$ In particular, the solution fixes the sub-leading modes $\delta \beta, \delta \beta^{\dagger}$ in terms of the leading modes $\delta \alpha, \delta \alpha^{\dagger}$ :

$$
\begin{array}{r}
\delta \beta=R(\omega ; Q) \delta \alpha, \quad \delta \beta^{\dagger}=R(\omega ;-Q) \delta \alpha^{\dagger}, \\
R(\omega, Q) \equiv H\left(-\frac{1}{2}+i Q-i \omega z_{H}\right)+H\left(-\frac{1}{2}-i Q\right)-\ln \left(\frac{z_{H}}{2}\right) .
\end{array}
$$

Here, $H(n)$ denotes the $n$-th harmonic number and the argument of the logarithm is made dimensionless by the unit AdS radius. The decoupling of $\delta \Phi$ and $\delta \Phi^{\dagger}$ implies that variations of the one-point functions, eqs. (5.77) and (5.78), become

$$
\begin{aligned}
& \delta\langle\mathcal{O}\rangle=-N \delta \beta^{\dagger}=-N \delta \alpha^{\dagger} \frac{\delta \beta^{\dagger}}{\delta \alpha^{\dagger}}=-G_{\mathcal{O O}^{\dagger}} \delta \alpha^{\dagger}\left(1-\kappa \frac{\delta \beta^{\dagger}}{\delta \alpha^{\dagger}}\right), \\
& \delta\left\langle\mathcal{O}^{\dagger}\right\rangle=-N \delta \beta=-N \delta \alpha \frac{\delta \beta}{\delta \alpha}=-G_{\mathcal{O}^{\dagger} \mathcal{O}} \delta \alpha\left(1-\kappa \frac{\delta \beta}{\delta \alpha}\right)
\end{aligned}
$$

from which we obtain $G_{\mathcal{O}^{\dagger} \mathcal{O}^{\dagger}}=G_{\mathcal{O O}}=0$ and

$$
G_{\mathcal{O}^{\dagger} \mathcal{O}}=N \frac{\delta \beta / \delta \alpha}{1-\kappa \delta \beta / \delta \alpha}, \quad G_{\mathcal{O O}^{\dagger}}=N \frac{\delta \beta^{\dagger} / \delta \alpha^{\dagger}}{1-\kappa \delta \beta^{\dagger} / \delta \alpha^{\dagger}}
$$

Inserting the result (5.80) and using eqs. (5.71)-(5.72), we can re-write eq. (5.82) as

$$
\begin{aligned}
& G_{\mathcal{O}^{\dagger} \mathcal{O}}=-\frac{N}{\kappa}\left(1+\frac{1}{\kappa D(\omega ; Q)}\right), \quad G_{\mathcal{O O}^{\dagger}}=-\frac{N}{\kappa}\left(1+\frac{1}{\kappa D(\omega ;-Q)}\right) \\
& D(\omega ; Q) \equiv H\left(-\frac{1}{2}+i Q-i \frac{\omega}{2 \pi T}\right)+H\left(-\frac{1}{2}-i Q\right)+\ln \left(\frac{2 T}{T_{\mathrm{K}}}\right) .
\end{aligned}
$$

\footnotetext{
${ }^{17}$ The explicit solutions can be found in eqs. (3.23)-(3.27) in ref. [17], where $\delta \Phi$ and $\delta \Phi^{\dagger}$ are referred to as $y_{+}$and $y_{-}$respectively.
} 
The correlators (5.82) have poles located at the quasi-normal modes of the scalar, that is, at the eigenfrequencies of incoming-wave solutions to eqs. (5.79) which satisfy the appropriate boundary conditions $\kappa=\delta \alpha / \delta \beta=\delta \alpha^{\dagger} / \delta \beta^{\dagger}$. Because $D(\omega ;-Q)=$ $D\left(-\omega^{*} ; Q\right)^{*}$, the poles of $G_{\mathcal{O}^{\dagger} \mathcal{O}}$ and $G_{\mathcal{O O}^{\dagger}}$ come in pairs mirrored about the imaginary axis in the complex $\omega$-plane. At sufficiently high $T$, we find that all quasi-normal modes lie in the lower half of the complex plane, demonstrating the stability of the unscreened phase [16]. As we lower $T$, the quasi-normal modes move closer to the real axis, until the two lowest-lying modes cross the real axis at $T_{c}$, indicating the onset of the phase transition to the screened phase. From eq. (5.83), we find that the two lowest-lying poles meet at the origin $\omega=0$ at $T=T_{c}$ and obtain an exact expression for $T_{c}$ :

$$
T_{c}=\frac{1}{2} T_{\mathrm{K}} \exp \left(-2 \operatorname{Re}\left[H\left(-\frac{1}{2}+i Q\right)\right]\right) .
$$

The spectral functions $\rho_{\mathcal{O}^{\dagger} \mathcal{O}}(\omega) \equiv-2 \operatorname{Im}\left(G_{\mathcal{O}^{\dagger} \mathcal{O}}\right), \rho_{\mathcal{O O}^{\dagger}}(\omega) \equiv-2 \operatorname{Im}\left(G_{\mathcal{O O}^{\dagger}}\right)$ with $\omega$ real are dominated by the lowest-lying poles $\omega_{ \pm}$of the correlators (5.83). Crucially, the corresponding residues $Z_{ \pm}$are complex. For instance, near $T=T_{c}$, expanding $D(\omega ; Q)$ for small $\omega / T$ shows that $G_{\mathcal{O}^{\dagger} \mathcal{O}} \sim Z_{+} /\left(\omega-\omega_{+}\right)$and $G_{\mathcal{O O}^{\dagger}} \sim Z_{-} /\left(\omega-\omega_{-}\right)$with

$$
Z_{ \pm}=-i \frac{N}{\kappa^{2}} \frac{2 \pi T_{c}}{H^{\prime}(-1 / 2 \pm i Q)}, \quad \omega_{ \pm}=-i \frac{2 \pi\left(T-T_{c}\right)}{H^{\prime}(-1 / 2 \pm i Q)}
$$

to leading order in $T / T_{c}-1$. Following our discussion in subsection 3.4.3, the fact that the residues are complex and not real implies that the spectral functions in the unscreened phase display asymmetric Fano resonances. In particular, unless $Q=0$, in which case $Z_{ \pm}$become purely imaginary, $\rho_{\mathcal{O}^{\dagger} \mathcal{O}}$ and $\rho_{\mathcal{O O}^{\dagger}}$ are not anti-symmetric under $\omega \rightarrow-\omega$, i.e. they break particle-hole symmetry.

Correlators in the screened phase: In the screened phase $\phi \neq 0$, the equations (5.79) for $\delta \Phi$ and $\delta \Phi^{\dagger}$ are coupled. However, in this case fluctuations of the scalar's phase $\delta \psi$ conspire with fluctuations $\delta a_{t}$ of the gauge field to form the gauge invariant combination $\delta a_{t}+i \omega \delta \psi$. Because we do not want to change the impurity's representation we demand that fluctuations of the gauge field vanish, hence $\delta a_{t}+i \omega \delta \psi=0$. We are therefore only left with fluctuations of the modulus $\delta \phi \equiv \delta|\Phi|$. As a result, 
variations of the one-point functions (5.77) take the form

$$
\delta\langle\mathcal{O}\rangle=-N \delta \beta^{\dagger}=-N \frac{\delta|\beta|}{\delta|\alpha|} \delta|\alpha|, \quad \delta\left\langle\mathcal{O}^{\dagger}\right\rangle=-N \delta \beta=-N \frac{\delta|\beta|}{\delta|\alpha|} \delta|\alpha| .
$$

Comparing this to eq. (5.78) shows that in the screened phase

$$
G \equiv G_{\mathcal{O O}}=G_{\mathcal{O O}^{\dagger}}=G_{\mathcal{O}^{\dagger} \mathcal{O}}=G_{\mathcal{O} \dagger \mathcal{O} \dagger}=\frac{N}{2} \frac{\delta|\beta| / \delta|\alpha|}{1-\kappa \delta|\beta| / \delta|\alpha|}
$$

We have only been able to find numerical solutions for $\delta \phi$ and $\delta|\beta| / \delta|\alpha|$ in refs $[16,17]$.

Eq. (5.79) shows that the equation of motion for $\delta \phi=\delta \Phi+\delta \Phi^{\dagger}$ is invariant under $\omega \rightarrow-\omega$. We expect solutions to $\delta|\beta|$ for given $\delta|\alpha|$ to respect this symmetry and be either even or odd under $\omega \rightarrow-\omega$. However, $\delta|\beta| / \delta|\alpha|$ cannot be an even function of $\omega$ as in that case the spectral function $\rho \equiv-2 \operatorname{Im}(G)$ would violate the positivity property $\omega \rho(\omega) \geq 0 .{ }^{18}$ We therefore expect $\delta|\beta| / \delta|\alpha|$ to be an odd function of $\omega$, leading to an anti-symmetric spectral function. This is indeed confirmed by our numerical analysis in refs. [16,17]. At $T=T_{c}$, the poles of $G$ in the screened phase coincide with the quasi-normal modes in the unscreened phase. In particular, the lowest-lying pole $\omega_{*}$ sits at the origin at $T=T_{c}$. As we lower $T$ below $T_{c}$, the poles of $G$ move down in the complex plane. In particular, $\omega_{*}$ moves straight down the imaginary axis and grows as $\omega_{*} \propto-i\langle\mathcal{O}\rangle^{2}$ as $\langle\mathcal{O}\rangle$ condenses. This is a characteristic feature of the Kondo resonance at large $N$ [290], demonstrating that our holographic model realises a genuine Kondo effect. Moreover, the Kondo resonance appears in $\rho$ as an anti-symmetric Fano resonance and particle-hole symmetry, $\rho(-\omega)=-\rho(\omega)$, is restored for $T \leq T_{c}$.

Origin of Fano resonances: As reviewed in subsection 3.4.3, a Fano resonance arises when a Lorentzian resonance is immersed in a continuum of energy states. In our Kondo model, $\mathcal{O}$ 's spectrum inherits scale invariance from the UV fixed point and is therefore continuous. The marginally relevant Kondo coupling then triggers an RG flow, breaks scale invariance, and produces a resonance. In $(0+1)$ dimensions, the resonance cannot escape the continuum and hence must lead to a Fano resonance. This mechanism, to our knowledge new, predicts Fano resonances in any RG flow between $(0+1)$ dimensional fixed points such as Sachdev-Ye-Kitaev fixed points [291-294].

\footnotetext{
${ }^{18}$ We discuss the positivity property satisfied by spectral functions of non-Hermitian operators such as our $\mathcal{O}$ in appendix A.
} 


\subsection{Summary and outlook}

This chapter investigated inter-impurity interactions and correlation functions in a holographic Kondo model.

In section 5.2, we reviewed the single- and two-impurity Kondo models. The singleimpurity model consists of free chiral fermions $\psi$ in $(1+1)$ dimensions (representing electron $s$-waves), coupled to an impurity spin $S$ at the origin. Impurities in totally anti-symmetric representations of the $S U(N)$ spin group admit a description in terms of Abrikosov pseudo-fermions $\chi$, which introduces an auxiliary $U(1)$ acting on $\chi$. At large $N$, the anti-ferromagnetic Kondo interaction between $\psi$ and $S$ takes the form of a marginally relevant double-trace deformation of the free UV fixed point. This induces a $(0+1)$-dimensional $\mathrm{RG}$ flow at the impurity. The large- $N$ Kondo effect appears as condensation of $\mathcal{O}=\psi^{\dagger} \chi$ below a critical temperature $T_{c}$, indicating the screening of the impurity, $\chi$, by the electrons, $\psi$. The operator $\mathcal{O}$ is invariant under the $S U(N)$ spin group, but is charged under both the electromagnetic $U(1)$ of the chiral $\psi$ and under the auxiliary $U(1)$ of the $\chi$. The two-impurity model admits a similar description at sufficiently large distances, where the impurity separation can be ignored. In that case, the $\psi$ interact with two identical impurities at the origin. The two impurity spins introduce two flavours of pseudo-fermions and the auxiliary $U(1)$ is enhanced to $U(2)$. Moreover, the impurities are coupled to each other by the Heisenberg-type RKKY interaction. At large $N$, the RKKY interaction takes the form of a relevant double-trace deformation, eq. (5.10). This is the first main result of this chapter and is valid independently of a holographic description.

Section 5.3 introduced our holographic two-impurity model, extending the holographic single-impurity model from ref. [18]. In order to obtain a holographic description, we effectively replaced the free UV fixed point of the original Kondo model with a strongly coupled holographic CFT. More precisely, we gauged the $S U(N)$ spin group, which introduced additional adjoint degrees of freedom, and took the limits of large $N$ and strong coupling, producing a holographic CFT with a gravity dual in $A d S_{3}$. Our holographic bottom-up model contains the $A d S_{3}$ metric, dual to the CFT stress-tensor, and a $U(1)$ Chern-Simons field $A$, dual to the $U(1)$ current of the chiral $\psi$. On an $A d S_{2}$ 
defect at the impurity location, our model further contains a $U(2)$ Yang-Mills field $a$, dual to the $U(2)$ current of the $\chi$, and a charged scalar $\Phi$, dual to the charged scalar operator $\mathcal{O}$. We employed a probe limit and ignored the backreaction of the fundamental degrees of freedom $A, a, \mathcal{O}$, whose action scales linearly in $N$, on the AdS metric, whose action scales quadratically in $N$.

In section 5.4, we identified the Kondo coupling and the RKKY inter-impurity coupling as boundary conditions on $\Phi$ and $a$ respectively. This was the first identification of an inter-impurity coupling in holography and constitutes our second main result.

Section 5.5 discussed the two-impurity phase diagram. We showed in subsection 5.5.2 that ferromagnetic correlations between totally anti-symmetric $S U(N)$ spins scale at most linearly in $N$ and, at large $N$, are suppressed compared to anti-ferromagnetic correlations which scale as $N^{2}$. This result is based only on $S U(N)$ representation theory and constitutes the third main finding of this chapter. It holds independently of holography and may have implications for other large- $N$ descriptions of magnetism. Because of the probe limit, our holographic model could thus only distinguish order $N^{2}$ anti-ferromagnetic spin-spin correlations from uncorrelated spins. In fact, our model was only able to describe two different phases: a trivial phase with uncorrelated spins and no Kondo screening, and a non-trivial phase with anti-ferromagnetic spin-spin correlations and simultaneous Kondo screening. Our numerical results in subsection 5.5.3 showed that only the trivial state exists for ferromagnetic RKKY coupling, while at sufficiently strong anti-ferromagnetic RKKY coupling a phase transition to the nontrivial phase occurs at low temperatures. Moreover, our numerical results suggest that the transition between the two phases persists at $T=0$ as a first-order quantum phase transition at a non-zero anti-ferromagnetic value of the RKKY coupling. This is the fourth main result of this chapter. Our phase diagram is consistent with field theory expectations. For one thing, the quantum phase transition in the large- $N$ two impurity Kondo model of refs. [224,225] also occurred at a non-zero AFM value of the RKKY coupling and was first-order. For another thing, the coexistence of Kondo and interimpurity screening is generic in two-impurity Kondo models and is believed to occur in the Kondo lattice too [184, 185, 236]. 
Section 5.6 summarised our results on holographic correlators of $\mathcal{O}$ in the singleimpurity case. We found that the spectrum of $\mathcal{O}$ exhibits a Fano resonance at all temperatures. In the high-temperature phase, where the impurity is unscreened, the Fano resonance is asymmetric and breaks particle-hole symmetry. In the low-temperature phase, where $\mathcal{O}$ condenses and the impurity is screened, particle-hole symmetry is restored and the Fano resonance can be identified with the Kondo resonance, caused by a frequency pole $\omega_{*} \propto-i\langle\mathcal{O}\rangle^{2}$ in $\mathcal{O}$ 's correlators. The identification of the Kondo resonance constitutes the fifth main result of this chapter. Fano resonances occur when a Lorentzian resonance, in our case produced by the marginally relevant Kondo coupling, is immersed in a continuum, in our case provided by the impurity's $(0+1)$-dimensional scale invariance in the UV. The sixth main result of this chapter is our proposal that, following the same mechanism, Fano resonances should be a generic feature of RG flows between $(0+1)$-dimensional fixed points.

The appearance of the Kondo resonance confirms that our holographic model indeed describes a strongly coupled Kondo model, as opposed to some other impurity physics, and demonstrates that the Kondo effect can survive strong correlations essentially intact. Moreover, the phase diagram of our holographic two-impurity model shows that holographic Kondo models can capture essential two-impurity Kondo phenomena, including most importantly a quantum phase transition, and can thus provide a foundation for future model-building. Of course, much work must still be done to reach the ultimate goal of building and solving a holographic Kondo lattice.

In particular, to describe a Kondo lattice we must separate the impurities in space. In the two-impurity case, this will break the auxiliary $U(2)$ down to $U(1) \times U(1)$ and will render the RKKY interaction non-local. The only way to couple the two separated impurity spins, then, is to connect them with a non-local gauge-invariant operator, i.e. with an open Wilson line of the gauged $S U(N)$ spin group. In holographic models, an open Wilson line is dual to an open string in the bulk [295].

However, separating the impurities in our holographic model would radically change the field theory interpretation. In particular, we could no longer interpret the dual field theory as a $(1+1)$-dimensional CFT description of a two-impurity Kondo system, since 
that description is based on the limit that the separation between the impurities is negligible. Instead, our model would be more similar to a genuinely $(1+1)$-dimensional Luttinger liquid coupled to two separated impurities. For separated Kondo impurities, and in particular for the Kondo lattice, a $(1+1)$-dimensional description must thus be abandoned. To build a holographic Kondo lattice one should commit to holographic models in which the field theory has two or more spatial dimensions, and introduce an infinite number of impurities. As mentioned in ref. [250] though, an infinite number of impurities renders the probe limit by definition invalid.

A different issue, which our model has in common with non-holographic large- $N$ Kondo models [207], is that the spin group $S U(N)$ makes it difficult to describe antiferromagnetic ordering at large $N$. The problem is that two impurity spins can only lock into a singlet of $S U(N)$ if they are in a representation whose Young tableau has exactly $N / 2$ boxes. An alternative is the symplectic large- $N$ limit: instead of replacing $S U(2)$ with $S U(N)$ and taking $N \rightarrow \infty$, the symplectic large- $N$ limit is based on identifying $S U(2) \simeq S p(1)$, replacing $S p(1)$ with $S p(N)$, and then taking $N \rightarrow \infty[296,297]$. The symplectic large- $N$ limit allows for two impurity spins in the fundamental representation to lock into a singlet and hence allows for a genuine antiferromagnetic phase. Top-down holographic duals of strongly coupled theories with symplectic gauge groups can be realised by introducing orientifolds in the bulk [4].

Perhaps the most fundamental problem with all holographic quantum impurity models to date [18,244-257], however, is that the spin group is gauged. Because holography only provides access to gauge-invariant operators, important quantities that are not spin singlets, such as the magnetic susceptibility, cannot be calculated. The obvious route to address this issue is to develop holographic quantum impurity models in which spin is a global symmetry. In that case the large- $N$ strongly coupled gauge theory sector would merely provide a classical gravity dual.

We believe that the long-term goal of solving a (holographic) Kondo lattice provides sufficient motivation to pursue solutions to all of the problems above, using our holographic model as a starting point. 


\section{Chapter 6}

\section{Conclusion}

This thesis investigated applications of the gauge/gravity duality to strongly interacting quantum systems. Chapter 2 motivated the duality's origin within string theory and reviewed the holographic dictionary, which translates between the gauge theory and its gravity dual. In particular, we described how the computation of real-time correlators in the strongly coupled gauge theory reduces to obtaining classical wave solutions in AdS gravity. Chapter 3 reviewed real-time correlators and spectral functions in quantum systems. We explained how transport coefficients such as viscosity or conductivity can be obtained from low-energy correlators, and we discussed the origin of Fano resonances.

Chapter 4 contained our results on second-order hydrodynamics in non-conformal fluids. We derived new Kubo formulae for five second-order transport coefficients in terms of three-point correlators of the stress-tensor, valid for any uncharged relativistic fluid in $(3+1)$ dimensions. We then applied these Kubo formulae to a large class of non-conformal holographic fluids at infinite coupling. We showed that a specific linear combination of second-order coefficients, $\tilde{H}=2 \eta \tau_{\pi}-2\left(\kappa-\kappa^{*}\right)-\lambda_{2}$, vanishes in this class of models. We found strong evidence that the Haack-Yarom identity, $H=$ $2 \eta \tau_{\pi}-4 \lambda_{1}-\lambda_{2}=0$, which had been known to hold in conformal holographic fluids at infinite coupling, continues to hold in holographic fluids without conformal symmetry: Within our class of models, we proved that $H$ vanishes when leading non-conformal corrections are taken into account, and we showed numerically that $H$ remains zero also beyond leading non-conformal deformations. Results for adiabatic fluids [177] and for the entropy current in conformal fluids [93] suggest that $\tilde{H}=0$ and $H=0$ may 
indicate the suppression of entropy production at strong coupling. It would be desirable to find a more general proof that $\tilde{H}=0$ and $H=0$ hold universally in non-conformal holographic fluids at infinite coupling, ideally bringing these second-order relations on an equal footing with the first-order relation $\eta / s=1 / 4 \pi$. First, one would need to identify which conditions the bulk matter stress tensor must satisfy to ensure that it decouples from the metric fluctuations which enter $\tilde{H}$ and $H$. Given that both $\tilde{H}$ and $H$ are independent of sound perturbations we expect the appropriate conditions to be satisfied by most ordinary bulk matter, though dilatons might pose a challenge. Next, one would have to solve for the relevant metric fluctuations on a general RG-flow geometry, repeating our steps in section 4.4 , hopefully to find $\tilde{H}=0$ and to recast $H$ as an integral over the background. In order to demonstrate that this integral vanishes, our experience with the proof of $H=0$ to linear order suggests it might be helpful to use the emblackening factor $f$ as radial coordinate throughout the whole calculation.

Chapter 5 presented our results on a holographic Kondo model, which described impurity spins coupled to strongly correlated charge carriers. We extended the existing holographic single-impurity model to a two-impurity Kondo model with doubletrace inter-impurity coupling. Using $S U(N)$ representation theory, we showed that ferromagnetic correlations between totally anti-symmetric $S U(N)$ spins are suppressed compared to anti-ferromagnetic ones at large $N$. Indeed, our model could only describe a trivial phase with uncorrelated spins and no Kondo screening, and a non-trivial phase with anti-ferromagnetic spin-spin correlations and simultaneous Kondo screening. Our numerical results suggest a first-order quantum phase transition between these two phases. We then computed correlators in the single-impurity case. The interference between a resonance, produced by the marginally relevant Kondo coupling, and a continuous spectrum, provided by the $(0+1)$-dimensional scale invariance of the impurity in the UV, resulted in a Fano resonance, which we identified at low temperatures with the Kondo resonance. We proposed that, following the same mechanism, Fano resonances should be a generic feature of RG flows between $(0+1)$-dimensional fixed points. As next step towards a holographic Kondo lattice we suggest building a higher-dimensional holographic model in which the impurities can be separated in space. 


\section{Appendix A}

\section{Some Properties of Causal Correlators}

In this appendix we will summarise a few useful properties of causal correlators, see e.g. refs. $[47,71,90]$. The retarded and advanced Green's functions of two operators $\mathcal{A}(t, \underline{x})$ and $\mathcal{B}\left(t^{\prime}, \underline{x}^{\prime}\right)$ are defined as

$$
G_{\mathcal{A}, \mathcal{B}}^{R}\left(x ; x^{\prime}\right)=-i \theta\left(t-t^{\prime}\right)\left\langle\left[\mathcal{A}(x), \mathcal{B}\left(x^{\prime}\right)\right]\right\rangle, \quad G_{\mathcal{A}, \mathcal{B}}^{A}\left(x ; x^{\prime}\right)=i \theta\left(t^{\prime}-t\right)\left\langle\left[\mathcal{A}(x), \mathcal{B}\left(x^{\prime}\right)\right]\right\rangle,
$$

respectively. From now on we will assume translational invariance, i.e. $G_{\mathcal{A}, \mathcal{B}}^{R / A}\left(x ; x^{\prime}\right)=$ $G_{\mathcal{A}, \mathcal{B}}^{R / A}\left(x-x^{\prime}\right)$. The Fourier transforms then satisfy

$$
G_{\mathcal{A}, \mathcal{B}}^{R}(-k)=G_{\mathcal{B}, \mathcal{A}}^{A}(k), \quad G_{\mathcal{A}, \mathcal{B}}^{R}(k)^{*}=G_{\mathcal{A}^{\dagger}, \mathcal{B}^{\dagger}}^{R}(-k)=G_{\mathcal{B}^{\dagger}, \mathcal{A}^{\dagger}}^{A}(k) .
$$

One can show [90] that correlators in a state with time-reversal symmetry satisfy

$$
G_{\mathcal{A}, \mathcal{B}}^{R}(\omega, \underline{k})=\eta_{\mathcal{A}} \eta_{\mathcal{B}} G_{\mathcal{B}^{\dagger}, \mathcal{A}^{\dagger}}^{R}(\omega,-\underline{k}),
$$

where $\eta_{\mathcal{A}}, \eta_{\mathcal{B}}= \pm 1$ are the time-reversal eigenvalues of $\mathcal{A}, \mathcal{B}$.

The spectral function is defined as

$$
\rho_{\mathcal{A}, \mathcal{B}}(k)=\int \frac{\mathrm{d}^{d} k}{(2 \pi)^{d}} e^{i k x}\langle[A(x), B(0)]\rangle=i\left(G_{\mathcal{A}, \mathcal{B}}^{R}(k)-G_{\mathcal{A}, \mathcal{B}}^{A}(k)\right) .
$$

Using properties (A.1), $\rho_{\mathcal{A}, \mathcal{B}}$ can be written as

$$
\rho_{\mathcal{A}, \mathcal{B}}(k)=i\left(G_{\mathcal{A}, \mathcal{B}}^{R}(k)-G_{\mathcal{B}^{\dagger}, \mathcal{A}^{\dagger}}^{R}(k)^{*}\right) .
$$

If all operators are Hermitian, $\mathcal{A}(x)^{\dagger}=\mathcal{A}(x), \mathcal{B}(x)^{\dagger}=\mathcal{B}(x)$, then the spectral function can be viewed as a matrix in the space of Hermitian operators and is given by $i$ times the anti-Hermitian part of the matrix $\left(G_{\mathcal{A}, \mathcal{B}}^{R}\right)$ :

$$
\rho_{\mathcal{A}, \mathcal{B}}(k)=i\left(G_{\mathcal{A}, \mathcal{B}}^{R}(k)-G_{\mathcal{B}, \mathcal{A}}^{R}(k)^{*}\right) .
$$


The positivity property (3.52) for a single Hermitian operator translates into the requirement that $\omega \rho_{\mathcal{A}, \mathcal{B}}(\omega, \underline{k})$ be a positive semi-definite matrix. This property can be deduced from the spectral representation of $\rho_{\mathcal{A}, \mathcal{B}}(k)[71]$.

Let us now consider the case of a single complex operator $\mathcal{O}$ like the one we encounter in chapter 5. We can build two Hermitian operators from $\mathcal{O}$ :

$$
\mathcal{R} \equiv \mathcal{O}+\mathcal{O}^{\dagger}, \quad \mathcal{I} \equiv i\left(\mathcal{O}-\mathcal{O}^{\dagger}\right)
$$

The (real) source term $\left(\mathcal{O} \Phi+\mathcal{O}^{\dagger} \Phi^{\dagger}\right)$ in the generating functional for $\mathcal{O}$ then reads $(\mathcal{R} \operatorname{Re} \Phi+\mathcal{I} \operatorname{Im} \Phi)$. Using the chain rule for functional derivatives, we can write a generic correlator $G$ of $\mathcal{O}, \mathcal{O}^{\dagger}$ in terms of correlators of the Hermitian operators $\mathcal{R}, \mathcal{I}$ :

$$
\begin{aligned}
& G_{\mathcal{O O}}=\frac{1}{4}\left(G_{\mathcal{R}, \mathcal{R}}-G_{\mathcal{I}, \mathcal{I}}-i\left(G_{\mathcal{R}, \mathcal{I}}+G_{\mathcal{I}, \mathcal{R}}\right)\right), \\
& G_{\mathcal{O}^{\dagger} \mathcal{O}^{\dagger}}=\frac{1}{4}\left(G_{\mathcal{R}, \mathcal{R}}-G_{\mathcal{I}, \mathcal{I}}+i\left(G_{\mathcal{R}, \mathcal{I}}+G_{\mathcal{I}, \mathcal{R}}\right)\right), \\
& G_{\mathcal{O O}^{\dagger}}=\frac{1}{4}\left(G_{\mathcal{R}, \mathcal{R}}+G_{\mathcal{I}, \mathcal{I}}+i\left(G_{\mathcal{R}, \mathcal{I}}-G_{\mathcal{I}, \mathcal{R}}\right)\right), \\
& G_{\mathcal{O}^{\dagger} \mathcal{O}}=\frac{1}{4}\left(G_{\mathcal{R}, \mathcal{R}}+G_{\mathcal{I}, \mathcal{I}}-i\left(G_{\mathcal{R}, \mathcal{I}}-G_{\mathcal{I}, \mathcal{R}}\right)\right) .
\end{aligned}
$$

In section 5.6 we find that in the normal phase of the holographic Kondo model $G_{\mathcal{O O}}^{R}=$ $G_{\mathcal{O}^{\dagger} \mathcal{O}^{\dagger}}^{R}=0$ so that $G_{\mathcal{I}, \mathcal{I}}^{R}=G_{\mathcal{R}, \mathcal{R}}^{R}$ and $G_{\mathcal{I}, \mathcal{R}}^{R}=-G_{\mathcal{R}, \mathcal{I}}^{R}$. The spectral functions $\rho$ in the space of Hermitian operators $\mathcal{R}, \mathcal{I}$,

$$
(\rho)=\left(\begin{array}{cc}
\rho_{\mathcal{R}, \mathcal{R}} & \rho_{\mathcal{R}, \mathcal{I}} \\
\rho_{\mathcal{I}, \mathcal{R}} & \rho_{\mathcal{I}, \mathcal{I}}
\end{array}\right)
$$

then become

$$
(\rho)=\left(\begin{array}{cc}
\rho_{\mathcal{R}, \mathcal{R}} & \rho_{\mathcal{R}, \mathcal{I}} \\
-\rho_{\mathcal{R}, \mathcal{I}} & \rho_{\mathcal{R}, \mathcal{R}}
\end{array}\right)
$$

with eigenvalues $\rho_{\mathcal{R}, \mathcal{R}}-i \rho_{\mathcal{R}, \mathcal{I}}=2 \rho_{\mathcal{O}^{\dagger} \mathcal{O}}$ and $\rho_{\mathcal{R}, \mathcal{R}}+i \rho_{\mathcal{R}, \mathcal{I}}=2 \rho_{\mathcal{O O}^{\dagger}}$. The positivity property of $\rho$, namely the requirement that $\omega \rho$ be positive semi-definite, thus becomes

$$
\omega \rho_{\mathcal{O}^{\dagger} \mathcal{O}}(\omega)=\omega\left(-2 \operatorname{Im} G_{\mathcal{O}^{\dagger} \mathcal{O}}^{R}(\omega)\right) \geq 0, \quad \omega \rho_{\mathcal{O O}^{\dagger}}(\omega)=\omega\left(-2 \operatorname{Im} G_{\mathcal{O O}^{\dagger}}^{R}(\omega)\right) \geq 0
$$

In the condensed phase of the holographic Kondo model we obtain $G_{\mathcal{O O}}^{R}=G_{\mathcal{O}^{\dagger} \mathcal{O}^{\dagger}}^{R}=$ $G_{\mathcal{O O}^{\dagger}}^{R}=G_{\mathcal{O}^{\dagger} \mathcal{O}}^{R}=G_{\mathcal{R}, \mathcal{R}}^{R}$. The positivity property is therefore simply

$$
\omega \rho_{\mathcal{O}^{\dagger} \mathcal{O}}(\omega)=\omega\left(-2 \operatorname{Im} G_{\mathcal{O}^{\dagger} \mathcal{O}}^{R}(\omega)\right) \geq 0 .
$$




\section{Appendix B}

\section{A Class of Non-Conformal Holographic Fluids}

\section{B.1 Second-order constitutive relations}

This appendix contains the explicit constitutive relations for the stress tensor of an uncharged relativistic fluid up to second order in the gradient expansion that were used to derive eqs. (4.9) and (4.12). We define the 4-velocity as the unit timelike eigenvector of $\left\langle T^{\mu \nu}\right\rangle$ and $\epsilon$ as the corresponding eigenvalue to all orders in the gradient expansion (Landau frame) $[90,91,93]$. We further define the projection to symmetric, traceless tensors that are transverse to the fluid motion,

$$
\Delta^{\mu \nu}(x) \equiv u^{\mu}(x) u^{\nu}(x)+g_{(0)}^{\mu \nu}(x), \quad A^{<\mu \nu>} \equiv \frac{1}{2} \Delta^{\mu \rho}\left(A_{\rho \sigma}+A_{\sigma \rho}\right) \Delta^{\sigma \nu}-\frac{1}{3} \Delta^{\mu \nu}\left(\Delta^{\sigma \rho} A_{\sigma \rho}\right)
$$

and we define the shear tensor $\sigma^{\mu \nu} \equiv 2 \nabla^{<\mu} u^{\nu>}$ and the vorticity tensor

$$
\Omega^{\mu \nu} \equiv \frac{1}{2} \Delta^{\mu \rho}\left(\nabla_{\rho} u_{\sigma}-\nabla_{\sigma} u_{\rho}\right) \Delta^{\sigma \nu} .
$$

The constitutive relation for $\left\langle T^{\mu \nu}\right\rangle$ can then be written as

$$
\left\langle T^{\mu \nu}(x)\right\rangle=\epsilon(x) u^{\mu}(x) u^{\nu}(x)+p(x) \Delta^{\mu \nu}(x)+\Pi_{\text {conf. }}^{\mu \nu}(x)+\Pi_{\text {non-conf. }}^{\mu \nu}(x)+\mathcal{O}\left(\partial^{3}\right)
$$

where

$$
\begin{aligned}
\Pi_{\text {conf. }}^{\mu \nu}(x) \equiv & -\eta \sigma^{\mu \nu} \\
& +\eta \tau_{\pi}\left[\left(u^{\lambda} \nabla_{\lambda} \sigma\right)^{<\mu \nu>}+\frac{1}{3} \sigma^{\mu \nu}(\nabla \cdot u)\right] \\
& +\kappa\left[R^{<\mu \nu>}-2 u_{\lambda} R^{\lambda<\mu \nu>\kappa} u_{\kappa}\right] \\
& +\lambda_{1} \sigma_{\lambda}{ }^{<\mu} \sigma^{\nu>\lambda}+\lambda_{2} \sigma_{\lambda}{ }^{<\mu} \Omega^{\nu>\lambda}-\lambda_{3} \Omega_{\lambda}{ }^{<\mu} \Omega^{\nu>\lambda}
\end{aligned}
$$


is present in conformal and non-conformal fluids and was first derived in ref. [10], and where

$$
\begin{aligned}
\Pi_{\text {non-conf. }}^{\mu \nu}(x) \equiv & -\zeta \Delta^{\mu \nu}(\nabla \cdot u) \\
& +\eta \tau_{\pi}^{*} \frac{1}{3} \sigma^{\mu \nu}(\nabla \cdot u)+\kappa^{*} 2 u_{\lambda} R^{\lambda<\mu \nu>\kappa} u_{\kappa}+\lambda_{4} \nabla^{<\mu} \log s \nabla^{\nu>} \log s \\
& +\left(\zeta \tau_{\Pi} u^{\lambda} \nabla_{\lambda}(\nabla \cdot u)+\xi_{1} \sigma^{\kappa \lambda} \sigma_{\kappa \lambda}+\xi_{2}(\nabla \cdot u)^{2}+\xi_{3} \Omega^{\kappa \lambda} \Omega_{\kappa \lambda}\right. \\
& \left.+\xi_{4} \Delta_{\kappa}{ }^{\lambda}\left(\nabla_{\lambda} \log s\right) \Delta^{\kappa \rho}\left(\nabla_{\rho} \log s\right)+\xi_{5} R+\xi_{6} u^{\kappa} u^{\lambda} R_{\kappa \lambda}\right) \Delta^{\mu \nu}
\end{aligned}
$$

was constructed in ref. [93] and vanishes for conformal fluids. Transport coefficients entering $H$ and $\tilde{H}$, eqs. (4.1) and (4.75), are highlighted in blue in eqs (B.2)-(B.3).

\section{B.2 Sub-leading modes of metric perturbations}

The functionals $\Upsilon_{j}^{(a)}$ that appear in eq. (4.62) are

$$
\begin{aligned}
\Upsilon_{(1,1)}^{(2 t t)}(u)= & \frac{L^{2}}{4 f(u)^{2}}\left(\frac{1}{u^{2} e^{2 A(u)}}-\frac{f^{\prime}(u)^{2}}{f_{H}^{2} e^{2 A_{H}}}\right) \\
\Upsilon_{(1,1)}^{(2 z z)}(u)= & \frac{L^{2}}{4 u^{2} f(u) e^{2 A(u)}}(2-f(u)) \\
\Upsilon_{(1,1)}^{(2 t z)}(u)= & \frac{L^{2}}{4 u^{2} f(u) e^{2 A(u)}}, \\
\Upsilon_{2}^{(1 t)}(u)= & -\frac{L^{2}}{4 f(u)}\left\{\frac{1}{u^{2} f(u) e^{2 A(u)}}\right. \\
& \left.+\frac{f+2(1-u) f^{\prime}-\log \left(\frac{1-u}{f}\right)\left[\frac{f}{u}+4(1-u) A^{\prime} f+(1-u) f^{\prime}\right]}{(1-u)^{2} f_{H}^{2} e^{2 A_{H}}}\right\} .
\end{aligned}
$$

In all four cases the near-boundary expansion of the first integrand in eq. (4.64) reads

$$
w f(w) e^{4 A(w)} \Upsilon_{j}^{(a)}(w)= \pm \frac{A_{b} L^{2}}{4}\left(\frac{1}{w^{2}}-\frac{\phi_{L}^{2}}{12} \frac{1}{w}\right)+\mathcal{O}\left(w^{0}\right)
$$

where upper signs in this appendix refer to $a \in\{2 t t, 2 z z, 2 t z\}, j=(1,1)$, and lower signs refer to $a=1 t, j=2$. The first integral in eq. (4.64) thus admits the expansion

$$
\int_{1}^{v} \mathrm{~d} w w f(w) e^{4 A(w)} \Upsilon_{j}^{(a)}=\mp \frac{A_{b} L^{2}}{4}\left(\frac{1}{v}+\frac{\phi_{L}^{2}}{12} \log v\right)+c_{j}^{(a)}+\mathcal{O}(v)
$$


where the $v$-independent contribution $c_{j}^{(a)}$ can be extracted via

$$
c_{j}^{(a)}=\mp \frac{A_{b} L^{2}}{4}+\int_{1}^{0} \mathrm{~d} w\left[w f e^{4 A} \Upsilon_{j}^{(a)} \mp \frac{A_{b} L^{2}}{4 w^{2}}\left(1-\frac{\phi_{L}^{2}}{12} w\right)\right] .
$$

Plugging this into eq. (4.64), together with

$$
\frac{1}{v f(v) e^{4 A(v)}}=\frac{v}{A_{b}^{2}}\left(1+\frac{\phi_{L}^{2}}{6} v+\mathcal{O}\left(v^{2}\right)\right)
$$

one finally obtains the near-boundary expansion of the four $K_{j}^{(a)}$,

$$
K_{j}^{(a)}=\mp \frac{L^{2}}{4 A_{b}} u\left(1+\frac{\phi_{L}^{2}}{24} u \log u\right)+\frac{1}{2 A_{b}^{2}}\left(c_{j}^{(a)} \mp \frac{A_{b} L^{2} \phi_{L}^{2}}{32}\right) u^{2}+o\left(u^{2}\right),
$$

from which one can read off the sub-leading modes

$$
Y_{j}^{(a)}=\frac{1}{2 A_{b}^{2}}\left(c_{j}^{(a)} \mp \frac{A_{b} L^{2} \phi_{L}^{2}}{32}\right),
$$

yielding eq. (4.65) (recall expression (4.28) for the temperature).

\section{B.3 Leading backreaction of the scalar on AdS black branes}

This appendix describes the computation of the scalar's leading backreaction on the background metric used in subsection 4.6.1. The calculation goes along the lines presented in ref. [298]. Just this once we will be keeping the operator dimension $\Delta$ general, $2<\Delta<4$, as this can be done without difficulty and the general form of the results might be useful in other contexts. We thus consider potentials of the form

$$
V(\phi)=-\frac{12}{L^{2}}+\frac{\Delta(\Delta-4)}{2 L^{2}} \phi^{2}+\mathcal{O}\left(\phi^{3}\right) .
$$

At zeroth order, $\phi=0$, the background equations of motion (4.29) are solved by the $A d S_{5}$ black-brane metric (4.31), dual to the UV CFT. To first order in $\phi$, the regular solution to the scalar equation of motion, eq. (4.29a), linearised around the black-brane background is given by

$$
\begin{aligned}
\phi(u)=\delta \phi(u) \equiv & \phi_{H}{ }_{2} F_{1}\left(1-\Delta / 4, \Delta / 4 ; 1 ; 1-1 / u^{2}\right) \\
= & \delta \phi_{L} u^{(4-\Delta) / 2}{ }_{2} F_{1}\left(1-\Delta / 4,1-\Delta / 4 ; 2-\Delta / 2 ; u^{2}\right) \\
& +\delta \phi_{S L} u^{\Delta / 2}{ }_{2} F_{1}\left(\Delta / 4, \Delta / 4 ; \Delta / 2 ; u^{2}\right)
\end{aligned}
$$


with near-boundary modes

$$
\delta \phi_{L}=\phi_{H} \frac{\Gamma(\Delta / 2-1)}{\Gamma(\Delta / 4)^{2}}, \quad \delta \phi_{S L}=\phi_{H} \frac{\tan (\pi \Delta / 4)}{2 \pi} \frac{\Gamma(\Delta / 4)^{2}}{\Gamma(\Delta / 2)} .
$$

At second order, the scalar itself remains unchanged, but it backreacts on the background metric. Generally, the scalar backreacts on the geometry at even orders while $\phi$ itself receives corrections to its linearised solution (B.12) at odd orders. There is a minor complication concerning the boundary conditions: full non-perturbative solutions to the background equations of motion (4.29) depend on the single integration constant $\phi_{H}$ which parameterises the single physical parameter $T / \Lambda$. In the perturbative solution, on the other hand, we need to pick the value of the scalar at the horizon at each order in the perturbative series. This apparent ambiguity is resolved by the requirement that a chosen physical observable remain unchanged order by order. We simply choose to hold $\phi_{H}$ fixed, meaning that sub-leading corrections to $\phi(u)$ need to vanish at the horizon. Other possible, albeit more complicated, choices include fixing $A_{H}$ or $T / \Lambda$.

A related subtlety is constituted by the fact that $A(u)$ only enters the equations of motion (4.29) via its derivative $A^{\prime}(u)$. This is most conveniently dealt with by separating from the full non-perturbative $A(u)=\frac{1}{2} \log \left(A_{b} / u\right)+\mathcal{O}(u)$ the part $\tilde{A}(u)$ which vanishes at the boundary $u=0$, i.e. we write

$$
\begin{aligned}
A(u) & =\frac{1}{2} \log \left(\frac{A_{b}}{u}\right)+\tilde{A}(u) \\
& =\frac{1}{2} \log \left(\frac{A_{b}}{u}\right)+\int_{0}^{u} \mathrm{~d} v \tilde{A}^{\prime}(v) .
\end{aligned}
$$

The constant $A_{b}$ is fixed by the full global solution of the scalar as follows. In terms of the dimensionful $\zeta$-coordinate, eq. (B.35), close to the $A d S_{5}$ boundary we have $\phi \sim(\Lambda \zeta)^{4-\Delta}$ and $A \sim \log (L / \zeta)$, hence

$$
A \sim \log \left(\Lambda L \phi^{-1 /(4-\Delta)}\right)
$$

or, changing back to the $u$-coordinate,

$$
A=\frac{1}{2} \log \left(\frac{\left(\Lambda L \phi_{L}^{-1 /(4-\Delta)}\right)^{2}}{u}\right)+\mathcal{O}(u)
$$


and hence

$$
A_{b}=\left(\Lambda L \phi_{L}^{-1 /(4-\Delta)}\right)^{2}
$$

The value of $A(u)$, eq. (B.14), at the horizon and the Hawking temperature, eq. (4.28), are therefore given by

$$
A_{H}=\log \left(\Lambda L \phi_{L}^{-1 /(4-\Delta)}\right)+I, \quad T=\Lambda\left(\frac{f_{H} e^{I}}{2 \pi \phi_{L}^{1 /(4-\Delta)}}\right)
$$

where we defined

$$
I \equiv \int_{0}^{1} \mathrm{~d} u \tilde{A}^{\prime}(u)
$$

We emphasise again that these relations fix the observables $A_{H}$ and $T / \Lambda$ in terms of the full solutions for $\phi_{L}$ and $\tilde{A}(u)$. In the particular case of a perturbative solution, they determine $A_{H}$ and $T / \Lambda$ order by order in the expansion parameter $\phi_{H}$.

Let us now compute the leading, quadratic backreaction of the scalar on the geometry, which takes the form

$$
\tilde{A}(u)=\delta A(u), \quad f(u)=1-u^{2}+\delta f(u) .
$$

The corrections $\delta A$ and $\delta f$ are both of order $\mathcal{O}\left(\phi_{H}^{2}\right)$ and, from eqs. (4.29b) and $(4.29 \mathrm{~d})$, satisfy the two independent equations

$$
\begin{aligned}
\delta A^{\prime \prime}+\frac{1}{u} \delta A^{\prime} & =-\frac{1}{6}\left(\delta \phi^{\prime}\right)^{2} \\
\delta f^{\prime}-\frac{2}{u} \delta f & =-4\left(2-u^{2}\right) \delta A^{\prime}-\frac{\Delta(4-\Delta)}{12 u}(\delta \phi)^{2}-\frac{u\left(1-u^{2}\right)}{3}\left(\delta \phi^{\prime}\right)^{2} .
\end{aligned}
$$

The second-order equation (4.29c) for $f$ is redundant as it follows from the remaining three equations $(4.29 \mathrm{a}),(4.29 \mathrm{~b})$ and $(4.29 \mathrm{~d})$. Demanding that $\delta A$ vanish at the boundary $u=0$ we can integrate eq. (B.21a) twice and obtain

$$
\begin{aligned}
\delta A(u) & =-\int_{0}^{u} \mathrm{~d} v \frac{1}{v} \int_{0}^{v} \mathrm{~d} w w \frac{1}{6}\left(\delta \phi^{\prime}(v)\right)^{2} \\
& \stackrel{u \rightarrow 0}{\longrightarrow}-\frac{\phi_{L}^{2}}{24} u^{4-\Delta},
\end{aligned}
$$


in accordance with eq. (4.46a). Requiring that $\delta f$ vanish at the horizon $u=1$ we can now integrate eq. (B.21b) to get

$$
\begin{array}{r}
\delta f(u)=-u^{2} \int_{1}^{u} \mathrm{~d} v \frac{1}{v^{2}}\left[4\left(2-v^{2}\right) \delta A^{\prime}(v)\right. \\
+\frac{\Delta(4-\Delta)}{12 v}(\delta \phi(v))^{2} \\
\left.+\frac{v\left(1-v^{2}\right)}{3}\left(\delta \phi^{\prime}(v)\right)^{2}\right]
\end{array}
$$

The corresponding change in $f_{H}=-f^{\prime}(u=1)$ can be read off from eq. (B.21b):

$$
\begin{aligned}
& \delta f_{H}=\frac{\Delta(4-\Delta)}{12} \phi_{H}^{2}+4 \delta A^{\prime}(u=1) \\
& =\frac{\Delta(4-\Delta)}{12} \phi_{H}^{2}\left(1-\frac{\Delta(4-\Delta)}{8} \int_{0}^{1} \mathrm{~d} v v^{-5}\left[{ }_{2} F_{1}\left(2-\Delta / 4,1+\Delta / 4 ; 2 ; 1-1 / v^{2}\right)\right]^{2}\right) .
\end{aligned}
$$

Defining $\delta I \equiv \int_{0}^{1} \mathrm{~d} u \delta A^{\prime}(u)$, we obtain the following expressions for temperature $T$ and entropy density $s$ from (B.18):

$$
\begin{aligned}
4 G_{N} s & =e^{3 A_{H}}=\frac{(\Lambda L)^{3}}{\phi_{L}^{3 /(4-\Delta)}}\left(1+3 \delta I+\mathcal{O}\left(\phi_{H}^{4}\right)\right) \\
T / \Lambda & =\frac{\left(2+\delta f_{H}\right)(1+\delta I)+\mathcal{O}\left(\phi_{H}^{4}\right)}{2 \pi \phi_{L}^{1 /(4-\Delta)}}
\end{aligned}
$$

Note that sub-leading corrections to the scalar,

$$
\phi_{L}=\delta \phi_{L}+\mathcal{O}\left(\phi_{H}^{3}\right)=\frac{\Gamma(\Delta / 2-1)}{\Gamma(\Delta / 4)^{2}} \phi_{H}\left(1+\mathcal{O}\left(\phi_{H}^{2}\right)\right)
$$

enter expressions (B.25) at the same order as $\delta f_{H}$ and $\delta I$ do, so

$$
\left(\frac{\Lambda}{\pi T}\right)^{4-\Delta}=\frac{\Gamma(\Delta / 2-1)}{\Gamma(\Delta / 4)^{2}} \phi_{H}\left(1+\mathcal{O}\left(\phi_{H}^{2}\right)\right) .
$$

The sub-leading corrections to $\phi_{L}$ cancel, however, in the expression for the speed of sound, which becomes ${ }^{1}$

$$
c_{s}^{2}=\frac{\mathrm{d} \bar{p}}{\mathrm{~d} \bar{\epsilon}}=\frac{\mathrm{d} \log T}{\mathrm{~d} \log s}=\frac{1}{3}\left[1-(4-\Delta) \delta f_{H}\right]+\mathcal{O}\left(\phi_{H}^{4}\right) .
$$

${ }^{1}$ The result for $c_{s}^{2}$ agrees with the one computed in ref. [298] using a different radial coordinate. Note that the leading correction to the conformal value $1 / 3$ is negative for all $\Delta, 2<\Delta<4$. 


\section{B.4 Numerical construction of RG-flow geometries}

In this appendix we outline our construction of numerical background solutions to the action (5.13) for the scalar potentials $V_{(1)}$ and $V_{(2)}$ discussed in section 4.6. For that purpose it is convenient to use the scalar $\phi$ as radial coordinate [142], which is assumed to increase monotonically from the boundary $\phi=0$ to the horizon $\phi=\phi_{H}>0$. Defining

$$
e^{B(\phi)} \equiv \frac{L}{2 u} \frac{\mathrm{d} u}{\mathrm{~d} \phi}
$$

the background metric (4.27) takes the form

$$
\mathrm{d} s^{2}=g_{m n}^{(0)} \mathrm{d} x^{m} \mathrm{~d} x^{n}=e^{2 A(\phi)}\left[-f(\phi) \mathrm{d} t^{2}+\mathrm{d} \underline{x}^{2}\right]+\frac{e^{2 B(\phi)}}{f(\phi)} \mathrm{d} \phi^{2} .
$$

The residual scaling symmetry, inherited from the UV CFT, can be used to fix the value of the scalar source to $\Lambda=1 / L$ as the temperature is varied, knowing that all observables can only depend on the dimensionless ratio $T / \Lambda$.

The equations of motion (4.29) become

$$
\begin{aligned}
& 4 \frac{\mathrm{d} A}{\mathrm{~d} \phi}-\frac{\mathrm{d} B}{\mathrm{~d} \phi}+\frac{1}{f}\left(\frac{\mathrm{d} f}{\mathrm{~d} \phi}\right)-\frac{e^{2 B}}{f}\left(\frac{\mathrm{d} V}{\mathrm{~d} \phi}\right)=0, \\
& \frac{\mathrm{d}^{2} A}{\mathrm{~d} \phi^{2}}-\left(\frac{\mathrm{d} A}{\mathrm{~d} \phi}\right)\left(\frac{\mathrm{d} B}{\mathrm{~d} \phi}\right)+\frac{1}{6}=0, \\
& \frac{\mathrm{d}^{2} f}{\mathrm{~d} \phi^{2}}+\left[4 \frac{\mathrm{d} A}{\mathrm{~d} \phi}-\frac{\mathrm{d} B}{\mathrm{~d} \phi}\right] \frac{\mathrm{d} f}{\mathrm{~d} \phi}=0, \\
& 6\left(\frac{\mathrm{d} A}{\mathrm{~d} \phi}\right)\left(\frac{\mathrm{d} f}{\mathrm{~d} \phi}\right)+f\left[24\left(\frac{\mathrm{d} A}{\mathrm{~d} \phi}\right)^{2}-1\right]+2 e^{2 B} V=0,
\end{aligned}
$$

composed of a first-order equation for $B$ (B.31a), two second-order equations (B.31b)(B.31c) for $A$ and $f$, and the first-order constraint (B.31d). The system is partly redundant in the sense that the constraint (B.31d) and its derivative are algebraically given in terms of the other three equations:

$$
\begin{aligned}
\left(\frac{\mathrm{d}}{\mathrm{d} \phi}-2 \frac{\mathrm{d} B}{\mathrm{~d} \phi}\right)(\text { B.31d })=-2 f(\text { B.31a }) & +\left(48 f \frac{\mathrm{d} A}{\mathrm{~d} \phi}+6 \frac{\mathrm{d} f}{\mathrm{~d} \phi}\right) \\
& +6 \frac{\mathrm{d} A}{\mathrm{~d} \phi}(\text { B.31c })
\end{aligned}
$$


This redundancy prevents the constraint from restricting the series coefficients in the local near-horizon and near-boundary solutions which thus each involve five integration constants.

Imposing regularity on $A$ and $B$, the near-horizon expansion depends on three modes $\left\{A_{H}, f_{H}^{\phi}, \phi_{H}\right\}$ and reads

$$
\begin{aligned}
A(\phi) & =A_{H}-\frac{1}{3} \frac{V\left(\phi_{H}\right)}{V^{\prime}\left(\phi_{H}\right)}\left(\phi-\phi_{H}\right)+\sum_{k \geq 2} b_{k}^{A, \phi}\left(\phi-\phi_{H}\right)^{k}, \\
f(\phi) & =\left(\phi-\phi_{H}\right)\left[f_{H}^{\phi}+\sum_{k \geq 1} b_{k}^{f, \phi}\left(\phi-\phi_{H}\right)^{k}\right], \\
B(\phi) & =\frac{1}{2} \log \left(\frac{f_{H}^{\phi}}{V^{\prime}\left(\phi_{H}\right)}\right)+\sum_{k \geq 1} b_{k}^{B}\left(\phi-\phi_{H}\right)^{k}
\end{aligned}
$$

with all series coefficients fixed in terms of the near-horizon modes and the chosen potential $V(\phi)$. Inserting the near-horizon expansions of $\phi(u)$, eq. (4.44c), into eqs. (B.33) and equating the result with the near-horizon solution of $A(u)$ and $B(u)$, eqs. (4.44), relates $f_{H}^{\phi}$ to the near-horizon mode $f_{H}$ in the $u$-coordinate, ensuring that near-horizon solutions satisfy eq. (B.29). In particular, it follows from eq. (B.29) and $\phi(u)$ 's nearhorizon expansion, eq. (4.44c), that

$$
f_{H}=-\frac{L^{2} V^{\prime}\left(\phi_{H}\right)}{2} \frac{e^{B\left(\phi_{H}\right)}}{L} .
$$

In order to determine which boundary conditions we must impose on the fields for the spacetime to be asymptotically $A d S_{5}$, let us switch to a radial coordinate $\zeta$ in terms of which the line element (4.27) reads

$$
\mathrm{d} s^{2}=g_{m n}^{(0)} \mathrm{d} x^{m} \mathrm{~d} x^{n}=e^{2 A}\left[-f \mathrm{~d} t^{2}+\mathrm{d} \underline{x}^{2}\right]+\frac{L^{2}}{\zeta^{2} f} \mathrm{~d} \zeta^{2}
$$

For this metric to approach $A d S_{5}$ as $\zeta \rightarrow 0$, eq. (4.26), $A$ and $f$ need to behave as

$$
A \sim \log \left(\frac{L}{\zeta}\right), \quad f \sim 1 .
$$

Combining this with the leading near-boundary behaviour of the scalar, $\phi \sim \Lambda \zeta$, one finds that

$$
A=-\log \phi+\log (\Lambda L)+o(1), \quad f=1+o(1)
$$


Imposing these boundary conditions and setting the sub-leading mode $\log (\Lambda L)$ of $A$ to zero by scaling the operator source $\Lambda$ to $\Lambda=1 / L$, near-boundary solutions to (B.31) assume the form

$$
A(\phi)=-\log \phi+\mathcal{O}\left(\phi^{2}\right), \quad f(\phi)=1+\mathcal{O}\left(\phi^{4}\right), \quad B(\phi)=\log \left(\frac{L}{\phi}\right)+\mathcal{O}\left(\phi^{2}\right)
$$

Matching eq. (B.37) with the near-boundary expansions of $A(u)$ and $\phi(u)$, eq. (4.46a), provides the relation ${ }^{2}$

$$
A_{b}=\left(\frac{\Lambda L}{\phi_{L}}\right)^{2}=\frac{1}{\phi_{L}^{2}}
$$

To construct global solutions that connect the local solutions (B.33) and (B.38), we employed the method developed in ref. [142]. The key step is the realisation that a decoupled non-linear second-order equation for $G(\phi) \equiv A^{\prime}(\phi)$ can be derived from eqs. (B.31): ${ }^{3}$

$$
\frac{G^{\prime}(\phi)}{G+V /\left(3 V^{\prime}(\phi)\right)}=\frac{\mathrm{d}}{\mathrm{d} \phi} \log \left(\frac{G^{\prime}(\phi)}{G(\phi)}+\frac{1}{6 G(\phi)}-4 G(\phi)-\frac{G^{\prime}(\phi)}{G+V /\left(3 V^{\prime}(\phi)\right)}\right) .
$$

Imposing regularity at the horizon, eq. (B.33a), global solutions to eq. (B.40) depend on the single integration constant $\phi_{H}$ and can readily be produced numerically. Solutions for $A(\phi), B(\phi)$ and $f(\phi)$ are then obtained by simple integrations of the equations of motion (B.31). They depend on four additional integration constants which are fixed by requiring that the spacetime be asymptotically $A d S_{5}$, eq. (B.38), and that $f(\phi)$ vanish at the horizon:

$$
\begin{aligned}
& A(\phi)=-\log \phi+\int_{0}^{\phi} \mathrm{d} \varphi\left(G(\varphi)+\frac{1}{\varphi}\right) \\
& B(\phi)=\log \left(\frac{L}{\phi}\right)+\int_{0}^{\phi} \mathrm{d} \varphi\left(\frac{G^{\prime}(\varphi)+1 / 6}{G(\varphi)}+\frac{1}{\varphi}\right) \\
& f(\phi)=\frac{\int_{\phi}^{\phi_{H}} \mathrm{~d} \varphi \exp [-4 A(\varphi)+B(\varphi)]}{\int_{0}^{\phi_{H}} \mathrm{~d} \varphi \exp [-4 A(\varphi)+B(\varphi)]}
\end{aligned}
$$

\footnotetext{
${ }^{2}$ Compare with eq. (B.17).

${ }^{3}$ This possibility is hinted at by the observation that $A(\phi)$ 's near-horizon expansion turns out to be independent of the mode $f_{H}^{\phi}$, and by the fact that $A(\phi)$ enters eqs. (B.31) only through its derivative.
} 
These solutions depend on the single constant $\phi_{H}$ which parameterises the single physical parameter $T / \Lambda=T L$.

Ultimately, we are looking for global solutions $A(u), f(u), \phi(u)$ in terms of the $u$ coordinate, which we found to be a lot more convenient when dealing with perturbations of the metric. For this purpose, we first determine the expansion of a global solution (B.41) near the horizon $u=1$ by computing the modes $A_{H}=A\left(\phi_{H}\right)$ and $f_{H}$ from relation (B.34), and plugging the result into the near-horizon expansions (4.44) of $A(u)$, $f(u)$, and $\phi(u)$. We obtain a global solution without further numerical integrations by matching this near-horizon expansion directly with the near-boundary expansion (4.46) at an intermediate value of $u$ where both local solutions are valid. The fact that the near-horizon modes stem from a global solution to the connection problem ensures that these solutions indeed display the appropriate near-boundary behaviour, which can be verified by checking relation (B.39).

For the computation of $f_{H}$ it is helpful to use

$$
G\left(\phi_{H}\right)=-\frac{1}{3} \frac{V\left(\phi_{H}\right)}{V^{\prime}\left(\phi_{H}\right)}, \quad \phi G(\phi)=\phi A^{\prime}(\phi) \stackrel{\phi \rightarrow 0}{\longrightarrow}-1,
$$

which follow from eqs. (B.33a) and (B.38), in order to re-write expression (B.34):

$$
\begin{aligned}
f_{H} & =-\frac{L^{2} V^{\prime}\left(\phi_{H}\right)}{2} \frac{e^{B\left(\phi_{H}\right)}}{L} \\
& =\left(\frac{L^{2} V\left(\phi_{H}\right)}{6 G\left(\phi_{H}\right)}\right) \frac{1}{\phi_{H}} \exp \left\{\lim \left[\log \left(\frac{-G\left(\phi_{H}\right)}{-G(\phi)}\right)+\log \left(\frac{\phi_{H}}{\phi}\right)\right]+\int_{0}^{\phi_{H}} \mathrm{~d} \varphi \frac{1}{6 G(\varphi)}\right\} \\
& =-\frac{L^{2} V\left(\phi_{H}\right)}{6} \exp \left\{\int_{0}^{\phi_{H}} \mathrm{~d} \varphi \frac{1}{6 G(\varphi)}\right\} .
\end{aligned}
$$

Hawking temperature $T$ and entropy density $s$, eq. (4.28), are then given by

$$
\begin{gathered}
T L=\frac{f_{H} e^{A_{H}}}{2 \pi}=-\frac{L^{2} V\left(\phi_{H}\right)}{12 \pi} \frac{1}{\phi_{H}} \exp \left\{\int_{0}^{\phi_{H}} \mathrm{~d} \varphi\left(G(\varphi)+\frac{1}{\varphi}+\frac{1}{6 G(\varphi)}\right)\right\} \\
4 G_{N} s=e^{3 A_{H}}=\frac{1}{\phi_{H}^{3}} \exp \left\{3 \int_{0}^{\phi_{H}} \mathrm{~d} \varphi\left(G\left(\phi_{H}\right)+\frac{1}{\varphi}\right)\right\}
\end{gathered}
$$


The leading high-temperature asymptotics of (B.44) are obtained by taking the limit $\phi_{H} \rightarrow 0$, recalling that $V(0)=-12 / L^{2}$,

$$
\begin{gathered}
T L \stackrel{\phi_{H} \rightarrow 0}{\longrightarrow} \frac{1}{\pi \phi_{H}} \exp \left\{\lim _{\phi_{H} \rightarrow 0}\left(\int_{0}^{\phi_{H}} \mathrm{~d} \varphi G(\varphi)\right)\right\}, \\
4 G_{N} s \stackrel{\phi_{H} \rightarrow 0}{\longrightarrow} \frac{1}{\phi_{H}^{3}} \exp \left\{3 \lim _{\phi_{H} \rightarrow 0}\left(\int_{0}^{\phi_{H}} \mathrm{~d} \varphi G(\varphi)\right)\right\} .
\end{gathered}
$$

The limit of the remaining integral does not vanish, by virtue of the fact that $G(\phi)$, whose equation of motion (B.40) has regular singular points at $\phi=0$ and $\phi=\phi_{H}$, does not behave smoothly in the limit $\phi_{H} \rightarrow 0 .{ }^{4}$ However, the limiting value can be computed by comparison with the perturbative high-T solution, eqs. (B.13) and (B.25):

$$
T / \Lambda=T L \stackrel{\phi_{H} \rightarrow 0}{\longrightarrow} \frac{1}{\pi \phi_{H}} \frac{\Gamma(3 / 4)^{2}}{\sqrt{\pi}}, \quad 4 G_{N} s \stackrel{\phi_{H} \rightarrow 0}{\longrightarrow}\left(\frac{\Gamma(3 / 4)^{2}}{\phi_{H} \sqrt{\pi}}\right)^{3} .
$$

Details on the numerics For integrals (B.41)-(B.44) in the $\phi$-coordinate, a nearhorizon expansion of $G(\phi)$ to eleventh order was used in the region $\phi_{H}-\phi<10^{-2}$. We verified that the dependence of $A_{H}$ and $f_{H}$ on $\phi_{H}^{4}$, which is sub-leading compared to the $\phi_{H^{-}}^{2}$-contribution from appendix B.3 but completely dictated by the quartic term (4.47) common to all potentials, is the same for all solutions. The local solutions (4.44) and (4.46) in the $u$-coordinate were expanded to sixteen orders beyond horizon and boundary modes. Inverting the near-boundary expansion, we extracted the boundary modes $A_{b}, f_{b}, \phi_{L}, \phi_{S L}$ by matching the two local solutions at $u=0.5$. The numerical error due to the truncation of the two series is of order $0.5^{17} \sim 8 \cdot 10^{-6}$. We checked that relations (4.68) and (B.39) between horizon and boundary modes were indeed satisfied with a numerical error smaller than $10^{-5}$ by all considered solutions. Directly matching the two local solutions in the $u$-coordinate, rather than numerically integrating from the horizon towards the boundary, involved the somewhat tedious inversion of the nearboundary expansion to sixteen orders. However, it greatly simplified the computation of the transport coefficients because the integrals (4.65) over the global background solution split into two simple integrals over the near-horizon and the near-boundary series solutions.

\footnotetext{
${ }^{4}$ We believe that this point was overlooked in ref. [142].
} 


\section{Bibliography}

[1] J. M. Maldacena, The Large N limit of superconformal field theories and supergravity, Int. J. Theor. Phys. 38 (1999) 1113-1133, [hep-th/9711200].

[2] S. S. Gubser, I. R. Klebanov and A. M. Polyakov, Gauge theory correlators from noncritical string theory, Phys. Lett. B428 (1998) 105-114, [hep-th/9802109].

[3] E. Witten, Anti-de Sitter space and holography, Adv. Theor. Math. Phys. 2 (1998) 253-291, [hep-th/9802150].

[4] O. Aharony, S. S. Gubser, J. M. Maldacena, H. Ooguri and Y. Oz, Large $N$ field theories, string theory and gravity, Phys. Rept. 323 (2000) 183-386, [hep-th/9905111].

[5] G. 't Hooft, Dimensional reduction in quantum gravity, in Salamfest 1993:0284-296, pp. 0284-296, 1993. gr-qc/9310026.

[6] L. Susskind, The World as a hologram, J. Math. Phys. 36 (1995) 6377-6396, [hep-th/9409089].

[7] P. Kovtun, D. T. Son and A. O. Starinets, Viscosity in strongly interacting quantum field theories from black hole physics, Phys. Rev. Lett. 94 (2005) 111601, [hep-th/0405231].

[8] J. Casalderrey-Solana, H. Liu, D. Mateos, K. Rajagopal and U. A. Wiedemann, Gauge/String Duality, Hot QCD and Heavy Ion Collisions. Cambridge University Press, 2014, 10.1017/CBO9781139136747.

[9] S. A. Hartnoll, A. Lucas and S. Sachdev, Holographic quantum matter, 1612.07324.

[10] R. Baier, P. Romatschke, D. T. Son, A. O. Starinets and M. A. Stephanov, Relativistic viscous hydrodynamics, conformal invariance, and holography, JHEP 04 (2008) 100, [0712.2451].

[11] S. Bhattacharyya, V. E. Hubeny, S. Minwalla and M. Rangamani, Nonlinear Fluid Dynamics from Gravity, JHEP 02 (2008) 045, [0712.2456].

[12] S. S. Gubser, Breaking an Abelian gauge symmetry near a black hole horizon, Phys. Rev. D78 (2008) 065034, [0801.2977].

[13] P. Kleinert and J. Probst, Second-Order Hydrodynamics and Universality in Non-Conformal Holographic Fluids, JHEP 12 (2016) 091, [1610.01081].

[14] M. Haack and A. Yarom, Universality of second order transport coefficients from the gauge-string duality, Nucl. Phys. B813 (2009) 140-155, [0811.1794].

[15] A. O'Bannon, I. Papadimitriou and J. Probst, A Holographic Two-Impurity Kondo Model, JHEP 01 (2016) 103, [1510.08123]. 
[16] J. Erdmenger, C. Hoyos, A. O'Bannon, I. Papadimitriou, J. Probst and J. M. S. Wu, Holographic Kondo and Fano Resonances, Phys. Rev. D96 (2017) 021901, [1611.09368].

[17] J. Erdmenger, C. Hoyos, A. O'Bannon, I. Papadimitriou, J. Probst and J. M. S. Wu, Two-point Functions in a Holographic Kondo Model, JHEP 03 (2017) 039, [1612.02005].

[18] J. Erdmenger, C. Hoyos, A. O'Bannon and J. Wu, A Holographic Model of the Kondo Effect, JHEP 12 (2013) 086, [1310.3271].

[19] M. B. Green, J. H. Schwarz and E. Witten, Superstring Theory. Vol. 1: Introduction. Cambridge University Press, 1988.

[20] M. B. Green, J. H. Schwarz and E. Witten, Superstring Theory. Vol. 2: Loop Amplitudes, Anomalies and Phenomenology. Cambridge University Press, 1988.

[21] J. Polchinski, String Theory. Vol. 1: An Introduction to the Bosonic String. Cambridge University Press, 2007.

[22] J. Polchinski, String Theory. Vol. 2: Superstring Theory and Beyond. Cambridge University Press, 2007.

[23] D. Tong, String Theory, 0908.0333.

[24] C. V. Johnson, D-Branes. Cambridge University Press, 2003.

[25] L. Thorlacius, Introduction to D-branes, Nucl. Phys. Proc. Suppl. 61A (1998) 86-98, [hep-th/9708078].

[26] E. S. Fradkin and A. A. Tseytlin, Effective Field Theory from Quantized Strings, Phys. Lett. B158 (1985) 316-322.

[27] E. S. Fradkin and A. A. Tseytlin, Nonlinear Electrodynamics from Quantized Strings, Phys. Lett. B163 (1985) 123-130.

[28] E. Witten, Bound states of strings and p-branes, Nucl. Phys. B460 (1996) 335-350, [hep-th/9510135].

[29] J. Dai, R. G. Leigh and J. Polchinski, New Connections Between String Theories, Mod. Phys. Lett. A4 (1989) 2073-2083.

[30] J. Polchinski, Dirichlet Branes and Ramond-Ramond charges, Phys. Rev. Lett. 75 (1995) 4724-4727, [hep-th/9510017].

[31] J. Polchinski, Introduction to Gauge/Gravity Duality, in Proceedings, Theoretical Advanced Study Institute in Elementary Particle Physics (TASI 2010). String Theory and Its Applications: From meV to the Planck Scale: Boulder, Colorado, USA, June 1-25, 2010, pp. 3-46, 2010. 1010.6134. DOI.

[32] S. S. Gubser and A. Hashimoto, Exact absorption probabilities for the D3-brane, Commun. Math. Phys. 203 (1999) 325-340, [hep-th/9805140].

[33] R. C. Myers, A. O. Starinets and R. M. Thomson, Holographic spectral functions and diffusion constants for fundamental matter, JHEP 11 (2007) 091, [0706.0162]. 
[34] G. 't Hooft, A Planar Diagram Theory for Strong Interactions, Nucl. Phys. B72 (1974) 461.

[35] S. Coleman, Aspects of Symmetry: Selected Erice Lectures. Cambridge University Press, 1985, 10.1017/CBO9780511565045.

[36] S. S. Gubser, I. R. Klebanov and A. A. Tseytlin, Coupling constant dependence in the thermodynamics of $N=4$ supersymmetric Yang-Mills theory, Nucl. Phys. B534 (1998) 202-222, [hep-th/9805156].

[37] J. Pawelczyk and S. Theisen, AdS(5) $x S^{* * 5}$ black hole metric at O(alpha-prime**3), JHEP 09 (1998) 010, [hep-th/9808126].

[38] M. Ammon and J. Erdmenger, Gauge/Gravity Duality. Cambridge University Press, 2015.

[39] J. Erlich, E. Katz, D. T. Son and M. A. Stephanov, QCD and a holographic model of hadrons, Phys. Rev. Lett. 95 (2005) 261602, [hep-ph/0501128].

[40] A. Karch, E. Katz, D. T. Son and M. A. Stephanov, Linear confinement and AdS/QCD, Phys. Rev. D74 (2006) 015005, [hep-ph/0602229].

[41] H. Liu, J. McGreevy and D. Vegh, Non-Fermi liquids from holography, Phys. Rev. D83 (2011) 065029, [0903.2477].

[42] T. Faulkner, H. Liu, J. McGreevy and D. Vegh, Emergent quantum criticality, Fermi surfaces, and AdS(2), Phys. Rev. D83 (2011) 125002, [0907.2694].

[43] G. Policastro, D. T. Son and A. O. Starinets, The Shear viscosity of strongly coupled $N=4$ supersymmetric Yang-Mills plasma, Phys. Rev. Lett. 87 (2001) 081601, [hep-th/0104066].

[44] S. R. Das, G. W. Gibbons and S. D. Mathur, Universality of low-energy absorption cross-sections for black holes, Phys. Rev. Lett. 78 (1997) 417-419, [hep-th/9609052].

[45] I. R. Klebanov, World volume approach to absorption by nondilatonic branes, Nucl. Phys. B496 (1997) 231-242, [hep-th/9702076].

[46] S. S. Gubser, I. R. Klebanov and A. A. Tseytlin, String theory and classical absorption by three-branes, Nucl. Phys. B499 (1997) 217-240, [hep-th/9703040].

[47] D. T. Son and A. O. Starinets, Minkowski space correlators in AdS / CFT correspondence: Recipe and applications, JHEP 09 (2002) 042, [hep-th/0205051].

[48] J. L. Petersen, Introduction to the Maldacena conjecture on AdS / CFT, Int. J. Mod. Phys. A14 (1999) 3597-3672, [hep-th/9902131].

[49] M. Gunaydin and N. Marcus, The spectrum of the $S^{5}$ compactification of the chiral $N=2, D=10$ supergravity and the unitary supermultiplets of U(2,2/4), Classical and Quantum Gravity 2 (1985) L11.

[50] H. J. Kim, L. J. Romans and P. van Nieuwenhuizen, Mass spectrum of chiral ten-dimensional $N=2$ supergravity on $S^{5}$, Phys. Rev. D 32 (Jul, 1985) 389-399.

[51] C. Bender and S. Orszag, Advanced Mathematical Methods for Scientists and Engineers I. Springer-Verlag, New York, 1999. 
[52] V. Balasubramanian, P. Kraus and A. E. Lawrence, Bulk versus boundary dynamics in anti-de Sitter space-time, Phys. Rev. D59 (1999) 046003, [hep-th/9805171].

[53] S. de Haro, S. N. Solodukhin and K. Skenderis, Holographic reconstruction of space-time and renormalization in the AdS / CFT correspondence, Commun. Math. Phys. 217 (2001) 595-622, [hep-th/0002230].

[54] K. Skenderis, Lecture notes on holographic renormalization, Class. Quant. Grav. 19 (2002) 5849-5876, [hep-th/0209067].

[55] P. Breitenlohner and D. Z. Freedman, Positive energy in anti-de Sitter backgrounds and gauged extended supergravity, Physics Letters B 115 (1982) 197 - 201.

[56] P. Breitenlohner and D. Z. Freedman, Stability in gauged extended supergravity, Annals of Physics 144 (1982) 249 - 281.

[57] S.-J. Rey and J.-T. Yee, Macroscopic strings as heavy quarks in large $N$ gauge theory and anti-de Sitter supergravity, Eur. Phys. J. C22 (2001) 379-394, [hep-th/9803001].

[58] L. Susskind and E. Witten, The Holographic bound in anti-de Sitter space, hep-th/9805114.

[59] A. M. Polyakov, The Wall of the cave, Int. J. Mod. Phys. A14 (1999) 645-658, [hep-th/9809057].

[60] M. Henningson and K. Skenderis, The Holographic Weyl anomaly, JHEP 07 (1998) 023, [hep-th/9806087].

[61] M. Henningson and K. Skenderis, Holography and the Weyl anomaly, Fortsch. Phys. 48 (2000) 125-128, [hep-th/9812032].

[62] V. Balasubramanian and P. Kraus, A Stress tensor for Anti-de Sitter gravity, Commun. Math. Phys. 208 (1999) 413-428, [hep-th/9902121].

[63] W. Mueck and K. S. Viswanathan, Counterterms for the Dirichlet prescription of the AdS / CFT correspondence, hep-th/9905046.

[64] J. de Boer, E. P. Verlinde and H. L. Verlinde, On the holographic renormalization group, JHEP 08 (2000) 003, [hep-th/9912012].

[65] J. de Boer, The Holographic Renormalization Group, Fortsch. Phys. 49 (2001) 339-358, [hep-th/0101026].

[66] I. Papadimitriou and K. Skenderis, AdS / CFT correspondence and geometry, IRMA Lect. Math. Theor. Phys. 8 (2005) 73-101, [hep-th/0404176].

[67] N. Iqbal and H. Liu, Universality of the hydrodynamic limit in AdS/CFT and the membrane paradigm, Phys. Rev. D79 (2009) 025023, [0809.3808].

[68] L. Girardello, M. Petrini, M. Porrati and A. Zaffaroni, Novel local CFT and exact results on perturbations of $N=4$ superYang Mills from AdS dynamics, JHEP 12 (1998) 022, [hep-th/9810126].

[69] D. Z. Freedman, S. S. Gubser, K. Pilch and N. P. Warner, Renormalization group flows from holography supersymmetry and a $c$ theorem, Adv. Theor. Math. Phys. 3 (1999) 363-417, [hep-th/9904017]. 
[70] E. Witten, Anti-de Sitter space, thermal phase transition, and confinement in gauge theories, Adv. Theor. Math. Phys. 2 (1998) 505-532, [hep-th/9803131].

[71] S. A. Hartnoll, Lectures on holographic methods for condensed matter physics, Class. Quant. Grav. 26 (2009) 224002, [0903.3246].

[72] I. R. Klebanov and E. Witten, AdS / CFT correspondence and symmetry breaking, Nucl. Phys. B556 (1999) 89-114, [hep-th/9905104].

[73] C. P. Herzog and D. T. Son, Schwinger-Keldysh propagators from AdS/CFT correspondence, JHEP 03 (2003) 046, [hep-th/0212072].

[74] P. K. Kovtun and A. O. Starinets, Quasinormal modes and holography, Phys. Rev. D72 (2005) 086009, [hep-th/0506184].

[75] D. Z. Freedman, S. D. Mathur, A. Matusis and L. Rastelli, Correlation functions in the CFT(d) / AdS (d+1) correspondence, Nucl. Phys. B546 (1999) 96-118, [hep-th/9804058].

[76] G. Chalmers, H. Nastase, K. Schalm and R. Siebelink, $R$ current correlators in $N=4$ superYang-Mills theory from anti-de Sitter supergravity, Nucl. Phys. B540 (1999) 247-270, [hep-th/9805105].

[77] J. M. Maldacena, Wilson loops in large N field theories, Phys. Rev. Lett. 80 (1998) 4859-4862, [hep-th/9803002].

[78] S.-J. Rey, S. Theisen and J.-T. Yee, Wilson-Polyakov loop at finite temperature in large N gauge theory and anti-de Sitter supergravity, Nucl. Phys. B527 (1998) 171-186, [hep-th/9803135].

[79] A. Karch and E. Katz, Adding flavor to AdS / CFT, JHEP 06 (2002) 043, [hep-th/0205236].

[80] I. R. Klebanov and M. J. Strassler, Supergravity and a confining gauge theory: Duality cascades and chi SB resolution of naked singularities, JHEP 08 (2000) 052, [hep-th/0007191].

[81] T. Sakai and S. Sugimoto, Low energy hadron physics in holographic QCD, Prog. Theor. Phys. 113 (2005) 843-882, [hep-th/0412141].

[82] T. Sakai and S. Sugimoto, More on a holographic dual of QCD, Prog. Theor. Phys. 114 (2005) 1083-1118, [hep-th/0507073].

[83] T. Nishioka, S. Ryu and T. Takayanagi, Holographic Entanglement Entropy: An Overview, J. Phys. A42 (2009) 504008, [0905.0932].

[84] S. Ryu and T. Takayanagi, Holographic derivation of entanglement entropy from AdS/CFT, Phys. Rev. Lett. 96 (2006) 181602, [hep-th/0603001].

[85] S. Ryu and T. Takayanagi, Aspects of Holographic Entanglement Entropy, JHEP 08 (2006) 045, [hep-th/0605073].

[86] A. O'Bannon, J. Probst, R. Rodgers and C. F. Uhlemann, First law of entanglement rates from holography, Phys. Rev. D96 (2017) 066028, [1612.07769].

[87] L. P. Kadanoff and P. C. Martin, Hydrodynamic equations and correlation functions, Annals of Physics 24 (Oct., 1963) 419-469. 
[88] M. S. Green, Markoff Random Processes and the Statistical Mechanics of Time-Dependent Phenomena. II. Irreversible Processes in Fluids, The Journal of Chemical Physics 22 (1954) 398-413, [http://dx.doi.org/10.1063/1.1740082].

[89] R. Kubo, Statistical-Mechanical Theory of Irreversible Processes. I. General Theory and Simple Applications to Magnetic and Conduction Problems, Journal of the Physical Society of Japan 12 (1957) 570-586, [http://dx.doi.org/10.1143/JPSJ.12.570].

[90] P. Kovtun, Lectures on hydrodynamic fluctuations in relativistic theories, J. Phys. A45 (2012) 473001, [1205.5040].

[91] L. D. Landau and E. M. Lifshitz, Fluid Mechanics. Butterworth-Heinemann, Oxford, second ed., 1987.

[92] W. Israel, Thermodynamics of relativistic systems, Physica A: Statistical Mechanics and its Applications 106 (1981) $204-214$.

[93] P. Romatschke, Relativistic Viscous Fluid Dynamics and Non-Equilibrium Entropy, Class. Quant. Grav. 27 (2010) 025006, [0906.4787].

[94] D. T. Son and A. O. Starinets, Viscosity, Black Holes, and Quantum Field Theory, Ann. Rev. Nucl. Part. Sci. 57 (2007) 95-118, [0704.0240].

[95] G. Policastro, D. T. Son and A. O. Starinets, From AdS / CFT correspondence to hydrodynamics, JHEP 09 (2002) 043, [hep-th/0205052].

[96] G. Policastro, D. T. Son and A. O. Starinets, From AdS / CFT correspondence to hydrodynamics. 2. Sound waves, JHEP 12 (2002) 054, [hep-th/0210220].

[97] G. D. Mahan, Many-Particle Physics. Plenum Press, New York, second ed., 1993.

[98] P. B. Allen, Electron Transport, in Conceptual Foundations of Material Properties: A standard model for calculation of ground-and excited-state properties (Cohen and Louie, eds.), pp. 165-218. Elsevier, Amsterdam, 2006.

[99] G. T. Horowitz, Introduction to Holographic Superconductors, Lect. Notes Phys. $\mathbf{8 2 8}$ (2011) 313-347, [1002.1722].

[100] F. London and H. London, The Electromagnetic Equations of the Supraconductor, Proceedings of the Royal Society of London A: Mathematical, Physical and Engineering Sciences 149 (1935) 71-88,

[http://rspa.royalsocietypublishing.org/content/149/866/71.full.pdf].

[101] J. F. Annett, Superconductivity, Superfluids, and Condensates. Oxford University Press, 2004.

[102] N. W. Ashcroft and N. D. Mermin, Solid State Physics. Harcourt, Orlando, FL, 1976.

[103] C. P. Herzog, Lectures on Holographic Superfluidity and Superconductivity, J. Phys. A42 (2009) 343001, [0904.1975].

[104] S. Sachdev, What can gauge-gravity duality teach us about condensed matter physics?, Ann. Rev. Condensed Matter Phys. 3 (2012) 9-33, [1108.1197].

[105] J. Zaanen, Y.-W. Sun, Y. Liu and K. Schalm, Holographic Duality in Condensed Matter Physics. Cambridge University Press, 2015. 
[106] S. A. Hartnoll, C. P. Herzog and G. T. Horowitz, Building a Holographic Superconductor, Phys. Rev. Lett. 101 (2008) 031601, [0803.3295].

[107] S. A. Hartnoll, C. P. Herzog and G. T. Horowitz, Holographic Superconductors, JHEP 12 (2008) 015, [0810.1563].

[108] A. Karch and A. O'Bannon, Metallic AdS/CFT, JHEP 09 (2007) 024, [0705.3870].

[109] D. Forster, Hydrodynamic Fluctuations, Broken Symmetry, and Correlation Functions. Addison-Wesley, Redwood City, CA, 1990.

[110] U. Fano, Effects of Configuration Interaction on Intensities and Phase Shifts, Phys. Rev. 124 (Dec, 1961) 1866-1878.

[111] A. E. Miroshnichenko, S. Flach and Y. S. Kivshar, Fano resonances in nanoscale structures, Rev. Mod. Phys. 82 (Aug, 2010) 2257-2298.

[112] Y. S. Joe, A. M. Satanin and C. S. Kim, Classical analogy of Fano resonances, Physica Scripta 74 (2006) 259.

[113] G. D. Moore and K. A. Sohrabi, Kubo Formulae for Second-Order Hydrodynamic Coefficients, Phys. Rev. Lett. 106 (2011) 122302, [1007.5333].

[114] G. D. Moore and K. A. Sohrabi, Thermodynamical second-order hydrodynamic coefficients, JHEP 11 (2012) 148, [1210.3340].

[115] M. Gyulassy and L. McLerran, New forms of QCD matter discovered at RHIC, Nucl. Phys. A750 (2005) 30-63, [nucl-th/0405013].

[116] BRAHMS collaboration, I. Arsene et al., Quark gluon plasma and color glass condensate at RHIC? The Perspective from the BRAHMS experiment, Nucl. Phys. A757 (2005) 1-27, [nucl-ex/0410020].

[117] B. B. Back et al., The PHOBOS perspective on discoveries at RHIC, Nucl. Phys. A757 (2005) 28-101, [nucl-ex/0410022].

[118] PHENIX collaboration, K. Adcox et al., Formation of dense partonic matter in relativistic nucleus-nucleus collisions at RHIC: Experimental evaluation by the PHENIX collaboration, Nucl. Phys. A757 (2005) 184-283, [nucl-ex/0410003].

[119] STAR collaboration, J. Adams et al., Experimental and theoretical challenges in the search for the quark gluon plasma: The STAR Collaboration's critical assessment of the evidence from RHIC collisions, Nucl. Phys. A757 (2005) 102-183, [nucl-ex/0501009].

[120] G. D. Moore and O. Saremi, Bulk viscosity and spectral functions in QCD, JHEP 09 (2008) 015, [0805.4201].

[121] P. Kovtun, D. T. Son and A. O. Starinets, Holography and hydrodynamics: Diffusion on stretched horizons, JHEP 10 (2003) 064, [hep-th/0309213].

[122] A. Buchel and J. T. Liu, Universality of the shear viscosity in supergravity, Phys. Rev. Lett. 93 (2004) 090602, [hep-th/0311175].

[123] A. Buchel, On universality of stress-energy tensor correlation functions in supergravity, Phys. Lett. B609 (2005) 392-401, [hep-th/0408095]. 
[124] A. O. Starinets, Quasinormal spectrum and the black hole membrane paradigm, Phys. Lett. B670 (2009) 442-445, [0806.3797].

[125] R. Brustein and A. J. M. Medved, The Ratio of shear viscosity to entropy density in generalized theories of gravity, Phys. Rev. D79 (2009) 021901, [0808.3498].

[126] S. Cremonini, The Shear Viscosity to Entropy Ratio: A Status Report, Mod. Phys. Lett. B25 (2011) 1867-1888, [1108.0677].

[127] D. Teaney, The Effects of viscosity on spectra, elliptic flow, and HBT radii, Phys. Rev. C68 (2003) 034913, [nucl-th/0301099].

[128] E. Shuryak, Why does the quark gluon plasma at RHIC behave as a nearly ideal fluid?, Prog. Part. Nucl. Phys. 53 (2004) 273-303, [hep-ph/0312227].

[129] P. Romatschke and U. Romatschke, Viscosity Information from Relativistic Nuclear Collisions: How Perfect is the Fluid Observed at RHIC?, Phys. Rev. Lett. 99 (2007) 172301, [0706.1522].

[130] H. Song and U. W. Heinz, Suppression of elliptic flow in a minimally viscous quark-gluon plasma, Phys. Lett. B658 (2008) 279-283, [0709.0742].

[131] K. Dusling and D. Teaney, Simulating elliptic flow with viscous hydrodynamics, Phys. Rev. C77 (2008) 034905, [0710.5932].

[132] H. Song and U. W. Heinz, Causal viscous hydrodynamics in $2+1$ dimensions for relativistic heavy-ion collisions, Phys. Rev. C77 (2008) 064901, [0712.3715].

[133] M. Luzum and P. Romatschke, Conformal Relativistic Viscous Hydrodynamics: Applications to RHIC results at $s(N N)^{* *}(1 / 2)=200-G e V$, Phys. Rev. C78 (2008) 034915, [0804.4015].

[134] P. Benincasa, A. Buchel and A. O. Starinets, Sound waves in strongly coupled non-conformal gauge theory plasma, Nucl. Phys. B733 (2006) 160-187, [hep-th/0507026].

[135] J. Erdmenger, M. Haack, M. Kaminski and A. Yarom, Fluid dynamics of R-charged black holes, JHEP 01 (2009) 055, [0809.2488].

[136] S. Grozdanov and A. O. Starinets, On the universal identity in second order hydrodynamics, JHEP 03 (2015) 007, [1412.5685].

[137] E. Shaverin and A. Yarom, Universality of second order transport in Gauss-Bonnet gravity, JHEP 04 (2013) 013, [1211.1979].

[138] S. Grozdanov and A. O. Starinets, Zero-viscosity limit in a holographic Gauss-Bonnet liquid, Theor. Math. Phys. 182 (2015) 61-73.

[139] E. Shaverin, A breakdown of a universal hydrodynamic relation in Gauss-Bonnet gravity, 1509.05418.

[140] F. Bigazzi and A. L. Cotrone, An elementary stringy estimate of transport coefficients of large temperature QCD, JHEP 08 (2010) 128, [1006.4634].

[141] I. Kanitscheider and K. Skenderis, Universal hydrodynamics of non-conformal branes, JHEP 04 (2009) 062, [0901.1487]. 
[142] S. S. Gubser and A. Nellore, Mimicking the QCD equation of state with a dual black hole, Phys. Rev. D78 (2008) 086007, [0804.0434].

[143] C. Wu, Y. Chen and M. Huang, Fluid/gravity correspondence: Second order transport coefficients in compactified D4-branes, JHEP 01 (2017) 118, [1604.07765].

[144] G. S. Denicol, H. Niemi, E. Molnar and D. H. Rischke, Derivation of transient relativistic fluid dynamics from the Boltzmann equation, Phys. Rev. D85 (2012) 114047, [1202.4551].

[145] E. Molnár, H. Niemi, G. S. Denicol and D. H. Rischke, Relative importance of second-order terms in relativistic dissipative fluid dynamics, Phys. Rev. D89 (2014) 074010, [1308.0785].

[146] G. S. Denicol, S. Jeon and C. Gale, Transport Coefficients of Bulk Viscous Pressure in the 14-moment approximation, Phys. Rev. C90 (2014) 024912, [1403.0962].

[147] G. S. Denicol, W. Florkowski, R. Ryblewski and M. Strickland, Shear-bulk coupling in nonconformal hydrodynamics, Phys. Rev. C90 (2014) 044905, [1407.4767].

[148] A. Jaiswal, R. Ryblewski and M. Strickland, Transport coefficients for bulk viscous evolution in the relaxation time approximation, Phys. Rev. C90 (2014) 044908, [1407.7231].

[149] S. I. Finazzo, R. Rougemont, H. Marrochio and J. Noronha, Hydrodynamic transport coefficients for the non-conformal quark-gluon plasma from holography, JHEP 02 (2015) 051, [1412.2968].

[150] F. Becattini and E. Grossi, Quantum corrections to the stress-energy tensor in thermodynamic equilibrium with acceleration, Phys. Rev. D92 (2015) 045037, [1505.07760].

[151] D. Mateos, R. C. Myers and R. M. Thomson, Thermodynamics of the brane, JHEP 05 (2007) 067, [hep-th/0701132].

[152] I. Kanitscheider, K. Skenderis and M. Taylor, Precision holography for non-conformal branes, JHEP 09 (2008) 094, [0807.3324].

[153] S. Grozdanov and N. Kaplis, Constructing higher-order hydrodynamics: The third order, Phys. Rev. D93 (2016) 066012, [1507.02461].

[154] O. Saremi and K. A. Sohrabi, Causal three-point functions and nonlinear second-order hydrodynamic coefficients in AdS/CFT, JHEP 11 (2011) 147, [1105.4870].

[155] P. Arnold, D. Vaman, C. Wu and W. Xiao, Second order hydrodynamic coefficients from 3-point stress tensor correlators via AdS/CFT, JHEP 10 (2011) 033, [1105.4645].

[156] L. Girardello, M. Petrini, M. Porrati and A. Zaffaroni, Confinement and condensates without fine tuning in supergravity duals of gauge theories, JHEP 05 (1999) 026, [hep-th/9903026].

[157] L. Girardello, M. Petrini, M. Porrati and A. Zaffaroni, The Supergravity dual of $N=1$ superYang-Mills theory, Nucl. Phys. B569 (2000) 451-469, [hep-th/9909047].

[158] K. Pilch and N. P. Warner, N=2 supersymmetric RG flows and the IIB dilaton, Nucl. Phys. B594 (2001) 209-228, [hep-th/0004063]. 
[159] J. M. Maldacena and C. Nunez, Towards the large $N$ limit of pure $N=1$ superYang-Mills, Phys. Rev. Lett. 86 (2001) 588-591, [hep-th/0008001].

[160] M. Bianchi, D. Z. Freedman and K. Skenderis, How to go with an RG flow, JHEP 08 (2001) 041, [hep-th/0105276].

[161] F. Bigazzi, A. L. Cotrone, M. Petrini and A. Zaffaroni, Supergravity duals of supersymmetric four-dimensional gauge theories, Riv. Nuovo Cim. 25N12 (2002) 1-70, [hep-th/0303191].

[162] A. Buchel and J. T. Liu, Thermodynamics of the N=2* flow, JHEP 11 (2003) 031, [hep-th/0305064].

[163] R. C. Myers, Stress tensors and Casimir energies in the AdS / CFT correspondence, Phys. Rev. D60 (1999) 046002, [hep-th/9903203].

[164] S. S. Gubser, I. R. Klebanov and A. W. Peet, Entropy and temperature of black 3-branes, Phys. Rev. D54 (1996) 3915-3919, [hep-th/9602135].

[165] A. Buchel, J. T. Liu and A. O. Starinets, Coupling constant dependence of the shear viscosity in N=4 supersymmetric Yang-Mills theory, Nucl. Phys. B707 (2005) 56-68, [hep-th/0406264].

[166] P. Benincasa and A. Buchel, Transport properties of N=4 supersymmetric Yang-Mills theory at finite coupling, JHEP 01 (2006) 103, [hep-th/0510041].

[167] A. Buchel, Shear viscosity of boost invariant plasma at finite coupling, Nucl. Phys. B802 (2008) 281-306, [0801.4421].

[168] A. Buchel, Resolving disagreement for eta/s in a CFT plasma at finite coupling, Nucl. Phys. B803 (2008) 166-170, [0805.2683].

[169] R. C. Myers, M. F. Paulos and A. Sinha, Quantum corrections to eta/s, Phys. Rev. D79 (2009) 041901, [0806.2156].

[170] A. Buchel and M. Paulos, Relaxation time of a CFT plasma at finite coupling, Nucl. Phys. B805 (2008) 59-71, [0806.0788].

[171] M. Attems, J. Casalderrey-Solana, D. Mateos, I. Papadimitriou, D. Santos-Oliván, C. F. Sopuerta et al., Thermodynamics, transport and relaxation in non-conformal theories, JHEP 10 (2016) 155, [1603.01254].

[172] A. Khavaev, K. Pilch and N. P. Warner, New vacua of gauged N=8 supergravity in five-dimensions, Phys. Lett. B487 (2000) 14-21, [hep-th/9812035].

[173] H. A. Chamblin and H. S. Reall, Dynamic dilatonic domain walls, Nucl. Phys. B562 (1999) 133-157, [hep-th/9903225].

[174] S. Bhattacharyya, Constraints on the second order transport coefficients of an uncharged fluid, JHEP 07 (2012) 104, [1201.4654].

[175] K. Jensen, M. Kaminski, P. Kovtun, R. Meyer, A. Ritz and A. Yarom, Towards hydrodynamics without an entropy current, Phys. Rev. Lett. 109 (2012) 101601, [1203.3556].

[176] F. M. Haehl, R. Loganayagam and M. Rangamani, The eightfold way to dissipation, Phys. Rev. Lett. 114 (2015) 201601, [1412.1090]. 
[177] F. M. Haehl, R. Loganayagam and M. Rangamani, Adiabatic hydrodynamics: The eightfold way to dissipation, JHEP 05 (2015) 060, [1502.00636].

[178] M. A. York and G. D. Moore, Second order hydrodynamic coefficients from kinetic theory, Phys. Rev. D79 (2009) 054011, [0811.0729].

[179] M. Crossley, P. Glorioso and H. Liu, Effective field theory of dissipative fluids, 1511.03646.

[180] P. Glorioso, M. Crossley and H. Liu, Effective field theory for dissipative fluids (II): classical limit, dynamical KMS symmetry and entropy current, 1701.07817.

[181] P. Coleman, Heavy Fermions: Electrons at the Edge of Magnetism, in Handbook of Magnetism and Advanced Magnetic Materials: Fundamentals and Theory (Kronmuller and Parkin, eds.), vol. 1, pp. 95-148. John Wiley and Sons, 2007. cond-mat/0612006.

[182] P. Coleman, Heavy Fermions and the Kondo Lattice: a 21st Century Perspective, 1509.05769.

[183] P. Gegenwart, Q. Si and F. Steglich, Quantum Criticality in Heavy-fermion Metals, Nature Physics 4 (Mar., 2008) 186-197, [0712.2045].

[184] Q. Si, Quantum Criticality and Global Phase Diagram of Magnetic Heavy Fermions, 0912.0040 .

[185] Q. Si, Quantum Criticality and the Kondo Lattice, Understanding Quantum Phase Transitions. Series: Condensed Matter Physics, CRC Press (Nov., 2010) 193-216, [1012.5440].

[186] Q. Si and F. Steglich, Heavy Fermions and Quantum Phase Transitions, Science 329 (Sept., 2010) 1161-, [1102.4896].

[187] B. Keimer, S. A. Kivelson, M. R. Norman, S. Uchida and J. Zaanen, High Temperature Superconductivity in the Cuprates, 1409.4673.

[188] S. Doniach, The Kondo lattice and weak antiferromagnetism, Physica $B+C 91$ (July, 1977) 231-234.

[189] J. Kondo, Resistance Minimum in Dilute Magnetic Alloys, Prog. Theo. Phys. 32 (1964) 37-49.

[190] C. Rizzuto, Formation of Localized Moments in Metals: Experimental Bulk Properties, Rep. Prog. Phys. 37 (1974) 147.

[191] G. Grüner and A. Zawadowski, Low Temperature Properties of Kondo Alloys, in Progress in Low Temperature Physics (D. Brewer, ed.), vol. 7, Part B, pp. $591-647$. Elsevier, 1978.

[192] D. Goldhaber-Gordon, H. Shtrikman, D. Mahalu, D. Abusch-Magder, U. Meirav and M. A. Kastner, Kondo Effect in a Single-electron Transistor, Nature 391 (1998) 156-159.

[193] S. Cronenwett, T. Oosterkamp and L. Kouwenhoven, A Tunable Kondo Effect in Quantum Dots, Science 281 (1998) 540-544. 
[194] W. G. van der Wiel, S. D. Franceschi, T. Fujisawa, J. M. Elzerman, S. Tarucha and L. P. Kouwenhoven, The Kondo Effect in the Unitary Limit, Science 289 (2000) 2105-2108.

[195] K. G. Wilson, The Renormalization Group: Critical Phenomena and the Kondo Problem, Rev.Mod.Phys. 47 (1975) 773.

[196] H. R. Krishna-murthy, J. W. Wilkins and K. G. Wilson, Renormalization-group approach to the Anderson model of dilute magnetic alloys. I. Static properties for the symmetric case, Phys. Rev. B 21 (Feb, 1980) 1003-1043.

[197] H. R. Krishna-murthy, J. W. Wilkins and K. G. Wilson, Renormalization-group approach to the Anderson model of dilute magnetic alloys. II. Static properties for the asymmetric case, Phys. Rev. B 21 (Feb, 1980) 1044-1083.

[198] N. Andrei, Diagonalization of the Kondo Hamiltonian, Phys. Rev. Lett. 45 (Aug, 1980) 379-382.

[199] P. Wiegmann, Exact Solution of s-d Exchange Model at T=0, Sov. Phys. JETP Lett. 31 (1980) 364.

[200] N. Andrei, K. Furuya and J. H. Lowenstein, Solution of the Kondo problem, Rev. Mod. Phys. 55 (Apr, 1983) 331-402.

[201] A. Tsvelick and P. Wiegmann, Exact Results in the Theory of Magnetic Alloys, Advances in Physics 32 (1983) 453-713.

[202] P. Coleman and N. Andrei, Diagonalisation of the Generalised Anderson Model, Jour. Phys. C19 (1986) 3211-3233.

[203] N. Andrei, Integrable Models in Condensed Matter Physics, cond-mat/9408101.

[204] P. Zinn-Justin and N. Andrei, The Generalized Multi-channel Kondo Model: Thermodynamics and Fusion Equations, Nucl. Phys. B528 (1998) 648-682, [cond-mat/9801158].

[205] A. Jerez, N. Andrei and G. Zaránd, Solution of the Multichannel Coqblin-Schrieffer Impurity Model and Application to Multilevel Systems, Phys. Rev. B58 (1998) 3814-3841, [cond-mat/9803137].

[206] P. Coleman, Mixed Valence as an Almost Broken Symmetry, Phys. Rev. B35 (Apr, 1987) 5072-5116.

[207] N. Bickers, Review of Techniques in the Large-N Expansion for Dilute Magnetic Alloys, Rev. Mod. Phys. 59 (Oct, 1987) 845-939.

[208] O. Parcollet and A. Georges, Transition from Overscreening to Underscreening in the Multichannel Kondo Model: Exact Solution at Large N, Phys. Rev. Lett. 79 (1997) 4665-4668, [cond-mat/9707337].

[209] O. Parcollet, A. Georges, G. Kotliar and A. Sengupta, Overscreened Multi-channel $S U(N)$ Kondo Model: Large-N Solution and Conformal Field Theory, Phys. Rev. B58 (Aug., 1998) 3794-3813, [cond-mat/9711192].

[210] I. Affleck, A current algebra approach to the Kondo effect, Nucl. Phys. B336 (1990) 517. 
[211] I. Affleck and A. Ludwig, The Kondo Effect, Conformal Field Theory and Fusion Rules, Nucl.Phys. B352 (1991) 849-862.

[212] I. Affleck and A. Ludwig, Critical Theory of Overscreened Kondo Fixed Points, Nucl.Phys. B360 (1991) 641-696.

[213] I. Affleck and A. Ludwig, Universal Non-integer 'Ground State Degeneracy' in Critical Quantum Systems, Phys.Rev.Lett. 67 (1991) 161-164.

[214] I. Affleck and A. Ludwig, Exact Conformal-field-theory Results on the Multichannel Kondo Effect: Single-fermion Green's function, Self-energy, and Resistivity, Phys.Rev. B48 (1993) 7297-7321.

[215] I. Affleck, Conformal Field Theory Approach to the Kondo Effect, Acta Phys. Polon. B26 (1995) 1869-1932, [cond-mat/9512099].

[216] A. Hewson, The Kondo Model to Heavy Fermions. Cambridge University Press, 1993.

[217] D. L. Cox and A. Zawadowski, Exotic Kondo Effects in Metals: Magnetic Ions in a Crystalline Electric Field and Tunnelling Centres, Advances in Physics 47 (1998) 599-942, [cond-mat/9704103].

[218] I. Affleck, The Kondo screening cloud: what it is and how to observe it, 0911.2209.

[219] C. Jayaprakash, H. Krishna-murthy and J. Wilkins, Two-Impurity Kondo Problem, Phys. Rev. Lett. 47 (Sep, 1981) 737-740.

[220] B. Jones and C. Varma, Study of Two Magnetic Impurities in a Fermi Gas, Phys. Rev. Lett. 58 (Mar, 1987) 843-846.

[221] B. Jones, C. Varma and J. Wilkins, Low-Temperature Properties of the Two-Impurity Kondo Hamiltonian, Phys. Rev. Lett. 61 (Jul, 1988) 125-128.

[222] B. Jones, Antiferromagnetic Phase Instability in the Two-Impurity Kondo Problem, in Field Theories in Condensed Matter Physics: A Workshop (Z. Tesanovic, ed.), pp. 87-103. Addison-Wesley, 1990.

[223] R. M. Fye, J. E. Hirsch and D. J. Scalapino, Kondo effect versus indirect exchange in the two-impurity Anderson model: A Monte Carlo study, Phys. Rev. B 35 (Apr, 1987) 4901-4908.

[224] B. A. Jones, B. G. Kotliar and A. J. Millis, Mean-field analysis of two antiferromagnetically coupled Anderson impurities, Phys. Rev. B 39 (Feb, 1989) $3415-3418$

[225] A. Millis, B. Kotliar and B. Jones, The Two Kondo Impurity Problem: A Large N Biased Review, in Field Theories in Condensed Matter Physics: A Workshop (Z. Tesanovic, ed.), pp. 159-166. Addison-Wesley, 1990.

[226] R. M. Fye and J. E. Hirsch, Quantum Monte Carlo study of the two-impurity Kondo Hamiltonian, Phys. Rev. B 40 (Sep, 1989) 4780-4796.

[227] B. Jones and C. Varma, Critical Point in the Solution of the Two Magnetic Impurity Problem, Phys. Rev. B40 (Jul, 1989) 324-329.

[228] I. Affleck and A. W. W. Ludwig, Exact critical theory of the two impurity Kondo model, Phys. Rev. Lett. 68 (1992) 1046-1049. 
[229] I. Affleck, A. Ludwig and B. Jones, Conformal-field-theory Approach to the Two-impurity Kondo Problem: Comparison with Numerical Renormalization-group Results, Phys. Rev. B52 (Oct., 1995) 9528-9546, [cond-mat/9409100].

[230] J. Gan, Mapping the Critical Point of the Two-Impurity Kondo Model to a Two-Channel Problem, Phys. Rev. Lett. 74 (Mar, 1995) 2583-2586.

[231] J. Gan, Solution of the two-impurity Kondo model: Critical point, Fermi-liquid phase, and crossover, Phys. Rev. B 51 (Apr, 1995) 8287-8309.

[232] A. Georges and A. M. Sengupta, Solution of the Two-Impurity, Two-Channel Kondo Model, Phys. Rev. Lett. 74 (Apr, 1995) 2808-2811.

[233] R. M. Fye, "Anomalous fixed point behavior" of two Kondo impurities: A reexamination, Phys. Rev. Lett. 72 (Feb, 1994) 916-919.

[234] K. Ingersent and B. A. Jones, Low-temperature physics of the two-impurity, two-channel Kondo model, Physica B: Condensed Matter 199 (1994) 402 - 405.

[235] J. B. Silva, W. L. C. Lima, W. C. Oliveira, J. L. N. Mello, L. N. Oliveira and J. W. Wilkins, Particle-Hole Asymmetry in the Two-Impurity Kondo Model, Phys. Rev. Lett. 76 (Jan, 1996) 275-278.

[236] B. Jones, The Kondo Effect, in Handbook of Magnetism and Advanced Magnetic Materials: Fundamentals and Theory (Kronmuller and Parkin, eds.), vol. 1, pp. 149-163. John Wiley and Sons, 2007.

[237] D.-H. Lee and J. Toner, Kondo effect in a Luttinger liquid, Phys. Rev. Lett. 69 (Dec, 1992) 3378-3381.

[238] A. Furusaki and N. Nagaosa, Kondo effect in a Tomonaga-Luttinger liquid, Phys. Rev. Lett. 72 (Feb, 1994) 892-895.

[239] P. Fröjdh and H. Johannesson, Kondo Effect in a Luttinger Liquid: Exact Results from Conformal Field Theory, Phys. Rev. Lett. 75 (Jul, 1995) 300-303.

[240] P. Fröjdh and H. Johannesson, Magnetic impurity in a Luttinger liquid: A conformal field theory approach, Phys. Rev. B 53 (Feb, 1996) 3211-3236.

[241] A. Furusaki, Kondo Problems in Tomonaga-Luttinger Liquids, Journal of the Physical Society of Japan 74 (Jan., 2005) 73-79, [cond-mat/0409016].

[242] P. Fulde, V. Zevin and G. Zwicknagl, Model for heavy-fermion behavior of Nd1.8Ce0.2CuO4, Zeitschrift für Physik B Condensed Matter 92 (1993) 133-135.

[243] T. Schork and P. Fulde, Interaction of a magnetic impurity with strongly correlated conduction electrons, Phys. Rev. B 50 (Jul, 1994) 1345-1350.

[244] S. Kachru, A. Karch and S. Yaida, Holographic Lattices, Dimers, and Glasses, Phys.Rev. D81 (2010) 026007, [0909.2639].

[245] S. Sachdev, Holographic Metals and the Fractionalized Fermi Liquid, Phys.Rev.Lett. 105 (2010) 151602, [1006.3794].

[246] S. Kachru, A. Karch and S. Yaida, Adventures in Holographic Dimer Models, New J.Phys. 13 (2011) 035004, [1009.3268]. 
[247] S. Sachdev, Strange Metals and the AdS/CFT Correspondence, J.Stat.Mech. 1011 (2010) P11022, [1010.0682].

[248] W. Mück, The Polyakov Loop of Anti-symmetric Representations as a Quantum Impurity Model, Phys.Rev. D83 (2011) 066006, [1012.1973].

[249] A. Faraggi and L. Pando Zayas, The Spectrum of Excitations of Holographic Wilson Loops, JHEP 1105 (2011) 018, [1101.5145].

[250] K. Jensen, S. Kachru, A. Karch, J. Polchinski and E. Silverstein, Towards a Holographic Marginal Fermi Liquid, Phys.Rev. D84 (2011) 126002, [1105.1772].

[251] N. Karaiskos, K. Sfetsos and E. Tsatis, Brane Embeddings in Sphere Submanifolds, Class.Quant.Grav. 29 (2012) 025011, [1106.1200].

[252] S. Harrison, S. Kachru and G. Torroba, A Maximally Supersymmetric Kondo Model, Class.Quant.Grav. 29 (2012) 194005, [1110.5325].

[253] P. Benincasa and A. Ramallo, Fermionic Impurities in Chern-Simons-Matter Theories, JHEP 1202 (2012) 076, [1112.4669].

[254] A. Faraggi, W. Mück and L. Pando Zayas, One-loop Effective Action of the Holographic Antisymmetric Wilson Loop, Phys.Rev. D85 (2012) 106015, [1112.5028].

[255] P. Benincasa and A. Ramallo, Holographic Kondo Model in Various Dimensions, JHEP 1206 (2012) 133, [1204.6290].

[256] H. Matsueda, Multiscale Entanglement Renormalization Ansatz for Kondo Problem, 1208.2872.

[257] G. Itsios, K. Sfetsos and D. Zoakos, Fermionic Impurities in the Unquenched ABJM, JHEP 1301 (2013) 038, [1209.6617].

[258] J. Camino, A. Paredes and A. Ramallo, Stable Wrapped Branes, JHEP 05 (2001) 011, [hep-th/0104082].

[259] S. Yamaguchi, Wilson Loops of Anti-symmetric Representation and D5-branes, JHEP 0605 (2006) 037, [hep-th/0603208].

[260] J. Gomis and F. Passerini, Holographic Wilson Loops, JHEP 08 (2006) 074, [hep-th/0604007].

[261] J. Gomis and F. Passerini, Wilson Loops as D3-Branes, JHEP 0701 (2007) 097, [hep-th/0612022].

[262] M. Blake, A. Donos and D. Tong, Holographic Charge Oscillations, JHEP 04 (2015) 019, [1412.2003].

[263] G. T. Horowitz, J. E. Santos and D. Tong, Optical Conductivity with Holographic Lattices, JHEP 07 (2012) 168, [1204.0519].

[264] D. Bensimon, A. Jerez and M. Lavagna, Intermediate Coupling Fixed Point Study in the Overscreened Regime of Generalized Multichannel SU(N) Kondo Models, Phys. Rev. B73 (2006) 224445.

[265] P. Nozières and A. Blandin, Kondo Effect in Real Metals, J. Phys. France 41 (1980) 193-211. 
[266] T. Senthil, S. Sachdev and M. Vojta, Fractionalized Fermi Liquids, Phys. Rev. Lett. 90 (May, 2003) 216403, [cond-mat/0209144].

[267] T. Senthil, M. Vojta and S. Sachdev, Weak Magnetism and Non-Fermi liquids Near Heavy-fermion Critical Points, Phys. Rev. B69 (Jan., 2004) 035111, [cond-mat/0305193].

[268] A. Auerbach and D. P. Arovas, Schwinger Bosons Approaches to Quantum Antiferromagnetism, 0809.4836.

[269] M. Mathur, I. Raychowdhury and R. Anishetty, SU(N) irreducible Schwinger bosons, Journal of Mathematical Physics 51 (Sept., 2010) 093504, [1003.5487].

[270] P. Kraus, Lectures on Black Holes and the AdS(3)/CFT(2) Correspondence, Lect. Notes Phys. 755 (2008) 193-247, [hep-th/0609074].

[271] M. Bianchi, D. Z. Freedman and K. Skenderis, Holographic renormalization, Nucl. Phys. B631 (2002) 159-194, [hep-th/0112119].

[272] I. Papadimitriou and K. Skenderis, Thermodynamics of asymptotically locally AdS spacetimes, JHEP 08 (2005) 004, [hep-th/0505190].

[273] I. Papadimitriou, Holographic renormalization as a canonical transformation, JHEP 11 (2010) 014, [1007.4592].

[274] A. Castro, D. Grumiller, F. Larsen and R. McNees, Holographic Description of AdS(2) Black Holes, JHEP 0811 (2008) 052, [0809. 4264].

[275] M. Fujita, S. Harrison, A. Karch, R. Meyer and N. M. Paquette, Towards a Holographic Bose-Hubbard Model, JHEP 04 (2015) 068, [1411.7899].

[276] D. Marolf and S. F. Ross, Boundary Conditions and New Dualities: Vector Fields in AdS/CFT, JHEP 11 (2006) 085, [hep-th/0606113].

[277] C. Fefferman and C. R. Graham, Conformal Invariants, in Elie Cartan et les Mathematiques d'aujourd'hui, p. 95. Asterique, 1985.

[278] D. Martelli and W. Mueck, Holographic renormalization and Ward identities with the Hamilton-Jacobi method, Nucl. Phys. B654 (2003) 248-276, [hep-th/0205061].

[279] W. Chemissany and I. Papadimitriou, Lifshitz holography: The whole shebang, JHEP 01 (2015) 052, [1408.0795].

[280] I. Papadimitriou and K. Skenderis, Correlation functions in holographic RG flows, JHEP 10 (2004) 075, [hep-th/0407071].

[281] B. C. van Rees, Holographic renormalization for irrelevant operators and multi-trace counterterms, JHEP 08 (2011) 093, [1102.2239].

[282] B. C. van Rees, Irrelevant deformations and the holographic Callan-Symanzik equation, JHEP 10 (2011) 067, [1105.5396].

[283] I. Papadimitriou, Multi-Trace Deformations in AdS/CFT: Exploring the Vacuum Structure of the Deformed CFT, JHEP 0705 (2007) 075, [hep-th/0703152].

[284] E. Witten, Multitrace operators, boundary conditions, and AdS / CFT correspondence, hep-th/0112258. 
[285] C. Imbimbo, A. Schwimmer, S. Theisen and S. Yankielowicz, Diffeomorphisms and holographic anomalies, Class. Quant. Grav. 17 (2000) 1129-1138, [hep-th/9910267].

[286] A. Schwimmer and S. Theisen, Diffeomorphisms, anomalies and the Fefferman-Graham ambiguity, JHEP 08 (2000) 032, [hep-th/0008082].

[287] A. Barut and R. Raczka, Theory of Group Representations and Applications. World Scientific Publishing, Singapore, second ed., 1986.

[288] G. T. Horowitz and M. M. Roberts, Zero Temperature Limit of Holographic Superconductors, JHEP 11 (2009) 015, [0908.3677].

[289] J. Erdmenger, M. Flory and M.-N. Newrzella, Bending branes for DCFT in two dimensions, JHEP 01 (2015) 058, [1410.7811].

[290] P. Coleman, Introduction to Many-Body Physics. Cambridge University Press, 2015.

[291] S. Sachdev and J.-W. Ye, Gapless spin fluid ground state in a random, quantum Heisenberg magnet, Phys. Rev. Lett. 70 (1993) 3339, [cond-mat/9212030].

[292] A. Kitaev, A Simple Model of Quantum Holography, talks at the KITP Strings seminar and Entanglement 2015 program (Feb. 12, Apr. 7, and May 27, 2015) .

[293] J. Polchinski and V. Rosenhaus, The Spectrum in the Sachdev-Ye-Kitaev Model, JHEP 04 (2016) 001, [1601.06768].

[294] J. Maldacena and D. Stanford, Remarks on the Sachdev-Ye-Kitaev model, Phys. Rev. D94 (2016) 106002, [1604.07818].

[295] O. Aharony and D. Kutasov, Holographic Duals of Long Open Strings, Phys. Rev. D78 (2008) 026005, [0803.3547].

[296] R. Flint, M. Dzero and P. Coleman, Heavy electrons and the symplectic symmetry of spin, Nature Physics 4 (Aug., 2008) 643, [0710.1126].

[297] R. Flint, M. Dzero and P. Coleman, Supplementary material to Heavy electrons and the symplectic symmetry of spin, 0710.1128.

[298] A. Cherman and A. Nellore, Universal relations of transport coefficients from holography, Phys. Rev. D80 (2009) 066006, [0905.2969]. 